\title{
Electrical characterization of Metal - Amorphous Semiconductor - Semiconductor diodes - a general conduction model
}

\author{
Dissertation \\ zur Erlangung des mathematisch-naturwissenschaftlichen Doktorgrades \\ "Doktor rerum naturalium" \\ der Georg-August-Universität Göttingen \\ im Promotionsprogramm Prophys \\ der Georg-August-Universität Göttingen
}

vorgelegt von

Marc Brötzmann

aus Braunschweig

Göttingen, 2012 
Betreuungsausschuss

Prof. Dr. Hans Hofsäss, II. Physikalisches Institut, Universität Göttingen

Dr. Ulrich Vetter, II. Physikalisches Institut/ AG Prof. Hofsäss, Universität Göttingen

\section{Mitglieder der Prüfungskommission}

Referent: Prof. Dr. Hans Hofsäss, II. Physikalisches Institut, Universität Göttingen

Korreferent: Prof. Dr. Michael Seibt, IV. Physikalisches Institut, Universität Göttingen

Weitere Mitglieder der Prüfungskommission:

Prof. Dr. Cynthia A. Volkert, Materialphysikalisches Institut, Universität Göttingen

Prof. Dr. Angela Rizzi, IV. Physikalisches Institut, Universität Göttingen

PD Dr. Jörn Grosse-Knetter, II. Physikalisches Institut/ AG Prof. Quadt, Universität Göttingen

Prof. Dr. Hans-Ulrich Krebs, Materialphysikalisches Institut, Universität Göttingen

Tag der mündlichen Prüfung: 28.01.2013 


\section{Contents}

1 Introduction 1

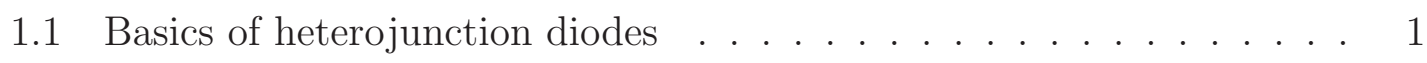

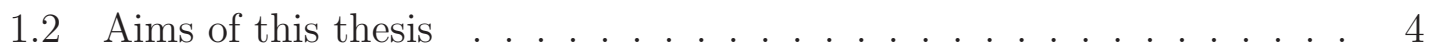

2 Fundamentals of MASS diode structures 5

2.1 Introduction . . . . . . . . . . . . . . . . 5

2.2 Semiconducting Substrates . . . . . . . . . . . . 6

2.2 .1 Silicon . . . . . . . . . . . . . . . 6

2.2 .2 n-type $6 \mathrm{H}-\mathrm{SiC} \ldots \ldots \ldots \ldots \ldots$

2.2 .3 Zinc-oxide $(\mathrm{ZnO}) \ldots \ldots \ldots \ldots$

2.3 Ohmic back contact preparation . . . . . . . . . . . 12

2.3.1 Si based MASS diodes . . . . . . . . . . . . . . 12

2.3.2 SiC based MASS diodes . . . . . . . . . . . . . . . 14

2.3.3 ZnO based MASS diodes . . . . . . . . . . . . . . . . 16

2.4 Amorphous Semiconductors . . . . . . . . . . . . . . 17

2.4 .1 tetrahedral amorphous carbon $($ ta-C) . . . . . . . . 17

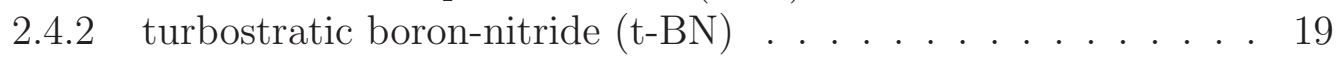

2.5 Thin-film growth - Mass Selected Ion-Beam Deposition (MSIBD) . . 21

2.5 .1 Ion source . . . . . . . . . . . . . . . . 21

2.5.2 The ion-accelerator ADONIS . . . . . . . . . . 23

2.5.3 Deposition of ta-C and t-BN thin films ......... . . 24

2.6 Metal top contacts . . . . . . . . . . . . . 25

3 Electrical properties of MASS diodes 27

3.1 Ohmic conduction . . . . . . . . . . . . . . . 27

3.2 Hopping mechanisms . . . . . . . . . . . . . . . . . . . 29

3.2.1 Variable Range Hopping . . . . . . . . . . . . . . . . . . . . 30

3.3 Frenkel-Poole effect . . . . . . . . . . . . . . . . . . 31

3.4 Schottky effect . . . . . . . . . . . . . . . . 32

3.5 p-n junction diode - the Shockley model . . . . . . . . . . . . . . 34

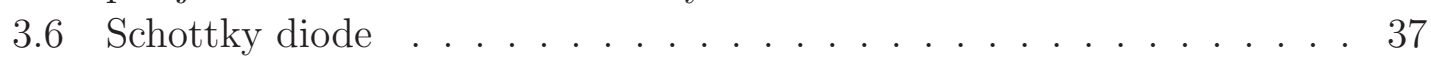

3.7 Tunneling mechanisms . . . . . . . . . . . . . . . . . 39

3.8 Photoconductivity in semiconductors . . . . . . . . . . . . . 40 
4 Characterization techniques 43

4.1 Structural characterization . . . . . . . . . . . . . . . . 43

4.1.1 X-ray photoemission spectroscopy (XPS) . . . . . . . . 43

4.1.2 Transmission-Electron-Microscopy (TEM) . . . . . . . . . 45

4.2 Electrical characterization $(\mathrm{DC}) \ldots \ldots \ldots \ldots \ldots$

4.2 .1 Probe geometry . . . . . . . . . . . . . 47

4.2 .2 Mounting of samples . . . . . . . . . . . . . . 49

4.2 .3 Low-temperature setup . . . . . . . . . . . . . . . . . 50

4.2 .4 High-temperature setup . . . . . . . . . . . . . . . 52

4.2 .5 Data recording and processing . . . . . . . . . . . . 53

4.3 Impedance spectroscopy AC . . . . . . . . . . . . . 55

4.4 Photoresponse measurements . . . . . . . . . . . . 57

4.4 .1 Case study experiments . . . . . . . . . . . . . . 57

4.4.2 Time-resolved photoresponse . . . . . . . . . . . . . . 59

5 Structural characterization 63

5.1 Introduction . . . . . . . . . . . . . . . . . 63

5.2 XPS-measurements . . . . . . . . . . . . . . . . 63

5.2 .1 ta-C samples . . . . . . . . . . . . . . . . 65

5.2 .2 t-BN samples $\ldots \ldots \ldots \ldots \ldots \ldots$

5.2 .3 Contaminant analysis . . . . . . . . . . . . . . . . . 69

5.3 HRTEM-measurements . . . . . . . . . . . . . . . . . . . . . 71

$5.3 .1 \mathrm{t}-\mathrm{BN} / \mathrm{ZnO}$ heterostructures $\ldots \ldots \ldots \ldots . \ldots . \ldots 75$

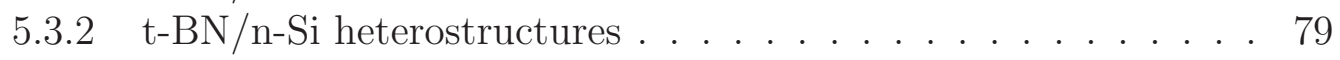

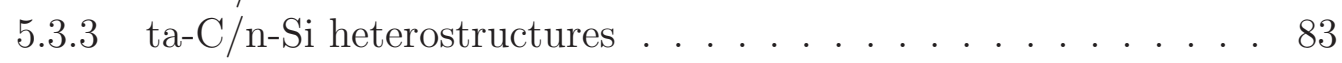

$5.3 .4 \quad \mathrm{t}-\mathrm{BN} / \mathrm{p}-\mathrm{Si}$ heterostructures . . . . . . . . . . . . . . 86

5.3 .5 ta-C $/ \mathrm{p}-\mathrm{Si}$ heterostructures $\ldots \ldots \ldots \ldots$

$5.3 .6 \quad \mathrm{t}-\mathrm{BN} / 6 \mathrm{H}-\mathrm{SiC}$ heterostructures $\ldots \ldots \ldots \ldots . . \ldots \ldots 7$

5.3 .7 ta-C $/ 6 \mathrm{H}-\mathrm{SiC}$ heterostructures $\ldots \ldots \ldots \ldots . . \ldots \ldots 101$

5.3 .8 Surface properties of $6 \mathrm{H}-\mathrm{SiC} \ldots \ldots \ldots \ldots \ldots$

5.4 Summary . . . . . . . . . . . . . . . . . . . . 111

6 Electrical characterization $\quad 115$

$6.1 \mathrm{I}-\mathrm{V}$ characteristics of reference samples . . . . . . . . . . . 116

6.1.1 Metal/t-BN/metallic Si heterostuctures . . . . . . . . . 118

6.1.2 Metal/ta-C/metallic Si heterostuctures . . . . . . . . . 123

6.1 .3 Contaminant analysis . . . . . . . . . . . . . . 127

$6.2 \mathrm{t}$-BN/ZnO heterostructures $\ldots \ldots \ldots \ldots$

$6.3 \mathrm{ta}-\mathrm{C} / \mathrm{p}-\mathrm{Si}$ heterostructures . . . . . . . . . . . . . . . . . . 140

6.3.1 Comparison of different top-contact materials . . . . . . 149

6.3.2 Comparison with ta-C/n-Si heterostructures . . . . . . 150

6.4 SiC-based MASS diodes . . . . . . . . . . . . . . . . 153

$6.4 .1 \mathrm{t}-\mathrm{BN} / \mathrm{SiC}$ heterostructures $\ldots \ldots \ldots \ldots \ldots$

6.4 .2 ta-C/SiC heterostructures $\ldots \ldots \ldots \ldots$ 
6.4.3 The effect of $\mathrm{Cu}$ contaminants . . . . . . . . . . . . . . . . . . . . . . . . . .

6.5 Additional MASS heterostructures . . . . . . . . . . . . . . 168

6.5.1 t-BN/p-Si heterostructures . . . . . . . . . . . . . . 168

6.5.2 ta-C/ZnO heterostructures . . . . . . . . . . . . . . . . . . . . . . . . . . . . . . . . . .

6.6 Summary . . . . . . . . . . . . . . . . . . . . . . . 172

7 Photoresponse of MASS diodes $\quad \mathbf{1 7 7}$

7.1 Case studies of photoconductivity measurements . . . . . . . . . 177

7.1.1 Reference samples . . . . . . . . . . . . . . . 177

7.1.2 MASS heterojunctions . . . . . . . . . . . . . . . 180

7.2 Time-resolved photoresponse measurements . . . . . . . . . . . . 183

7.2.1 Au/Cr/ta-C/p-Si heterostructures . . . . . . . . . . . . . . . 184

$7.2 .2 \mathrm{Au} / \mathrm{Cr} / \mathrm{t}-\mathrm{BN} / \mathrm{SiC}$ heterostructures . . . . . . . . . . . . . . . 186

$7.2 .3 \mathrm{Au} / \mathrm{Cr} / \mathrm{ta}-\mathrm{C} / \mathrm{SiC}$ heterostructures . . . . . . . . . . . . . . . 188

$7.2 .4 \mathrm{Au} / \mathrm{Cr} / \mathrm{t}-\mathrm{BN} / \mathrm{ZnO}$ heterostructures . . . . . . . . . . . . . . . 190

7.3 Summary . . . . . . . . . . . . . . . . . . . . . . . . . . . 192

8 Impedance spectroscopy $\quad \mathbf{1 9 3}$

8.1 Fundamentals . . . . . . . . . . . . . . . . . . . . . . . . . . . . 193

8.1.1 Complex capacitance and constant phase element (CPE) . . 193

8.1 .2 Capacitances of diodes . . . . . . . . . . . . . . 196

8.1.3 Parameter determination and numerical simulations . . . . . 196

8.2 AC properties of thin-film reference samples . . . . . . . . . . . . . 198

8.2.1 Metal/ta-C/metallic Si heterostuctures . . . . . . . . . . . . 198

8.2.2 Metal/t-BN/metallic Si heterostuctures . . . . . . . . . . . 201

8.3 AC properties of MASS heterojunctions . . . . . . . . . . . . 204

8.3 .1 ta-C/p-Si heterostructures . . . . . . . . . . . 205

8.3.2 ta-C/SiC heterostructures . . . . . . . . . . . . . 208

$8.3 .3 \mathrm{t}-\mathrm{BN} / \mathrm{SiC}$ heterostructures . . . . . . . . . . . . . . . . . . . . . . . . . . . . . . . . . . . .

8.4 Summary . . . . . . . . . . . . . . . . . . . . . . . . . . 214

9 Conclusion and Outlook 215

List of figures

\begin{tabular}{ll} 
Bibliography & 227 \\
\hline
\end{tabular} 



\section{Chapter 1}

\section{Introduction}

\subsection{Basics of heterojunction diodes}

One of the most common configurations of semiconductor based devices is the rectifying p-n junction diode which was first developed by Shockley in 1949 [1].

The corresponding development of the transistor by Bardeen and Brittain in 1947 (bipolar transistor) as well as by Shockley in 1949 (on a p-n junction based system) [1 3] led to the development of integrated circuits by Kilby in 1958 [4] and therefore provided a key element in the development of modern electronics based on semiconductor technology [5].

In the past decades, the resulting progress in semiconductor technology associated with increasing knowledge of semiconductor materials and fabrication processes resulted in the miniaturization of integrated circuit (IC) structures. In addition, a variety of semiconductor-based electronic components [5] such as different basic types of transistor and integrated circuits as well as diode based devices such as photo-diodes, light-emitting diodes (LEDs) [6 8] or solar cells, originally developed by Chapin et al. in 1954 using a silicon p-n junction [9], were developed [5].

In the course of progressing miniaturization processes and the incorporation of wideband-gap semiconductors in industrial-grade devices such as $\mathrm{SiC}, \mathrm{GaN}$ and several other wide band-gap materials [10], the optimization of fabrication processes as well as the basic understanding of the electrical properties of fabricated devices became increasingly important, including studies about possible interference effects due to imperfections of the related structural properties.

While the dimensions of the device structures are related to the accuracy of the underlying preparation steps, especially the precision of the frequently used lithography techniques, the corresponding electrical properties of fabricated devices are often affected by interface states inside the device structure as well as defect states originating from the micro-structural properties and the composition of the device material [5].

Therefore, aside from the continuous improvements in manufacturing techniques and device layout, the basic analysis of effects related to unintentionally arising 
imperfections in structural properties and the corresponding deviations from ideal electrical behavior becomes also more important.

In this context, most of up-to-date IC device applications start to suffer from increased leakage currents due to the miniaturization processes as the progressive reduction of insulator layer thicknesses lead to arising tunneling currents [11]. In addition, the electrical properties of rectifying devices such as breakdown stability at high electric fields and high temperatures are affected by unintentional recombination processes inside the corresponding depletion regions. These recombination processes primarily emerge due to the presence of defect centers inside the materials as well as arising interface states inside typically layered heterostructures. The effects of structural properties on the resulting electrical properties of related device structures can frequently be observed in pre-commercial grade materials and were, for instance, described in great detail for the research on silicon carbide ( $\mathrm{SiC}$ ) substrates and corresponding device applications [12 27].

Focusing on a variety of different diode structures such as p-n junction, Schottky and heterojunction diodes, photo diode and solar cell applications, the electrical properties of most commercially fabricated diode structures can be described by the theory of Sah, Noyce and Shockley, published in 1957 [28] and was originally developed for the characteristics of $\mathrm{p}-\mathrm{n}$ diode structures, as well as appropriate extensions of the other diode compositions, e.g. referring to Schottky diodes [29 31].

The corresponding current-voltage characteristic of a p-n junction diode can be described by the Shockley equation [32, 33]:

$$
I=I_{s} \cdot\left(\exp \left[\frac{q V}{n k T}\right]-1\right)
$$

where $I_{s}$ is the saturation current, $T$ the temperature and $n$ the so called ideality factor of the diode which is directly linked to the recombination processes inside the diode structure.

For ideal p-n diodes, in particular, desirable for solar cell application, an ideality factor approaching unity is desired. The ideality factors of real p-n diodes are also described by the Sah-Noyce-Shockley theory yielding values of $n=1$ at a low voltage and $n=2$ at higher voltages. If there were no defects present, the total diode current would be based exclusively on diffusion and $n$ would be 1 . Therefore, no recombination processes are expected inside the space-charge region. The resulting minimization of leakage current loss at reverse bias and increased rectification characteristic are favorable properties of such devices.

As recombination processes are driven by defects, more defects lead to more spacecharge recombination increasing $n$ up to 2 (the "non-ideal" case). For p-n diodes, an ideality factor $n>2$ is not covered by the Sah-Noyce-Shockley theory and suggests the presence of surface or interface states, indicating that the junction is far from being ideal [34].

Nevertheless, several studies report on a variety of homo- and heterojunction diodes as well as of metal-insulator-semiconductor (MIS) structures exhibiting large ideality factors ranging from $n=2$ to $n \approx 22$ [8, 34 41]. In addition, other publications 
report on large turn-on voltage (or threshold voltage), in some cases in excess of $10 \mathrm{~V}$, extracted from I-V curves of heterojunction diodes, which may also be an indication of a large ideality factor 42 44].

In this context, Breitenstein et al. [37] introduced a model to describe ideality factors $n>2$ arising in silicon solar cell devices based on coupled defects [45] and donor acceptor pair recombination, both leading to an increased recombination current. It is stated that for a high density of defect states, hopping conduction in the defect volume may govern the reverse conductivity of the devices. However, the model is only able to explain ideality factors in the range of $n<6$.

Furthermore, several groups analyzed the phenomenon on the basis of MIS-structures [46, 47], and basically linked the effect of increased ideality factors to arising interface states originating from metal/insulator and insulator/semiconductor interface properties inside the particular MIS-diode structures [30, 41, 48 52].

All these approaches 1 to explain high ideality factors are sound and useful as long as a well defined junction structure exists. In particular, the temperature dependence is a crucial test of the different models. However, ideality factors $n>6$ are not covered by these approaches and none of them provided a generally valid conduction model for more than one specific heterojunction system.

In addition, similar heterojunction diodes on the basis of metal/diamond like amorphous carbon (DLC)/silicon heterostructures were investigated by several groups 54 56]. Instead of an insulating layer, these heterojunctions are equipped with an amorphous diamond-like carbon thin-film deposited onto the Si-substrates by filtered cathodic vacuum arc (FCVA) deposition [54] as well as rf-plasma assisted chemical vapor deposition (CVD) methods using methane [55, 56]. All of these heterojunctions exhibited pronounced rectifying current-voltage (I-V) characteristics but also relatively high turn-on voltages.

The rectifying character of these kind of heterojunctions can also be verified based on publications of the author's work-group by Ronning et al. [57] as well as Hofsäss et al. [58]. The corresponding heterostructures were based on hydrogen-free tetrahedral amorphous carbon (ta-C) thin-films, the most $\mathrm{sp}^{3}$-rich form of diamond-like carbon, grown by mass-selected ion-beam deposition (MSIBD).

In this context, the dominating conduction mechanism of the grown thin-films around room temperature was found to be governed by thermal activation of electrons trapped in localized bound states, e.g. Frenkel-Poole conduction [32, 59], due to the amorphous character of the ta-C thin-films.

The same conduction mechanism (Frenkel-Poole) was also reported for defect-rich turbostratic boron-nitride thin-films by Ronning et al. [60] as well as by Nose et al., who also analyzed the structural properties of the grown thin-films using transmission-electron microscopy (TEM) measurements [61 63].

However, despite a general idea of the dominating conduction mechanism inside the grown thin-films as well as basic information about the structural properties of the grown thin-film, there is still no general conduction model available for such kind of

\footnotetext{
${ }^{1}$ described in more detail in reference 53 .
} 
heterostructures. In addition, temperature dependencies of the heterojunction characteristics as well as substantial explanations regarding the origin of the rectifying characteristics and considerations of possibly present interface structures have also not been taken into account.

\subsection{Aims of this thesis}

Therefore, the aims of this thesis are the comprehensive analysis of dominating conduction mechanism inside amorphous thin-films as well as the effect of different amorphous structures on the electrical properties of several heterojunction systems in terms of defect and interface configuration. Both will be performed on the basis of detailed structural characterizations of the corresponding heterostructures.

For that reason, various heterostructures on the basis of different single-crystalline semiconducting substrates such as silicon $(\mathrm{Si})$, silicon carbide in $6 \mathrm{H}$ configuration $(6 \mathrm{H}-\mathrm{SiC})$ and zinc oxide $(\mathrm{ZnO})$ covered with a thin-film of an amorphous or highly disordered material such as tetrahedral amorphous carbon (ta-C) and so called turbostratic boron nitride (t-BN) as well as an evaporated metal contact on top have been investigated during this work. The thin-films were grown by mass-selected ion-beam deposition (MSIBD) including reference samples based on highly doped n-type silicon substrates in order to study the corresponding conduction mechanisms of the thin-films. As a side-effect, additional ion-induced defect structures can also be investigated similar to doping of semiconductors by ion-beam implantation [5]. The fundamentals of these structures including material properties as well as sample preparation steps are described in detail in chapter 2 .

Furthermore, the structural properties of each metal-amorphous semiconductorsemiconductor (MASS) heterostructure (see section 2.1) has been investigated using cross-sectional high resolution transmission electron microscopy measurements (HRTEM) as shown in chapter 5 .

Based on these results as well as the theory of corresponding conduction mechanisms, summarized in chapter 3, the electrical properties of all grown heterostructures were investigated using temperature dependent current-voltage (I-V) measurements resulting in the development of a general, temperature dependent conduction model for all metal-amorphous semiconductor-semiconductor (MASS) heterojunction diodes. The corresponding results are illustrated in chapter 6 .

Furthermore, the photosensitivity (chapter 7) as well as the AC properties (chapter 8) of the MASS heterojunction systems were investigated in order to verify or even extend the developed conduction models.

Finally, this thesis is completed by a brief conclusion as well as an outlook on possible improvements in future experiments (chapter 9). 


\section{Chapter 2}

\section{Fundamentals of MASS diode structures}

\section{$2.1 \quad$ Introduction}

In this thesis, the Metal-Amorphous Semiconductor-Semiconductor (MASS) heterostuctures were fabricated according to the assembly sketched in figure 2.1. A crystalline semi conducting substrate, e.g. silicon, zinc oxide or silicon carbide, is covered by a grown thin film of an amorphous or disordered semiconductor, i.e. tetrahedral amorphous carbon (ta-C) or turbostratic boron nitride (t-BN), and metal Schottky contacts (i.e. Au or $\mathrm{Cr} / \mathrm{Au}$, see section 3.6) evaporated on top.

In the following, the MASS diode structure will be described providing basic information about its features as well as the fabrication process.

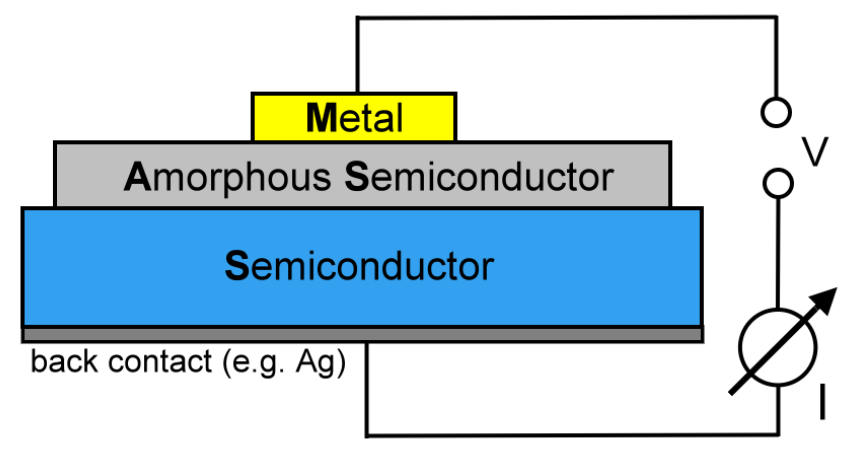

Figure 2.1: Schematic of a Metal-Amorphous Semiconductor-Semiconductor (MASS) heterostructure. 


\subsection{Semiconducting Substrates}

As semiconductor material of the MASS diode structure, single crystalline semiconducting substrates like silicon $(\mathrm{Si})$, silicon carbide $(\mathrm{SiC})$ and zinc oxide $(\mathrm{ZnO})$ were used. The subsequent sections will provide some basic information about the substrates e.g. resistivity, crystalline orientation and lattice parameters as well as origin. The corresponding information about all substrates investigated in this work are summarized in table 2.1 .

\begin{tabular}{|c|c|c|c|c|}
\hline substrate & $\mathbf{n}^{++}-\mathbf{S i}$ & p-Si & $\mathrm{ZnO}$ & $\mathrm{SiC}$ \\
\hline structure & diamond & diamond & wurtzite & 6H-polytype \\
\hline $\begin{array}{l}\text { lattice } \\
\text { constant }\end{array}$ & $\mathrm{a}=0.543 \mathrm{~nm}$ & $\mathrm{a}=0.543 \mathrm{~nm}$ & $\begin{array}{l}\mathrm{a}=0.325 \mathrm{~nm} \\
\mathrm{c}=0.521 \mathrm{~nm}\end{array}$ & $\begin{array}{c}0.30806 \mathrm{~nm} \\
\mathrm{c}=1.51173 \mathrm{~nm}\end{array}$ \\
\hline band-gap [eV] & 1.17 & 1.17 & 3.37 & 3.0 \\
\hline effective mass $\left[m_{e}\right]$ & 1.09 & 0.81 & 0.24 & 0.42 \\
\hline orientation & 111 & 100 & 0001 & 0001 \\
\hline dopant element & phosphorus & boron & - & nitrogen \\
\hline resistivity $[\Omega \mathrm{cm}]$ & $0.005-0.01$ & 10 & $100-10000$ & $0.05-0.1$ \\
\hline
\end{tabular}

Table 2.1: Basic parameters of semiconducting substrates investigated in this work.

\subsubsection{Silicon}

Silicon ( $\mathrm{Si}$ ) is an indirect semiconductor with a cubic diamond lattice structure as shown in figure 2.2. In this crystalline structure, the $\mathrm{Si}$ atoms are coordinated in a fourfold geometry with $\mathrm{sp}^{3}$-bonds between the atoms and a tetrahedral alignment. It can easily be doped p- as well as n-type during growth or subsequently by ionimplantation. While p-type doping is almost solely achieved by the incorporation of boron as group III acceptor element, n-type doping is typically realized using the group $\mathrm{V}$ elements phosphorous or arsenic as donor atoms. In addition, silicon

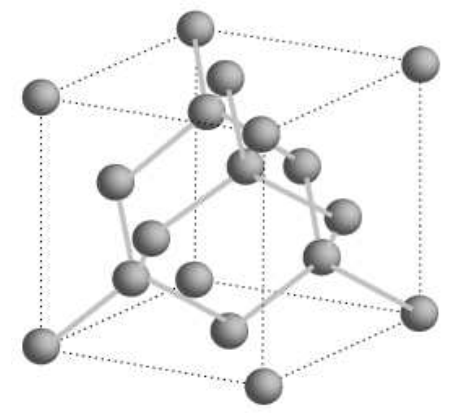

Figure 2.2: Cubic diamond structure of the silicon lattice. The Si atoms are coordinated in a fourfold geometry with $\mathrm{sp}^{3}$ bonds between the atoms and a tetrahedral alignment (from [64]).

is also sensitive to light leading to diverse applications in the fields of solar cell [65], photo diode [66] and sensor technology. Moreover, intrinsic silicon crystals can be fabricated at very high purity levels with contaminant ratios below $10^{-15} \mathrm{~cm}^{-3}$ 67] especially regarding transitions metals with high diffusion rates like $\mathrm{Cu}$ which 
tend to interrupt the performance of Si-based technology in a severe way. Furthermore, various detailed measurements and simulations were performed by many groups in order to determine the material properties such as the lattice constant of $a=0.543 \mathrm{~nm}$ or its band structure with a small, indirect bandgap of $1.17 \mathrm{eV}$ at a temperature of $300 \mathrm{~K}$ [68]. The effective masses of electrons and holes are $1.09 \mathrm{~m}_{\mathrm{e}}$ [69] and $0.81 \mathrm{~m}_{\mathrm{e}}$ [70] at $300 \mathrm{~K}$.

In this thesis, boron doped single crystalline 4 inch Si-wafers with (100) orientation were purchased from Wacker Siltronic. These wafers were cut out a single crystal grown by the most common growth techniques for Si single crystals, the Czochralski (CZ) growth method [71], and doped with boron. They are one side polished and exhibit p-type conductivity as well as a resistivity of approximately $10 \Omega \mathrm{cm}$. Furthermore, the thickness was specified to $625 \pm 10 \mu \mathrm{m}$.

In addition to the low doped p-type silicon, heavily doped n-type silicon wafers were also used in this thesis. With a diameter of 4 inch and a thickness of $625 \pm 20 \mu \mathrm{m}$ the dimensions of the wafers were almost identical to the p-type Si. These wafers are (111) oriented and were heavily doped with phosphorous in order to achieve an almost metallic conduction due to a very low resistivity of approximately $(0.005-$ 0.01) $\Omega \mathrm{cm}$. Therefore, these substrates were primely used for the fabrication of amorphous semiconductor reference samples in order to determine the electrical properties of those thin films (see chapter 6 and 8 ).

\subsection{2 n-type $6 \mathrm{H}-\mathrm{SiC}$}

Silicon carbide $(\mathrm{SiC})$ is a polymorphic wide band-gap semiconductor and exists in about 250 different structures including crystalline as well as amorphous structures. The crystalline part of these structures are called polytypes. These polytypes are variations of the same chemical composition exhibiting identical stoichiometry and mass density of $\rho=3.21 \mathrm{~g} / \mathrm{cm}^{3}[72,73]$. Furthermore, all of these structures are identical in two dimensions but they differ in the third which can be interpreted as a deviation in stacking order of the different crystals. Therefore, the different polytypes originate from different stacking sequences of the layers. As a result, the unit cell sizes and alignment configurations vary from one polytype to another. Despite the great number of polytypes, most of scientific as well as commercial interest is focused on $3 \mathrm{C}-, 4 \mathrm{H}$ and $6 \mathrm{H}-\mathrm{SiC}$ configurations.

3C-SiC (also: $\beta$-SiC) is the only cubic polymorph and has a zinc blende crystal structure. It is mostly referred to as $3 \mathrm{C}-\mathrm{SiC}$ because of the 3 layer stacking sequence $(\mathrm{ABC})$ and cubic structure. Despite many different approaches, there are still no single crystalline substrates available up to now.

Furthermore, $\alpha$-SiC (2H-SiC) and the related polytypes $4 \mathrm{H}-\mathrm{SiC}$ and $6 \mathrm{H}-\mathrm{SiC}$, exhibit a hexagonal unit cell with $2 \mathrm{H}-\mathrm{SiC}$ actually forming a wurtzitic structure with an $\mathrm{ABAB}$ stacking sequence. This periodicity is doubled in $4 \mathrm{H}-\mathrm{SiC}$ and even tripled in $6 \mathrm{H}-\mathrm{SiC}$ with corresponding stacking sequences of ABCB $(4 \mathrm{H})$ and ABCACB $(6 \mathrm{H})$ including the formation of additional cubic lattice sites inside the structures $(4 \mathrm{H}: 1$, 


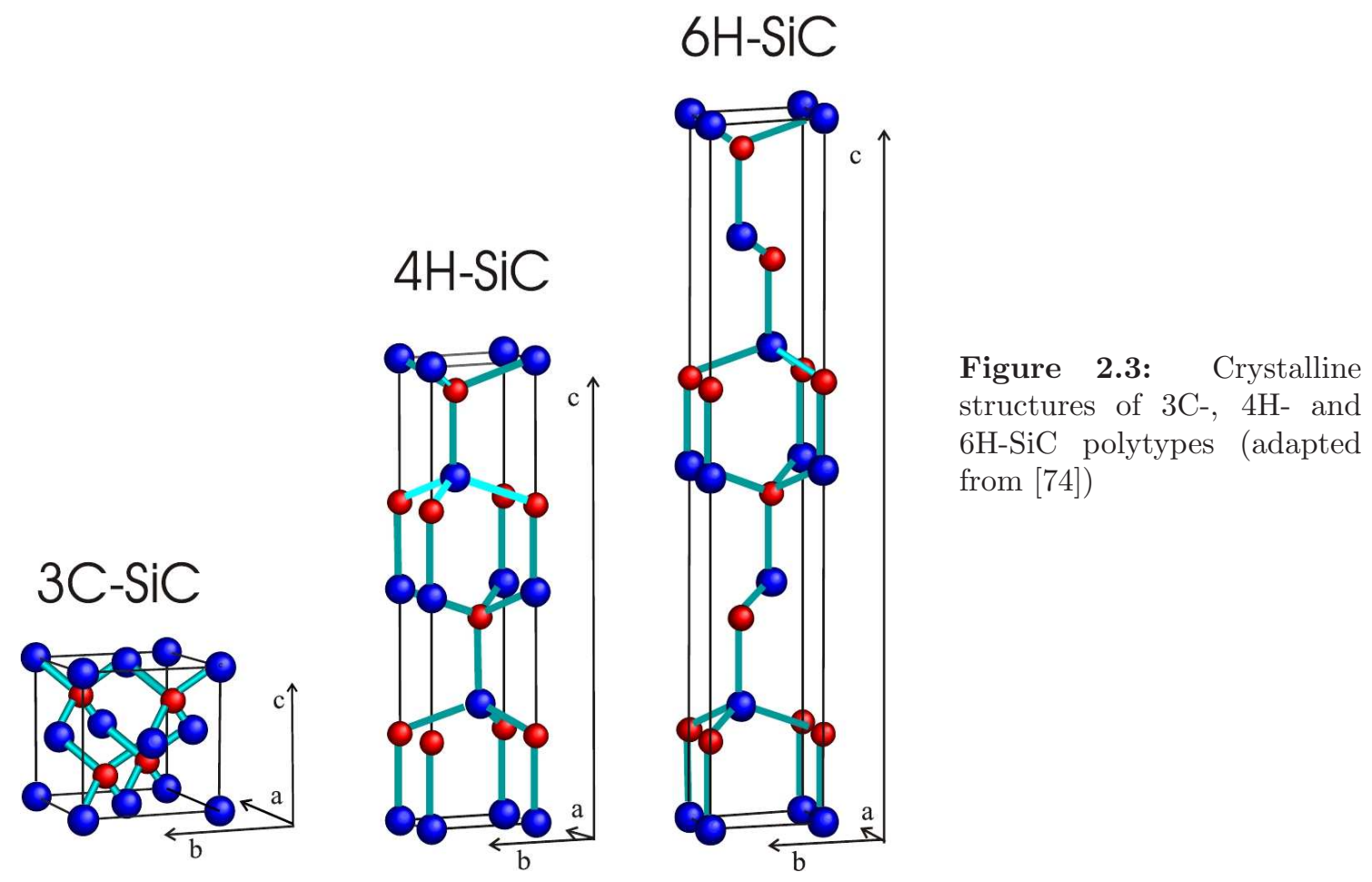

6H: 2). All of these crystal structures are sketched in figure 2.3.

The hexagonal polytypes $4 \mathrm{H}$ and $6 \mathrm{H}-\mathrm{SiC}$ are commercially fabricated in single crystalline wafers up to $100 \mathrm{~mm}$ in diameter and have been investigated in great detail over the past five decades [72]. The most prominent and commercially used growth technique of bulk $\mathrm{SiC}$ single crystals is physical vapor transport via seeded sublimation [20, 75]. In the past two decades the main focus was directed towards the reduction of defects during the growth process of $\mathrm{SiC}$ single crystals (boules). In this context, a special type of defect, so called micropipes, was investigated by many research groups. [18 27]. Micropipes are bulk defects (voids) emerging during growth of a $\mathrm{SiC}$ single crystal and propagating through the length of the boule, starting with the seed crystal. Due to a lower solubility in comparison to dopant atoms like $\mathrm{N}$ or $\mathrm{Al}$ [21], impurity transition metal atoms, e.g. vanadium, accumulate in the vicinity of micropipes leading to decreased blocking voltages in Schottky diodes or high leakage currents in $\mathrm{SiC}$ based high power devices, e.g. thyristors.

Regarding the general properties of silicon carbide, it can be doped n-type using nitrogen or phosphorus [76, 77] as well as p-type due to incorporation of aluminum, boron, gallium or beryllium [76, 78], with $\mathrm{N}$ and $\mathrm{Al}$ being the most popular dopant in commercially fabricated $\mathrm{SiC}$. $\mathrm{N}$ doping results in a blue or even green color whereas $\mathrm{Al}$ doping leads to black crystals [72]. The doping of $\mathrm{SiC}$ single crystals itself is realized by the incorporation of doping atoms during the growth process or, afterwards, by ion implantation.

In this thesis, single crystalline 2" wafers of nitrogen doped $6 \mathrm{H}-\mathrm{SiC}$ were purchased from SiCrystal AG. The hexagonal structure of $6 \mathrm{H}-\mathrm{SiC}$ exhibits a large unit cell 
with lattice parameters of $a=0.30806 \mathrm{~nm}$ and $c=1.51173 \mathrm{~nm}$ 73. The band-gap is in the range of $E_{g}=3.0-3.05 \mathrm{eV}$ [19, 73, 79] and effective electron masses were determined to $m_{l}=2.0(2) m_{0}$ (longitudinal) and $m_{t}=0.42(2) m_{0}$ (transversal, along the c axis) [80]. The wafers are (0001) oriented and exhibit a resistivity of $0.06-0.1 \Omega \mathrm{cm}$ as well as a thickness of $250 \pm 25 \mu \mathrm{m}$. All of them were one side polished (Si-face) and the micropipe density was specified to a value below $100 \mathrm{~cm}^{-2}$. Prior to the actual sample preparation, the wafers were cut into pieces of $5 \times 5 \mathrm{~mm}^{2}$ and $5 \times 10 \mathrm{~mm}^{2}$ using a dicing saw.

\subsubsection{Zinc-oxide $(\mathrm{ZnO})$}

Zinc Oxide $(\mathrm{ZnO})$ is a II-VI wide band gap semiconductor crystallizing in a cubic zincblende or a hexagonal wurtzite structure. The wurtzite structure is the most common form of $\mathrm{ZnO}$ due to its stability at ambient conditions. The zincblende structure of $\mathrm{ZnO}$, in contrast, is only formed at certain conditions on substrates with cubic lattice structures. In either case, $\mathrm{Zn}$ and $\mathrm{O}$ atoms are coordinated in a fourfold geometry leading to tetrahedrally aligned bonds. These bonds are largely ionic due to the discrepancy in electron affinity and electro negativity between zinc and oxygen.

A detailed review of $\mathrm{ZnO}$ and its properties are given by Özgür et al. [81] as well as Pearton et al. [82]. The wurtzite $\mathrm{ZnO}$ structure is shown in figure 2.4 and possesses lattice parameters of $a=0.325(1) \mathrm{nm}$ and $c=0.521(1) \mathrm{nm}$ [81, 82]. In addition,

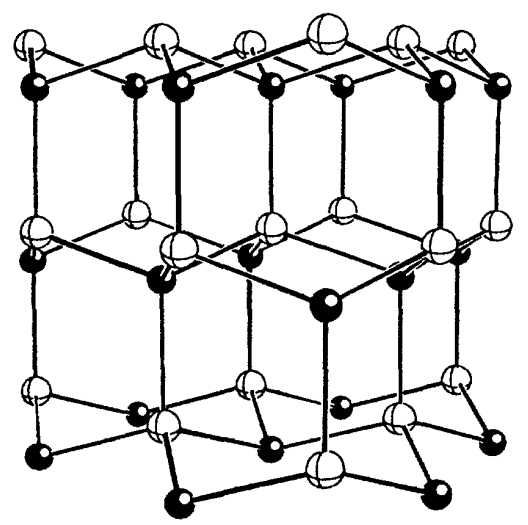

Figure 2.4: Wurtzite structure of $\mathrm{ZnO}$ (taken from reference [83]. Copyright 1993 by The American Physical Society.)

wurtzite $\mathrm{ZnO}$ exhibits a large direct band-gap of $E_{g}=3.37 \mathrm{eV}$ determined by various experiments [81]. Therefore, $\mathrm{ZnO}$ shows several favorable properties such as high breakdown voltages, the ability to sustain large electric fields as well as high thermal stability. This leads to applicability of $\mathrm{ZnO}$ in high-temperature and highpower operation devices.

In general, intrinsic $\mathrm{ZnO}$ has a n-type character, even without any intentional doping during or after growth of the crystals. This is typically caused by irregularities 
in stoichiometry of the crystal like oxygen vacancies or interstitial $\mathrm{Zn}$ atoms leading to intrinsic n-type conduction. In this context, the effective electron mass has a value of $0.24 \mathrm{~m}_{e}$ [82].

The n-type doping can easily be controlled by substitution of Zn atoms with groupIII elements such as Al, Ga, In. Alternatively, this can also be achieved by a substitution of oxygen with group-VII elements like chlorine or iodine [84]. In addition, its worth mentioning that p-type doping was only reported by a few groups and is almost impossible in terms of commercial use due to the absence of a sufficient quantity regarding low level acceptor states as well as the presence of intrinsic donors states [81].

The growth of single crystals can be realized by a variety growth methods such as a gas transport method (e.g. vapor-phase deposition), synthesis of crystals from a melt or hydrothermal growth. The first two methods are difficult to realize on a commercial scale based on the facts that $\mathrm{ZnO}$ possesses a high vapor pressure (melt) and the gas transport is difficult to control, in particular on a large scale. Therefore, the only growth method remaining and controllable on a larger scale is the hydrothermal growth.

An example of this growth technique is the Chemical Vapor Transport (CVT) growth method. It is described in detail by Takahashi et al. [85]. In principle, this hydrothermal growth method is based on the formation of a crystal from an aqueous solutions at high-temperatures and high vapor pressures. The single-crystalline character is achieved using appropriate seed crystals. The setup, usually used for this growth method, is a thick-walled steel cylinder, a so called autoclave. It is equipped with a hermetic seal resistive to high temperatures and pressures. In order to avoid corrosion of the autoclave's inner cavity due to interaction with the solvents used in the process, protective inserts are often used.

In this particular case of CVT, the actual crystallization vessel is an inner platinum (Pt) container inserted into the autoclave. As only a small volume of the autoclave is occupied by this kind of floating type insert, an appropriate amount of distilled Water is supplied in the space between the autoclave cavity and the inner container for pressure balance reasons. The inner Pt container itself is filled with a solution made up of $\mathrm{H}_{2} \mathrm{O}$, a nutrient ( $\mathrm{ZnO}$ powder) and a small amount of $\mathrm{KOH}$ and $\mathrm{LiOH}$. The latter are called mineralizers facilitating the transport of insoluble nutrient like $\mathrm{Zn}$ to a seed crystal on the basis of a reversible chemical reaction. The growth of $\mathrm{ZnO}$ single crystals takes place at temperatures of $300-400^{\circ} \mathrm{C}$ and pressures of 80-100 MPa. During growth, $\mathrm{ZnO}$ powder reacts with hydrogen forming gaseous $\mathrm{Zn}$ and $\mathrm{H}_{2} \mathrm{O}$ at one side of the furnace. Then, the gas phase $\mathrm{Zn}$ is transported by hydrogen and another, inert gas in the direction of the seed crystal on the opposite side of the furnace. There, the $\mathrm{Zn}$ is reoxidized by a reaction with oxygen gas leading to the growth of $\mathrm{ZnO}$ on seed crystals. In the process, all gases are supplied through separate tubes.

Compared to other semiconductor single crystal growth methods, CVT is relatively slow at a rate of approximately $0.2 \mathrm{~mm}$ per day. The grown crystals exhibit high 
crystallinity but contain a significant percentage of $\mathrm{Fe}$ and $\mathrm{Al}$, in terms of the effect in semiconductors, as well as small amounts of $\mathrm{K}$ and $\mathrm{Li}$ in the ppm range. Fe and $\mathrm{Al}$ possibly originate from impurities in the original $\mathrm{ZnO}$ powder. The origin of $\mathrm{K}$ and Li impurities, on the other hand, can be pinpointed to the use of mineralizers. Depending on the exact percentage of these metallic impurities, the electrical properties of hydrothermally grown single crystals can vary significantly. An image of a typical 2" $\mathrm{ZnO}$ single crystal is shown in figure 2.5. The typical dimensions of

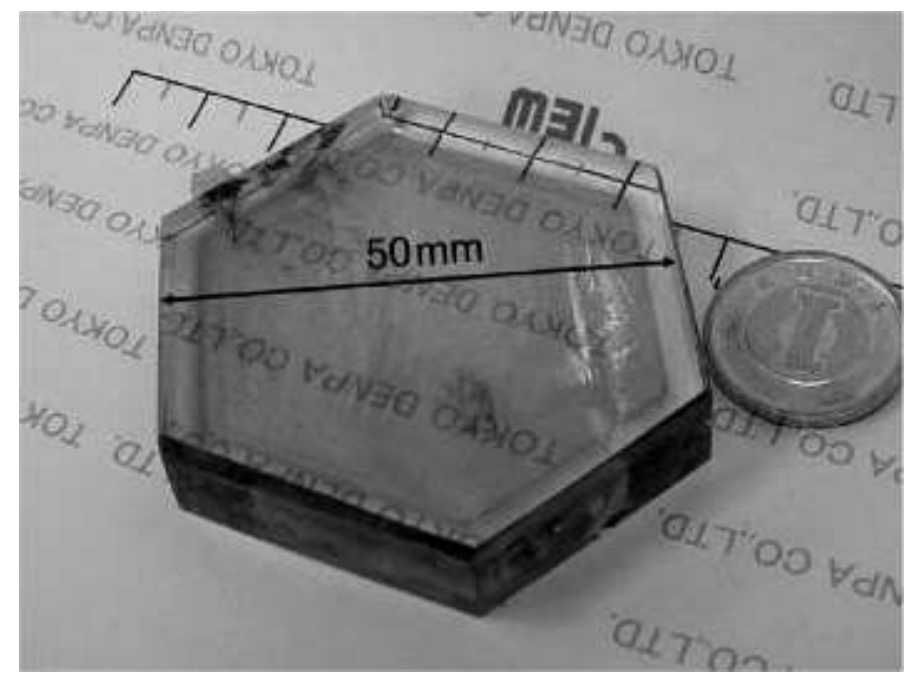

Figure 2.5: Typical 2" $\mathrm{ZnO}$ single crystal grown by a hydrothermal method (taken from [85]).

wafers sliced out of such a bulk crystal are 2 inch in diameter and $0.5 \mathrm{~mm}$ thickness. They are polished by a chemical mechanical polishing technique (CMP) and damage is caused at the surface of the wafers. Therefore, they are annealed for 4 hours at $1100^{\circ} \mathrm{C}$ in air and a pressure of $1 \mathrm{~atm}$.

In this thesis, hydrothermal grown single crystals with one sided Zn-face polish and an (0001) orientation were purchased from Crystec. Due to the growth process, this kind of $\mathrm{ZnO}$ contains several impurities such as $\mathrm{Mg}, \mathrm{Al}, \mathrm{Si}, \mathrm{Ti}, \mathrm{Cu}, \mathrm{Fe}, \mathrm{Ca}$ and $\mathrm{Ag}$ at an unknown percentage between 0.002-0.005\%. All samples were cut out of a single crystalline wafer and have dimensions of $5 \times 10 \mathrm{~mm}^{2}$ as well as a thickness of $500 \mu \mathrm{m}$. 


\subsection{Ohmic back contact preparation}

Another important part of the sample preparation process is prevention of unnecessary interference barrier build up in terms of the electronic structure often originating from the preparation steps of metal-semiconductor contacts. In this thesis, metal contacts were evaporated on the back side of the semiconductor substrates and annealed afterwards in order to achieve a minimal contact resistance and an ohmic current-voltage characteristic of the substrates. Annealing procedures, if necessary, were performed in an rapid thermal processing (RTP) furnace at certain temperatures and durations. The basic information about ohmic back contact preparation of each substrate are summarized in table 2.2. The electrical characterization setup is described in detail in section 4.2 and actual measurements and characterization of the particular substrates are discussed in chapter 6 .

\begin{tabular}{|c|c|c|c|}
\hline semiconductor & contact & annealing conditions & references \\
\hline $\mathrm{p}-\mathrm{Si}$ & $\mathrm{Al}$ & $900^{\circ} \mathrm{C}(\mathrm{Ar}), 1 \mathrm{~min}, \mathrm{RTP}$ & {$[86,87]$} \\
\hline $6 \mathrm{H}-\mathrm{SiC}$ & $\mathrm{Ni}$ & $950^{\circ} \mathrm{C}, 1 \mathrm{~min}, \mathrm{RTP}$ & {$[88,89]$} \\
\hline $\mathrm{ZnO}$ & $\mathrm{Ti} / \mathrm{Au}$ & no annealing & {$[90,91]$} \\
\hline
\end{tabular}

Table 2.2: Summary of ohmic back contact preparation used in this work.

\subsubsection{Si based MASS diodes}

In the beginning of silicon based integrated circuit (IC) fabrication, aluminum contacts were used as ohmic contacts as well as rectifying "Schottky contacts". In this context, the publication of H.C. Card provides a brief review as well as a detailed investigation of interface behavior in Al-Si contacts regarding different types of heat treatment [86]. Right after the preparation process, fresh $\mathrm{Al}$ contacts on Si are very sensitive to surface properties of the Si substrate like surface cleaning, possible residual contaminants and evaporation conditions. In this context, an as deposited $\mathrm{Al}$ contact on p-type $\mathrm{Si}$ which contained a thin oxide surface layer $(\approx 2 \mathrm{~nm})$ leads to rectifying characteristics of this heterostructure. However, long-time "lowtemperature" treatment below or around $300^{\circ} \mathrm{C}$ of these samples led to a significant reduction of Schottky barrier height between $\mathrm{Al}$ and $\mathrm{Si}$ determined by capacitancevoltage measurements. An increase in temperature up to the eutectic temperature of $550^{\circ} \mathrm{C}$ led to further decrease of the barrier height. In this context, the final barrier height does not depend on duration of the annealing procedure as long as a minimal time of $1 \mathrm{~min}$ is reached. Still, the barrier height is a function of the cooling rate after annealing [87]. Above the eutectic temperature, further penetration of $\mathrm{Al}$ into the interface is observed. The resulting current-voltage characteristics of $\mathrm{Al} / \mathrm{p}$-type $\mathrm{Si}$ contacts at room temperature regarding the different annealing steps are illustrated in figure 2.6. In addition, it has also been shown that the electrical properties of $\mathrm{Al}$ contacts on $\mathrm{n}$-type $\mathrm{Si}$ progress in the opposite direction under the 


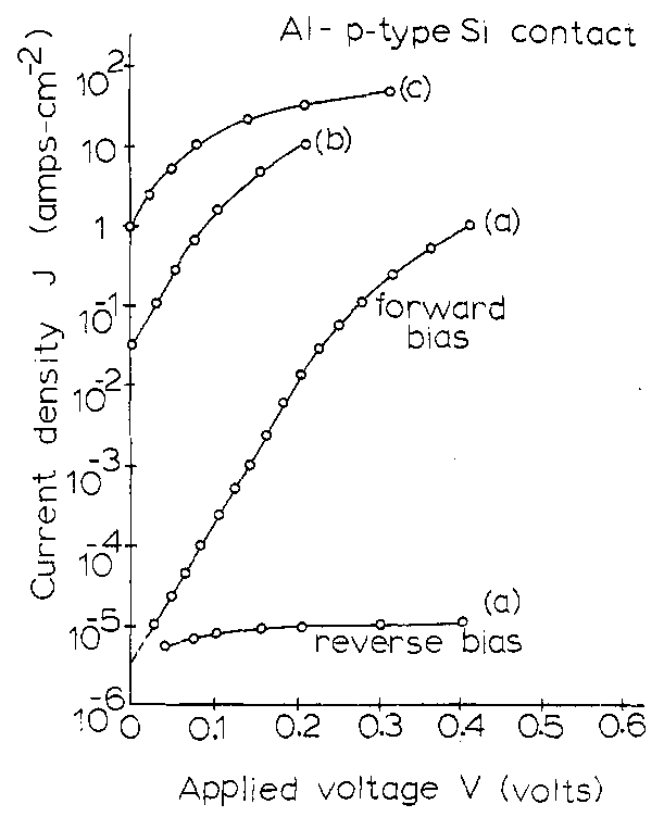

Figure 2.6: Current-voltage characteristics at room temperature of $\mathrm{Al} / \mathrm{p}$-type $\mathrm{Si}$ contacts, including a very thin oxide layer of $\mathrm{d}=2 \mathrm{~nm}$, at different annealing conditions. (a) The Al contacts are freshly evaporated. (b) The conductivity in forward bias increases due to aging and low temperature annealing at $T \leq 300^{\circ} \mathrm{C}$. (c) The characteristic becomes ohmic after annealing at $550^{\circ} \mathrm{C}$. (taken from reference [86], (c)1976 IEEE)

same conditions as low doped n-type silicon substrates form Schottky barriers of up to $0.9 \mathrm{eV}$ in barrier height.

In the past decades, associated with advancing progress in device processing, metal silicides replaced $\mathrm{Al}$ as a contact material because of considerable lower diffusion rates into unintended areas of the devices, e.g. during high temperature processing steps.

However, Al contacts were chosen in this work due to the simplicity of contact preparation compared to alternative methods.

In a first step, the Si substrate was mounted inside a Leybold UNIVEX 350 evaporation chamber and a shadow mask with two rectangular bars of $10 \mathrm{~mm} \times 2 \mathrm{~mm}$ divided by a distance of $3 \mathrm{~mm}$, shown in figure 2.7, was applied to the rough backside of the Si substrates. After reaching an operating base pressure of about $5 \cdot 10^{-6}$ mbar, the back side of the p-type Si substrates was sputter-cleaned for (10-15) min using Ar ions at an energy of $2 \mathrm{keV}$ and an etching rate of (4-5) nm/min. The sample holder was rotated constantly in order to provide a homogeneous sputter rate all over the sample. After that, the Al deposition was started using an electron-beam evaporation unit. The e-beam had an energy of $7 \mathrm{keV}$ and was swept dynamically over the Al surface inside the melting pot. The resulting evaporation rate was $4 \AA / \mathrm{s}$. Meanwhile, the shutter right in front of the sample was closed and the first $15 \mathrm{~nm}$ were evaporated blind in order to avoid contamination of the contacts with oxide or other contaminants. Then, the actual deposition was started by opening the shutter 


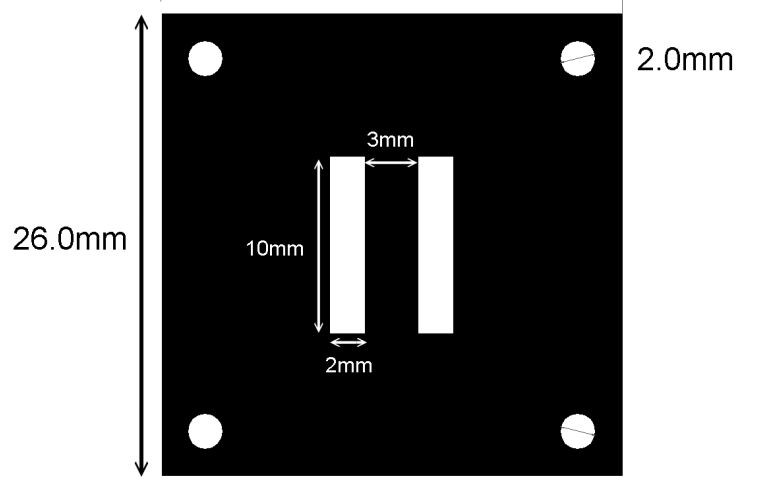

Figure 2.7: Shadow mask applied to the backside of the silicon substrates prior to aluminum deposition.

and $250 \mathrm{~nm} \mathrm{Al}$ were homogeneously deposited on the rotating sample.

The contacts obtained from this procedure exhibited a comparatively high resistivity but were almost ohmic contacts. Therefore, the substrates were additionally annealed in a rapid thermal processing (RTP) furnace, an ANNEALSYS AS100 TOWER, at $900^{\circ} \mathrm{C}$ under Argon atmosphere. The Ar-flow was inserted at a flowrate of about $500 \mathrm{sccm}$ and the heating ramp was set to $30 \mathrm{~s}$ until the annealing temperature was reached. In order to ensure a sufficient reaction, an annealing time of 1 min was chosen. In a final step, the chamber was cooled down. In doing so, a temperature of approximately $300^{\circ} \mathrm{C}$ was reached in about $1 \mathrm{~min}$. Compared to the results from Card, these annealing steps ensure a high diffusion rate of the $\mathrm{Al}$ into Si resulting in a sufficient decrease of barrier height and a strong increase in carrier concentration due to the formation of Al-Si compounds inside of the interface region.

As a result, these contacts exhibit a low resistive and ohmic characteristic. The corresponding current-voltage characteristics of the different contacts are shown and discussed in detail in section 6.3 .

\subsubsection{SiC based MASS diodes}

$\mathrm{SiC}$ is a suitable material for high power and high temperature device application based on its large band-gap in combination with a variety of possible doping and favorable thermal properties. Such devices often demand low resistive ohmic contacts as a key feature to be addressed.

In this context, Crofton et al. gave a detailed review on ohmic contacts to $\mathrm{SiC}$ in [89]. As no p-type $\mathrm{SiC}$ were used in this thesis, only contact materials applied to n-type $\mathrm{SiC}$ will be discussed at this point. The basic concept in the fabrication of all ohmic contacts on $\mathrm{n}$-type $\mathrm{SiC}$ is the annealing of an, at first, refractory metal causing a formation of silicides with a lowered Schottky barrier height at the metal-SiC interface. There have been many reports on the formation of ohmic contacts based on a variety of transition metals like Mo, Co, Hf, Ta, W and several Ti compounds 
92 96. However, contacts made up of Ni are most frequently used and well investigated by several groups [93, 96 98].

In an earlier work, Crofton et al. investigated nickel contacts on $6 \mathrm{H}-\mathrm{SiC}$ operating at high temperatures as well as the effects of different annealing temperatures [88]. After annealing for $2 \mathrm{~min}$ at $950^{\circ} \mathrm{C}$ in vacuum, these contacts exhibit low specific contact resistance and additionally provides good electrical and physical stability fitting the requirements of long-term high temperature devices as well as high power device applications. In principle, a successful preparation of low resistive ohmic contacts is based on a reaction of $\mathrm{Ni}$ and $\mathrm{SiC}$ at sufficient high annealing temperatures above $900^{\circ} \mathrm{C}$ and short-term annealing times between 1 and 5 min leading to the formation of a metal-rich nickel silicide layer of $\mathrm{Ni}_{2} \mathrm{Si}$. Furthermore, this reaction is accompanied by a migration of $\mathrm{C}$ away from the interface and accumulation on the free silicide surface as well as possible formation of vacancies in the interface region during the reaction of $\mathrm{Ni}$ and $\mathrm{SiC}$. Both processes might facilitate the ohmic behavior of the contacts. All of these results have been confirmed and complemented by

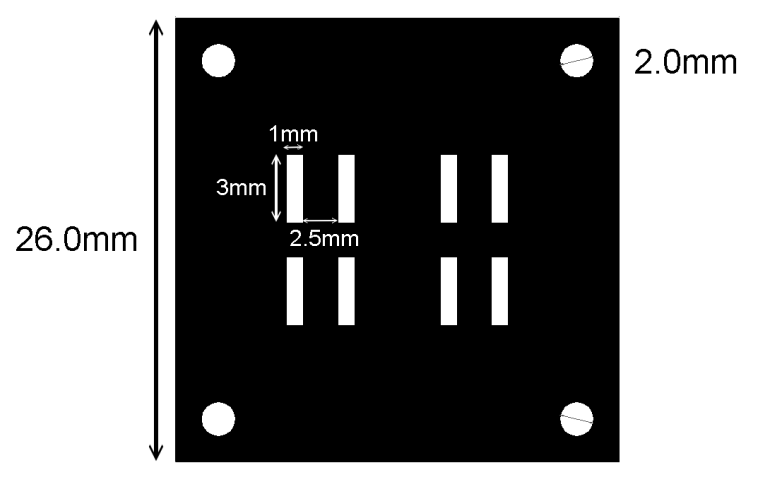

Figure 2.8: Shadow mask applied to the backside of the silicon substrates prior to nickel deposition.

Liu et al. and several other groups 93, 96 98.

In addition, Marinova et al. suggested the deposition of $\mathrm{Ni} / \mathrm{Si}$ multilayers instead of pure $\mathrm{Ni}$ in order to lower the accumulation rate of carbon as it might be a source of contact degradation at high temperatures [99].

In this thesis, pure nickel contacts were used as back contact material. Regarding the evaporation and annealing processes during contact preparation, identical setups compared to the Si substrates in the section above were used. Due to the sample size, the bar-patterns of the shadow mask are significantly smaller with dimensions of $3 \mathrm{~mm} \times 1 \mathrm{~mm}$ divided by a distance of $2.5 \mathrm{~mm}$ as it is shown in figure 2.8 . During evaporation nickel contacts with a thickness of $100 \mathrm{~nm}$ were homogeneously deposited at a rate of $(4-5) \AA / \mathrm{s}$ onto the $6 \mathrm{H}-\mathrm{SiC}$. They were annealed at $900^{\circ} \mathrm{C}$ for about 1 min under vacuum conditions $\left(1 \cdot 10^{-6}\right.$ mbar $)$ in the rapid thermal processing (RTP) furnace. As a result, the resistance was significantly lowered by two orders of magnitude compared to as grown Ni contacts (see section 6.4.1). 


\subsubsection{ZnO based MASS diodes}

Zinc oxide has attracted much interest in the fields of UV light-emitters, high-power electronics, surface acoustic wave devices and many more. Therefore, ohmic as well as rectifying Schottky contacts have been extensively investigated by various groups in order to improve their quality and match application demands [82].

In this context, Ip et al. gave a detailed review of ohmic and Schottky contacts on $\mathrm{ZnO}$ [91]. Regarding rectifying Schottky contacts, metals such as Au, Ag, Pt and Pd form suitable Schottky barriers according to several publications, mainly focusing on $\mathrm{Au}$ and Ag.

Focusing on ohmic conduction of evaporated metal contacts, there are many reports in literature on low resistive contacts made up of $\mathrm{Ti} / \mathrm{Al}, \mathrm{Ta} / \mathrm{Au}$ and $\mathrm{Pt}$ based contacts as well as on more complex Ti/Al/Pt/Au structures [82, 100, 101].

Furthermore, the formation of ohmic contacts on the basis of titanium and gold (Ti/Au) has attracted interest by some groups and was first studied by Kim et al. [90]. They reported on the electrical properties of ohmic Ti/Au contacts on rf magnetron sputtered and $\mathrm{Al}$ doped $\mathrm{ZnO}$. They showed that annealing of $30 \mathrm{~nm} \mathrm{Ti} / 50 \mathrm{~nm}$ $\mathrm{Au}$ contacts at $300{ }^{\circ} \mathrm{C}$ under nitrogen atmosphere for 1 min led to linear current voltage characteristics up to $5 \mathrm{~V}$ and a specific contact resistance of $2 \times 10^{-4} \Omega \mathrm{cm}^{2}$. In further work, they also showed the formation of ohmic $\mathrm{Ti} / \mathrm{Au}$ contacts on n-type $\mathrm{ZnO}$ bulk crystals with a specific contact resistance of $5 \times 10^{-5} \Omega \mathrm{cm}^{2}$.

In principle, a Ti-oxide layer is formed due to the chemical reactivity of $\mathrm{Ti}$ and oxygen. There are indications that the formation of the Ti-O phase is fed by outdiffusion of oxygen from the $\mathrm{ZnO}$ leading to the accumulation of oxygen vacancies near the $\mathrm{ZnO}$ surface. These vacancies act as donors in the $\mathrm{ZnO}$ increasing the carrier concentration near the surface of $\mathrm{ZnO}$ leading to a formation of ohmic contacts. In this thesis, $\mathrm{Ti} / \mathrm{Au}$ contacts were chosen due to the number of available publications as well as the fact that only a marginal or even no annealing procedure is needed to form ohmic contacts on ZnO. Titanium and Gold were thermally evaporated in a layered structure of (15-20) nm Ti and $60 \mathrm{~nm}$ Au using an identical shadow mask layout as for $\mathrm{SiC}$ substrates (shown in figure 2.8) due to similar sample sizes. These contacts were fabricated at an ambient pressure of $(0.5-1) \cdot 10^{-6}$ mbar in an evaporation chamber of our own work-group which is described in detail in section 2.6 and was also used for the evaporation of the metal contacts of the MASS diode structure. Due to degrading electrical conduction (see section 6.2) only one sample was annealed afterwards under the described conditions. 


\subsection{Amorphous Semiconductors}

The amorphous semiconductor of MASS diodes is made up of a thin film of tetrahedral amorphous carbon (ta-C) or turbostratic boron nitride (t-BN). In this context, detailed reviews on the properties of these kind of materials have been published by Robertson et al. for amorphous carbon (a-C) [102] and by Mirkarimi et al. for boron-nitride (BN) [103]. It has to be noted that ion deposited amorphous (ta-C) or nano-crystalline (t-BN) thin films do not form classical acceptor or donor levels due to the high defect density and therefore cannot be doped like e.g. amorphous silicon. In fact, they show a similar behavior to the "I" part in MIS structures but exhibit a higher conductivity [46, 47].

\subsection{1 tetrahedral amorphous carbon (ta-C)}

The most common microscopical configurations of carbon are graphite and diamond. Graphite consists of six-folded carbon rings stacked in layers based on a threefold coordination of the carbon atoms, the $\mathrm{sp}^{2}$-hybridization. The $\mathrm{sp}^{2}$-hybridized carbon sites are the stable configuration of carbon in a crystalline as well as in an amorphous structure. In contrast, diamond is made up of a metastable fourfold coordination of carbon atoms leading to $\mathrm{sp}^{3}$-bonding of carbons forms tetrahedrons resulting in the diamond structure.

In addition to those crystalline structures, multiple carbon amorphous structures (a:C) can be formed. They contain a mixture of $\mathrm{sp}^{2}$ - and $\mathrm{sp}^{3}$-bonded carbon states and, at some cases, even a small content of $\mathrm{sp}^{1}$-states. Furthermore, hydrogen can be incorporated depending on the fabrication method. This leads to the formation of hydrogenated amorphous carbon (a-C:H). If these amorphous carbons contain a

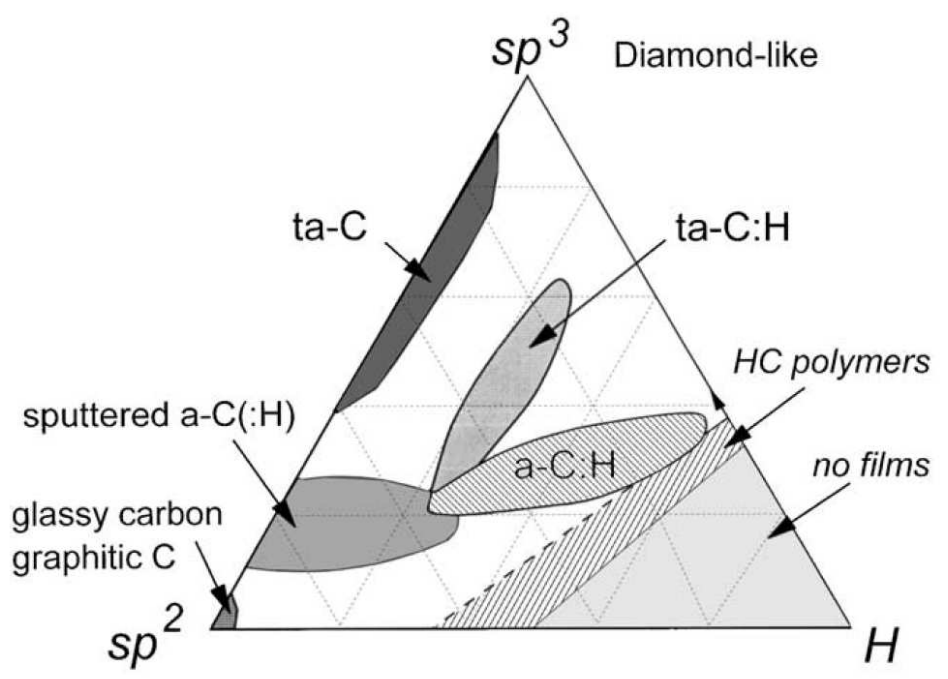

Figure 2.9: Ternary phase-diagram of bonding in amorphous carbon - hydrogen alloys (taken from reference [102], (c)2002, Elsevier). 


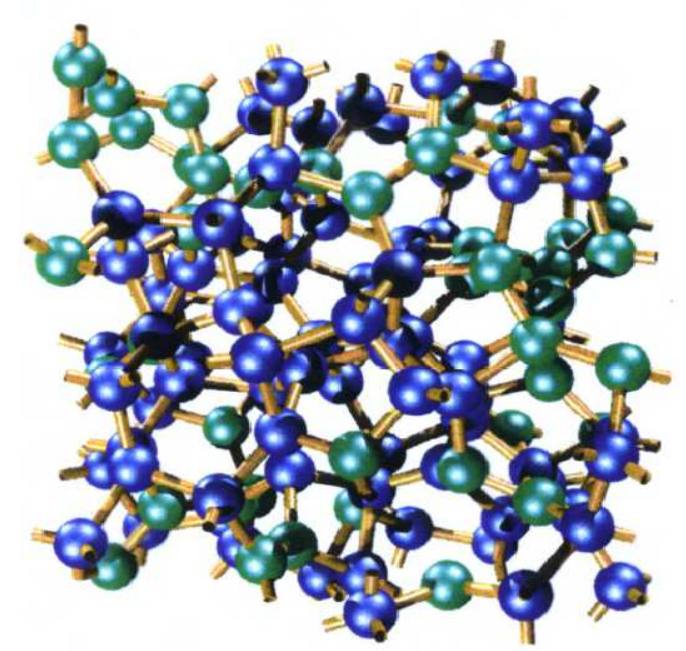

Figure 2.10: Microstructure of tetrahedral-amorphous carbon (ta-C), taken from Niesser et al. [104.

significant percentage of $\mathrm{sp}^{3}$-bonded carbon atoms, this metastable group of a-Cs is called diamond-like carbon (DLC). Depending on the $\mathrm{sp}^{2}$ - to $\mathrm{sp}^{3}$-ratio, there is a variation in density, hardness, permittivity and resistivity. Finally, if these DLC films are hydrogen free, they are called tetrahedral amorphous carbon (ta-C). The corresponding ternary phase diagram of bonding in all of these amorphous carbonhydrogen alloys is displayed in figure 2.9.

As the growth conditions are far away from equilibrium, diamond like carbon thin films can only be synthesized using various methods to create energetic particles. Examples are filtered arc deposition as well as ion beam deposition techniques (e.g. mass selected ion beam deposition (MSIBD)) providing only a low energy fluctuation of the ions and thus well defined thin film properties such as a high resistivity.

In this thesis, all ta-C thin films were grown using MSIBD (see section 2.5) at an ion energy of $100 \mathrm{eV}$ exhibiting a $\mathrm{sp}^{3}$-content of about $80 \%$. As a result, these thin films possess a mass density of about $3 \mathrm{~g} / \mathrm{cm}^{3}$, a high resistivity $\left(\approx 10^{-7} \Omega \mathrm{cm}\right)$ as well as a high hardness ( $>40 \mathrm{GPa}$ ). The typical structural configuration of ta-C, a dispersion of $\mathrm{sp}^{2}$ - and $\mathrm{sp}^{3}$-bonds in an amorphous matrix, is displayed in figure 2.10 . 


\subsection{2 turbostratic boron-nitride (t-BN)}

The crystalline structures of boron nitride are very similar to those formed by carbon. All of them cannot be found in nature and therefore have to be synthesized using boron acids or boron-trioxide as starting material. In comparison to carbon, the phase stability between the hexagonal and the cubic phase is inverted. The $\mathrm{sp}^{3}$ bonded cubic phase, cubic boron-nitride (c-BN), is the stable phase and possesses a zinc-blende structure along with high hardness, a large bandgap and thermal stability similar to diamond. The hexagonal phase, hexagonal boron nitride (h-BN) or "white graphite", is metastable.

In contrast to its carbon counterpart, $\mathrm{h}-\mathrm{BN}$ is not a conducting material but a wide bandgap semiconductor with a bandgap of about $E_{g}=5.2 \mathrm{eV}$ [68]. The sp ${ }^{2}$-bonded layered structure is very similar to graphite. However, the layers are not displaced

(a)

A

$A^{\prime}$

A

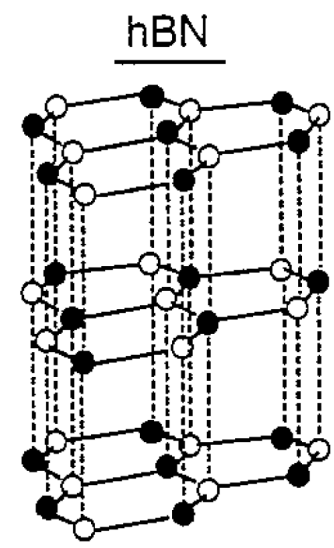

(b)
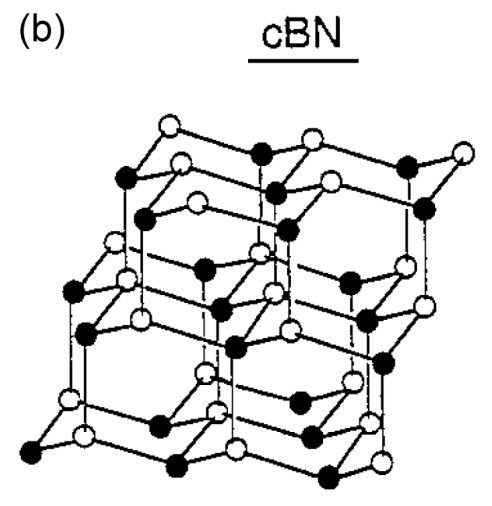

[111]

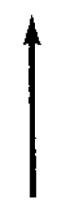

Figure 2.11: Crystalline structures of boron nitride: (a) the $\mathrm{sp}^{2}$-bonded structure of hexagonal boron-nitride (h-BN) is similar to graphite, (b) the $\mathrm{sp}^{3}$-bonded phase, cubic boron-nitride (c-BN), exhibits a zincblende structure similar to diamond. (adapted from [103], (C)1997 Elsevier)

against each other. Instead, the hexagonal rings of the basal planes in h-BN are positioned directly above each other and rotated by $180^{\circ}$ between alternate layers. Both crystalline structures are shown in figure 2.11.

In addition, another hexagonal boron nitride phase often forms during growth attempts of h-BN thin films with ion-beam assisted deposition techniques (IBAD), the so called turbostratic boron-nitride (t-BN). It is a disordered, micro-crystalline form of the hexagonal phase. The two-dimensional in-plane order of the hexagonal structure of the basal planes is almost identical to those in h-BN. However, they are stacked in a random sequence and with random orientation about the c-axis. In more detail, the [0002] planes of t-BN grow perpendicular to a substrate surface, sometimes on top of an additional amorphous interlayer formed by a mixture of B, $\mathrm{N}$ and the substrate material [106]. Furthermore, the orientation of the [0002] directions about the film are random and, in addition, the graphitic layers are randomly rotated about the [0002] directions. As a result, there are no preferred out of plane direction present in such films. All of these aspects are sketched in figure 2.12. An 
(a)

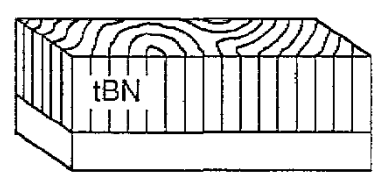

(b)

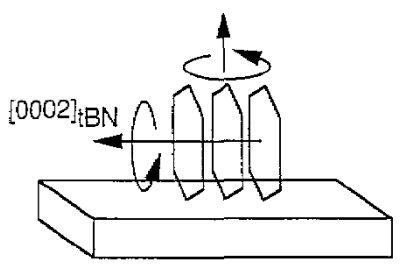

Figure 2.12: Properties of the turbostratic boron-nitride (t-BN) structure: (a) 3D view of basal planes (after McCulloch et al. 105]), (b) [0002] in-plane orientation for t-BN (both pictures taken from [103], (c)1997 Elsevier).

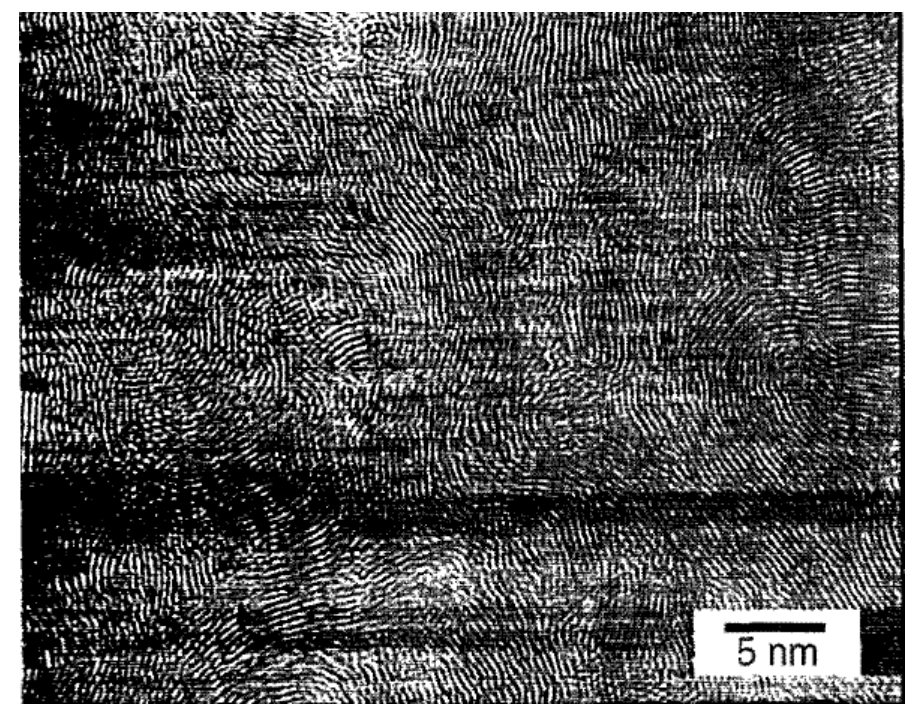

Figure 2.13: In plan HRTEM image of a t-BN thin-film. The basal planes are highly distorted and exhibit a high defect- and dislocation-density. Therefore, they extend only short distances of particular areas (taken from McCarty et al. [108], (c)1996 Elsevier).

additional HRTEM image of a "real" t-BN film in plan view is shown in figure 2.13. In this work, all t-BN films were grown using mass selected ion-beam deposition (MSIBD) at an energy of $100 \mathrm{eV}$. The basic material parameters such as mass density $\rho=2.25 \mathrm{~g} / \mathrm{cm}^{3}$ [107] as well as the lattice parameters $a=0.250(1) \mathrm{nm}$ and $c=0.666(1) \mathrm{nm}$ [68] were obtained from h-BN providing a good approximation due to a similar crystalline structure of the basal planes. A detailed structural analysis of the grown t-BN thin-films in this thesis was performed using transmission electron microscopy (see section 5 ). 


\subsection{Thin-film growth - Mass Selected Ion-Beam Deposition (MSIBD)}

All ta-C and t-BN thin-films of this work were grown using a mass selected ion beam deposition technique at the ion accelerator ADONIS (Anlage zur DepOsition niederenergetischer Ionen auf Substrate). The setup of the ion accelerator ADONIS is described in detail in section 2.5.2. The ions are generated by a hot filament hollow cathode ion source. The functional principle of such an ion source is described in section 2.5.1.

\subsubsection{Ion source}

The setup of the hot filament hollow cathode ion source (also known as Sidenius ion source [109, 110]) is sketched in figure 2.14. All conducting parts are separated by boron nitride isolators. The cathode is made up of a tungsten filament fixed by a graphite and a tantal ring.

In a first step, the filament is heated ( $25 \mathrm{~A}$ at $6 \mathrm{~V}-8 \mathrm{~V})$. As a result, electrons are emitted from the filament due to thermionic emission and accelerated towards the anode, another graphite ring, using a bias of $250 \mathrm{~V}$ between anode and cathode. In

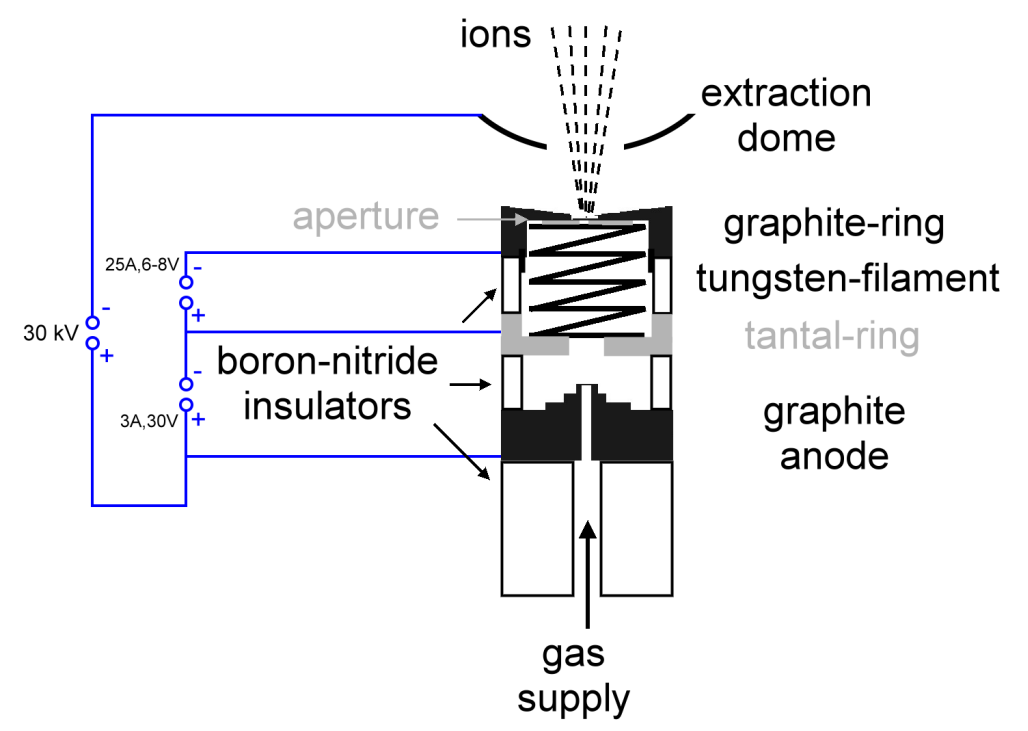

Figure 2.14: Setup of a Sidenius ion source.

order to ignite an ion plasma, a gas (i.e. Ar, $\mathrm{N}_{2}, \mathrm{CO}_{2}$ ) or a gaseous material (i.e. heated $\left.\mathrm{B}_{2} \mathrm{O}_{3}\right)$ is fed through the anode into the hollow part of the ion source in the opposite direction. At a gas pressure of $(1.5-6) \cdot 10^{-5}$ mbar the accelerated electrons cause an electric discharge due to impact ionization. In the process, additional electrons are knocked out of the electron shell and also accelerated towards the anode leading to a cascade effect. Therefore, the anode bias can be reduced to $30 \mathrm{~V}$ 
still maintaining a high electron current. The positively charged ions of the ignited plasma are accelerated towards the cathode. After passing the radial symmetric cathode and leaving the ion source through a circular aperture of (0.7-0.9) $\mathrm{mm}$ in diameter the ions are extracted and accelerated by a high tension of $30 \mathrm{kV}$. 


\subsubsection{The ion-accelerator ADONIS}

The setup of the ion accelerator ADONIS is shown in figure 2.15. First of all, in order to start the MSIBD, the Sidenius ion source is brought to an operational mode (see 2.5.1). It can be fed by numerous gases, i.e. $\mathrm{Ar}, \mathrm{N}_{2}$ or $\mathrm{CO}_{2}$. In addition, solid elements or compounds (i.e. heated $\mathrm{B}_{2} \mathrm{O}_{3}$ ) can be evaporated using an additional furnace. The ion source is charged by a high tension of $30 \mathrm{kV}$. The ions are extracted and accelerated to an energy of $30 \mathrm{keV}$ by the extraction dome on ground potential. The energy straggling is in the range of $5 \mathrm{eV}-10 \mathrm{eV}$ and is based on the energy distribution inside of the ion beam. After extraction the ion beam is focused by an electrostatic lens $(20 \mathrm{kV})$ and separated by mass while passing through a $90^{\circ}$ sector-field magnet. Following the mass-separation, ions with the desired $\mathrm{q} / \mathrm{m}$ ratio pass a $\mathrm{x}-\mathrm{y}$ steering unit, simply two pairs of parallel plate capacitors, and a variable slit which basically defines the mass resolution of the setup.

In a next step, the ion beam is focused with another electrostatic lens as well as a quadrupole lens. An additional electrostatic capacitor with a deflection of $7^{\circ}$ removes neutral particles (i.e. neutralization due to interaction with electrons) avoiding defects in the grown films due to $30 \mathrm{keV}$ particles. Before deposition the beam quality as well as the mass resolution of every section of the beam line can be monitored using a Faraday-cup. In the final section of the setup, the ion-beam is swept across the substrate by an electrostatic sweeping system leading to a homogeneous deposition of the thin-film material. In order to suppress neutralization of ions inside the deposition chamber due to interactions with secondary electrons, the setup is equipped with an additional electron lens. Right before deposition, the ion beam is defocused by an electrostatic lens leading to an increased deposition area.

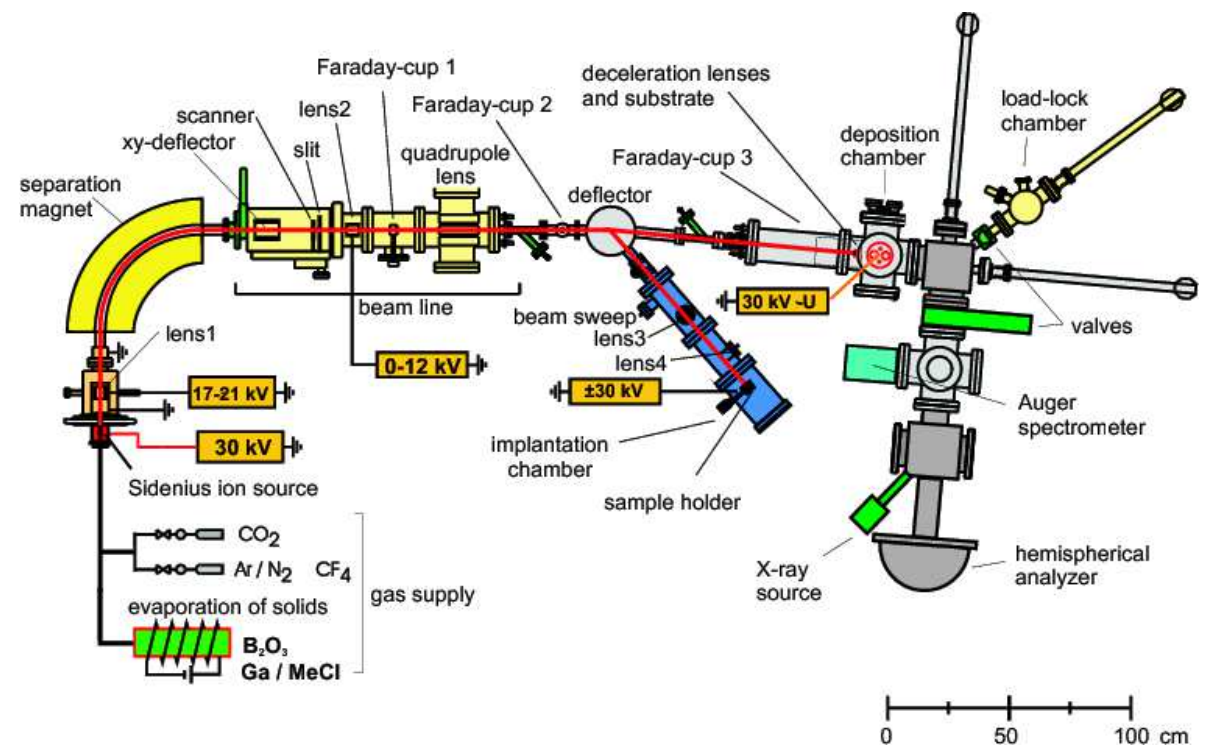

Figure 2.15: Detailed schematic of the ion-accelerator ADONIS (adapted from [11]]). 


\subsubsection{Deposition of ta-C and t-BN thin films}

Prior to deposition, all substrates were cleaned with acetone in an ultrasonic bath followed by a 2-propanol bath or a flush. At last, the liquid film was rapidly removed by a pressurized nitrogen flow. In this way, possible contaminants and dirt are removed from the semiconductor surface. During the process, it is imperative that the substrates stay moistened by the liquid film until all solvents are removed.

In the next step, all samples were in-situ sputter cleaned inside ADONIS using an $1 \mathrm{keV} \mathrm{Ar}^{+}$ion-beam with a total charge of $15 \mathrm{mC}$ in order to remove impurities as well as existing oxide layers from the substrate surface. Furthermore, the surface roughness is reduced inside the surface layer of the substrates. In the growth process of turbostratic boron nitride $(\mathrm{t}-\mathrm{BN}),{ }^{11} \mathrm{~B}^{+}$and ${ }^{14} \mathrm{~N}^{+}$ions were alternately deposited onto the different substrates with an ion energy of $100 \mathrm{eV}$ at a pressure of $10^{-8} \mathrm{mbar}$. In addition, ${ }^{12} \mathrm{C}^{+}$ions were continuously deposited with an ion energy of $100 \mathrm{eV}$ during growth of tetrahedral-amorphous carbon (ta-C) under the same conditions. Between the alternate deposition cycles of $\mathrm{B}$ and $\mathrm{N}$ ions the deposition was stopped for $0.5 \mathrm{~s}$ avoiding possible carbon contamination during switching of the magnet. In comparison to previous work [107], the length of each deposition cycle has been increased from $1 \cdot 10^{15}$ ions/cycle to $4.5 \cdot 10^{15}$ ions/cycle in order to optimize the duration time of the deposition. The stability of the stoichiometry has been verified by additional in-situ characterization of the samples (see sections 4.1.1 and 5.2.2). The growth process of t-BN and ta-C thin-films during MSIBD is well described by the cylindrical spike model by Hofsäss et al. [112]. During deposition, the charge of the ions is measured by a current integrator with an accuracy of a few percent.

The thickness $d_{\text {sample }}$ of each sample can be estimated by measuring the area of irradiation A as well as using the known mass-density $\rho$ of the thin film material, its molar mass $\mathrm{M}$ and the collected charge of the ions q:

$$
d_{\text {sample }}=\frac{q}{q_{e}} \cdot N_{a} \cdot \frac{M}{\rho \cdot A}[m],
$$

with an elementary charge of $q_{e}=1.602 \cdot 10^{-19} \mathrm{C}$. The collected charge of the ions is integrated over time using a current integrator $(\mathrm{CI})^{1}$ and monitored with a PC. In addition, the switching of the magnet as well as the deflection of the beam are automated processes controlled by the same PC.

\footnotetext{
${ }^{1}$ The accuracy of the CI has been verified empirically (see section 5.3 ).
} 


\subsection{Metal top contacts}

In the final step of the sample preparation the MASS diode structure is completed by evaporating metal contacts on top of the grown amorphous semiconductor (AS) thin-film.

These metal contacts were made up of $100 \mathrm{~nm}$ thick pure gold (Au) films as well as of chromium/gold combination (Cr/Au, $10 \mathrm{~nm} / 100 \mathrm{~nm}$ thickness). The $\mathrm{Cr}$ was added to the contact structure in order to increase the adhesion of the Au contacts on the deposited AS thin-films which showed delamination effects in early experiments of this work.

The setup is equipped with two different evaporation sources, more precisely an evaporation boat made up of tungsten and an electron-beam evaporation setup. The two sources are displaced by an angle of $90^{\circ}$. The sample was mounted on a turnable plate and covered by a shadow mask with circular holes of different diameters between $0.6 \mathrm{~mm}$ and $1.1 \mathrm{~mm}$. The shadow mask was made up of copper and is exemplarily shown in figure 2.16 for $0.9 \mathrm{~mm}$ contacts. In order to avoid over-

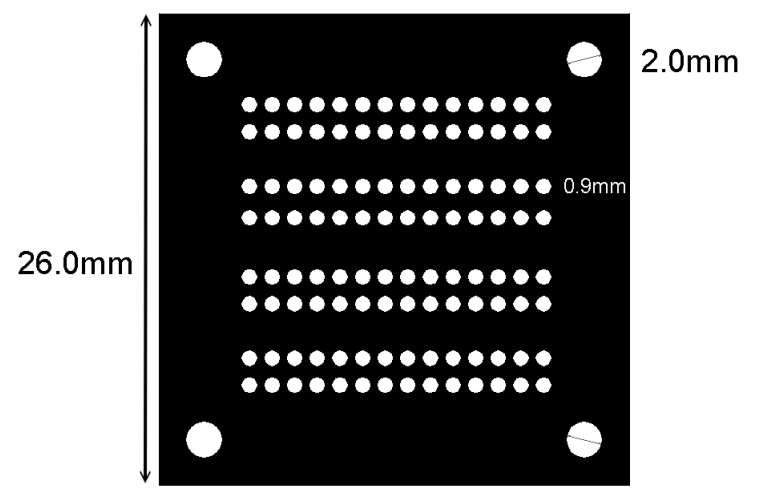

Figure 2.16: Typical shadow mask used for the evaporation of $\mathrm{Cr} / \mathrm{Au}$ top-contacts of the MASS structures. The diameter of circular holes in the example at hand is $0.9 \mathrm{~mm}$.

lapping displacement of the $\mathrm{Cr}$ and $\mathrm{Au}$ films during deposition, the mask has to be close-fitting to the sample. In addition, all samples were mounted at the lowest possible angle regarding both evaporation sources. The thickness of the metal films can be measured using an oscillating crystal at a known density and Z-ratio of the evaporated metal. The crystal is on the same height as the sample plate and is positioned right above the tungsten boat.

Prior to deposition, the chamber was pumped down until a vacuum of $4.0 \cdot 10^{-6} \mathrm{mbar}$, at most, was reached in order to avoid oxidation and contamination effects. During deposition, the chromium is thermally evaporated using the electron beam evaporation setup. As the source material chromium flakes with a purity of $99.9 \%$ were used. Right before the actual deposition some nanometers of the chromium were evaporated in order to get rid of any chromium-oxide contaminants in the raw material surface. During the deposition of the $10 \mathrm{~nm}$ Cr-layer, the pressure was in the 
range of $(0.5-1.0) \cdot 10^{-5}$ mbar. The evaporation rate was measured to approximately (0.4-0.8) $\AA / \mathrm{s}$. Due to the displacement between the e-beam evaporation unit and the crystal there is an error margin of around $30 \%$ regarding these values. After a short cool-down time of the Cr source, the gold is evaporated using the tungsten boat. It is heated by a high DC current power supply leading to evaporation rates of $(2.0-3.5) \AA / \mathrm{s}$ at pressures of $(0.5-1.0) \cdot 10^{-5}$ mbar. 


\section{Chapter 3}

\section{Electrical properties of MASS diodes}

A significant part of this thesis was the investigation of the electrical properties of different MASS diode heterostructures. Regarding the electrical properties of grown thin films during this work, extensive reviews have been given by Jonscher as well as by Simmons on the electronic properties of various dielectric films [113, 114]. The publication of Jonscher mainly discusses the electric properties of amorphous semiconductors while Simmons focused on the properties of insulating films. As disordered or amorphous materials like tetrahedral amorphous carbon (ta-C) or turbostratic boron-nitride (t-BN) show similarities to both classes of materials (see sections 2.4.1 and 2.4.2), basic conduction mechanisms of both have to be considered in this work.

Therefore, the following sections will provide an overview on the basic conduction mechanisms occurring in amorphous and disordered dielectric films. Furthermore, the formation of different heterojunctions will be explained in principle.

\subsection{Ohmic conduction}

Ohmic conduction typically occurs in metals and some semiconductors with low activation energies. The conduction in metals is described by the Drude-model using a free electron gas approximation [115]. The resistance of the metal is caused by interactions of the electrons with phonons and impurity atoms. The corresponding cross-section is a function of temperature and therefore, the resistivity of a metal is also temperature dependent. At very low temperatures near $0 \mathrm{~K}$ the number of interactions is minimal but increases with temperature. Therefore, the resistivity of the metal increases accordingly.

In the process, current-voltage (I-V) characteristics are linear according to Ohm's law [116]:

$$
I=\frac{U}{R}
$$


where $\mathrm{R}$ is the resistance of the conductor. In a simple approximation the resistance can also be calculated by using the cross section area $\mathrm{A}$ of the conductor, its length 1 as well as the specific resistivity of the investigated material $\rho$,

$$
R=\frac{A}{\rho \cdot l}
$$

Regarding the incorporated materials of the investigated heterostructures in this work, an ohmic contact at a metal-semiconductor or metal-insulator interface can only be realized if the work function $\psi$ of the metal is lower than that of the insulator or semiconductor. Otherwise, a Schottky-contact is formed (see section 3.6). The whole process is sketched in figure 3.1. At thermal equilibrium conditions, electrons are injected from the metal into the semiconductor or insulator leading to the formation of a space-charge region and a corresponding induced electric field. In the process, charge carriers accumulate near the interface inside the semiconductor or insulator supplying them according to bias conditions. Therefore, the conduction

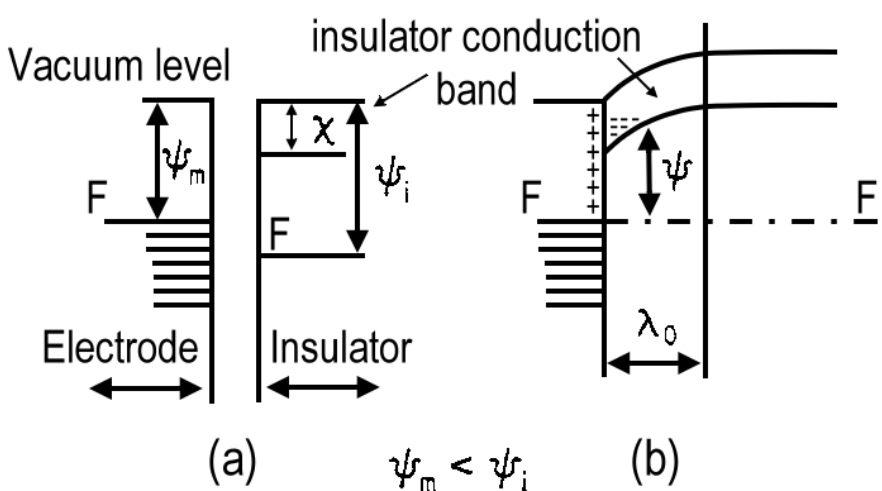

Figure 3.1: Energy diagram of an ohmic contact at a metal-semiconductor or metal-insulator interface (redrawn from [114]). The ohmic contact can only be realized if the work function $\psi$ of the metal is lower than that of the insulator or semiconductor. At thermal equilibrium conditions, electrons are injected from the metal into the semiconductor or insulator leading to the formation of a space-charge region and a corresponding induced electric field. In the process, charge carriers accumulate near the interface inside the semiconductor or insulator supplying them according to bias conditions.

process is limited by the flow rate of electrons inside the bulk of the material (bulklimited).

In a semiconductor, the conductivity is directly proportional to the number of charge carriers and therefore to $\exp \left(-\Delta E /\left(k_{B} T\right)\right)$ with an activation energy $\mathrm{E}$ and the Boltzmann constant $k_{B}$. At higher temperatures and low activation energies this leads to:

$$
I \propto V \cdot \exp \left(\frac{-\Delta E}{k_{B} T}\right)
$$




\subsection{Hopping mechanisms}

In general, amorphous or highly disordered materials often exhibit a form of hopping conduction as part of their electrical properties. In comparison to the forbidden gap of a crystalline semiconductor, amorphous or highly disordered materials possess a high quantity of localized levels in this region. This is exemplarily sketched in figure 3.2. At a very high density of these states, this leads to an overlap of the
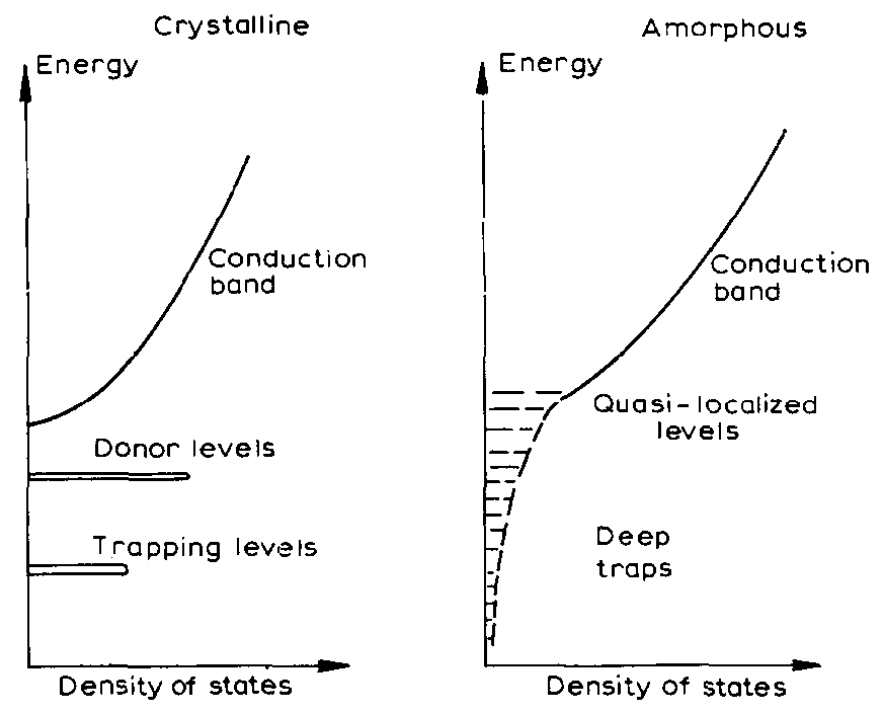

Figure 3.2: Schematic comparison of the electronic band structure of crystalline and amorphous dielectrics (taken from reference [113], (C)1967 Elsevier). In contrast to the forbidden gap of a crystalline semiconductor (left), amorphous or highly disordered materials possess a high quantity of localized levels in that region (right).

corresponding wave functions and an almost metallic conduction.

At greater distances between adjacent centers, electrons can propagate through the material using a phonon-assisted tunneling process from one center to another, the so called hopping conduction. The effect is accompanied by a low mobility of charge carriers and a linear dependence of current and voltage.

Furthermore, the activation energy of such a hopping mechanism is much lower compared to those of donor or acceptor centers in doped semiconductors. However, in non-crystalline materials the activation energy is not clearly defined due to a broad spectrum of deep states in this region [113]. The hopping process itself is provided by a certain percentage of energy from lattice vibrations, e.g. to compensate for the slight difference between energy levels of initial and final state resulting from applied electric fields. Therefore, it also exhibits a slight temperature dependence. 


\subsubsection{Variable Range Hopping}

A more detailed approach regarding the temperature dependence in hopping mechanisms is given by Mott in the Variable Range Hopping (VRH) model [117]. Regarding the hopping process in localized states near the Fermi level based on the thermal activation of electrons, it is dominated by the Boltzmann factor $\exp \left(\frac{E}{k_{B} T}\right)$, with $\mathrm{E}$ being the energy difference between the hopping states, and the phonon frequency of the underlying spectrum. Furthermore, the overlap of the wave-functions regarding adjacent deep-levels have also taken into account. As a result, the conductivity in three dimensions has a form of [118]:

$$
\sigma \propto \sigma_{0} \exp \left(\frac{1}{T^{1 / 4}}\right)
$$

The resulting current-voltage characteristics are linear and comparable to ohmic conduction. Therefore, the two mechanisms can only be distinguished by performing temperature dependent measurements. At higher electrical fields, the characteristics deviate from a linear dependence. As a result, a moderate field correction has to be applied leading to [119]

$$
\sigma \propto \sigma_{0} \cdot \exp \left(\frac{1}{T^{1 / 4}}+\frac{\beta}{k_{B} T}\right)
$$

where $\beta$ is the hopping range of the electrons. In this formula, the physical size of defects, the defect density near the Fermi-level as well as the typical phonon frequency have also been considered. In addition, the conductivity of materials with lower dimensions, e.g. thin-films, can be described by a modified form of equation 3.4 [117]:

$$
\sigma \propto \sigma_{0}(d) \cdot \exp \left(\frac{1}{T^{\frac{1}{d+1}}}\right)
$$

with dimension $d$ and a dimension dependent $\sigma_{0}$ as the volume as well as ranges of possible hopping states depend on the number of available dimensions of the investigated material. At higher temperatures as well as at low barrier heights $\phi$, the conductivity is dominated by electrons excited into the conduction band of the disordered material. In this context, the temperature dependence is given by:

$$
\sigma \propto \exp \left(\frac{\phi}{k_{B} T}\right)
$$

where $\phi$ is $\mathrm{E}_{\text {conduction }}-\mathrm{E}_{\text {Fermi }}$. 


\subsection{Frenkel-Poole effect}

The Frenkel-Poole effect was first reported by J. Frenkel in 1938 [59] and is a a common conduction mechanism occurring in amorphous or disordered semiconducting and insulating materials. It is governed by thermal activation of electrons trapped in localized bound states originating from defects and fixed trap centers between the valence and the conduction band near the Fermi-energy. The average potential of these states is $-\Phi_{\mathrm{FP}}[\mathrm{V}]$ below the conduction band [32].

In principle, the effect is based on a point defect as a center of a Coulomb potential. By applying an electric field of sufficient strength, the barrier height is lowered due to slanted bands, an asymmetric displacement of edges of the barrier. As a result, bound electrons with an electronic energy level of $-\Phi_{\mathrm{FP}}$ are more likely excited into the conduction band and the thermionic emission is enhanced. In this context, the Frenkel-Poole emission process is sketched in figure 3.3. The current through a

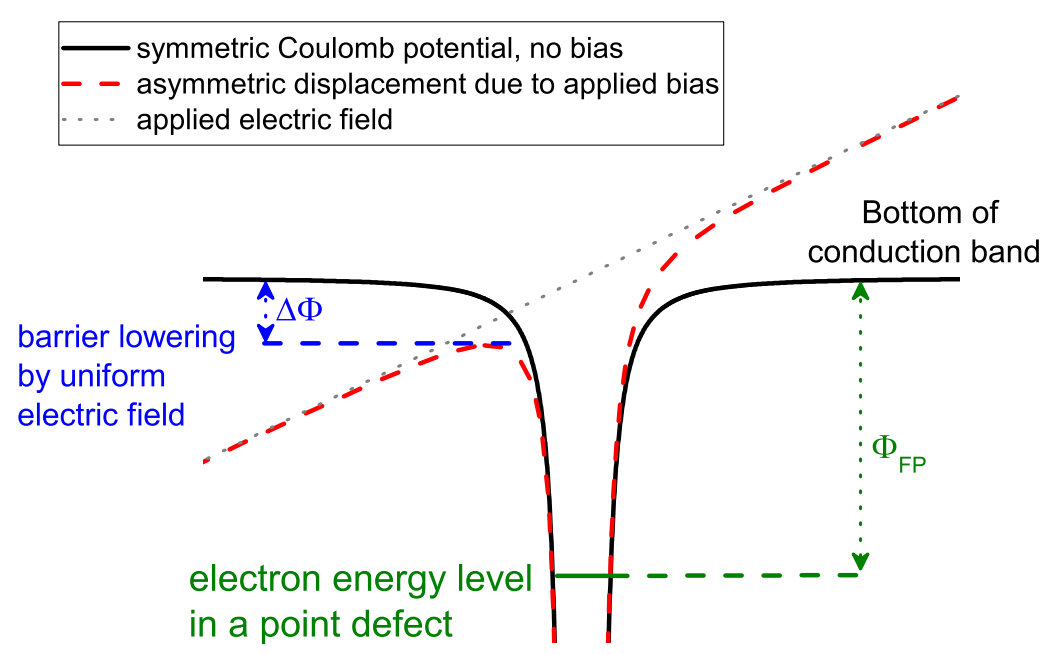

Figure 3.3: The Frenkel-Poole emission process. By applying an electric field of sufficient strength, the barrier height of a Coulomb potential surrounding a point defect is lowered due to slanted bands, an asymmetric displacement of edges of the barrier. As a result, the thermionic emission of electrons is enhanced.

disordered thin-film of thickness $d$ with relative static permittivity $\epsilon_{r}$ and localized states bound at an average barrier height $\Phi_{\mathrm{FP}}$ is given by

$$
I \propto A \sigma_{0} \frac{V}{d} \exp \left[-\frac{q}{k T}\left(\Phi_{\mathrm{FP}}-\sqrt{\frac{q V}{\pi \epsilon_{0} \epsilon_{r} d}}\right)\right] .
$$

Here, a constant electric field $E=V / d$ is assumed across the film, $A$ is the contact area and $\sigma_{0}$ is the electrical conductivity of the thin-film. The I-V charac- 
teristic of a Frenkel-Poole emission dominated sample is typically symmetric and S-shaped. At high electric fields, an almost linear dependence is expected when plotting $\ln (I)$ vs. $\sqrt{V}$, a so called Schottky-plot. Both display formats are exemplarily illustrated in figure 3.4 .

As only point defects in a uniform electric field and therefore emission of electrons into only one direction are covered by the Frenkel-Poole model, it is just a simplified and idealized model. For an application in three dimensions, a perfect symmetrical and spherical shaped potential has to be assumed. Furthermore, a constant barrier height and a fixed dielectric constant inside the volume are assumed for this bulkeffect. As a result, when compared to real current-voltage characteristics based on measured data, equation 3.8 only provides basic information about the conduction mechanism itself, a comparison of theoretical and actual temperature dependence as well as an approximation of the parameters within the formula.
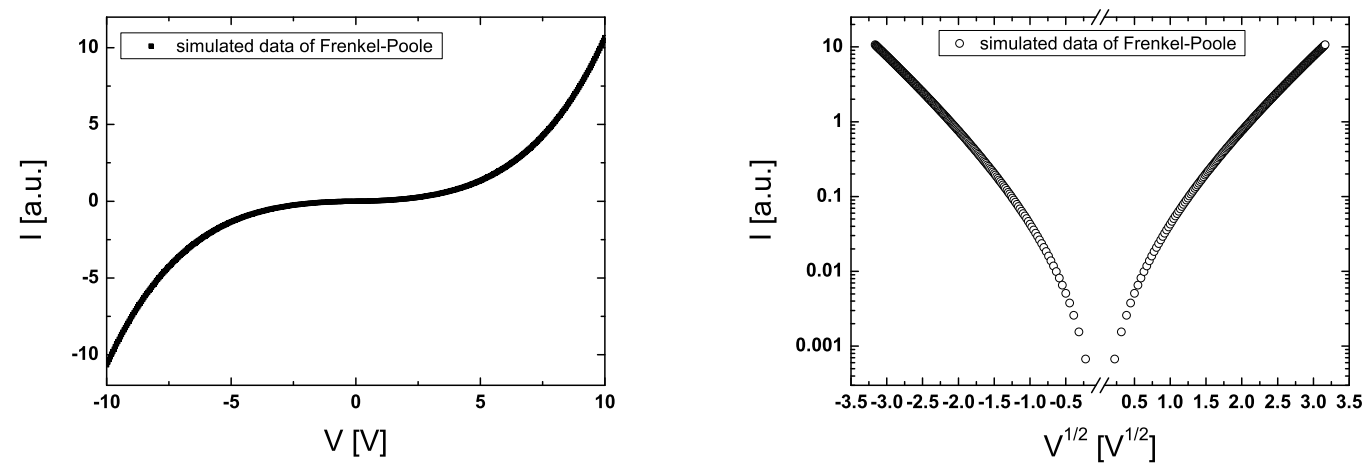

Figure 3.4: Simulated current-voltage characteristic of a Frenkel-Poole resistor according to equation 3.8 on a linear (left) as well as on a logarithmic scale plotted vs. $\sqrt{V}$ (right). In this so called Schottky-plot, an almost linear dependence is expected at high electric fields.

\subsection{Schottky effect}

The Schottky effect is the analogue of the Frenkel-Poole effect at an interfacial barrier. More specific, the Schottky effect, also known as Schottky-Emission, is the thermionic emission of charge carriers over an interface barrier between a metal and a dielectric lowered by an external electric field. In this context, the lowering of the potential energy is induced by the corresponding image force. The process is displayed in figure 3.5. The corresponding current-voltage characteristic can be described by the Richardson-Schottky-equation:

$$
I \propto A^{*} T^{2} \exp \left[\frac{-q}{k T}\left(\Phi_{\mathrm{SE}}-\sqrt{\frac{q V}{4 \pi \epsilon_{0} \epsilon_{r} d}}\right)\right],
$$

where $A^{*}$ is the effective Richardson constant and $\Phi_{\mathrm{SE}}$ the barrier height of the Schottky-emission. All other parameters have already been described in equation 


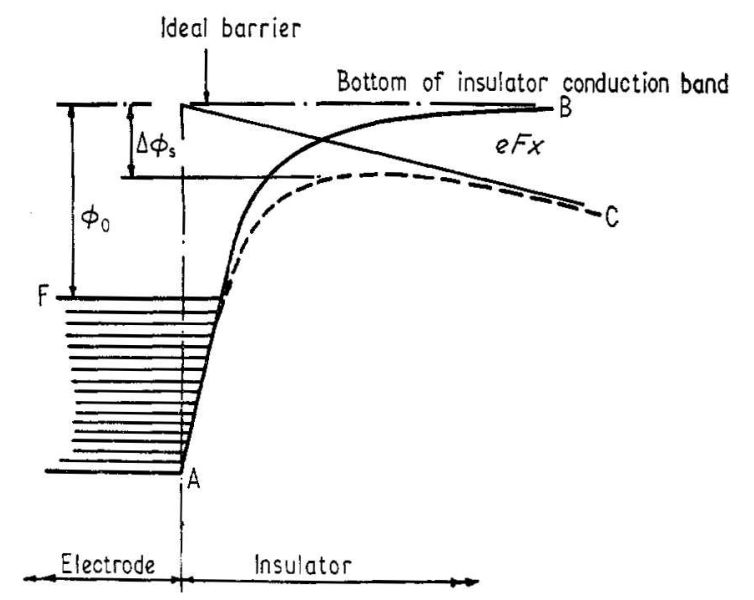

Figure 3.5: Schottky effect at an interfacial barrier (taken from 114]). The effect is based on the thermionic emission of charge carriers over interface barrier between a metal and a dielectric lowered by an external electric field $e F$. In this context, the lowering of the potential energy is induced by the corresponding image force.

3.8. At high electric fields, the corresponding characteristic is quite similar to that of Frenkel-Poole conduction (see figure 3.6). The formula also leads to a linear

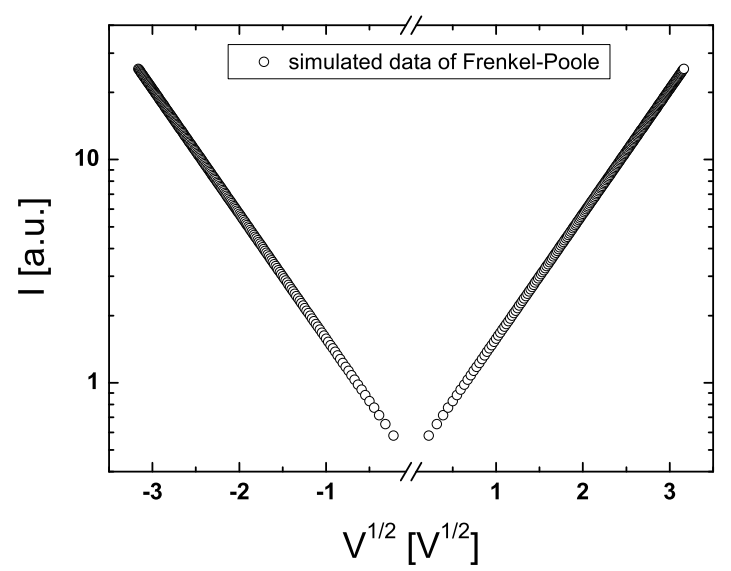

Figure 3.6: Simulated current-voltage characteristic of a Schottky resistor according to equation 3.9. Drawn in a Schottky-plot, the characteristic is linear.

characteristic at high electric fields in a Schottky-plot.

However, the temperature dependence of Schottky Emission differs from the FrenkelPoole effect due to the additional $T^{2}$ as well as the characteristic at low electric fields resulting from the missing linear component of the electric field. Furthermore, the additional factor of 4 in the equation originates from a different geometry as the distance between the electron and its mobile image charge is two times the distance between electron and local traps regarding the FP-effect. 


\section{5 p-n junction diode - the Shockley model}

In principle, a p-n junction is formed at the interface between a p-type and a n-type doped semiconductor. If the junction consists of only one material, it is called a homojunction, otherwise a heterojunction. The formation is explained in principle on the basis of a homojunction for simplicity reasons and displayed in figure 3.7. In a theoretical, decoupled state, the Fermi-levels of the semiconductors differ by

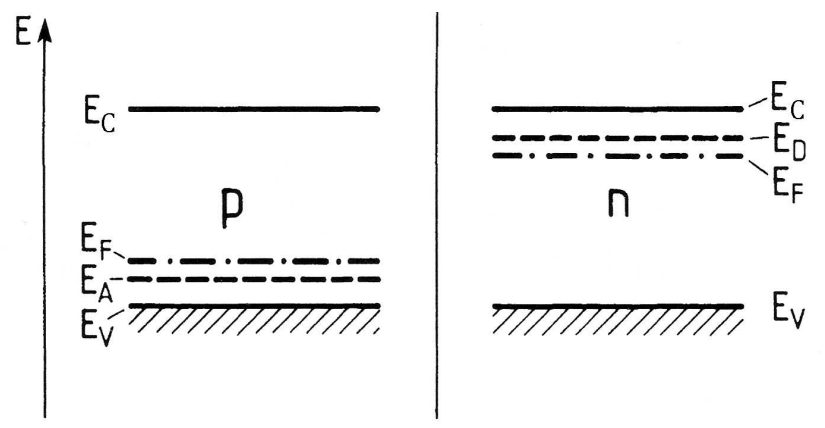

Figure 3.7: Band-scheme of a pn-diode based on a homojunction in a theoretical, decoupled state (adapted from [120]). The Fermi-levels of the semiconductors differ by an energy difference based on their particular acceptor and donor concentrations and levels.

an energy difference based on their particular acceptor and donor concentrations and levels. In a "real" crystal under thermal equilibrium conditions the Fermienergy has to be constant resulting in band bending, shown in figure 3.8. At the

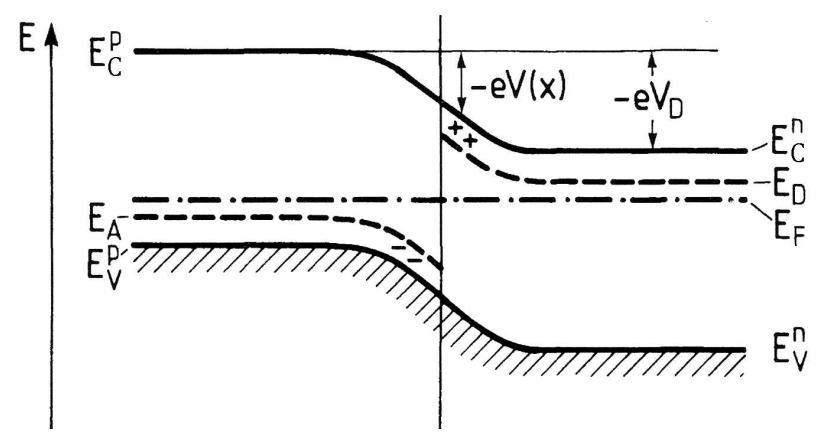

Figure 3.8: Band-scheme of a pn-diode based on a homojunction in a coupled state (adapted from [120] ). In a "real" crystal under thermal equilibrium conditions the Fermi-energy has to be constant resulting in band bending.

same time, regarding the interface region of the $\mathrm{p}-\mathrm{n}$ junction, there is a gradient in concentration of opposite charge carriers (electrons and holes). According to the law of mass action, the product of concentrations related to electrons (n) and holes (p) has to be constant at thermal equilibrium. This can be written as:

$$
n_{i}^{2}=n p
$$


A non-equilibrium state leads to diffusion of electrons into the p-type doped region of the junction and holes accordingly towards the n-type doped semiconductor. The resulting ionized states of the donor and acceptor atoms near the interface of the junction lead to the formation of an electric field which, on the other hand, counters the continuous diffusion of free charge-carriers without an external field. In this state, thermal equilibrium is reached and the so called space-charge- or depletionregion is formed. The whole process is sketched in figure 3.9. Furthermore, the band

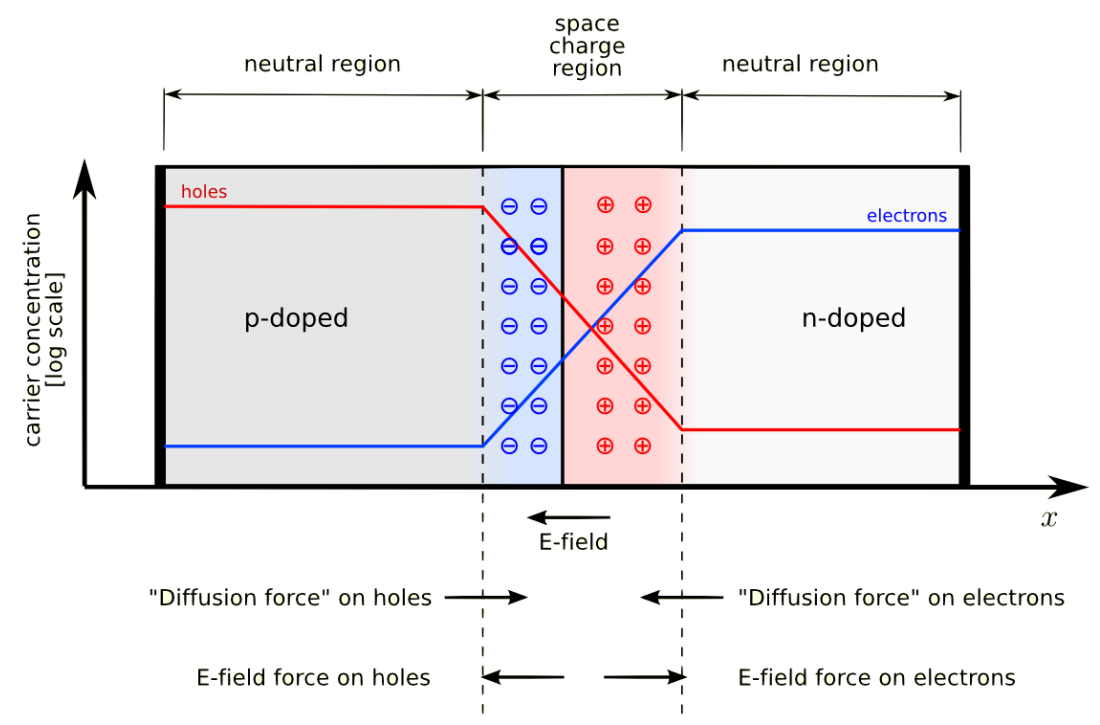

Figure 3.9: Formation of a space-charge region at thermal equilibrium conditions (from [121]). Regarding the interface region of a "real" $p$-n junction, there is a gradient in concentration of opposite charge carriers (electrons and holes). Such a non-equilibrium state results in diffusion of electrons into the p-type doped region of the junction and holes accordingly towards the n-type doped semiconductor. The resulting ionized states near the interface lead to the formation of an electric field which counters the continuous diffusion of free charge-carriers without an external field. As a result, thermal equilibrium is reached and the so called space-charge- or depletion-region is formed.

bending can be approximated by a so called macro potential $\mathrm{V}(\mathrm{x})$ at position $\mathrm{x}$ of the junction and linked to the corresponding space-charge $\rho(x)$ using the Poisson equation:

$$
\frac{\partial^{2} V(x)}{\partial x^{2}}=\frac{\rho(x)}{\epsilon \epsilon_{0}}
$$

If the diode is biased by an external potential $\mathrm{V}$, the band edges as well as the Fermi-levels will be displaced against each other by a value of e| $V \mid$, resulting in a variation of the extent of the space-charge-region depending on the polarity of the potential with respect to the diode. Based on the processes described above, a reduction of barrier height leads to a shrinkage of the space-charge-region and an increase in charge carrier transport (figure 3.10a). The p-n junction is operated at forward-bias direction. By biasing the junction in opposite direction, the width of the space-charge-region increases, the junction is operated at reverse-bias direction 
(a) reverse-bias
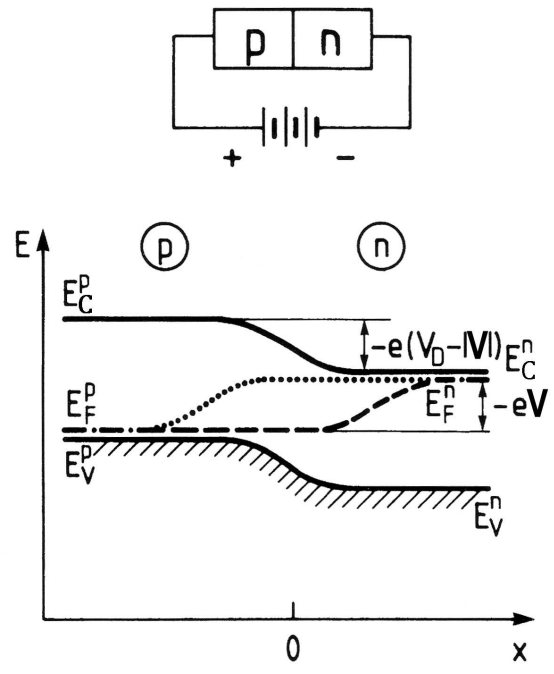

(b) forward-bias
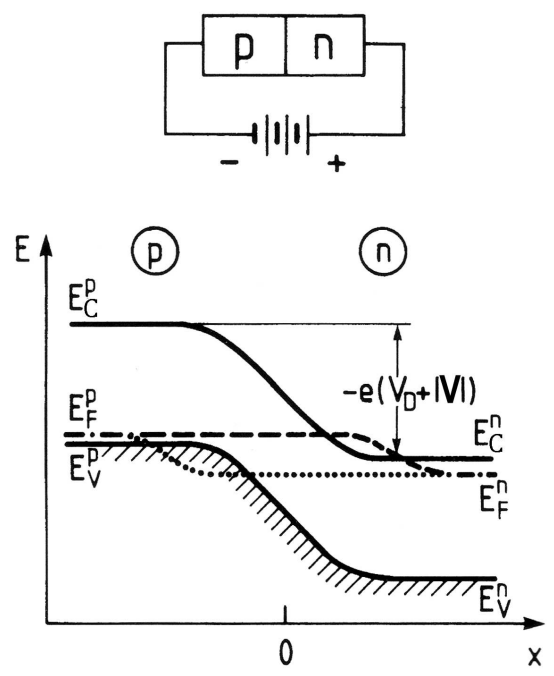

Figure 3.10: Band-scheme of a biased pn-diode based on a homojunction (adapted from [120]). An external potential $\mathrm{V}$ causes a displacement of the band edges as well as the Fermi-levels by a value of e $|V|$, resulting in a variation in dimension of the space-charge-region depending on the polarity of the potential with respect to the diode. (a) A reduction of barrier height leads to a shrinking of the space-charge-region and an increase in charge carrier transport. The p-n junction is operated in forward direction. (b) By biasing the junction in opposite direction, the width of the space-charge-region increases, the junction is operated at reverse-bias direction.

(figure $3.10 \mathrm{~b}$ ). The corresponding current-voltage characteristic of a $\mathrm{p}-\mathrm{n}$ junction diode can be described by the Shockley equation $([32,33])$ :

$$
I=I_{s} \cdot\left(\exp \left[\frac{q V}{n k T}\right]-1\right)
$$

where $I_{s}$ is the saturation current, $T$ the temperature of the diode and $n$ the so called ideality factor of the diode.

For ideal p-n diodes, in particular, desirable for solar cell application, an ideality factor approaching unity is desired. The ideality factors of real $\mathrm{p}-\mathrm{n}$ diodes may be described by the Sah-Noyce-Shockley theory, published in 1957 [28], yielding values of $n=1$ at a low voltage and $n=2$ at higher voltages. If there were no defects present, the total diode current would be based exclusively on diffusion and $n$ would be 1 . Therefore, no recombination processes are expected inside the spacecharge region. The resulting minimization of leakage current loss at reverse bias and increased rectification characteristic are favorable properties of such devices. As recombination processes are driven by defects, more defects lead to more spacecharge recombination increasing $n$ up to 2 (the "non-ideal" case). For p-n diodes, an ideality factor $n>2$ is not covered by the Sah-Noyce-Shockley theory and suggests the presence of surface or interface states, indicating that the junction is far from being ideal. 


\subsection{Schottky diode}

By evaporating a metal contact onto the surface of a semiconducting or almost insulating material, a Schottky contact is formed at the interface. The corresponding junction, a Schottky diode, exhibits very similar properties compared to a p-n junction diode. In principle, one side of the $\mathrm{p}-\mathrm{n}$ junction is replaced by a metal contact. Hence, a Schottky-contact is often referred to as "one-sided p-n junction". Regarding the interface conditions of the junction, the band structure of the semiconductor is "pinned" to states at the metal-semiconductor interface and the Fermi-levels have to be equal at thermal equilibrium conditions. Therefore, the band structure of the semiconductor is bend. If the semiconductor is n-type doped, the donors in the interface region will be depleted and the electrons diffuse in the direction of the metal. As a result, a depletion region is formed inside the semiconductor due to the positive charge while the negative charge accumulates inside the metal contact. The band-scheme of such a Schottky diode is shown in figure 3.11. If, on the other hand,

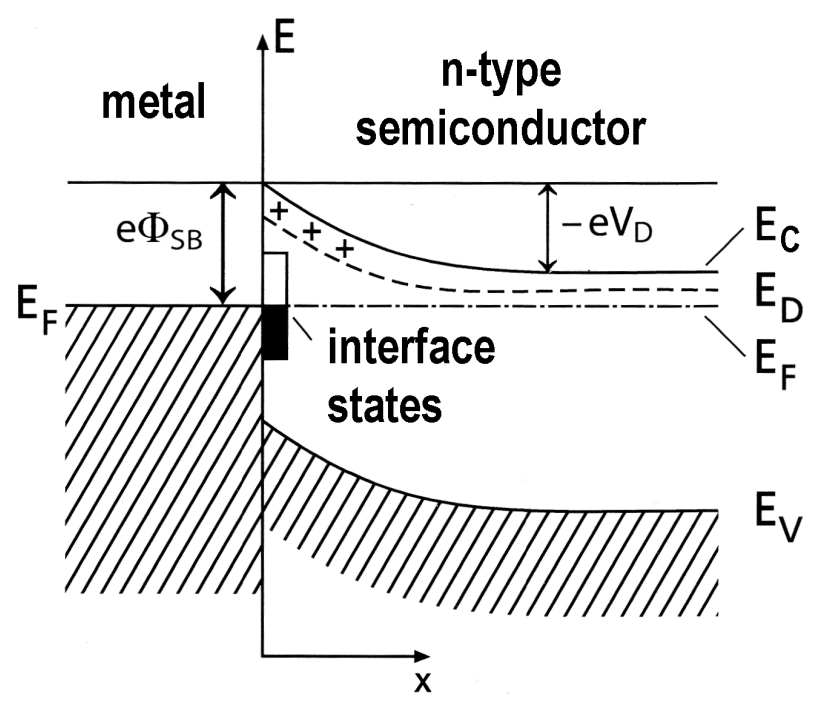

Figure 3.11: Band-scheme of a Schottky diode based on a metal/n-type semiconductor junction (adapted from [120]). The band structure of the semiconductor is "pinned" to states at the metalsemiconductor interface and the Fermi-levels have to be equal at thermal equilibrium conditions. Therefore, the band structure of the semiconductor is bend. The donors in the interface region are depleted and the electrons diffuse in the direction of the metal. As a result, a depletion region is formed inside the semiconductor due to the positive charge while the negative charge accumulates inside the metal contact.

the semiconductor is p-type doped, electrons will diffuse from the metal into the semiconductor ionizing the acceptor states inside the interface region. Therefore, the holes of the semiconductor are depleted and the space-charge-region is formed accordingly.

The effects of an external potential are comparable to those described for $p-n$ junctions above, but only one side of the junction, the semiconductor, is affected. At 
forward bias, the potential barrier is lowered and the charge carrier transport is increased. At reverse bias, only a few electrons can overcome the barrier leading to very low currents. However, there is also a probability to tunnel through the barrier. A corresponding sketch is shown in figure 3.12, The tunneling current $\mathrm{T}(\mathrm{E})$

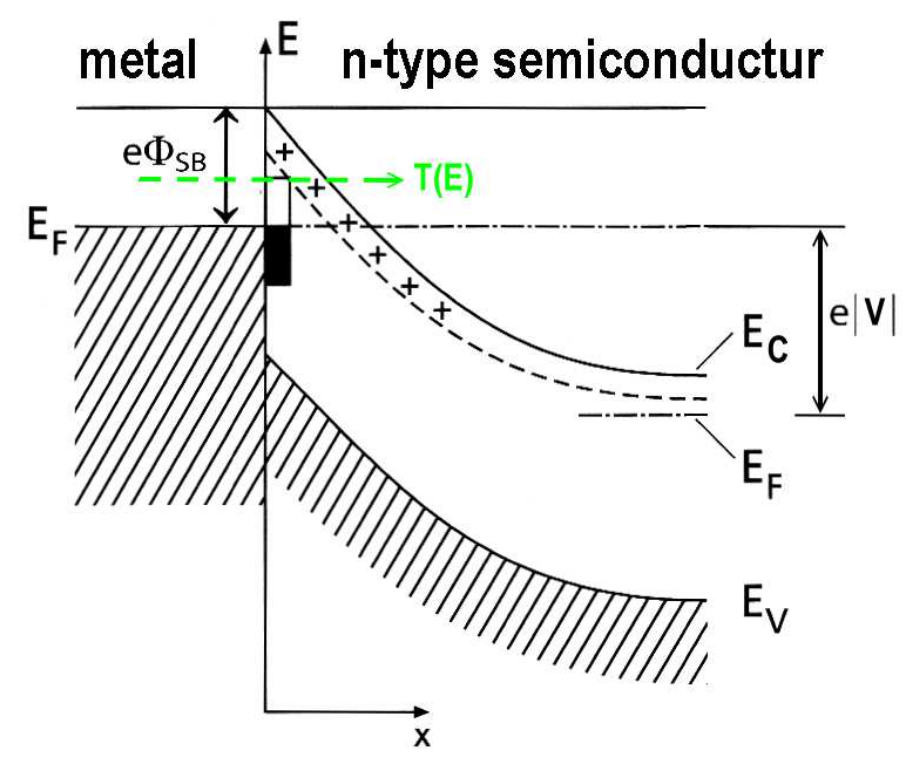

Figure 3.12: Tunneling principle in a reverse-biased Schottky diode based on a metal/n-type semiconductor junction (adapted from [120]). At reverse bias, only a few electrons can overcome the barrier leading to very low currents. However, there is also a probability to tunnel through the barrier. The tunneling current $\mathrm{T}(\mathrm{E})$ increases with higher bias-voltages and corresponding field-intensity $\mathrm{E}$ due to deformation of the barrier leading to an increase in height and a significant reduction in width.

increases with higher bias-voltages and corresponding field-intensity E due to deformation of the barrier leading to an increase in height and a significant reduction in width. Finally, after reaching a certain current density, such a junction breaks down and is irreversibly damaged as impurity atoms might form a conducting filament along the tunneling path. The current-voltage characteristic of a Schottky diode is very similar to a p-n junction diode and can also be described by the Shockley equation. In this context, reverse saturation current given by [29, 30]

$$
I_{s}=A A^{*} T^{2} \exp \left(-\frac{q \Phi_{s}}{k T}\right)
$$

where $A$ is the diode area, $A^{*}$ is the effective Richardson constant, and $\Phi_{s}$ is the Schottky barrier height of the metal-semiconductor junction. As a result, such heterojunctions exhibit a different temperature dependence when compared to $\mathrm{p}-\mathrm{n}$ junctions. The effective Richardson constant can be calculated using the effective mass $m_{\mathrm{eff}} m_{\mathrm{e}}$ of the majority charge-carriers of the semiconducting material in the following expression:

$$
A^{*}=4 \pi \cdot q \cdot m_{\mathrm{eff}} m_{\mathrm{e}} \cdot \frac{k^{2}}{h^{3}} .
$$


The extraction of Schottky diode parameters from forward current-voltage characteristics is discussed by Cheung et al. providing some helpful suggestions on the analysis of I-V characteristics based on MASS diodes [29].

\subsection{Tunneling mechanisms}

Another conduction mechanism in dielectrics is tunneling. As mentioned in section 3.6. the tunneling mechanism occurs at high but thin barriers. The transmission probability is anti-proportional to the thickness of the barrier and the shape of the barrier has also to be taken into account. In addition, there is also a certain probability for the transmission of an electron trough the semiconductor or insulator based on the thickness of the material.

Corresponding current densities $J$ regarding tunneling through a barrier have been calculated by Simmons et al. [114] on the basis of a trapezoidal shaped barrier using the Wentzel-Kramers-Brillouin (WKB) approximation which considers the limit of extremely thin boxes forming an integral. This derivation is described by many quantum mechanical lecture books, e.g. by Schwabl [122]. In addition, similar calculations were also performed by O'Dwyer [123] on the basis of a triangular potential leading to comparable results.

The calculations by Simmons et al. result in rather complex formulas regarding the thermal, temperature dependent as well as the isothermal J-V characteristic of such a system. As one result, they showed that there is almost no temperature dependence present. As the percentage change in current density is only in the range of around one tenth between $0 \mathrm{~K}$ and room temperature, the effect is rather small. Furthermore, at high electric fields, the calculated equations lead to the so called Fowler-Nordheim equation which can be used as an approximation for the current density of the tunneling process. Considering the effective mass of electrons $m^{*}$ inside the insulator or semiconductor, the current density is given by

$$
J=\alpha E^{2} \exp \left(-\frac{\beta}{E}\right)
$$

with

$$
\begin{gathered}
\alpha=1.5413 \cdot 10^{-6} \sqrt{\frac{m}{m^{*} \Phi}}, \\
\beta=6.828 \cdot 10^{7} \sqrt{\frac{m^{*}}{m}} \Phi^{\frac{3}{2}} .
\end{gathered}
$$

Finally, there are also reports in literature about constant residual conductivity phenomena at low temperatures, which occur in disordered thin-film systems, comparable to those of this thesis. However, they cannot be linked to any thermally activated mechanism like Frenkel-Poole-Emission, Schottky-emission or Variable Range Hopping (see section 3.3, 3.4 and 3.2.1) even if the corresponding characteristics show 
similar behavior in some parts. Several authors suggest, that such an effect originates from some kind of temperature independent tunneling process which cannot be linked to those described above [124, 125].

In order to explain such a characteristic, a tunneling mechanism exhibiting a linear characteristic at high electric fields in a Schottky plot $\left(\ln (\mathrm{I})\right.$ vs. $\mathrm{V}^{1 / 2}$ ), similar to Frenkel-Poole- or Schottky-emission, is described in the following based on the work of O'Dwyer et al. [126]:

Electrons in a dielectric material exhibiting localized states near the conductions band can occasionally be excited even at low temperatures after being exposed to an electric field of high intensity. If the number of interactions between these trapped electrons and the surrounding lattice is much lower than interactions involving states in the conduction band, the temperature of the electrons is likely higher compared to the surrounding lattice. This assumption eventually leads to to a tunneling characteristic similar to Frenkel-Poole or Schottky emission at high electric fields in a Schottky-plot $\left(\ln (\mathrm{I})\right.$ vs $\left.\mathrm{V}^{1 / 2}\right)$, more precisely:

$$
J \propto \frac{V}{d} \exp \left(\frac{E_{g}}{\Delta E} \frac{V^{2}}{\tilde{F}^{2} d^{2}}\right),
$$

where $\mathrm{V}$ is the bias-voltage, $\mathrm{d}$ the layer thickness, $\mathrm{E}_{g}$ the band gap, $\Delta \mathrm{E}$ the range of the shallow traps and $\tilde{F}$ the breakdown field strength.

\subsection{Photoconductivity in semiconductors}

In general, the basic aspect of photoconductivity is the increase of electrical conductivity of semiconductors or insulators after irradiation with electromagnetic waves of a broad spectrum from infrared light to gamma radiation [127 129]. In the process, illustrated in simplified terms in figure 3.13, the light is absorbed by the irradiated material, e.g. a semiconductor as in this thesis. As a result, the number of free electrons and holes changes due to the generation of electron-hole pairs. In an intrinsic semiconductor (1), the corresponding energy $E=h \nu$ has to exceed or at least match the band-gap energy $E_{\text {gap }}$ of the semiconductor in order to generate enough free charge carriers inside the semiconductor by excitation. If the semiconductor is additionally doped, e.g. p-type, there will be acceptor states present in the semiconductor (2), leading to electron-hole pair generation at much lower energy levels of the absorbed electromagnetic waves. However, due to the limited number of acceptor states, the effect on electrical conductivity regarding such an excitation is much lower compared to the irradiation with electromagnetic waves exceeding the energy of the band-gap. In addition, the same arguments are valid for n-type semiconductors exhibiting donor states near the conduction band (3). Therefore, the electrons can be excited out of donor states right into the conduction band leading to electron-hole pair generation at a similar energy range. Finally, the same processes can also be triggered by additional defect states near the valence or the conduction band (marked blue). 
Furthermore, the conductivity of a material can be investigated as a function of photon energy (or rather wavelength of light). This spectral response typically exhibits a rather pronounced maximum near the band-gap energy of the material, the equivalent of the minimal energy required to excite an electron from a bonded state into the conduction band. Furthermore, photoconductivity also occurs in biased semi-

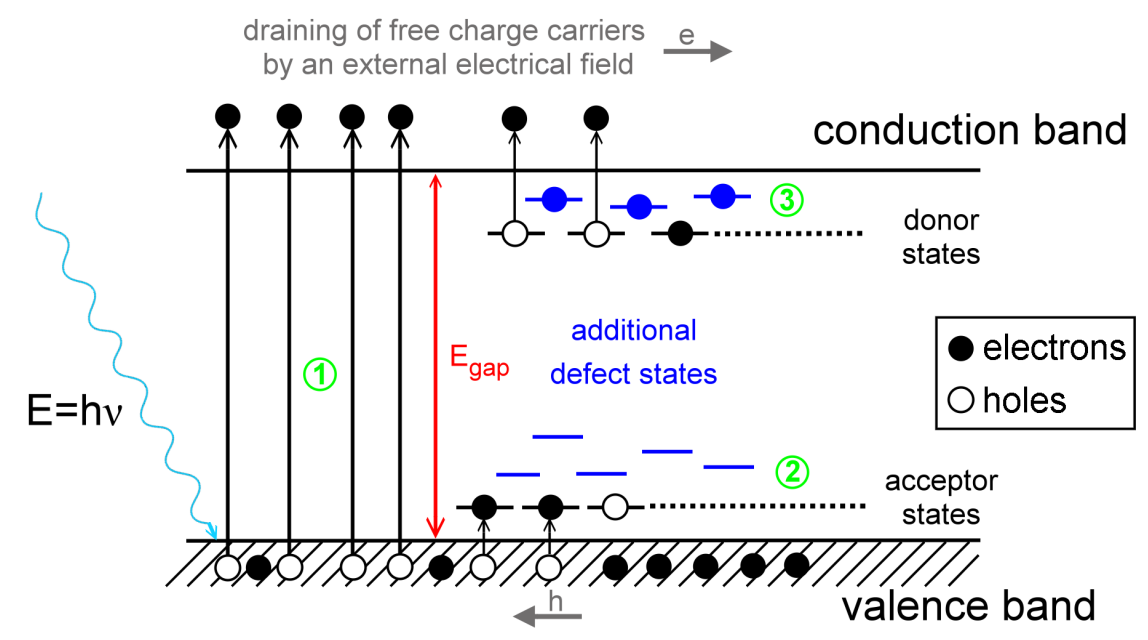

Figure 3.13: Principle of photoconductivity. The increase of electrical conductivity of semiconductors or insulators is basically caused by the generation of electron-hole pairs resulting from irradiation with electromagnetic waves. (1) In an intrinsic semiconductor, the corresponding energy $E=h \nu$ has to exceed or at least match the band-gap energy $E_{g a p}$ of the semiconductor in order to generate enough free charge carriers inside the semiconductor by excitation.

(2) If the semiconductor is p-type doped, there are additional acceptor states present near the valence band. As a result, the level of energy required for electron-hole pair generation significantly lowered.

(3) A similar behavior can be observed for a n-type semiconductor for excitations of electrons into the conduction band. In addition, the same processes can also be triggered by additional defect states near the valence or the conduction band (marked blue).

conductor heterostructures leading to the fabrication of photosensitive devices, e.g. photodiodes. They are usually made up of a p-n junction or a PIN heterostructure. If the incident light is absorbed in the depletion region of the device the generated carriers will be drained by the electrical field inside the space-charge-region towards the electrodes resulting in a photocurrent. When operated at reverse bias, the width of the depletion region increases and the capacitance decreases. As a result, the response times of such devices are drastically reduced.

In addition, another characteristic property of such devices is the decay time of photoconductivity, more precisely the time until equilibrium conditions are reached right after stopping the excitation. It is correlated to the lifetime of charge carriers and the density of potentially trapped charge carriers. As a result, a detailed analysis of the decay time and comparison of different heterostructures might provide some information about the origin of photoresponses as well as the underlying excitation processes. 


\section{Chapter 4}

\section{Characterization techniques}

\subsection{Structural characterization}

The structural characterization of the grown thin-films was performed in-situ by X-ray photoemission spectroscopy (XPS) in order to determine the chemical composition of the grown thin-films as well as the dominating crystallographic phase. In addition, the micro-structure of all fabricated heterostructures has been analyzed by performing cross-section transmission electron microscopy (TEM) measurements.

\subsubsection{X-ray photoemission spectroscopy (XPS)}

The X-ray photoemission spectroscopy (XPS) is an in-situ analysis method of ADONIS (see section 2.5.2) and used to analyze the composition of the grown thin-films directly after the deposition process. The functional principle is based on the photoelectric effect described by Einstein in 1905 [130]. In detail, the surface of the sample is irradiated by a X-ray beam at a photon energy of $h \nu$. As a result, electrons are ejected from occupied states in the inner-shell orbitals of surface atoms with a kinetic energy $E_{k i n}$. Compared to the original excitation energy $h \nu$, the kinetic energy is reduced by the binding energy $E_{b}$ of the exited electrons as well as the work function $\Phi$ of the solid [131]

$$
E_{k i n}=h \nu-E_{b}-\Phi .
$$

This leads to a mapping of occupied electronic states in solids (binding energy $E_{b}$ ) according to their distribution. Therefore, it is possible to analyze the chemical composition as well as the stoichiometry of the thin film surfaces. In addition, XPS provides information about possible plasmon excitations inside the sample surface during irradiation. The plasmon energy can directly be linked to the $\mathrm{sp}^{2}$ - to $\mathrm{sp}^{3}$-ratio of the bonds inside the analyzed solid leading to information about the crystalline structure of the sample. Due to these plasmon excitations, the kinetic energy is reduced by an additional value of $\hbar \omega_{\mathrm{P}}$, where $\omega_{\mathrm{P}}$ is the plasmon frequency

$$
E_{k i n}=h \nu-E_{b}-\Phi-\hbar \omega_{\mathrm{P}}
$$




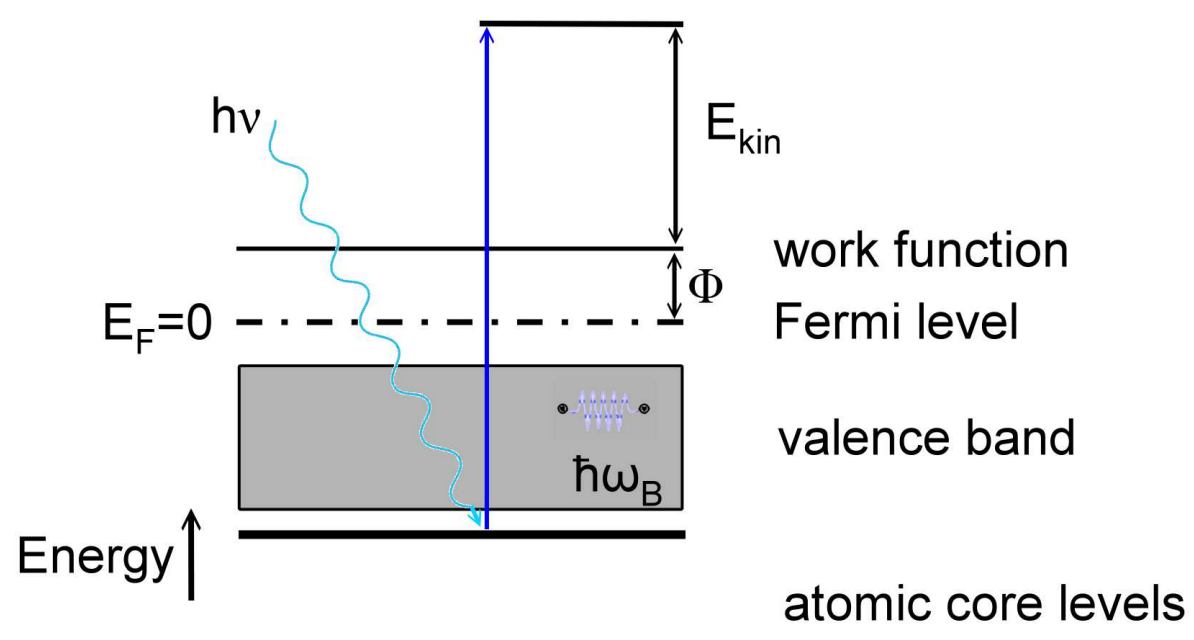

Figure 4.1: Principle of X-ray photoemission spectroscopy (XPS). The surface of the sample is irradiated by a focused X-ray beam at a photon energy of $h \nu$. As a result, electrons are ejected from occupied states in the inner-shell orbitals of the surface atoms with a kinetic energy $E_{k i n}$. Compared to the original excitation energy $h \nu$, the kinetic energy is reduced by the binding energy $E_{b}$ of the exited electrons as well as the work function $\Phi$ of the solid.

All XPS-measurements were performed in-situ on several ta-C as well as t-BN based samples right after deposition at a pressure of $5 \cdot 10^{-9}$ mbar. The excitation was realized using an Omicron DAR400 X-ray source which provides characteristic X-ray lines of $\mathrm{Mg}-K_{\alpha}$ as well as $\mathrm{Al}-K_{\alpha}$.

In this thesis, the $\mathrm{Mg}-K_{\alpha}$ line at an energy of $1253.6 \mathrm{eV}$ and an emission current of $12 \mathrm{~mA}$ were used. The intensity was always measured as a function of binding energy. The photo electrons were detected by an Omicron EA125 hemispherical analyzer. It is equipped with 7 single channel electron multiplier, so called Channeltrons, and powered by an EAC2000-125 power supply. The energy scale of the detector is calibrated prior to the actual XPS-measurements and described in detail in section 5.1. The information depth of the analyzed samples is in the range of just a few nanometers. The exact range depends on the inelastic mean free path of the electrons in the analyzed material. 


\subsubsection{Transmission-Electron-Microscopy (TEM)}

In order to investigate the crystalline micro-structure of the grown samples on the nanometer scale, cross section transmission electron microscopy (TEM) measurements were performed on all fabricated heterostructures.

Each cross section TEM lamella was cut out along the surface normal using a focused ion beam (FIB) setup, more precisely a Dual FIB Nanolab 600 from FEI. The cut out and the subsequent thinning processes down to a few nanometers were performed using a focused Ga-ion-beam at $30 \mathrm{kV}$ acceleration voltage as well as various current settings and resulting sputter rates. The lamella was fixed to a $\mathrm{Cu}$ TEM-grid, also using Pt, as it is exemplarily shown in figure 4.2. Prior to the

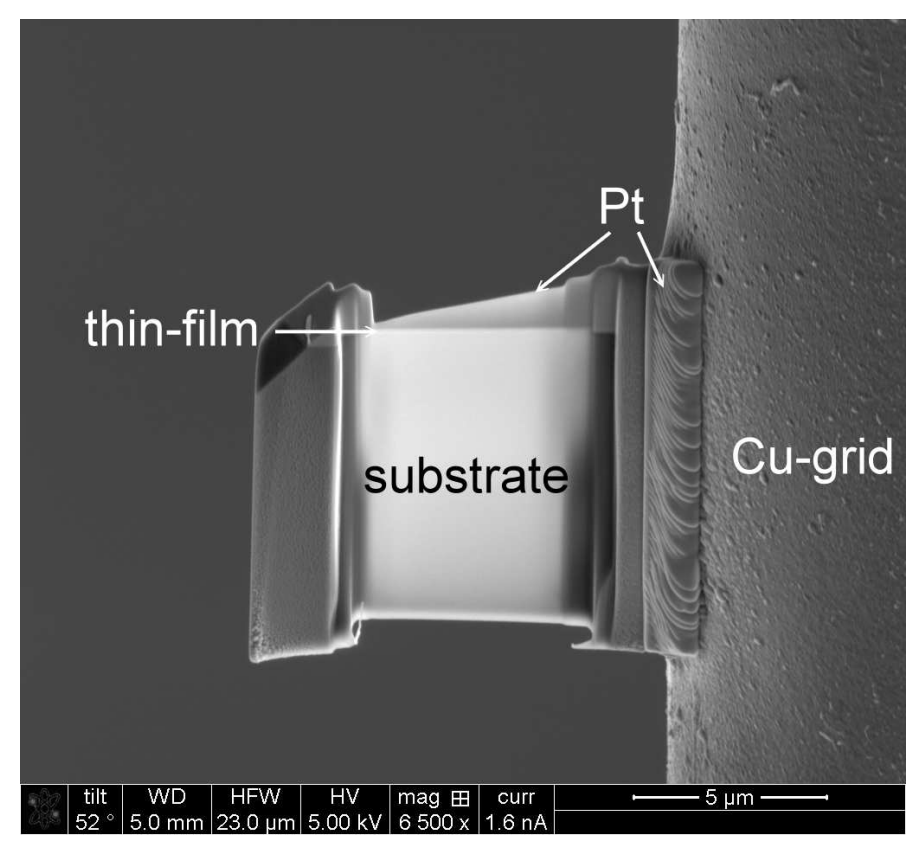

Figure 4.2: Typical SEM-images of cross-section TEM-lamellas right after the focused ion-beam preparation process. The lamellas are fixed to the $\mathrm{Cu}$-grid with platinum.

lamella preparation, the surface of the sample was covered by a thin Pt-layer with dimensions of $12 \times 1.5 \times 0.2 \mu \mathrm{m}^{3}$ protecting the sample's surface against ion beam sputtering. During the whole process, all samples could be examined right away using an integrated scanning electron microscope (SEM). In principle, the whole FIB preparation was performed according to the detailed guideline provided by the PhD-thesis of Dr. H. Zutz [132].

Subsequently, the actual TEM measurements were performed using a Philips CM200-FEG-UT microscope provided by the group of Prof. Dr. M. Seibt at the 4th institute of Physics, University of Göttingen. It is equipped with a field emission gun (FEG) serving as the electron source. These electrons are accelerated by a voltage of $200 \mathrm{kV}$. The resulting wavelength of the electrons combined with an arrangement of several lenses and apertures results in a resolution of this setup almost down to the atomic scale. In theory, the resolution could be even higher according to Abbe's law 
but is limited by quality of the electron lenses used in the experimental setup. In the present experiment, the high resolution lens provides a point resolution of $0.188 \mathrm{~nm}$. The microscope itself, on the other hand, provides a minimal resolution of the electron beam down to about $0.5 \mathrm{~nm}$. The images of TEM- and HRTEM-measurements were recorded using a fiber-optic coupled and peltier cooled CCD-camera setup from Gatan.

The TEM-measurements, provided by this setup, lead to a detailed analysis of thinfilm and possibly present interlayer thicknesses as well as the layered structure of the grown heterostructures, especially the MASS diode structures.

In addition, high resolution TEM-measurements (HRTEM) with magnifications of up to $560 \mathrm{k}$ were performed in order to determine the precise crystallographic orientation of the grown thin-films (e.g. t-BN) as well as the defect density inside the grown thin-films and the surface of the substrates. Furthermore, the roughness of the substrates' surface and possible interface layers were investigated in detail.

In addition, the microscope also provides a scanning transmission electron microscopy (STEM) mode. The focused electron beam is scanned across the sample causing the generation of characteristic X-rays. The corresponding energies can be linked to the excited orbitals of elements inside the TEM-lamella. The X-rays can be analyzed using an energy dispersive X-ray (EDX) setup, more precisely a Link ISIS setup from Oxford Instruments. It has an energy range of 0-20 keV and a resolution of 1024 channels. In this context, it has to be noted that elements with masses lower then carbon cannot be detected by this setup due to noise at the lower end of the energy spectrum caused by the detector itself. Furthermore, the focused electron beam can also be scanned along a line, e.g. perpendicular to the substrate surface, leading to a detailed chemical composition analysis along the scan path including crucial areas like the interface layer of the grown heterostructures.

The results of all these measurements provide possible indications for the actual growth process and will also be linked to the electrical properties of the grown samples in the course of this work (see sections 5.3 and 66). 


\subsection{Electrical characterization (DC)}

The electrical characterization of the grown MASS heterostructures is the key aspect of this thesis. Therefore, direct current (DC) measurements were performed on different MASS diode structures.

A Source Measurement Unit (SMU 237) from Keithley serves as power supply. Furthermore, it is also equipped with sensitive Ampère- and Voltmeters in order to conduct precise current and voltage measurements. The investigated samples are mounted on a sample holder (section 4.2.2) and connected to the SMU. In this context, the bias-voltage or current can be applied to the samples in different probe geometries such as local and remote configuration which are described in detail in section 4.2.1.

Furthermore, the temperature of the samples can be regulated either by a lowtemperature setup inside a vacuum chamber at approximately $1 \cdot 10^{-6}$ mbar (see section 4.2.3) or by high-temperature setup inside a aluminum-box under ambient conditions (see section 4.2.4). In either case, the temperature can be monitored and regulated by a $\mathrm{PC}$.

Finally, the actual measurements can be conducted fully automated using the same PC. The procedure of data recording and processing as well as essential aspects of the controls are described in section 4.2.5.

\subsubsection{Probe geometry}

As mentioned above, there are two basic probe geometries applied to the samples. The local measurement (figure 4.3 a) is basically a two point setup. The bias of the SMU (output high) is connected to the top-contact of the sample, the ground (output low), on the other hand, to the back contact. The optional bias sensing ports of the SMU (sense) are short circuited. In this geometry, all resistances originating from contacts and wires outside the investigated heterostructure interfere with the original characteristic of the sample and are included in the recorded datasets. The remote configuration (figure $4.3 \mathrm{~b}$ ) requires two additional connections to the SMU and four separate contacts on the sample surface, two in each contact area, are mandatory. The power is supplied by two wires (output high and low) and decoupled from the voltage probing contacts (sense high and low).

For example, if there are high contact resistances present while recording a currentvoltage (I-V) characteristic the power supply will compensate by increasing the output bias until the desired voltage at the sense ports is reached. In this context, the voltage probing itself is not effected by high contact resistances up to values in the $\mathrm{M} \Omega$-range due to the internal resistance of the voltmeter. Furthermore, the current applied to the sample also increases at the same time. As a result, the recorded resistance data are significantly lower compared to the local setup and correspond to the actual heterostructure. Therefore, contact resistances due to poor contact quality as well as resistances of the wires can be neglected in a remote setup. However, 

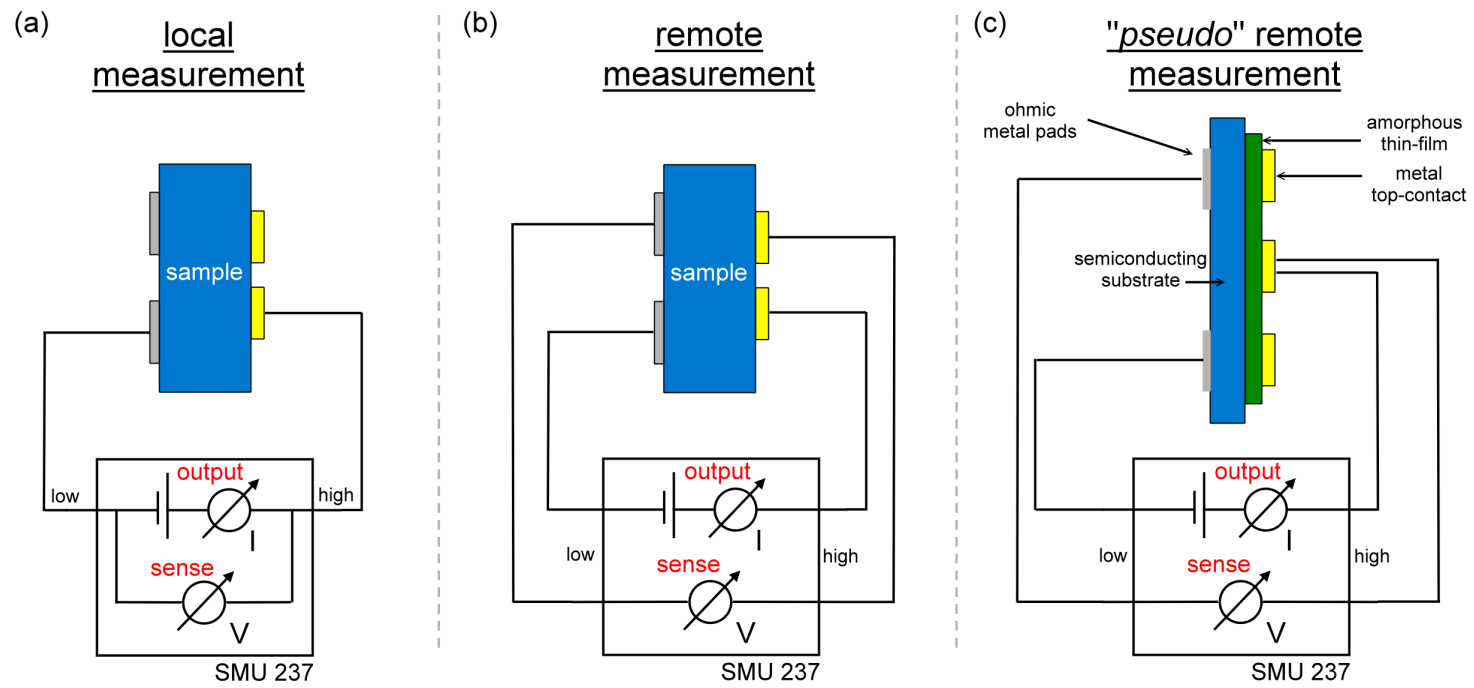

Figure 4.3: Probe geometries: (a) The local measurement - basically a two point setup. The bias of the SMU (output high) is connected to the top-contact of the sample, the ground (output low), on the other hand, to the back contact. The optional bias sensing ports of the SMU (sense) are short circuited. (b) The remote measurement - a four point setup. Compared to the local setup, two additional connections to the SMU are required and four separate contacts on the sample surface, two in each contact area, are mandatory. The power is supplied by two wires (output high and low) and adjusted on the basis of decoupled voltage probing contact measurements (sense high and low). (c) Pseudo remote measurement applied to a MASS heterostructure. The contact resistance of the back-contacts is eliminated. Output and sense high are short-circuited by the topcontact of the sample. In that way, the resistances of the cables are eliminated but the intended MASS heterostructure is preserved.

the geometry of the sample has to be considered in a four point probing configuration, in particular regarding the measurement of samples containing thin-films.

In this work, all investigated samples possess layered structures as described in chapter 2. Therefore, the substrate forms one contact area whereas the evaporated metal contact forms the opposite one. As this contact itself is part of the investigated heterostructure, an actual remote measurement with two separate top-contacts cannot be realized. As a result, so called pseudo remote measurements were performed (figure 4.3c).

In principle, the grounded wires of the SMU, output and sense low, are both connected to separate back contacts of the sample as in a normal remote measurement eliminating occurring contact resistances on the back side of the samples. However, the output as well as the sense high port are connected to the same top-contact of the sample. In that way, the resistances of the cables are eliminated but the intended MASS heterostructure is preserved.

As a closing remark, it has to be noted that the local configuration of the SMU is realized by just short circuiting the output and sense terminals inside the device in this thesis. 


\subsubsection{Mounting of samples}

The samples were mounted on a sapphire with evaporated chromium/gold ( $\mathrm{Cr} / \mathrm{Au})$ contact-pads on top (figure 4.4). The sapphire provides good thermal conduction properties but also insulation in terms of electrical conduction to grounded parts of the setup. The samples were fixed to the sample holder and connected to the contacts pads using liquid conductive silver preferably in the area of evaporated ohmic contacts (section 2.3).

The top contacts of the samples were connected to the contact pads on the sapphire using very thin aluminum wires, so called bonds. The Al-bonds were processed with a KULICKE \& SOFFA 4523 wedge bonder using Al wires with a diameter of $30 \mu \mathrm{m}$ at room temperature. In principle, a small wedge shaped hammer is vibrating at an ultrasonic frequency leading to a deformation of the Al-wire near the tip during contact. As a result it is bonded to the particular contact pad. For practical reasons,

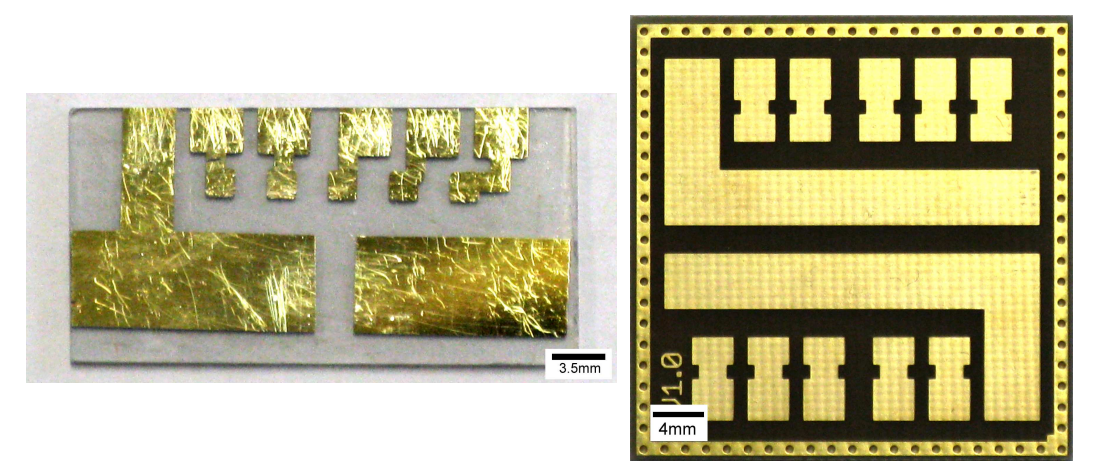

Figure 4.4: Sample Holders: sapphire with evaporated $\mathrm{Cr} / \mathrm{Au}$-contacts on top (left) and prefabricated sample-board with $\mathrm{Ni} / \mathrm{Au}$ contact-pads and an additional ground at the edge (right).

due to a limited number of contact pads, most of the top contacts are provided with just one $\mathrm{Al}$ bond (pseudo remote measurement configuration) leading to greater statistics of measured top contacts.

Experiments, conducted during this work, showed that the resistances of the wires and BNC connectors as well as the sample holder contacts are all below $5 \Omega$ and therefore negligible compared to the investigated samples. A typical setup is sketched for a true remote measurement in figure 4.5. In a few cases, such as due to delaminating contacts, $\mathrm{Au}$ pressure contacts were directly pressed onto the top-contact pads.

In addition to the sapphire sample holders, circuit boards with a similar contact shape and area, originally designed for impedance and photoresponse measurements, were used. The contacts pads are made up of nickel and gold. Furthermore, the boards are equipped with an additional ground at the edge, shown in the right image of figure 4.4 . 


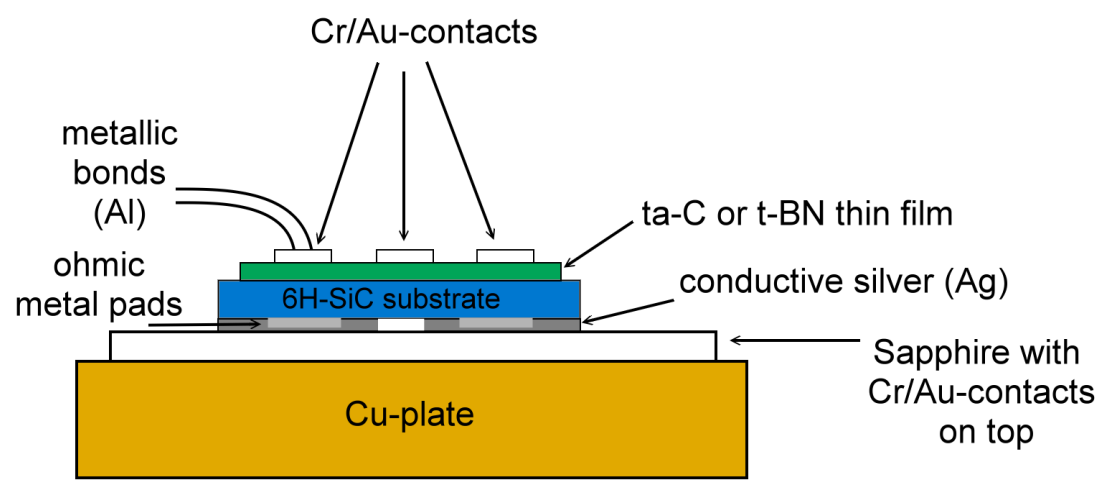

Figure 4.5: Electrical characterization setup (DC) of grown MASS heterostructures in remote configuration.

\subsubsection{Low-temperature setup}

In order to investigate and identify the conduction mechanisms occurring in heterostructures, it is essential to perform temperature dependent measurements, preferentially at low temperatures. Therefore, the sample holder is mounted inside a low-temperature setup as sketched in figure 4.6. In more detail, the sample holder is mounted on a $\mathrm{Cu}$-plate and fixed with conductive silver additionally providing an increase of thermal conductivity due to compensation of surface roughness at the interface.

The sample can be cooled down using a closed cycle helium cryostat from CTI Cryogenics. A spiral of indium (In) is pressed between the Cu-plate and the cold-head of the cryostat providing good thermal conduction. Furthermore, a $\mathrm{Cr} / \mathrm{Ni}$ heating wire $(R=50 \Omega)$ is wrapped around the $\mathrm{Cu}$-plat 11 providing an incremental compensation of thermal energy loss or even heating. The whole setup is mounted inside a vacuum chamber. The electrical contact between the SMU and the sample is realized using a total of six electrical vacuum feedthroughs. The vacuum conditions are provided by a turbo-molecular pumping unit at pressures of $\left(5 \cdot 10^{-7}-3 \cdot 10^{-6}\right)$ mbar. In addition, the thermal radiation of the surrounding chamber is reduced by an additional cold shielding. In this setup, $\mathrm{I}-\mathrm{V}$ measurements in the temperature range of (14-300) K can be realized.

During the experiment, the temperature is monitored and adjusted by a PID control unit, a LAKESHORE 331 Temperature Controller. The temperature is measured by the temperature controller using two DT-470-CU-12 semiconductor temperature diodes from Lake Shore which are fixed to the $\mathrm{Cu}$-plate as well as to the cold head. In order to reach the desired temperature, the heating wire is supplied by the temperature controller with a suitable percentage of the maximum power output. The whole process can be controlled remotely by a PC via an IEEE-interface. That way the measured temperature data can also be transferred.

\footnotetext{
${ }^{1}$ and fixed with thermally high conducting but electrically insulating compound, called Stycast
} 


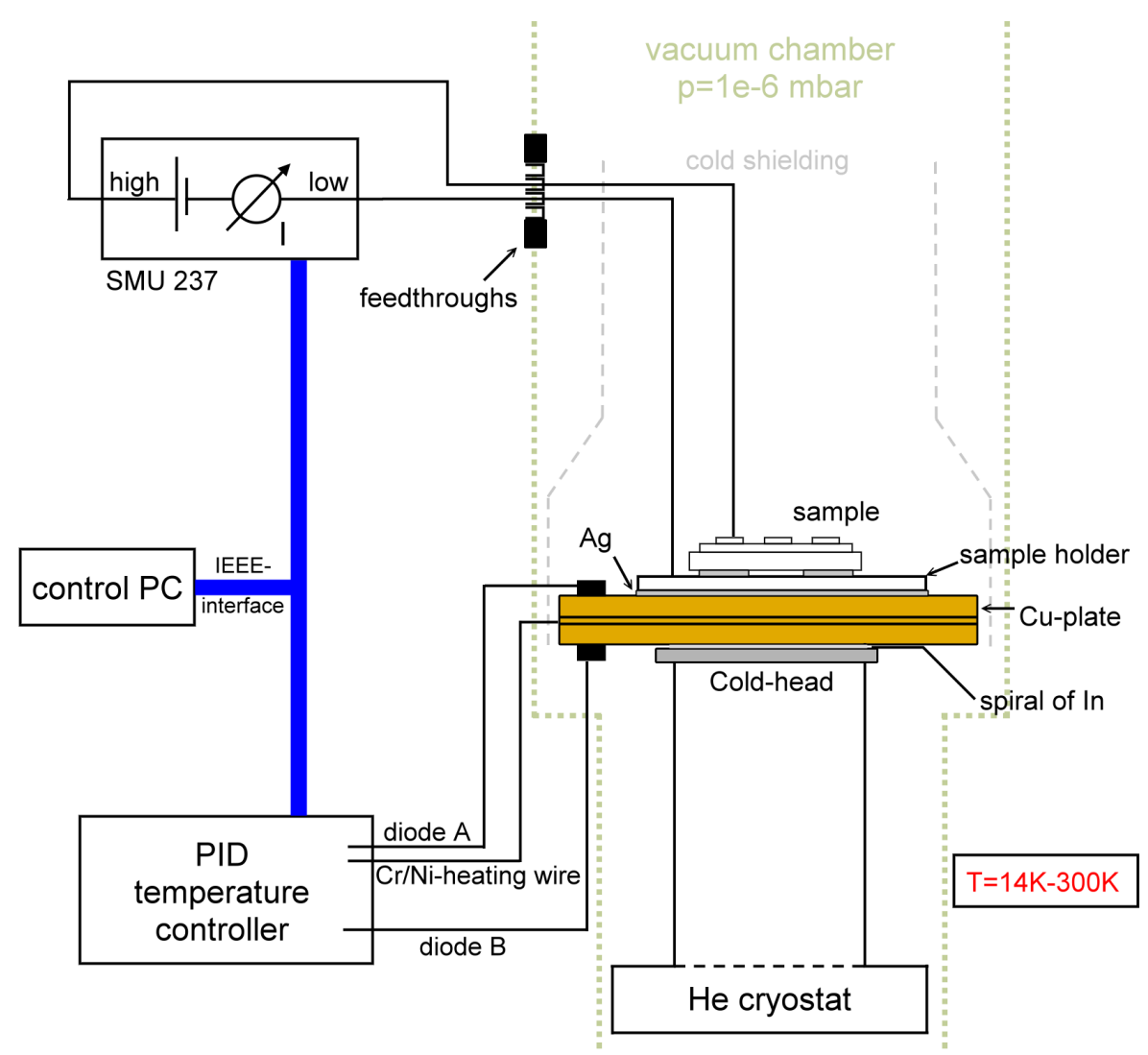

Figure 4.6: Low-temperature setup. The whole setup is mounted inside a vacuum chamber. The sample holder is mounted on a $\mathrm{Cu}$-plate and fixed with conductive silver additionally providing an increase of thermal conductivity due to compensation of surface roughness at the interface. The sample can be cooled down using a closed cycle helium cryostat. A spiral of indium (In) is pressed between the $\mathrm{Cu}$-plate and the cold-head of the cryostat providing good thermal conduction. Furthermore, a $\mathrm{Cr} / \mathrm{Ni}$ heating wire is wrapped around the $\mathrm{Cu}$-plate providing an incremental compensation of thermal energy loss or even heating. The temperature is monitored and adjusted by a temperature controller with an integrated PID control unit. In addition, the electrical connection between the SMU and the sample are realized using a total of six electrical vacuum feedthroughs. The whole process can be remotely controlled by a PC via an IEEE-interface. 


\subsubsection{High-temperature setup}

For high-temperature measurements in a temperature range of (300-650) K the cryostat is replaced by an appropriate setup shown in figure 4.7. The sample holder is mounted inside a small aluminum box (gray dashed line) to reduce the temperature gradient between the sample and the ambient air. Furthermore, it is connected to the SMU using Au pressure contacts. The heat is provided by a hot plate. The temperature is monitored by a Voltcraft K204 data-logger using thermocouples (type-K). The whole setup is mounted inside a "black-box"'2 under ambient condi-

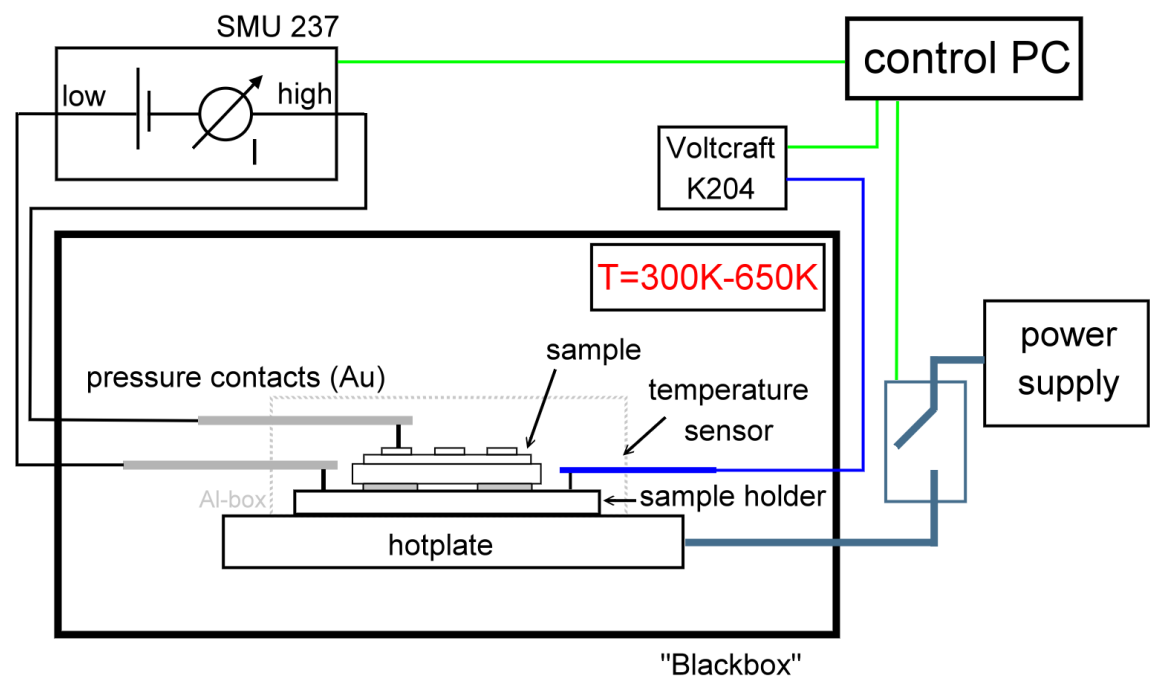

Figure 4.7: High-temperature setup. The sample holder is mounted inside a small aluminum box (gray dashed line) and connected to the SMU using Au pressure contacts. The heat for measurements in the temperature range of $300-650 \mathrm{~K}$ is provided by a hot plate. The temperature is monitored by a Voltcraft K204 data-logger using thermocouples (type-K). In addition, the power supply can remotely be turned off using a PC-controlled switch.

tions. Prior to temperature dependent measurements, this black-box setup was also used for room-temperature measurements, testing of the bond contact quality as well as verification of stable contact quality (top and back) of the samples.

During the experiments, the heating process, temperature monitoring and data recording are controlled by a $\mathrm{PC}$. The temperature is usually increased at a rate of (1-2) K/min, depending on the setting of the power supply. An I-V measurement is remotely started and recorded right after the desired temperature is reached and takes less than $10 \mathrm{~s}$. Therefore, the temperature can be assumed to be constant during the measurement $(\Delta T \approx 0.1 \mathrm{~K})$. After reaching the maximum temperature of the measurement, the power supply is remotely cut and the temperature of the sample decreases at a much slower cooling rate compared to the heating curve. During the cooling process additional measurements are performed and recorded at the

\footnotetext{
${ }^{2}$ another Al-box, further shielding the setup from emerging temperature gradients as well as from irradiation of light
} 
same temperatures in order to verify the previous measurements.

In principle, HT measurements complement room and low temperature measurements leading to a comprehensive study of the underlying conduction mechanisms. In particular, HT measurements are useful regarding conduction mechanisms dominated by thermionic emission.

\subsubsection{Data recording and processing}

All of the measurement setups mentioned above are controlled, monitored and read out by a PC. They can be performed manually as well as in automated process cycles using a user interface (UI). The control commands as well as data obtained from the SMU and the Lake Shore temperature controller are transferred via an IEEE-interface. The Voltcraft K204, on the other hand, is connected and read out via a COM-port.

All control commands for the different devices used in the process are provided by python scripts, written in Python 2.7 [133], and the user interface is coded in WXPython 2.8 [134]. All applied python scripts in this thesis were written by H.-G. Gehrke and subsequently edited by the author in some cases.

Regarding the limited number of vacuum feedthroughs as well as contact pads on the sample holders usually only a "pseudo" remote measurement is performed in most of the measurements. In this context, the setup is equipped with a so called

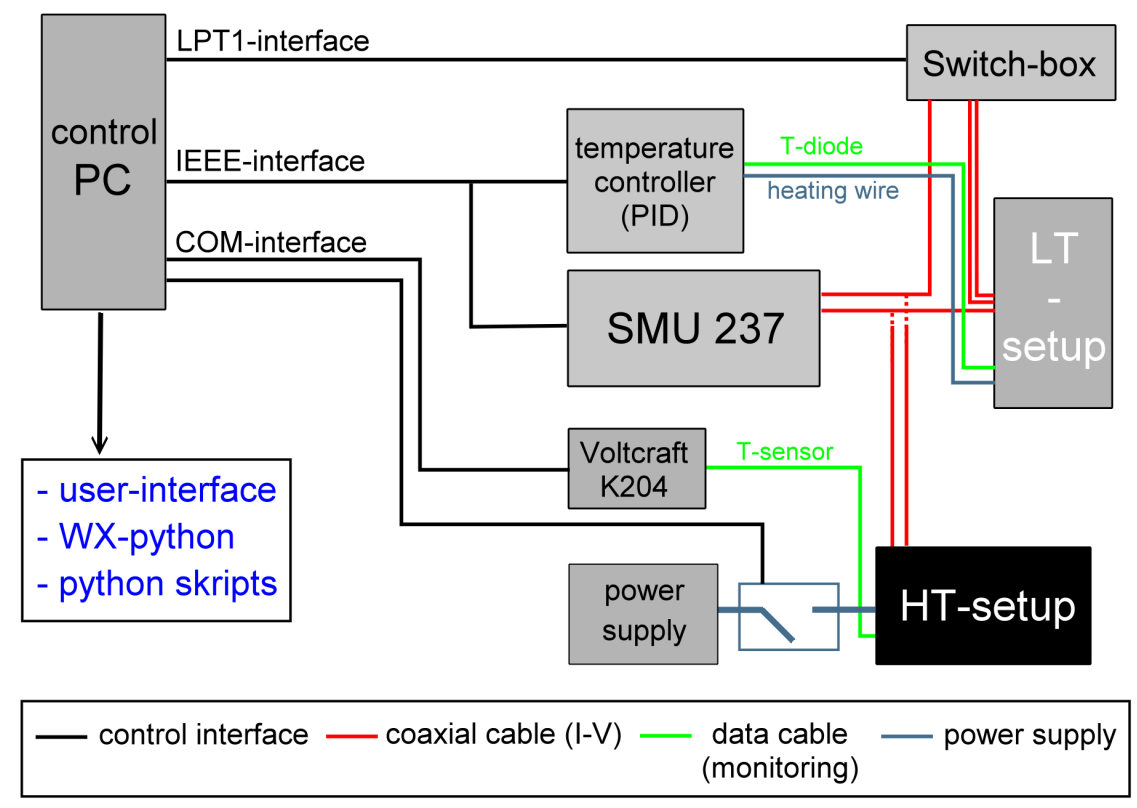

Figure 4.8: Basic layout of the data-processing setup.

switch-box, a remotely controlled relay-array, ensuring the investigation of different top contacts of one sample at identical conditions. Each real top-port is assigned to a so called parport of the switch-box. The switch-box itself is controlled via the 
LPT1 port of the PC and connected to the set of output and sense high.

During measurements, the compliance of the SMU is usually set to the maximum value of $100 \mathrm{~mA}$. If samples are effected by high currents, the compliance can be decreased using the UI. Furthermore, an additional delay between measurement steps can be applied in the ms and s range to avoid or suppress charging effects during measurements. The UI also permits free control of parameters like step-size, limits, integration time, number of averaged datasets and free choice of local or remote measurement. Regarding temperature dependent measurements, the exact temperature steps as well as the sequential order of heating and cooling can also be controlled using the UI (LT) or another python script (HT). In order to verify the reproducibility of the performed measurements at least 3 characteristics were recorded for each temperature step in the low temperature measurement. Due to the duration time of approximately $10 \mathrm{~s}$ and continuous heating at the same time, only one measurement is taken at each temperature step in the HT measurement. After completing a full temperature cycle, corresponding datasets of a certain temperature are assigned to each other and averaged using another python script 3 . In these final output files, the I-V data are sorted by temperature in separate files for each measured parport.

In addition, these datasets can also be transposed in such a way that the current can be plotted as a function of temperature at a fixed bias-voltage. The whole process of data-recording is sketched in figure 4.8 .

\footnotetext{
${ }^{3}$ auswertung 1.08 written by H.-G. Gehrke
} 


\subsection{Impedance spectroscopy AC}

In addition to the direct current measurements, described above, the AC-properties of all different MASS heterostructures have been investigated by measuring the complex impedance as a function of bias voltage, comparable to the DC mode, as well as of frequency in the range of $20 \mathrm{~Hz}$ to $1 \mathrm{MHz}$.

The measurements were performed using a Hewlett-Packard HP4284A auto-balance bridge (figure 4.9 (left)). It was operated in a so called Z-theta (deg) mode measuring the complex series impedance $\underline{Z}=|\underline{Z}| \exp (i \theta)$ and recording values of the impedance $|\underline{Z}|$ and the phase $\theta$.

Therefore, an impedance spectroscopy measurement at alternating currents and different frequencies provides insight into the frequency dependence of impedances and corresponding phase information regarding the characteristics of MASS heterostructures as well as information about emerging capacitances in comparison to direct current measurements. The measurements were performed using an automated control program with a command line interface (CLI) providing an input of file name, operator, bias range and bias step sizet. The properties of this setup as well as the softwart5 are described in detail in the work of J. A. Amani [135].

Regarding the actual measurements of this work, all of them have been performed without the use of any correction functions provided by the HP4284A due to unreasonable results in case study experiments when using them. In order to verify the $\mathrm{AC}$ characteristics, obtained under these conditions, additional measurements were performed on almost pure capacitive and pure resistive reference samples. Both of them led to reasonable results.

During the measurement, the sample holder (see section 4.2.2) is mounted in an additional fixture (see figure 4.9 (right)) surrounded by a boxed Al-housing (left) screening the setup in order to ensure minimal interference from external electromagnetic sources. In addition, stray capacitances, inductances and resistances were minimized. The fixture consists of a layered structure of grounded, insulating and conducting parts. The top layer is made up of another board equipped with pressure contacts made up of bronze in order to connect the pads on the sample holder. The power is supplied by the auto-balance bridge through a serial arrangement of BNC-jack 6, coaxial cables and MMCX connectors on the connector board.

The working principle of the auto-balance bridge and corresponding circuit diagrams as well as detailed specifications of the sample fixture and screening are also provided in reference [135]. In principle, the mounting of the sample is very similar compared to a DC measurement in a true remote configuration. Therefore, some top-contacts were provided with an additional Al-bond. However, the back-contacts of the samples are additionally short-circuited using a small amount of conductive silver in order to avoid additional resistances and capacitances, eventually caused by

\footnotetext{
${ }^{4}$ all saved in an additional log file

${ }^{5}$ coded in python 2.7 by J.A. Amani

${ }^{6}$ all crimped inside the boxed housing
} 

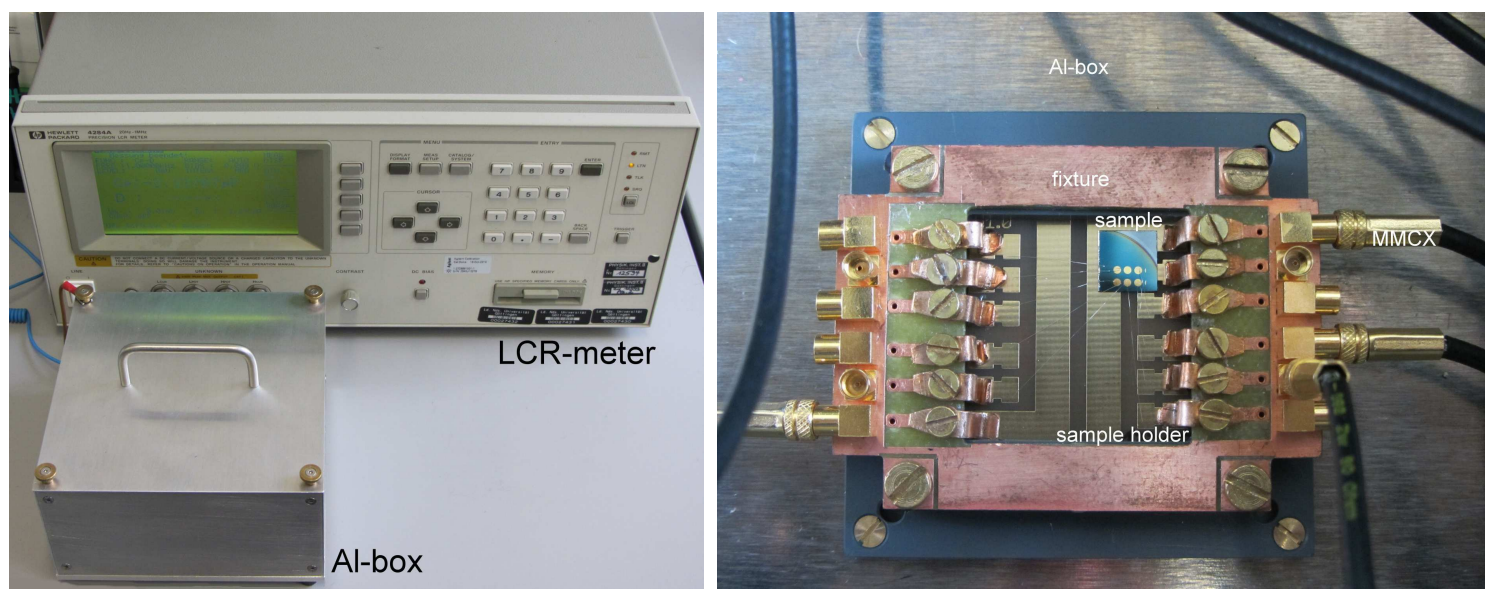

Figure 4.9: Impedance spectroscopy setup: (left) LCR-meter - a Hewlett-Packard HP4284A auto-balance bridge. The sample is surrounded by a boxed Al-housing in order to screen the setup and ensure minimal interference from external electromagnetic sources. (right) The top-contacts of the sample are equipped with two Al-bonds each, the back-contacts of the samples are shortcircuited using a small amount of conductive silver. The sample holder is mounted in an additional fixture and connected to the auto-balance bridge via MMCX-jacks.

a low conducting substrate or two Schottky contacts on the back side of the sample which would interfere with the characteristic of the investigated structure even more and might lead to an unbalanced system during the measurement. As a result of the short-circuit, the characteristic is basically comparable to a local measurement configuration of the same sample in DC mode.

The actual AC-measurement was typically performed right after the temperature dependent DC measurement. The limits in bias-voltage were determined in the foregoing DC I-V measurements. The integration time is set to "long" and the oscillator level to $100 \mathrm{mV}$.

\footnotetext{
${ }^{7} 100 \mathrm{mV}$ on the display is equivalent to an rms amplitude of $141 \mathrm{mV}$
} 


\subsection{Photoresponse measurements}

As mentioned in section 3.8, several semiconductor-based structures show a photoresponse when exposed to light. In the following, different illumination procedures as well as the corresponding setup and recording techniques are described in detail.

\subsubsection{Case study experiments}

In a case study work step, samples were biased in reverse and, in some cases, also in forward direction and exposed to the light of a "white" LED. It was placed at a distance of about $1 \mathrm{~mm}$ and an irradiation angle of approximately $25^{\circ}$ (see figure 4.10). Regarding measurements of different heterostructures the same LED

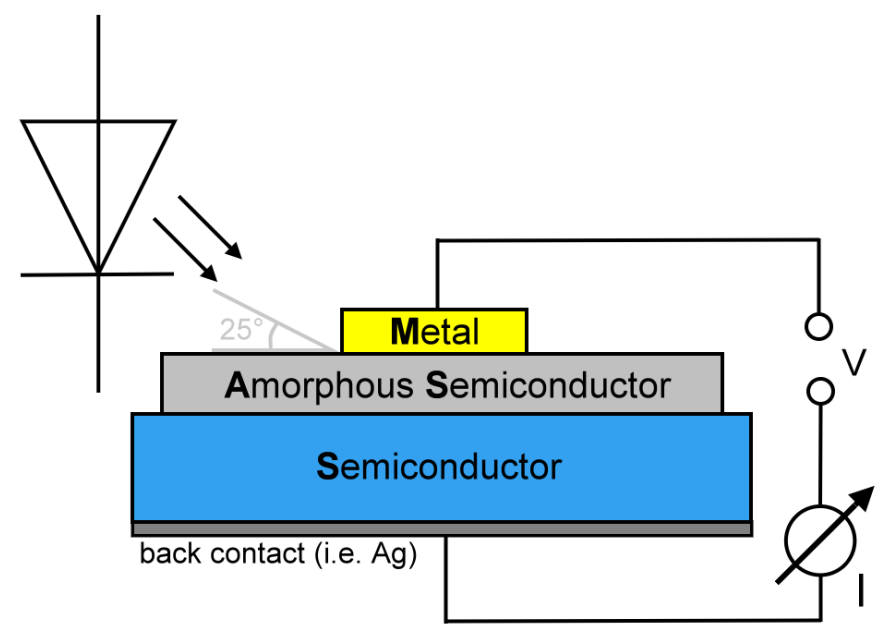

Figure 4.10: Photoconductivity setup of case study measurements. The samples were biased in reverse and, in some cases, also in forward direction and exposed to the light of a "white" LED. The resulting photo-response was investigated by recording the current of the samples as a function of time using a SMU.

was always operated at a constant power $(3.12 \mathrm{~V}, 0.011 \mathrm{~A})$ in order to ensure reproducibility of the measurements. As shown in a cathodo-luminescence spectrum in figure 4.11, the LED exhibits a sharp blue line at a wavelength of around $450 \mathrm{~nm}$ and a rather broadened green line at $550 \mathrm{~nm}$.

The samples were exposed for $10 \mathrm{~s}$ and $100 \mathrm{~s}$ at different bias-voltages. The resulting photo-response was investigated by recording the current of the samples as a function of time using a SMU (see section 4.2). The minimal time-resolution of the SMU 237 is approximately $20 \mathrm{~ms}$. In each measurement, the data recording was continued until equilibrium was reached. After that, the sample was exposed again for a different duration of time. 


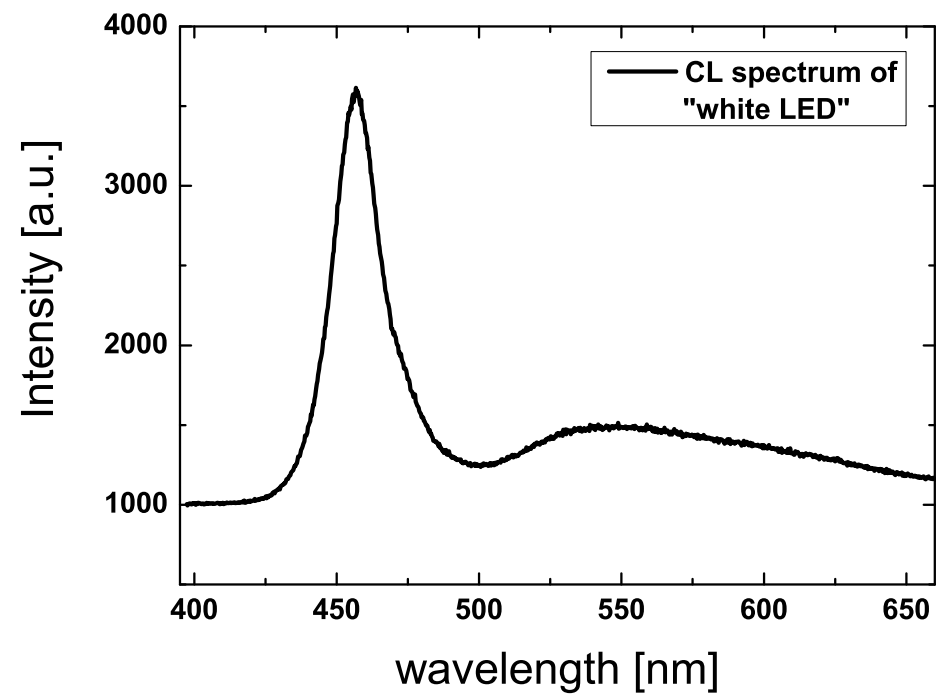

Figure 4.11: Cathodo-luminescence spectrum of a white LED used for case study photo-response measurements. The LED exhibits a sharp blue line at a wavelength of around $450 \mathrm{~nm}$ and a rather broadened green line at $550 \mathrm{~nm}$. 


\subsubsection{Time-resolved photoresponse}

Due to the fact that the time resolution of the SMU is limited to the high ms range, a new setup was designed in the work of Amani et al. [135] using a pulsed Nd:YAG laser in the nanosecond range, another SMU as power supply and an oscilloscope in order to investigate photoresponses with a time resolution of some nanoseconds.

In principle, a MASS heterostructure, biased by the SMU, is irradiated by discrete pulses of a laser-beam at two different wavelengths (green and UV). The resulting photoresponse is monitored and recorded using an oscilloscope with time-resolution in the nanosecond range while being triggered by the laser-setup. Furthermore, the sample holder fixture of the setup is identical to that of the impedance spectroscopy setup but is equipped with two additional holes to mount it on a XZ-stage. In order

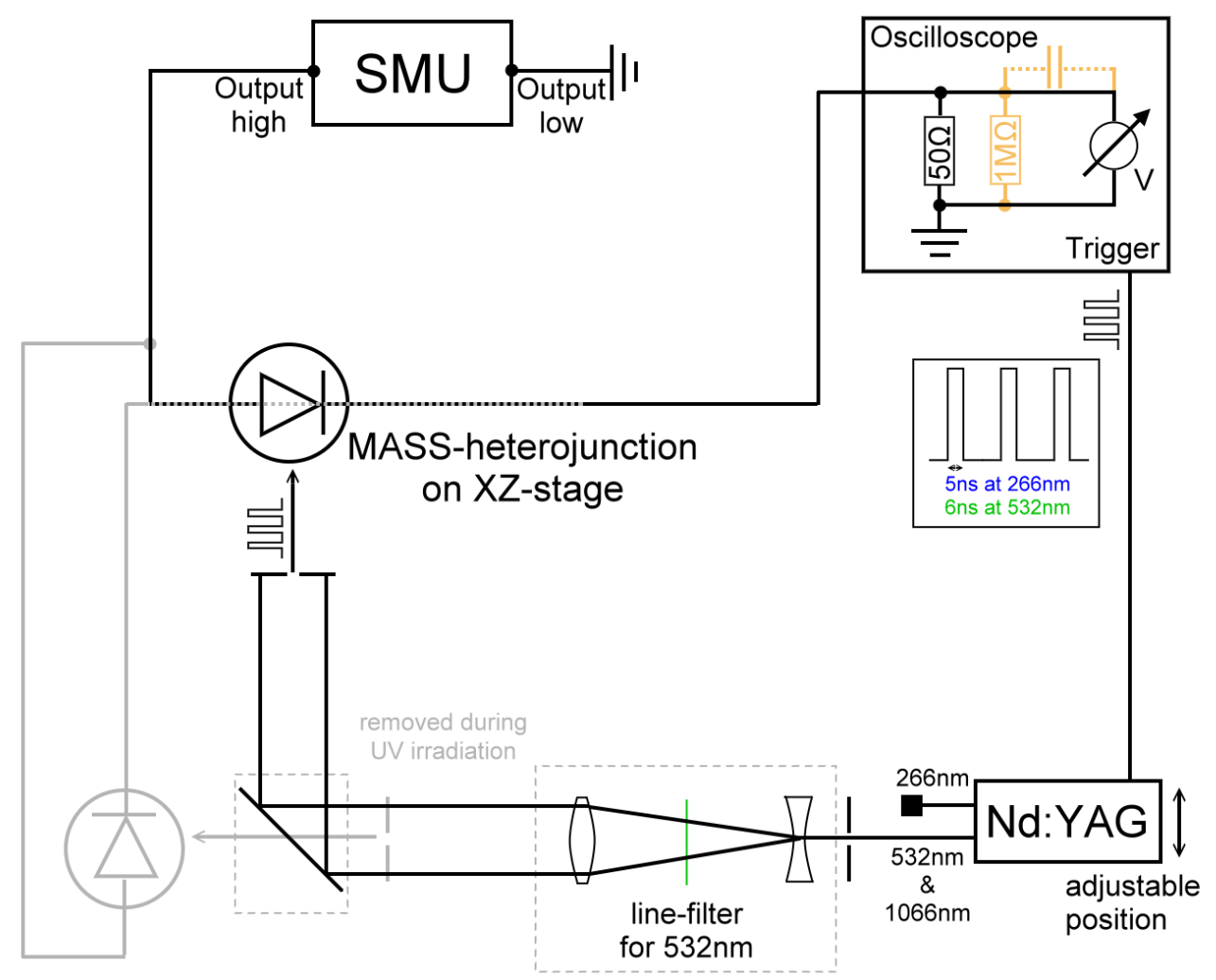

Figure 4.12: Time-resolved photoresponse setup. The samples are illuminated using a pulsed Nd:YAG laser providing wavelengths of $266 \mathrm{~nm}, 532 \mathrm{~nm}$ and $1064 \mathrm{~nm}$. Focusing on the setup for the green line at $532 \mathrm{~nm}$, the accompanying infrared line is screened from the laser beam using a laser line filter (green dash). Due to alignment and intensity reasons, the laser beam is widened and parallelized by lenses in the optical pathway and further lowered in intensity by two iris diaphragms. The sample is biased by a SMU and the position can be adjusted using a XZ-stage. The input impedance of the oscilloscope is set to $50 \Omega$. The ground potential of the oscilloscope is connected to the SMU low output by shortening the inner and outer conductor of the coaxial cables. The trigger of the oscilloscope was connected to the Q-switch sync trigger of the laser. The UV-setup as well as deviations from the original setup are marked gray. An optional input impedance (Osc.) of $1 \mathrm{M} \Omega$ and the corresponding coupling capacitance are marked orange. 
to hit the sample at the desired spots (top contacts), the XZ-position of the sample holder was adjusted and the optical path of the setup was fixed. The whole setup is sketched in figure 4.12 .

Regarding the essential components of the setup in more detail, the laser, a Quantel Ultra 75, operates at an original wavelength of $266 \mathrm{~nm}$ leaving the casing through a designated aperture. After reflection by 2 dichroic mirrors the wavelength is also doubled and quadrupled, respectively, leading to two new laser lines at wavelengths of $532 \mathrm{~nm}$ (green) and $1064 \mathrm{~nm}$ (infrared). Both of them leave the laser casing through an additional aperture right next to the UV exit. The green laser-light can be separated from the infrared by using a laser line filter from Thorlabs, a FL532-10, with a central wavelength of $(532 \pm 2) \mathrm{nm}$ and a full width half mean of $(10 \pm 2) \mathrm{nm}$. As the power of the laser exceeds the damage threshold of the filter even at its lowest setting, the intensity of the beam has to be lowered. Therefore, the beam was widened using a biconcave lens with a focal length of $f=-20 \mathrm{~mm}$. After passing the filter, the divergent beam was parallelized using an achromatic lens with a focal length of $f=250 \mathrm{~mm}$. Two iris diaphragms were additionally used to align the optical pathway and, on the other hand, to further reduce the beam-intensity of the laser. Furthermore, a tilted mirror was used to reflect the beam by $90^{\circ}$ right onto the sample. Finally, an additional iris diaphragm reduces the diameter of the beam to about (1.5-2) $\mathrm{mm}$ so that the particular top-contacts are fully illuminated during the measurement. In this context, a TV-camera is used to adjust the XZ-stage and match the laser beam to the top-contacts of the samples.

In addition, the samples are also illuminated using the UV-line at $266 \mathrm{~nm}$. Due to absorption of the UV-light inside the lenses and possibly resulting damage they are removed from the optical pathway (dashed gray lines in figure 4.12). The laser pulses are only aligned by using the iris diaphragms and, in the process, reduced in intensity. The tilted mirror is removed and the XZ-stage placed right into the optical pathway of the laser-beam (also marked gray).

The position of the sample is adjusted by using an UV-sensitive sheet of paper. In addition, due to the lack of an infrared laser-line filter this wavelength could not be measured during this thesis.

During the measurements, the samples are biased at various voltages using a Keithley 2611A SMU. Even at zero bias the SMU is in operating mode for interference and correction reasons. Furthermore, all samples are connected in a local setup configuration and mounted in the fixture described above. As explained in section 4.3, the fixture is connected to the SMU via MMCX jacks and the sample holder itself is connected using pressure contacts made up of bronze.

One port of the sample is connected to an input port of an oscilloscope, a Tektronix DPO4104, and eventually to the ground potential. The other port is connected to the output high of the SMU. By shortening the inner and outer conductor of the coaxial cables the ground potential of the oscilloscope is connected to the SMU low output. That way the ground potential of the SMU and the oscilloscope are linked to each other. As a result, a change in polarity of the SMU (negative bias-voltage) 
causes a change in ground potential. Therefore, the sample is always connected in such a way that a positive bias-voltage is applied. In order to choose between forward and reverse bias direction of a sample, the ports have to be switched right at the connectors of the fixture.

The input impedance of the oscilloscope which is also the resistor of the photoresponse measurement can be set to $50 \Omega$ and $1 \mathrm{M} \Omega$ (marked orange). In addition, it is equipped with an optional coupling capacitance.

The trigger of the oscilloscope was connected to the Q-switch sync trigger of the laser. During the experiments, several repetition rates of 1, 2, 5, 10 and $20 \mathrm{~Hz}$ are available and are chosen depending on the maximal decay time of the particular sample. Furthermore, the Nd:YAG laser exhibits pulse times of 6 ns at $532 \mathrm{~nm}$ wavelength and $5 \mathrm{~ns}$ at $266 \mathrm{~nm}$. As the trigger signal of the laser possesses two rising edges at a very low time-interval instead of one, a trigger hold-off was set to $500 \mu \mathrm{s}$.

On every laser pulse, the voltage drop at the input impedance was recorded as a function of time. In addition, the rise and decay times of each recorded dataset are determined by the software of the oscilloscope. Per definition, this is the time until the signal changes between $10 \%$ and $90 \%$ of the maximum amplitude of the signal. In this thesis, only the mean value of 512 single measurements is taken to counter the effect of time jitter caused by the laser. However, the actual time jitter regarding the datasets of the whole response curve cannot be eliminated. Nevertheless, the signal averaging routine of the Tektronix DPO4104 is also used here and an average response characteristic is recorded as a mean value of 512 sweeps. As a result, due to the time jitter, the averaged response signal is significantly broadened compared to a single measurement. For comparison, some of the single measurements are also recorded.

In addition, the electromagnetic pulse caused an induction into the leads of the setup leading to additional oscillations and interference with the original response signal. Therefore, a reference measurement was taken and averaged for a period of 512 pulses while the laser-beam was shuttered from the optical pathway. Then, the obtained data was subtracted from the signal of the actual measurement. 


\section{Chapter 5}

\section{Structural characterization}

\subsection{Introduction}

The structural properties of the heterostructures were investigated in two steps. Right after deposition, the thin-films were investigated using XPS-measurements (see section 4.1.1) in order to verify the intended type of phase (t-BN) or fraction of phases (ta-C).

After the completion of all electrical measurements, at least one sample of each type of heterostructure was investigated using transmission electron microscopy (TEM, see section 4.1.2) in order to study their crystalline micro-structure as well as to determine the correct film thicknesses compared to the calculated values (see equation 2.1 in section 2.5.3).

\section{$5.2 \quad$ XPS-measurements}

X-ray photoemission spectroscopy (XPS) measurements were performed in order to determine the chemical composition of the grown thin-films as well as the dominating crystallographic phase.

Preliminary to the actual measurement, the detector was calibrated by measuring an Au-reference target. In order to avoid possible contaminants on the surface, the gold target was sputter-cleaned prior to the measurement using the typical charge of $15 \mathrm{mC} \mathrm{Ar}{ }^{+}$-ions at an energy of $1 \mathrm{keV}$. By calibrating the setup, a possible energy shift of a single channeltron can be compensated by correcting the data of the actual XPS-measurements. For this purpose, the ${ }^{4} f_{5 / 2}$ and ${ }^{4} f_{7 / 2}$ orbitals of $\mathrm{Au}$ with lines at binding energies of $84.0 \mathrm{eV}$ and $87.7 \mathrm{eV}$ were chosen [136]. A typical spectrum of a corresponding channeltron is shown in figure 5.1. 
The peak-positions were determined using a Gaussian distribution fit (see figure 5.1b). The binding energies were subsequently subtracted by the values obtained from the detector software. After that, a mean value of the displacement regarding both Au-peaks was calculated for each channeltron. The resulting offset-values were subsequently used to correct the actual XPS datasets of each channeltron. Therefore, the datasets were finally averaged in order to improve the signal to noise ratio of the measurements particularly at low statistics.
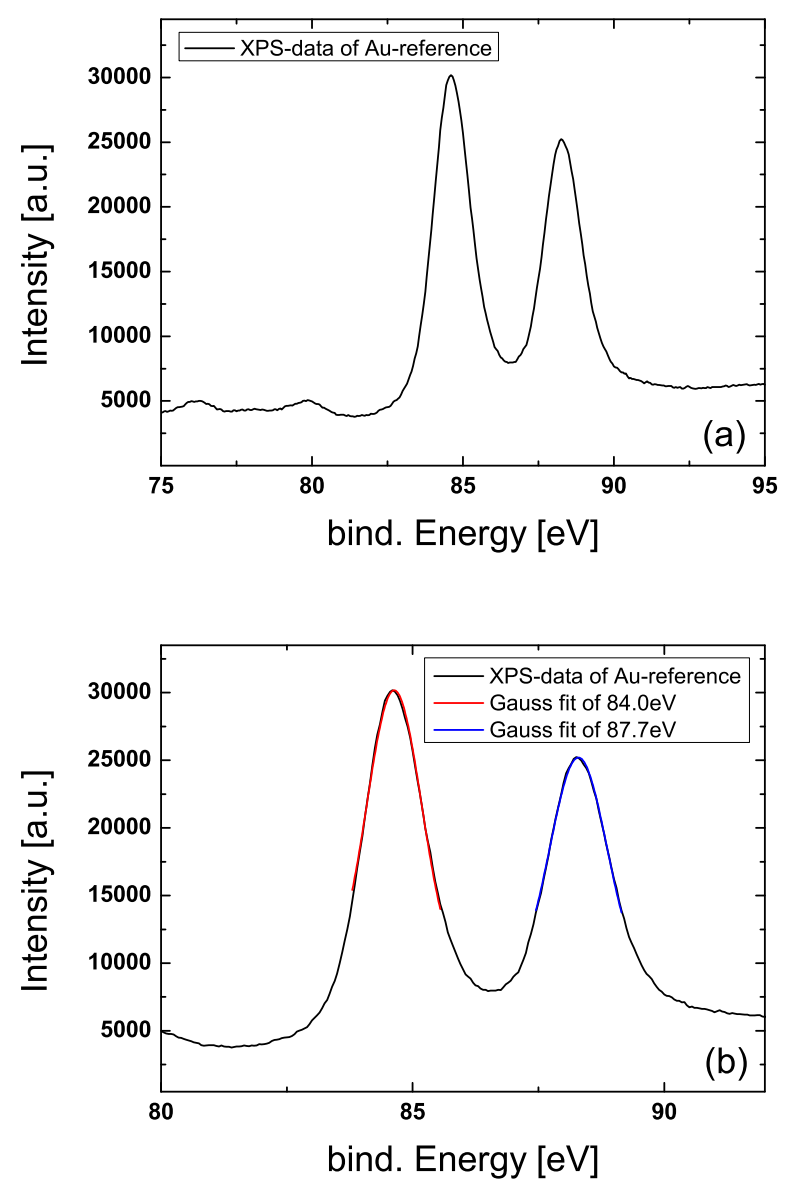

Figure 5.1: (a) Typical XPS-Spectrum of an Au-reference sample. (b) The peak-positions of the $\mathrm{Au}{ }^{4} \mathrm{f}_{5 / 2}$ and ${ }^{4} \mathrm{f}_{7 / 2}$ orbitals with lines at binding energies of $84.0 \mathrm{eV}$ (red) and $87.7 \mathrm{eV}$ (blue) were determined using a Gaussian distribution fits. 


\subsection{1 ta-C samples}

As mentioned in section 2.4.1, ta-C thin-films were deposited at $100 \mathrm{eV}$ ion energy onto the semiconducting substrates leading to a $\mathrm{sp}^{3}$-ratio of about $80 \%$.

In order to verify this radio between $\mathrm{sp}^{2}$ - and $\mathrm{sp}^{3}$-bonds in the first few nanometers of the amorphous carbon matrix, XPS-measurements were performed at an energy range around the $\mathrm{C}-1 \mathrm{~s}$ peak. Depending on the type of bonding, this peak is located at a binding energy of $284.4 \mathrm{eV}$ for graphite and at $285.2 \mathrm{eV}$ for diamond according to Merel et al. [137]. For an amorphous diamond-like carbon film such as ta-C, the recorded XPS-spectrum of the carbon peak has an intermidiate binding energy as it contains both types of bonding. Therefore, it can be fitted by a sum of two Gaussian distributions leading to an estimate of the $\mathrm{sp}^{3}$ - to $\mathrm{sp}^{2}$-ratio of the films. In this context, it has to be noted that the detectors have been near the end of their lifetime circle resulting in a limited energy resolution while suffering an increased noise-ratio of the spectra at the same time. Therefore, a more accurate method to determine the type of phase inside of the grown thin-film using XPS is the calculation of the plasmon energy. Regarding equation 4.2, plasmon excitations reduce the kinetic energy of the electrons and can therefore be found at higher binding energies with a corresponding energy difference.

According to Berger et al. [138], the plasmon energy of a ta-C thin-film has a typical value of around $30 \mathrm{eV}$ and is in-between the plasmon energies of purely $\mathrm{sp}^{2}$ and $\mathrm{sp}^{3}$ bonded carbon with an energy difference of $26.0 \mathrm{eV}$ and $33.8 \mathrm{eV}$, respectively.

In this context, the plasmon energy is directly proportional to the plasmon-frequency $\omega_{\mathrm{P}}$ according to equation 4.2. Based on a harmonic oscillator approximation regarding the motion of the electron gas, the plasmon frequency $\omega_{\mathrm{P}}$ is directly proportional to the square root of the electron density: $\omega_{\mathrm{P}} \propto \sqrt{n_{e l}}$ [64]. As a result, the plasmon energy can be directly linked to the electron density and therefore to the corresponding hybridization states of bonds inside the material.

Typical XPS-spectra of a ta-C film grown in this work are shown in figure 5.2 . The position of the $\mathrm{C}$ - 1 s peak has been determines to a value of $285.0 \pm 0.1 \mathrm{eV}$ using only a single Gaussian distribution fit (figure 5.2 $\mathrm{a}$ ). The low energy difference compared to pure diamond and the fact that the $\mathrm{sp}^{2}$-ratio could not be determined, eventually as a result of insufficient detector resolution, indicate a high $\mathrm{sp}^{3}$-ratio. Furthermore, the position of the plasmon peak (figure $5.2 \mathrm{c}$ ) and the resulting energy difference was sufficiently determined by using another Gaussian distribution leading to a value of $31.58 \pm 0.05 \mathrm{eV}$. These values are in good agreement with literature. Therefore, the desired high $\mathrm{sp}^{3}$-content of the grown ta-C thin-films has been verified. In addition, all relevant energy values are consistent for the entire stock of grown ta-C samples at random measurements. 

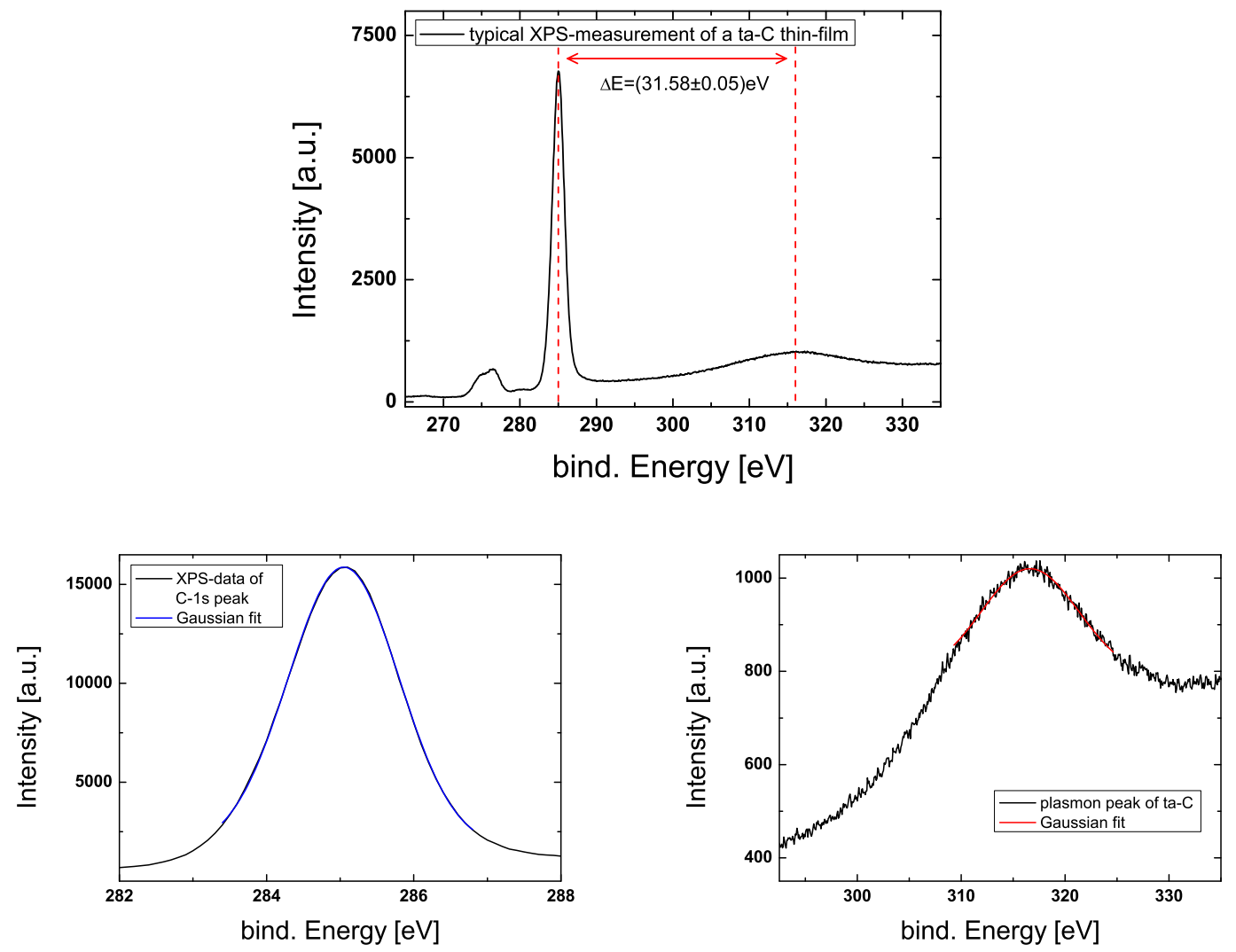

Figure 5.2: Typical XPS-spectrum of a typical ta-C thin-film. The survey spectrum around the $\mathrm{C}-1$ s peak of carbon is shown in (a). In addition, the position of the $\mathrm{C}-1 \mathrm{~s}$ (b, blue) as well as the corresponding plasmon peak (c, red) has been determined using Gaussian distributions. 


\subsection{2 t-BN samples}
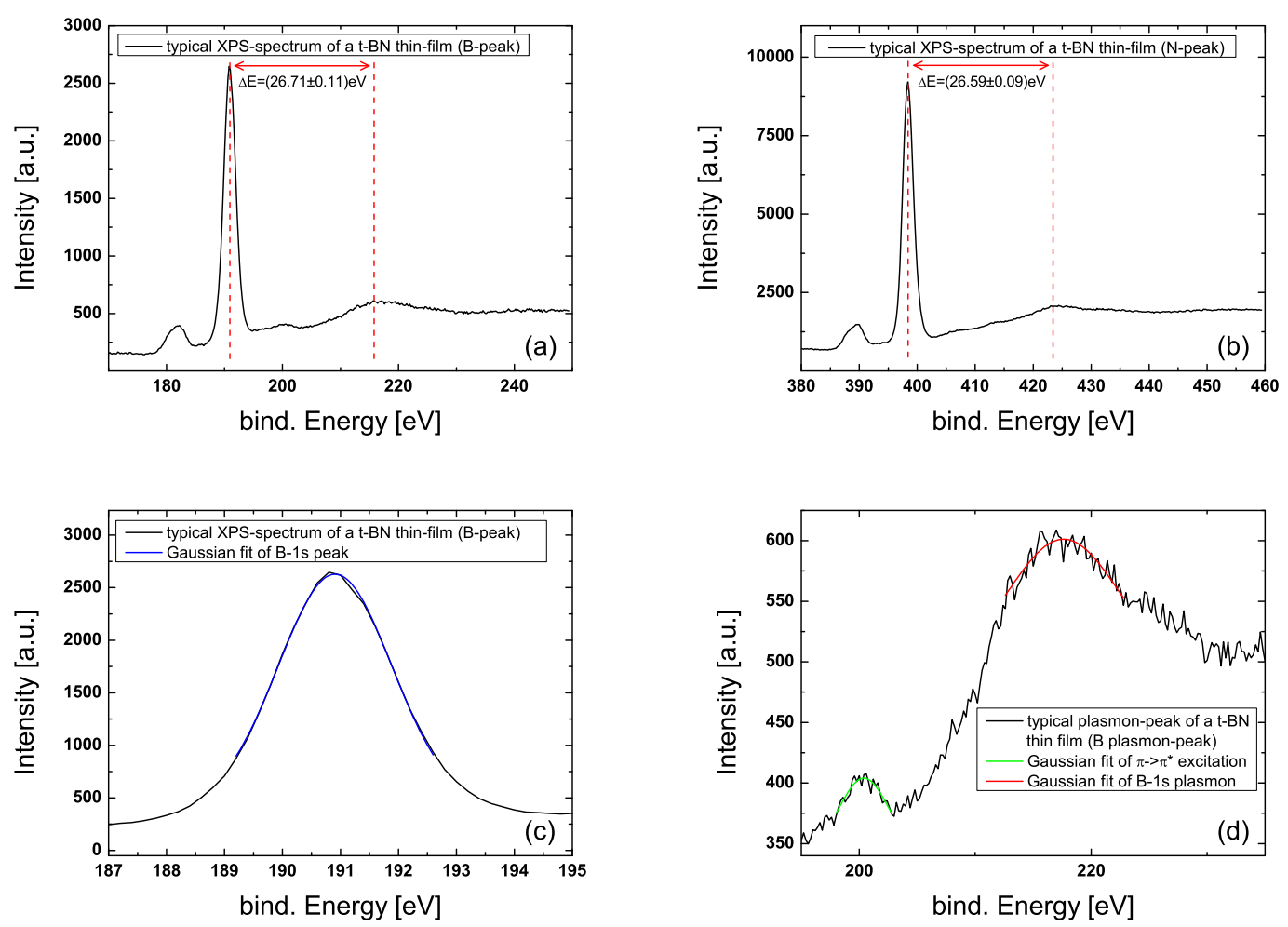

Figure 5.3: Typical XPS-spectrum of a typical t-BN thin-film. In addition to survey spectra of $\mathrm{B}(\mathrm{a})$ and $\mathrm{N}(\mathrm{b})$, the analysis method using Gaussian distribution is illustrated on the basis of B (c and $\mathrm{d}$ ). The peak of the inter-band excitation is marked green while the plasmon peak is marked red.

The XPS-measurements of the t-BN thin-films have been performed according to the procedure described in the section above. In comparison to ta- $\mathrm{C}$, the turbostratic boron nitride is exclusively based on the $\mathrm{sp}^{2}$-bonded hexagonal phase. Therefore, only one Gaussian peak as well as just one plasmon peak is expected in recorded spectra. According to Widmayer et al. the hexagonal phase of BN has a characteristic plasmon energy of approximately $26 \mathrm{eV}$ [139]. In addition, due to $\pi$-bonds inside $\mathrm{sp}^{2}$-bonded materials, there is also an inter-band transition $\pi \rightarrow \pi^{*}$ with an energy of about $8 \mathrm{eV}$ [139].

The XPS-measurements of the t-BN thin films were performed on energy ranges around the B- and N-peak. Again, the results were consistent regarding all investigated samples.

Typical XPS-spectra of the B- and N-peaks are shown in figure $5.3 \mathrm{a}$ and b. The corresponding energy ranges regarding the inter-band and plasmon excitations were also investigated. The results are displayed in figure $5.3 \mathrm{k}$ and $\mathrm{d}$. The $\pi \rightarrow \pi^{*}$ transition has an average value of $(9.37 \pm 0.19) \mathrm{eV}$. In particular, the $\pi \rightarrow \pi^{*}$ transition regarding the $\mathrm{N}-1$ s peak exhibits a huge error margin resulting from a very high noise 
ratio caused by the edge of the plasmon peak, an increase of secondary electrons regarding this energy range as well as an overlap with a plasmon peak resulting from the polychromatic fraction of X-rays. In addition, the plasmon energy was determined by using another Gaussian distribution for the B-1s and the N-1s peak as well as the corresponding plasmon peaks leading to an average value of $(26.64 \pm 0.07) \mathrm{eV}$. As the value of $26 \mathrm{eV}$ in literature is related to a purely hexagonal structure and the turbostratic, disordered nature of the deposited thin-films might cause a slight shift in plasmon energy due to stress inside the film, this result is a strong indication for a successful growth of a hexagonal BN phase. Furthermore, the values are consistent for all grown samples at random measurements.

In addition, in order to justify the increase in ions per cycle (see section 2.5.3), the stoichiometry has been calculated based on the XPS-data of the B and N peaks.

Basically, the stoichiometry of the t-BN thin-films can be estimated by a comparison of peak intensities of the elements. To begin with, the background signal of a particular peak is subtracted. In this context, the background is assumed to exhibit a linear characteristic between initial and final point of a peak (red shaded in figure 5.4) in first order approximation. Subsequently, the intensity of the peaks, the equivalent to their integral, is determined (blue shaded). Considering an excitation energy of $1253.6 \mathrm{eV}$ from the $\mathrm{Mg}$ - $\mathrm{K} \alpha$-line of the X-ray source and the cross-sections $\sigma_{\mathrm{i}}$ of a specific element $\mathrm{i}$ [140], this leads to the following equation for the stoichiometric ratio $n_{\mathrm{A}} / n_{\mathrm{B}}$ :

$$
\frac{n_{\mathrm{A}}}{n_{\mathrm{B}}}=\frac{I_{\mathrm{A}} \cdot \sigma_{\mathrm{B}}}{I_{\mathrm{B}} \cdot \sigma_{\mathrm{A}}}
$$

The results of grown t-BN films matches the expected ratio of 1:1 for $\mathrm{B}$ and $\mathrm{N}$ with an uncertainty of approximately $0.5 \%$, averaged for all measured samples.
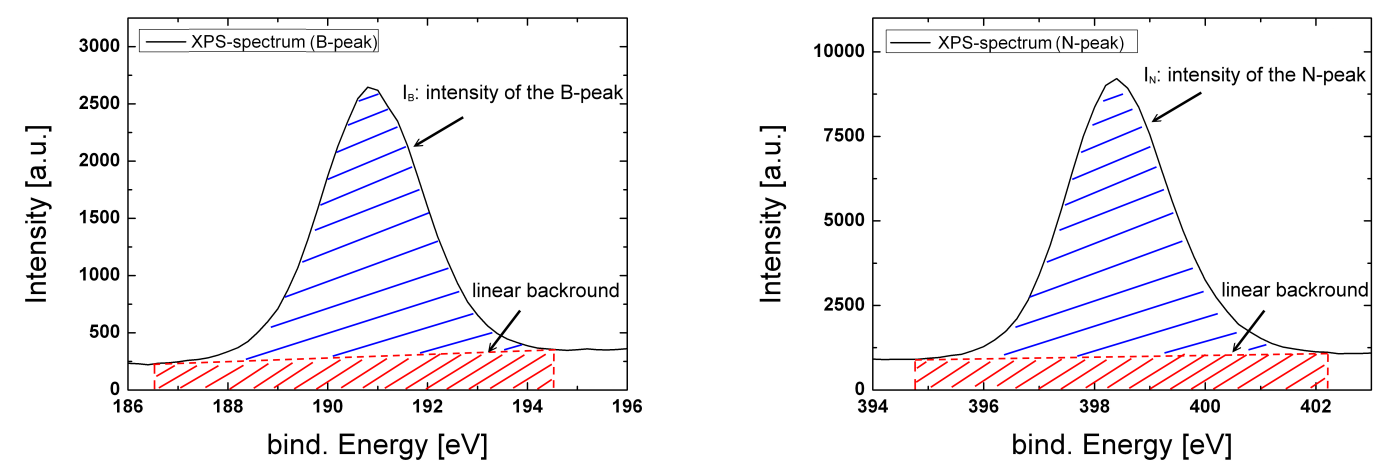

Figure 5.4: Determination of stoichiometry of a typical t-BN thin-film. The intensity of the B- (left) and N-peak (right), respectively, is represented by the blue shaded area. The linear background has been subtracted and is marked by the red shaded area. 


\subsubsection{Contaminant analysis}

Due to some maintenance and alignment issues of the ADONIS setup, some samples grown in this work between \#1235 and \#1313 were partially contaminated with a small percentage $(\leq 0.1 \%)$ of $\mathrm{Cu}$. The beam inside the ion accelerator was widened compared to the samples before. As a result, previously formed $\mathrm{Cu}$-deposits originating from several sputter experiments were re-sputtered onto the substrates during the sputter-cleaning process and therefore partially incorporated into the grown thin-films. As a result, up to one half of the samples' area was contaminated with a thin layer of copper.

In order to estimate the degree of contamination, XPS-spectra of the Cu-lines $\left({ }^{2} \mathrm{p}_{1 / 2}\right.$ and ${ }^{2} \mathrm{p}_{3 / 2}$ ) were recorded for different samples with different deposition areas and compared to each other. That way, the contamination was stopped by determining the optimal point of adjustment of the defocussing lens to a value of $-4 \mathrm{keV}$. Exemplary XPS-spectra of two ta-C samples are shown in figure 5.5. While the

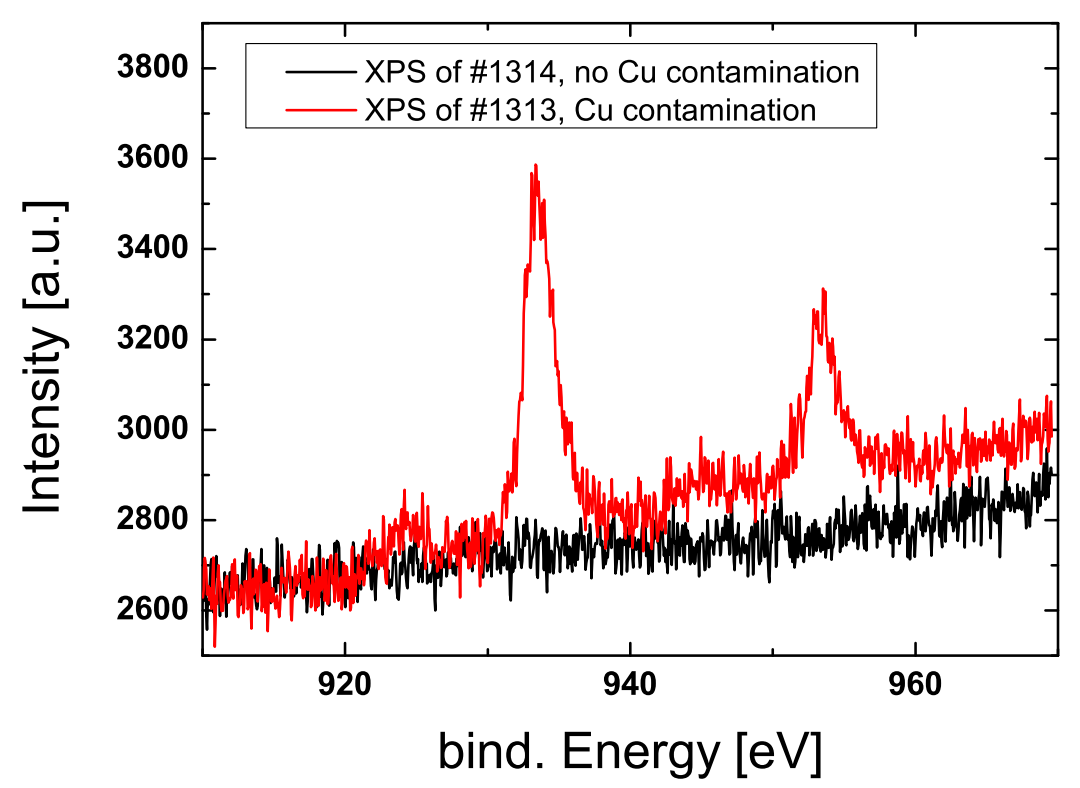

Figure 5.5: $\mathrm{Cu}$ contaminant analysis based on the comparison of two ta-C samples. While the samples with a smaller area of irradiation (e.g. \#1314) do not show any sign of $\mathrm{Cu}$, previous samples (e.g. \#1313) show pronounced Cu-lines at $932.2 \mathrm{eV}$ and $952.0 \mathrm{eV}$.

samples with a smaller area of irradiation (e.g. \#1314) do not show any sign of $\mathrm{Cu}$, previous samples (e.g. \#1313) show pronounced Cu-lines at $932.2 \mathrm{eV}$ and $952.0 \mathrm{eV}$. In this context, the same procedure was performed as for the stoichiometry of BN in section 5.2.2. The ratio of $\mathrm{Cu}$ contaminants was estimated to approximately $0.1 \%$ in the first $5 \mathrm{~nm}$ of the samples' surfaces using the intensity of the peaks as well as equation 5.1 . 
Zutz and Lyzwa showed that the majority of such a percentage of copper segregates to the surface if co-deposited during growth of a ta-C matrix [132, 141]. The impact on the conductivity of the grown thin films is not known yet and will be investigated and discussed in section 6.1.3. 


\section{$5.3 \quad$ HRTEM-measurements}

In the second part of the structural characterization, high resolution TEM measurements (HRTEM) were performed on cross-sections of all produced MASS heterostructure as well as on cross-sections of the reference heterostructures leading to detailed analysis of the micro-structure.

The preparation process of the cross-section TEM-lamellas as well as the characterization technique are described in section 4.1.2. An SEM-image of a typical cross-section TEM-lamella taken right after the thinning process using the focused ion-beam setup is shown in figure 5.6. Prior to the actual TEM-measurement, the
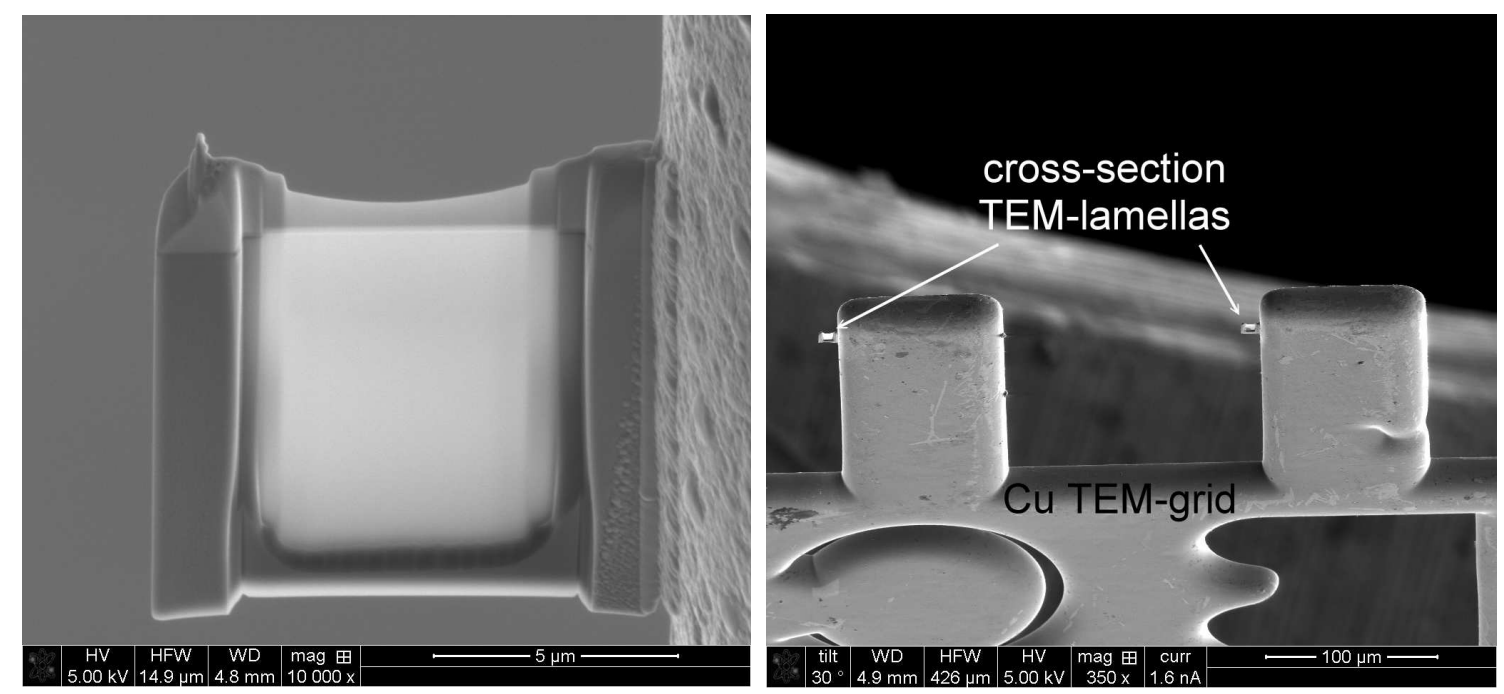

Figure 5.6: SEM-image of a typical cross-section TEM-lamellas taken right after the thinning process inside the focused ion-beam setup. The lamellas are fixed to the Cu-grid with platinum (left). Depending on the position of the lamella with respect to the $\mathrm{Cu}$-grid as well as on possible slight misalignments and deformations of the grid itself, the lamellas have to be aligned prior to the actual TEM-measurements (right).

beam path is aligned using the electrostatic lenses and corrections of the setup, especially to correct astigmatism of the images, and the position of the sample in z-direction is set to eucentric height of the setup.

In addition, the heterostructures are aligned with respect to the lattice planes of the single-crystalline substrates as this is the only defined and known part of each grown heterostructure. For the main part, this alignment process has to be performed in order to compensate for small angle deviation from the intended perpendicular view onto the cross section. These deviations are mainly caused by minor deformations of the TEM-grid and small misalignment issues during the mounting process of the lamella onto the grid during the FIB-preparation.

In this context, the sample holder can be tilted independently in $\alpha$ - and $\beta$-direction ( $\alpha \perp \beta$, both with respect to the $\mathrm{x}-\mathrm{y}$ plane). The holder is tilted until the closest pole of the crystalline structure in diffraction mode is positioned in the center of the fluorescent screen. The correction angles are typically between $-2.5^{\circ}$ and $+2.5^{\circ}$. 
During the measurements, one of the main aspects is the determination of thicknesses of the grown layers and comparison to the calculated values according to equation 2.1 in section 2.5 .3 leading to an estimate of accuracy regarding the calculations. In order to guarantee a representative investigation of the different heterostructures, TEM-measurements were performed on cross-section lamellas which were cut out of homogeneous areas located in the middle of a deposited thin-film. Furthermore, high resolution images with magnification above 150k were recorded. At these resolutions the crystalline structures including single lattice planes as well as very thin layers of a few nanometers become visible. Therefore, the structure of the grown thin-films and the interface between the grown thin-films and the particular underlying substrates can be investigated in detail. This leads to basic information about the growth process of the thin-films and indications about possible conduction mechanisms inside the different layers of the heterostructure as they are usually linked to a certain type of micro-crystalline structure (see section 3). In addition, crystalline structures can be identified and linked to the corresponding material by a systematical analysis of the lattice constants usually obtained from the spacing between adjacent lattice planes. The distance is measured with the program Digital Micrograph (version 1.71.38) from Gatan along a line perpendicular to the plane-directions. In more detail, the contrast of the image along the line is converted into an intensity spectrum as a function distance. Therefore, the distance of ten lattice planes was measured ten times and averaged afterwards in order to minimize stress induced contrast fluctuations and defocus. The obtained values can be compared to theoretical values based on the lattice constants obtained from literature. They depend on the type of crystalline structure and the corresponding lattice constants of the material as well as on orientation of the crystal itself with respect to the incident electron beam. The spacing between adjacent lattice planes in a hexagonal crystal structure can be calculated using the following equation:

$$
d_{h k l}=\frac{a}{\sqrt{\frac{4}{3}\left(h^{2}+h k+k^{2}\right)+\frac{a^{2}}{c^{2}} \cdot l^{2}}} .
$$

The parameters a and c represent the lattice constants and $h, k, l$ the Miller indices of the hexagonal structure. Due to symmetry reason, the calculation of spacing between adjacent lattice planes in a cubic structure with lattice constant a is much easier:

$$
d_{h k l}=\frac{a}{\sqrt{\left(h^{2}+k^{2}+l^{2}\right)}} .
$$

In addition, depending on the type of crystalline structure, there are further parameters of crystalline structures influencing diffraction of electrons. The arrangement of the atoms inside a particular crystalline matrix equivalent to the position of the atoms inside the corresponding unit cell leads to constructive or destructive interference of electrons scattered at the lattice planes.

The resulting object wave functions $\psi_{O}$ of a particular crystalline structure converges to spots in the back focal plane of the microscope forming diffraction patterns which 


\begin{tabular}{|c|c|c|c|c|}
\hline $\begin{array}{l}\text { crystalline } \\
\text { structure }\end{array}$ & $\begin{array}{l}\text { Bravais } \\
\text { lattice }\end{array}$ & $\begin{array}{c}\text { present } \\
\text { reflections }\end{array}$ & $\begin{array}{c}\text { absent } \\
\text { reflections }\end{array}$ & material \\
\hline simple cubic & primitive & any h,k,l & $\begin{array}{c}\text { none } \\
\end{array}$ & e.g. Po \\
\hline $\begin{array}{l}\text { body centered cubic } \\
\text { (BCC) }\end{array}$ & $\mathrm{BCC}$ & $\mathrm{h}+\mathrm{k}+\mathrm{l}=$ even & $\mathrm{h}+\mathrm{k}+\mathrm{l}=$ odd & e.g. chromium \\
\hline $\begin{array}{l}\text { face centered cubic } \\
(\mathrm{FCC})\end{array}$ & FCC & $\mathrm{h}, \mathrm{k}, \mathrm{l}$ unmixed & $\mathrm{h}, \mathrm{k}, \mathrm{l}$ mixed & $\mathrm{Al}, \mathrm{Au}, \mathrm{Ag}, \mathrm{Pt}, \mathrm{Cu}$ \\
\hline zincblende & FCC & $\begin{array}{c}\text { similar to FCC, but } \\
\text { absent if all even and } \\
\mathrm{h}+\mathrm{k}+\mathrm{l} \neq 4 \mathrm{~N}\end{array}$ & $\begin{array}{l}\mathrm{h}, \mathrm{k}, \mathrm{l} \text { mixed and } \\
\text { if all even and } \\
\mathrm{h}+\mathrm{k}+\mathrm{l} \neq 4 \mathrm{~N}\end{array}$ & diamond, silicon \\
\hline $\begin{array}{l}\text { hexagonal close-packed } \\
(\mathrm{HCP})\end{array}$ & hexagonal & $\begin{array}{c}\mathrm{h}+2 \mathrm{k}=3 \mathrm{~N} \text { with } \mathrm{l} \text { even } \\
\mathrm{h}+2 \mathrm{k}=3 \mathrm{~N} \pm 1 \text { with } \mathrm{l} \text { odd } \\
\mathrm{h}+2 \mathrm{k}=3 \mathrm{~N} \pm 1 \text { with } \mathrm{l} \text { even }\end{array}$ & $\begin{array}{c}\mathrm{h}+2 \mathrm{k}=3 \mathrm{~N} \\
\text { with } \mathrm{l} \text { odd }\end{array}$ & h-BN, $\mathrm{ZnO}$ \\
\hline
\end{tabular}

Table 5.1: Selection rules of different crystalline structures.

can be projected onto a screen. This kind of projection is equal to the intensity of the scattered beam and therefore proportional to the square of the absolute value regarding the scattering amplitude (atomic form factor)

$$
I_{d} \propto\left|F\left(\psi_{o}\right)\right|^{2}
$$

Assuming plane waves for the incident electron beam

$$
\psi_{o}=\sum_{g} A_{g} e^{2 \pi i g \underline{r}}
$$

the spots of the particular diffraction pattern are equivalent to the Fourier transformation of the incoming plane wave in mathematical terms. In Fourier-space, this is the sum of $\delta$ functions multiplied with the scattering amplitude.

$$
F\left(\psi_{o}\right)=\sum A_{g} \delta(g-q)
$$

In addition, the interference of plane waves is accompanied by a loss of phase information in this projection. In this context, the so called structure factor describes the influence of atomic arrangement on the intensity of the diffracted electron beam leading to a decrease in intensity of certain reflections. As a result, the related selection rules have to be considered regarding the resulting reflection patterns. The selection rules of common crystalline structures are summarized in table 5.1 .

Compared to the so called "conventional" specimen preparation including an incremental thinning process using Ar-ions (see Williams, Carter: chapter 10, p. 173-193 [142]), TEM-lamellas prepared in an focused ion-beam setup using Ga-ions often exhibit lower overall crystalline quality due to occasional presence of amorphous areas after the thinning-process as well as partial incorporations of Ga-ions (e.g. in the form of clusters). In addition, the thickness of the lamellas is significantly larger compared to "conventional" specimen preparation and accompanied by frequent stress as well as slight deformations inside the TEM-lamella. Therefore, direct diffraction images were not recorded during this thesis.

Nevertheless, as the real image, recorded by the CCD-camera, is an inverse Fourier 
transformation of the diffraction image in terms of mathematics additional information can be gathered regarding the crystalline structure of the particular layers of the heterostructure by performing so called Fast-Fourier Transformation (FFT) analyses on significant areas of TEM-images subsequent to their recording using again the software Digital Micrograph. The corresponding image wave functions $\psi_{i}$ are a convolution of the object wave function $\psi_{o}$ and an expansion function $P$

$$
\psi_{i}=\psi_{o} * P
$$

They are projected onto the image screen or the CCD-camera. Therefore, the intensity $I$ of the real space image corresponds to the square of the absolute value of the image wave functions

$$
I_{i} \propto\left|\psi_{i}\right|^{2} \propto\left|\psi_{o} * P\right|^{2} .
$$

In general, a Fourier transformation of the intensity distribution

$$
F\left(I_{i}\right) \propto F\left(\left|\psi_{o} * P\right|^{2}\right)
$$

is not equal to equation 5.6 except one special condition. The equality of equation 5.6 and 5.9 is only valid if the FT of the intensity spectrum is equal to a sum of $\delta$ functions equivalent to the diffraction pattern. This condition is only met for a crystalline structure and corresponding lattice planes. Amorphous structures, on the other hand, will lead to homogeneous circular patterns if there are no crystallites with predominant orientation present in the surrounding matrix.

In general, Fast-Fourier Transformations are also frequently used to determine the lattice parameters of crystalline structures and typically performed on large area segments of recorded images leading to average values of the lattice parameters. Unfortunately, most of the investigated areas, especially inside the t-BN structure, are too small to obtain suitable results. Therefore, only the less accurate method, described earlier in this section, was used. In a final step, EDX measurements were performed but only on some ta-C based MASS diode structures as the B-signal cannot be reasonably detected by this setup.

The XPS-data in sections 5.2 .1 and 5.2 .2 indicate a successful growth of boronnitride based on a hexagonal structure as well as tetrahedral amorphous carbon. Therefore, the resulting heterostructures on $\mathrm{ZnO}$, n-type as well as p-type $\mathrm{Si}$ and $6 \mathrm{H}-\mathrm{SiC}$ are investigated in detail by TEM-measurements in the following sections. The different combinations of investigated heterostructures are summarized in table 5.2 .

\begin{tabular}{|c||c|c|c|c|}
\hline compound & ZnO & $\mathbf{n}^{+}$-Si & p-Si & 6H-SiC \\
\hline \hline t-BN & + & + & + & + \\
\hline ta-C & - & + & + & + \\
\hline
\end{tabular}

Table 5.2: Overview of investigated MASS heterostructures using TEM. All of them were successfully fabricated. 


\subsection{1 t-BN $/ \mathrm{ZnO}$ heterostructures}

During this thesis, TEM-measurements were first performed on heterostructures based on boron-nitride grown on zinc oxide. Corresponding images of a typical $\mathrm{BN} / \mathrm{ZnO}$ heterostructure are shown in figure 5.7. First of all, the images show a continuous boron-nitride layer with slight variation of thickness and low surface roughness of the underlying zinc oxide substrate. The BN thin-film is made up of only a single, hexagonal phase of $\mathrm{BN}$ and exhibits an abrupt interface to the single crystalline $\mathrm{ZnO}$-substrate indicating a direct thin-film growth without the formation of a significant interlayer.

The hexagonal lattice planes of the grown BN thin-film are oriented perpendicular to the $\mathrm{ZnO}$ substrate surface (figure 5.7b). Furthermore, the thin-film exhibits a high density of dislocations inside the hexagonal matrix (marked white in 5.7k). In order to identify the different structures as well as their orientation, fast Fourier transformations (FFTs) of certain areas corresponding to the investigated structures (squares in figure 5.7b) were performed. The results are shown in figure 5.8, The performed FFT corresponding to the $\mathrm{ZnO}$ in figure 5.8 (black square) shows anticipated $\{1010\}-,\{1011\}-,\{0001\}$ - and $\{0002\}$-reflections of the [21110]-pole which can be linked to the [0002] orientation of the visible lattice planes. The large-area FFT of the hexagonal BN-planes in figure 5.8 b shows a textured ring with twofold symmetry which is typically observed for the turbostratic phase of boron-nitride. If a FFT is performed of a very small area of the thin film (small white square in figure 5.7b), additional reflections will become more clearly visible indicating the presence of a "real" hexagonal phase in very small parts of thin-film (see figure 5.8c).

Another way to identify the particular structures is the precise measurement of the lattice spacing and lattice constants. Since both materials exhibit a hexagonal structure, theoretical calculations were performed according to equation 5.2 and compared to measurements performed with Digital Micrograph as mentioned in the section above. In order to provide reproducibility, the distance of ten lattice planes was measured ten times at different positions of the TEM-lamella for each material of the heterostructure and averaged afterwards.

As for the (0002) planes of $\mathrm{ZnO}$ this leads to an experimental value in the range of $d_{0002}=0.254(5) \mathrm{nm}$. This result is in good agreement with the calculated value of $d_{0002}=0.261 \mathrm{~nm}$. Regarding the turbostratic boron nitride thin-film, the spacing of the lattice planes was determined to a value of $d_{0002}=0.341(11) \mathrm{nm}$. This result is slightly higher than the calculated value of $d_{0002}=0.333 \mathrm{~nm}$ for a perfect h-BN crystal. The increase in the lattice constant of the grown t-BN thin-films compared to h-BN is likely caused by the high density of defects and displacements leading to a slight expansion and a bending of the crystalline structure which results in an increase of distance between lattice planes.

In another step of the structural characterization, the thickness of the grown t-BNlayer can be measured by using the Digital Micrograph software. In the present example, the determined value of the thin-film thickness of 130(2) nm is much smaller $(\approx 30 \%)$ than the calculated value using equation 2.1 of $183.5 \mathrm{~nm}$. A possible 

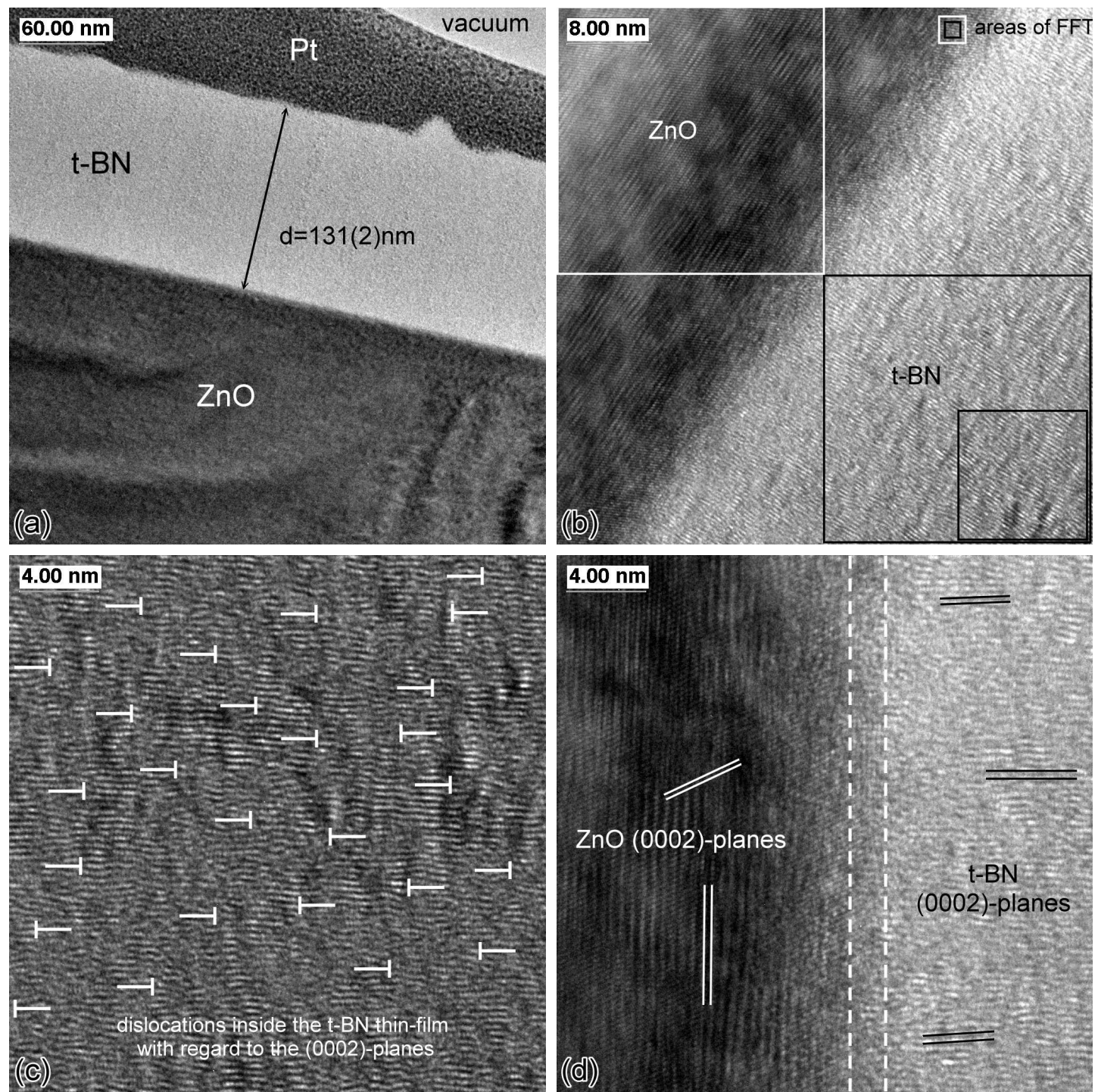

Figure 5.7: TEM-images of a t-BN/ZnO heterostructure. (a) The heterostructure exhibits a continuous BN-layer and a low surface roughness of the underlying zinc oxide substrate. (b) The $\mathrm{BN}$ thin-film is made up of only a single, hexagonal phase of BN. The hexagonal lattice planes of the grown thin-film are oriented perpendicular to the $\mathrm{ZnO}$ substrate surface. Fast Fourier transformations (FFTs) were performed on certain areas (marked by squares) in order to identify the different structures as well as the corresponding orientation. They are shown in figure 5.8. (c) The thin-film also exhibits a high density of dislocations (marked white) inside the hexagonal matrix. (d) The different crystalline structures are identified at the same magnification by determining the spacing of the particular lattice planes. In addition, the intermixing-layer between $\mathrm{ZnO}$ and t-BN is marked by the dashed white lines.

explanation can be derived from simulations of the deposition process using TRIM calculation:1] of the SRIM2008 software [143]. During the initial sputter-cleaning

\footnotetext{
${ }^{1}$ each simulation was performed for an irradiation 100000 ions
} 


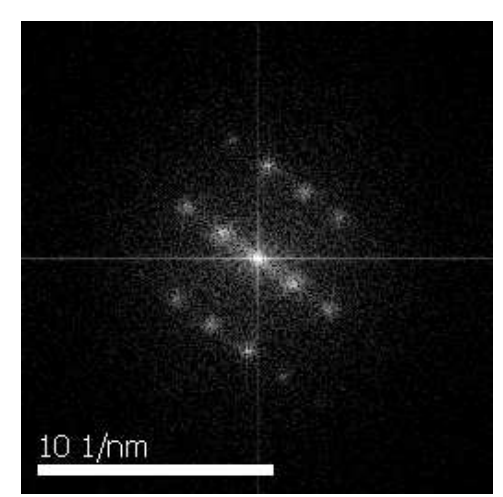

(a) FFT $\mathrm{ZnO}$

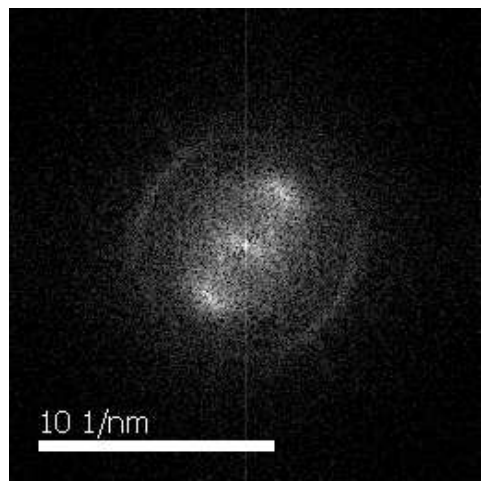

(b) FFT t-BN (large area)

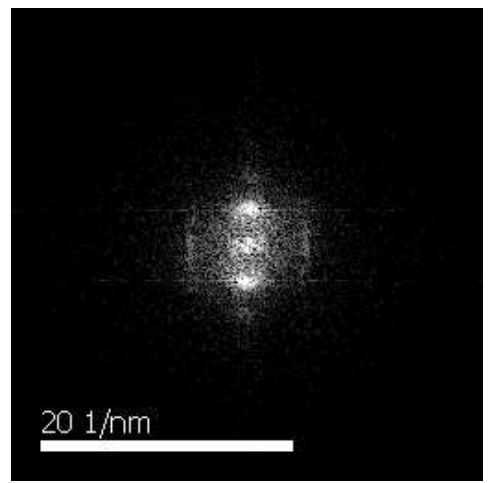

(c) FFT t-BN (small area)

Figure 5.8: FFTs of TEM-images related to a t-BN/ZnO heterostructure shown in figure 5.7b. The FFT corresponding to the ZnO structure shows $\{1010\}-,\{1011\}-,\{0001\}-$ and $\{0002\}-$ reflections of the [2 $\overline{1} \overline{1} 0]$-pole which can be linked to the (0002) planes of $\mathrm{ZnO}$ (a). A large-area FFT of the hexagonal BN-planes shows a textured ring with twofold symmetry which is typically observed for the turbostratic phase of boron-nitride. In addition, FFTs small areas recorded at high resolution show pronounced reflections of the hexagonal phase of boron-nitride (c).

process, the $\mathrm{Ar}^{+}$-ions damage the $\mathrm{ZnO}$ substrate up to a depth of about $2.5 \mathrm{~nm}$ (6.5 $\mathrm{nm} \max$ ) based on the average ion-range distribution with a straggle of approximately $2 \mathrm{~nm}$ (4.5 $\mathrm{nm} \max )$. At the same time, $\mathrm{ZnO}$ exhibits a high sputter yield of approximately 4.84 atoms/ion in total when irradiated with $\mathrm{Ar}^{+}$-ions at $1 \mathrm{keV}$ and the bigger part of the damaged area is likely constantly removed. As a side effect, the high sputter-yield, more precisely favoring zinc atoms with a ratio of 3.1:1.74 compared to oxygen, leads to a more balanced stoichiometry of the interface inside the heterostructure as the substrates are Zn-face polished [144].

At the beginning of thin-film deposition, additionally performed TRIM calculations indicate the formation of an intermixing-layer containing $\mathrm{ZnO}$ as well as implanted B- and N-atoms right at the interface of the heterostructure. The thickness of this layer would be approximately $3 \mathrm{~nm}$ based on the ion-ranges of $\mathrm{B}$ - and $\mathrm{N}$-ions at an energy of $100 \mathrm{eV}$ in $\mathrm{ZnO}$. In fact, there is a thin region of approximately $2 \mathrm{~nm}$ thickness where none of the two crystalline structures is dominant (e.g. figure $5.7 \mathrm{~d}$, dashed white lines). However, this fact is hard to prove since the layer is almost invisible, even at a magnification of 560k, and especially due to the absence of any reasonable EDX-data regarding boron (as stated above) and also nitrogen.

Assuming a successful nucleation of BN after a short time-interval, the continuous irradiation of a thin hexagonal boron-nitride layer with an alternate sequence of boron and nitrogen atoms likely causes the main part of the atomic loss during the growth process. With an estimated density of $2.25 \mathrm{~g} / \mathrm{cm}^{3}$ (h-BN) of the BN-layer, the irradiation with $\mathrm{B}^{+}$-ions at an energy of $100 \mathrm{eV}$ results in a sputter-yield of 0.283 atoms/B-ion in total, favoring $\mathrm{N}$-atoms with a ratio of 0.21:0.073. The $\mathrm{B}^{+}$ ions have a range of about $2.5 \mathrm{~nm}$ and a straggle of about $1.25 \mathrm{~nm}$. In case of the deposition of $\mathrm{N}^{+}$-ions, the sputter-yield has a value of 0.186 atoms/ $\mathrm{N}$-ion favoring 
$\mathrm{N}$-atoms with a ratio of 0.145:0.041. Range and straggle of the $\mathrm{N}^{+}$-ions are almost identical to boron due to the similar atomic mass. Both processes remove nitrogen from the nucleating layer, likely leading to a simultaneous shift of the sputtering ratio with respect to boron until an equilibrium state between $\mathrm{B}$ and $\mathrm{N}$ is reached again. Taking into account inter-mixing processes at the beginning of the deposition process, the dynamic process regarding the ratio of $\mathrm{B}$ and $\mathrm{N}$ atoms in the surface layers of the nucleating and growing thin-film as well as a sputter-yield in the range of (0.19-0.28) atoms/deposited ion, a value of $30 \%$ in loss of deposited ions is realistic.

Based on the obtained knowledge/findings, the growth process of the thin-film can be summarized as follows:

A hexagonal phase of boron nitride nucleates on top of the flat surface of the ZnO-substrate without any formation of an amorphous interlayer. The (0002) planes of the BN are oriented perpendicular to the substrate surface. Due to the ion based deposition technique, turbostratic boron-nitride is formed instead of the hexagonal phase. The difference between the calculated and the actually measured thickness of the BN thin-film is likely caused by a dynamic sputtering process accompanying the actual growth process. 


\subsection{2 $\mathrm{t}-\mathrm{BN} / \mathrm{n}-\mathrm{Si}$ heterostructures}

In a next step, silicon based heterostructures were investigated. To begin with, the micro-structure as well as the growth process of turbostratic boron-nitride reference samples based on highly n-type doped $\langle 111\rangle$ silicon substrates were analyzed. Corresponding cross-section TEM-images are illustrated in figure 5.9. The continuously grown t-BN thin-film exhibits low surface roughness as well as a smooth interface to the underlying substrate. Again, the (0002)-planes of the t-BN are aligned perpendicular to the surface of the Si-substrate and the thin-film exhibits a high dislocation density in this kind of heterostructure as well (white marks in figure $5.9 \mathrm{~d}$ ).

A major difference in comparison with the t-BN/ZnO system (see section 5.3.1) is the formation of an amorphous interlayer between the grown thin-film and the silicon substrate which is marked by the dashed lines in figure $5.9 \mathrm{a}-\mathrm{d}$. A Fast-Fourier transformation performed on this layer leads to a non-textured circular pattern indicating an entire amorphous structure. The formation of this interlayer can be explained using TRIM calculations of the sputter-cleaning process. At an energy of $1 \mathrm{keV}$ the $\mathrm{Ar}^{+}$-ions exhibit an average range of about $3.5 \mathrm{~nm}$ with a straggle of $1.5 \mathrm{~nm}$ and a maximum range of about $10 \mathrm{~nm}$. As a result, the silicon substrate is damaged up to $10 \mathrm{~nm}$ in depth.

Regarding the publication of Al-Bayati et al., low energy irradiation exceeding a fluency of about $10^{15} \mathrm{~cm}^{-2}$ causes intensive damage as well as stress and strain inside of the crystalline matrix and the structure of silicon becomes amorphous [145]. In the experiments of this thesis, this threshold is exceeded by three orders of magnitude regarding the deposited charge of $15 \mathrm{mC} \mathrm{Ar}^{+}$-ions at an energy of $1 \mathrm{keV}$. Therefore, the amorphous layer originates from the sputter-cleaning process prior to thin-film growth. The measured thickness of 9.8(6) nm (see figure [5.9a,d) is in very good agreement with the simulated data. Nevertheless, the silicon surface is smoothened and remaining contaminants as well as common thin $\mathrm{SiO}_{2}$-layers covering the Sisurface are removed in the process due to sputtering of silicon surface atoms at a sputter-yield of 0.585 atoms/ion.

Furthermore, the crystalline structures of t-BN and the $\langle 111\rangle$ silicon substrate have also been verified by additional FFTs. All of them were performed in the areas marked by squares in figure 5.9b. The FFT of the boron-nitride results in the well known pattern of a textured ring with twofold symmetry (figure 5.10k) from the section above. The FFT of the silicon lattice (figure [5.10a) shows $\{111\}-,\{131\}-$ and $\{022\}$-reflections of $[11 \overline{2}]$-pole which can be linked to the [111] orientation of the silicon substrate. The small area FFT of the Si-interlayer only leads to a circular pattern without any predominant texture indicating an amorphous structure. For additional Si related FFTs and their interpretation, see the textbook of Williams and Carter [142].

In addition, the crystalline structures were also analyzed by measuring the lattice spacings. Again, the theoretical values of t-BN structure can be calculated using equation 5.2 for a hexagonal lattice. As silicon possesses a cubic diamond crystalline structure, equation 5.3 was used. The actual measurements were performed identi- 

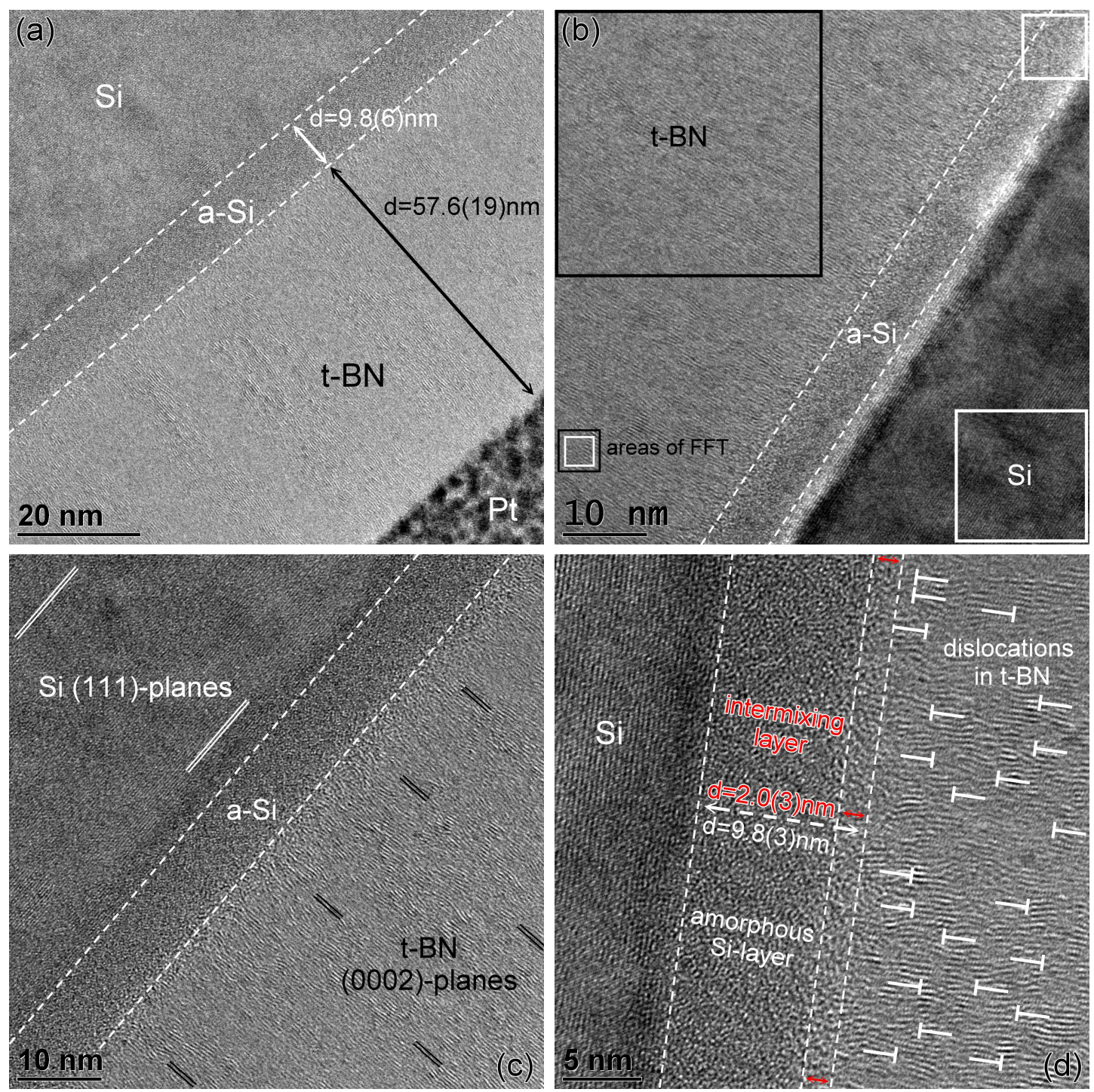

Figure 5.9: TEM-images of a t-BN/n-Si heterostructure. (a) The t-BN thin-film exhibits low surface roughness as well as a smooth interface to the underlying substrate. The heterostructure also includes an amorphous silicon interlayer between the grown thin-film and the silicon substrate (marked by the dashed lines) originating from the sputter-cleaning process prior to thin-film growth. The (0002)-planes of the t-BN are aligned perpendicular to the surface of the Si-substrate. (b) FFTs were performed on the areas marked by squares to identify the different structures. They are shown in figure 5.10. (c) The different crystalline structures are verified by measuring the spacing of the corresponding lattice planes. (d) The t-BN thin-film exhibits a high dislocation density (marked white) as well as an intermixing layer containing B, N and Si (marked red).

cally to those in section 5.3.1. Regarding the (111)-planes of the Si substrate, the experimental value of $d_{111}=0.323(8) \mathrm{nm}$ is in good agreement with the calculated value of $d_{111}=0.3135 \mathrm{~nm}$. Furthermore, the measured spacing of the t-BN (0002)lattice planes with a value of $d_{0002}=0.359(13) \mathrm{nm}$ is significantly higher than the 


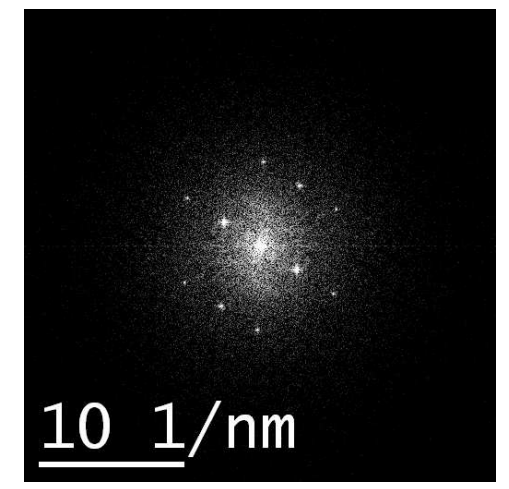

(a) FFT n-Si

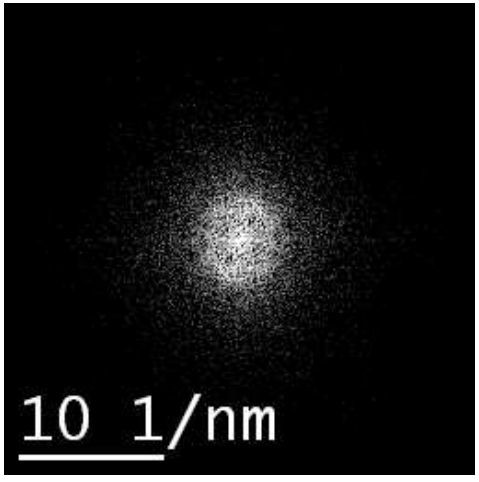

(b) FFT a-Si

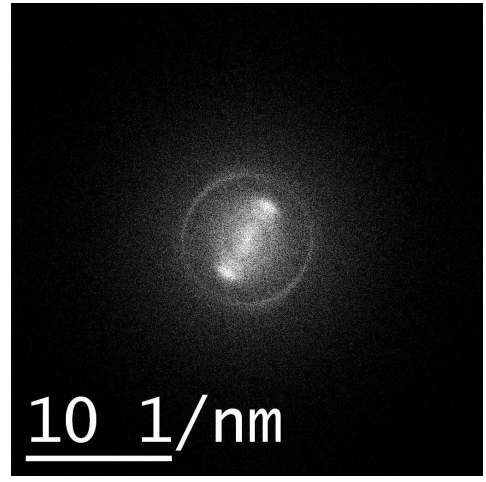

(c) FFT t-BN

Figure 5.10: FFTs of TEM-images related to a t-BN/n-Si heterostructure and performed on the marked areas in figure 5.9b. (a) The FFT corresponding to the Si structure shows $\{111\}-,\{131\}$ and $\{022\}$-reflections of the $[11 \overline{2}]$-pole which can be linked to the (111) planes of Si. (b) The small area FFT of the amorphous Si-layer leads to a circular pattern without any predominant texture indicating an amorphous structure. (c) The FFT of the hexagonal BN-planes results in the typical textured ring with twofold symmetry indicating the presence of the turbostratic phase of BN.

calculated value of $d_{0002}=0.333 \mathrm{~nm}$. Again, the increase in the measured lattice constant of the grown t-BN is caused by the high density of defects and dislocations inside the thin-films resulting in an increase of distance between lattice planes. In addition, the value is also noticeably higher than for thin-films deposited on $\mathrm{ZnO}$ but still within the error margins and therefore comparable.

In order to complete the analysis, the thickness of the grown t-BN-layer was measured by using the Digital Micrograph software. The result is quite similar to the t-BN/ZnO system as the measured value of $57.6(19) \mathrm{nm}$ is also much smaller than the calculated value of $86.6 \mathrm{~nm}$ using equation 2.1. The percentage in loss of thinfilm material is about $34.9 \%$ and therefore comparable. As the sputter-cleaning process leads to the formation of the amorphous interlayer, the loss of atoms most likely originates from the deposition process itself similar to the t-BN/ZnO system. Therefore, additional TRIM calculations based on the t-BN/Si system were performed.

At the beginning of thin-film deposition, the formation of a thin intermixing-layer containing $\mathrm{Si}$ as well as implanted B- and N-atoms right at the interface of the heterostructure is very likely. Based on an ion-energy of $100 \mathrm{eV}$, the ion-ranges of $\mathrm{B}^{+}$- and $\mathrm{N}^{+}$-ions into silicon are calculated to average values of $1.5 \mathrm{~nm}$ and $1.2 \mathrm{~nm}$ with a straggling of $0.8 \mathrm{~nm}$ and $0.7 \mathrm{~nm}$, respectively, leading to a maximal estimated thickness of around $4 \mathrm{~nm}$. At high magnifications (e.g. 560k), such a region becomes slightly visible for Si-based samples. This is exemplarily shown in figure 5.9d for an 2.0(3) nm thick layer (marked red) right at the interface between Si and BN. However, due to the lack of sufficient EDX-data regarding boron and nitrogen, evidence is just circumstantial.

Nevertheless, after a successful nucleation of BN according to the thermal-spike 
model, the growth process itself is almost identical to the t-BN/ZnO system supported by the corresponding TRIM calculations. Taking into account the dynamic nucleation and growth process as well as the calculated sputter-yields of 0.282 atoms/B-ion and 0.189 atoms/N-ion in total and adding a slightly higher ionrange during the inter-mixing process compared to the t-BN/ZnO system, a value between $30 \%$ and $35 \%$ in loss of deposited ions is a reasonable assumption matching the experimental values.

The growth process of a t-BN thin-film on silicon can be summarized in the following way:

During the sputter-cleaning process, the crystalline structure of silicon-substrate is damaged by the $\mathrm{Ar}^{+}$-ions resulting in an amorphous Si-interlayer with a thickness of up to $10 \mathrm{~nm}$. At the beginning of the deposition process, the hexagonal phase of boron nitride nucleates on top of the flat surface of the amorphous silicon layer. In this context, the roughness of the crystalline silicon is also small. The (0002) planes of the BN are oriented perpendicular to the substrate surface. Due to the ion based deposition technique, turbostratic boron-nitride is formed instead of the hexagonal phase. The difference between the calculated and the actually measured thickness of the BN thin-film is likely caused by the deposition process itself due to a dynamic sputtering process of boron and nitrogen. 


\subsection{3 ta-C/n-Si heterostructures}

In another set of reference heterostructure samples, a tetrahedral amorphous carbon thin-film was deposited onto the highly doped n-type silicon substrates instead of the turbostratic boron nitride. Corresponding images of typical ta-C/n-Si heterostructures are shown in figure 5.11.

The grown ta-C thin-film exhibits a homogeneous structure and is again separated from the Si-substrate by an amorphous Si-layer which is marked by the dashed lines in figure $5.11 \mathrm{a}$ and $\mathrm{b}$. The two amorphous structures can be differentiated by con-
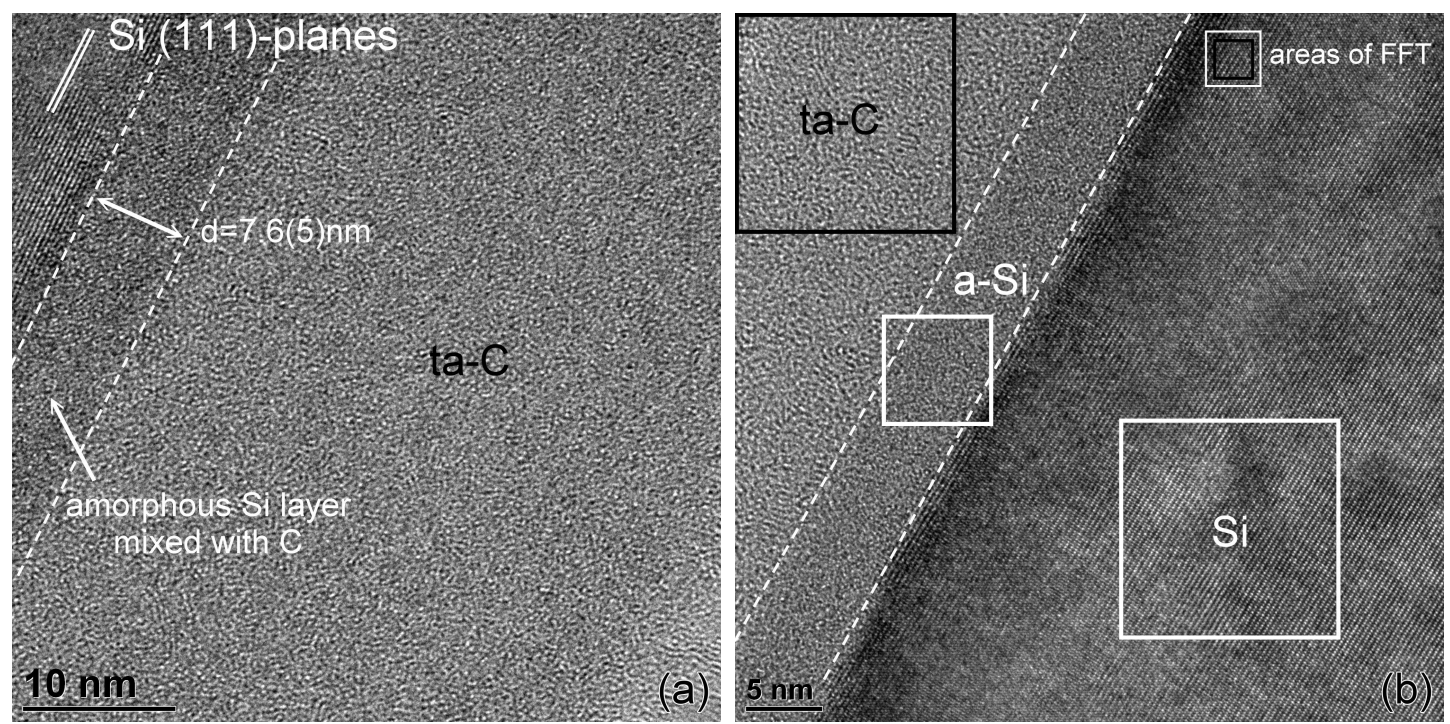

Figure 5.11: TEM-images of a ta-C/n-Si heterostructure. (a) The grown ta-C thin-film exhibits a homogeneous structure and is separated from the Si-substrate by an amorphous Si-layer which is marked by dashed lines. The two amorphous structures can be differentiated by contrast as well as tiny randomly oriented crystallites on the nanometer scale inside the matrix of the amorphous carbon thin-film. (b) The micro-structure was primarily analyzed by performing FFTs on the marked areas. The resulting patterns are illustrated in figure 5.12 .

trast as well as tiny randomly oriented crystallites on the nanometer scale inside the matrix of the amorphous carbon thin-film.

As the sputter-cleaning procedure is maintained for all utilized substrates of this work, the estimated values of dimensions regarding the amorphous Si-layer based on TRIM calculations are identical to those in section 5.3 .2 for t-BN/n-Si samples. Therefore, the measured thickness of 7.6(5) nm is in good agreement with the simulated data of the amorphous Si-layer.

Due to the amorphous character of the grown thin-film, the micro-structure was primarily analyzed by performing FFTs on the marked areas in figure 5.11 $\mathrm{b}$. The images of the corresponding patterns are illustrated in figure 5.12. The FFT of the silicon lattice (figure 5.12 a) again shows the familiar $\{111\}-,\{131\}$ - and $\{022\}$ reflections of $[11 \overline{2}]$-pole originating from the $\mathrm{Si}(111)$ planes of the substrate. In contrast, the small area FFT of the Si-interlayer possesses only a circular pattern 


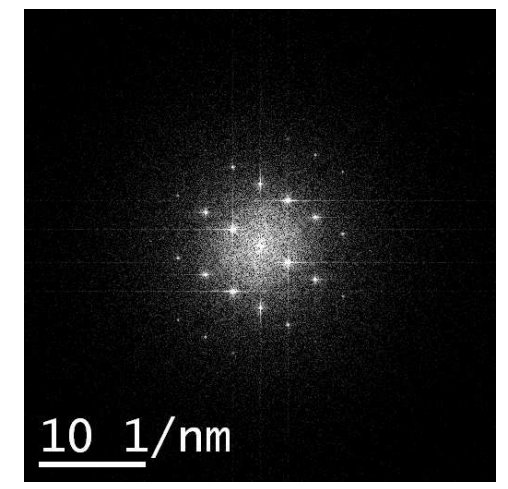

(a) FFT n-Si

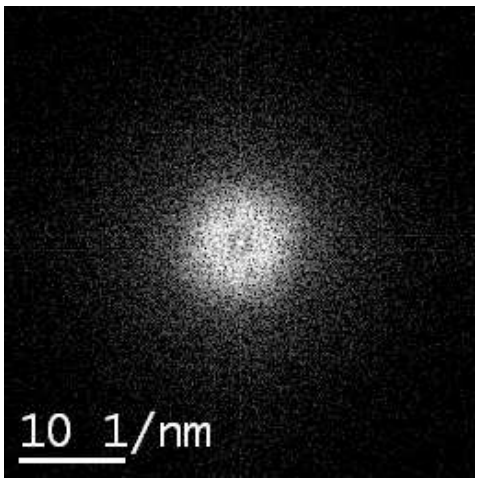

(b) FFT a-Si-C

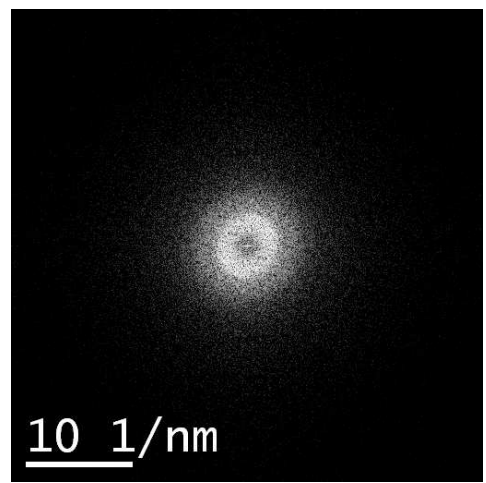

(c) FFT ta-C

Figure 5.12: FFTs of the TEM-image in figure 5.11b related to a ta-C/n-Si heterostructure. (a) The FFT of the area inside the crystalline Si possesses $\{111\}-,\{131\}$ - and $\{022\}$-reflections of the $[1 \overline{\overline{2}}]$-pole originating from the Si (111) planes of the substrate. (b) The small area FFT of the amorphous Si-layer results in a circular pattern without any predominant texture confirming the amorphous character of this volume. (c) The FFT of the grown ta-C thin-film also leads to a non-textured, but narrowed circular pattern proving the amorphous character of ta- $\mathrm{C}$ and indicate a random orientation of the nanometer scaled crystallines.

without any predominant texture confirming the amorphous character of this volume. In addition, the FFT of the grown ta-C thin-film also leads to a non-textured, but narrowed circular pattern proving the amorphous character of ta- $\mathrm{C}$ and indicate a random orientation of the nanometer scaled crystallines.

The structural analysis is completed by a detailed measurement of the lattice spacing regarding the (111)-planes of the Si-substrate as well as of the grown film-thickness. Regarding the lattice spacing of the (111)-planes of the Si substrate, the experimental value of $d_{111}=0.326(8) \mathrm{nm}$ is in good agreement with the calculated value of $d_{111}=0.3135 \mathrm{~nm}$, similar to the t-BN/n-Si system.

However, the experimentally determined values of the ta- $\mathrm{C}$ thickness do not deviate as much from the calculated values of equation 2.1 as a corresponding t-BN layer. Regarding the examples shown in this section, the determined film-thicknesses of the grown ta-C layers feature values of 38.6(5) nm (fig. 5.11a) and 20.4(3) nm (fig. $5.11 \mathrm{~b})$ matching the calculated values of $41.2 \mathrm{~nm}$ and $20.6 \mathrm{~nm}$ quite well. These results can likewise explained using the conclusions drawn from additionally performed TRIM calculations.

Starting with a flat Si surface right after the sputter-cleaning process, an irradiation with $\mathrm{C}^{+}$-ions at an energy of $100 \mathrm{eV}$ will lead to a thin region of mixed Si- and C-atoms with a maximal depth of $4.5 \mathrm{~nm}$ based on the average ion-range of $1.3 \mathrm{~nm}$ accompanied with a straggling of $0.8 \mathrm{~nm}$. Almost one third of this volume is simultaneously removed because of the sputter-yield of $0.3 \mathrm{Si}$-atoms/C-ion. Therefore, the majority of the implanted carbon atoms will accumulate at the surface-region. As a result, in combination with the continuous deposition of C-atoms, this leads to the nucleation and subsequent growth of ta- $\mathrm{C}$ according to the thermal-spike 
model. After a successful nucleation of some mono-layers of ta-C with a density of approximately $3 \mathrm{~g} / \mathrm{cm}^{3}$, the average range of the $\mathrm{C}^{+}$-ions is drastically reduced to just $0.7 \mathrm{~nm}$ with a straggle of $0.3 \mathrm{~nm}$. In the process, the sputter-yield decreases down to 0.088 atoms/C-ion of the ta-C layer.

Considering an average sputter-yield of just below $10 \%$, the formation of a thin intermixing-layer during the nucleation process as well as slight variations of filmthickness in the nm-range, an estimate of $5-10 \%$ in loss of deposited ions is reasonable.

The growth process of tetrahedral amorphous carbon (ta-C) onto a silicon substrate can be summarized as follows:

During the sputter-cleaning process, the crystalline structure of silicon-substrate is damaged by the $\mathrm{Ar}^{+}$-ions leading to the formation of an amorphous Siinterlayer with a thickness of up to $10 \mathrm{~nm}$. At the beginning of the deposition process, the tetrahedral amorphous carbon nucleates on top of the flat, but amorphous Si-surface. The slight deviation between the calculated and the actually measured thickness of the ta- $\mathrm{C}$ thin-film is mainly caused by the deposition process itself due to a dynamic sputtering process of the carbon. 


\subsection{4 t-BN/p-Si heterostructures}

In addition to the reference samples, similar heterostructures were fabricated on the basis of low doped p-type $\langle 100\rangle$ silicon substrates. Starting with samples containing turbostratic boron-nitride, images of typical t-BN/p-Si heterostructures are shown in figure 5.13. The structural properties of these heterostructures are very similar to the t-BN/n-Si system. The crystalline $\langle 100\rangle$ silicon is covered by a homogeneous layer of amorphous Si followed by the grown t-BN thin-film. The interface exhibits a small roughness and the (0002)-planes of t-BN are aligned perpendicular to the Si-surface. Furthermore, the t-BN exhibits the accompanying high dislocation density which is marked white in figure 5.13 .

Fast-Fourier transformations of all structures were performed on the areas marked with the squares in figure 5.13b and are shown in figure 5.14. The FFT of the silicon lattice (figure $5.14 \mathrm{a}$ ) shows $\{002\}-,\{1 \overline{1} 1\}$ - and $\{02 \overline{2}\}$-reflections of [011]-pole which can be linked to the [100] orientation of the silicon substrate. The small area FFT of the Si-interlayer (figure $5.14 \mathrm{~b}$ ) on the other hand possesses again only a circular pattern without any predominant texture confirming the presence of an amorphous structure. Finally, the FFT of the turbostratic boron-nitride shows the expected pattern of a textured ring with twofold symmetry (figure 5.14c). For this particular sample, the texture is very distinct and exhibits additional reflections likely resulting from a less distorted hexagonal structure.

All results up to this point indicate an almost identical growth process for the differently silicon substrates independent of their orientation. Due to the fact that corresponding TRIM calculations are just based on a statistical distribution of atoms and therefore basically on an amorphous structure inside the irradiated material, crystallographic orientation and possible corresponding channeling effects are not considered. As a result, calculated ion-ranges, straggling and sputter-yields are identical to the t-BN/n-Si system in section 5.3.2. Therefore, the thickness of 9.0(3) nm regarding the amorphous Si-layer, formed during the sputter-cleaning process, is in good agreement with the calculated value of (6.5-10) $\mathrm{nm}$.

In addition, deposited ions are also partially implanted into the amorphous silicon forming an intermixing layer. This is shown in figure 5.13. based on the loss of crystalline structure inside the BN and a maintained contrast to the homogeneous amorphous Si-layer at the same time. The thickness of this region, marked in green color, has a value of $3.1(3) \mathrm{nm}$ and matches the calculated range of $(1.5-4) \mathrm{nm}$ regarding the irradiation of Si with B- and N-ions at an energy of $100 \mathrm{eV}$.

Furthermore, the measured lattice spacing of the (100)-planes of silicon has a value of $d_{100}=0.549(18) \mathrm{nm}$ and is therefore in very good agreement with the theoretical value of $d_{100}=0.543 \mathrm{~nm}$. In the other hand, the measured spacing of the t-BN (0002)-lattice planes with a value of $d_{0002}=0.358(17) \mathrm{nm}$ is almost identical to the one measured for the t-BN/n-Si system and also significantly higher than the calculated value of $d_{0002}=0.333 \mathrm{~nm}$. Finally, the measured thickness of $66.9(3) \mathrm{nm}$ regarding the grown t-BN thin-film is approximately $32 \%$ lower than the calculated value of $97.5 \mathrm{~nm}$ which is in accordance with the previous results. 

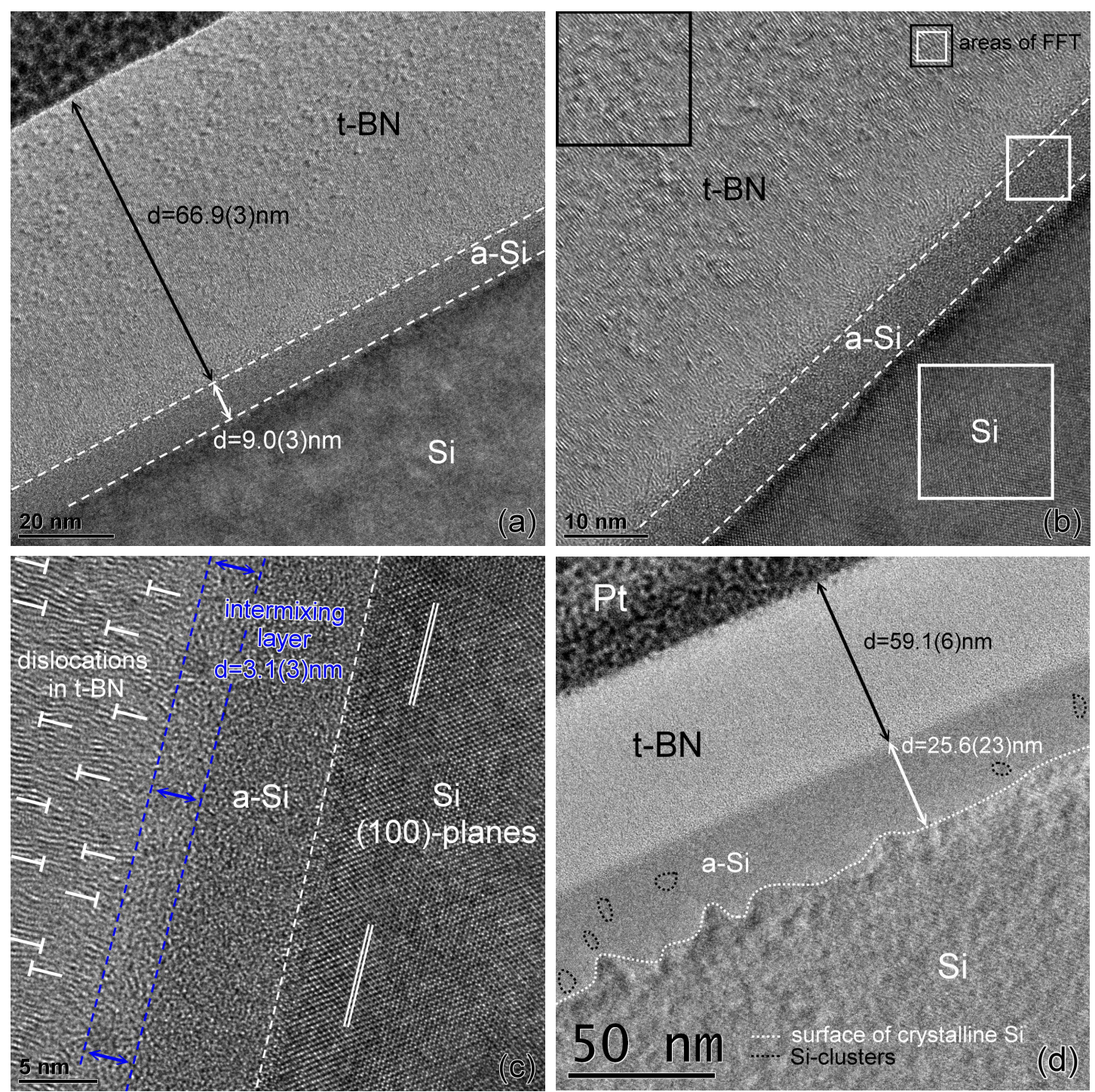

Figure 5.13: TEM-images of t-BN/p-Si heterostructures. (a) The crystalline $\langle 100\rangle$ silicon is covered by a homogeneous layer of amorphous Si (marked by dashed white lines) followed by the grown t-BN thin-film. (b) The (0002)-planes of t-BN are aligned perpendicular to the Si-surface. Fast-Fourier transformations of all structures were performed on the marked areas and are shown in figure 5.14. (c) The t-BN exhibits a high dislocation density (marked white). Furthermore, an intermixing layer is formed by partial implantation of deposited ions into the amorphous silicon (marked green). In addition, the lattice spacing of the silicon (100)-planes was measured. (d) Some samples exhibit a significantly different structure inside the interface region of the heterostructure. The amorphous Si-layer has an unusual large thickness. The surface of the crystalline silicon is rather rough and exhibits kinks with dimensions of about $5 \mathrm{~nm}$ (marked by the dotted white line). Moreover, additional crystalline Si-clusters are embedded inside the amorphous Si-layer (marked black). 


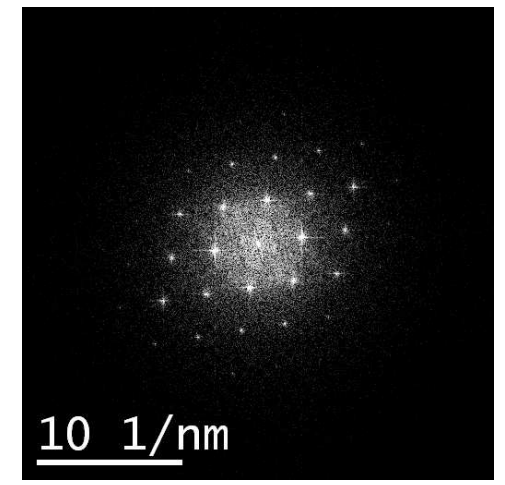

(a) FFT p-Si

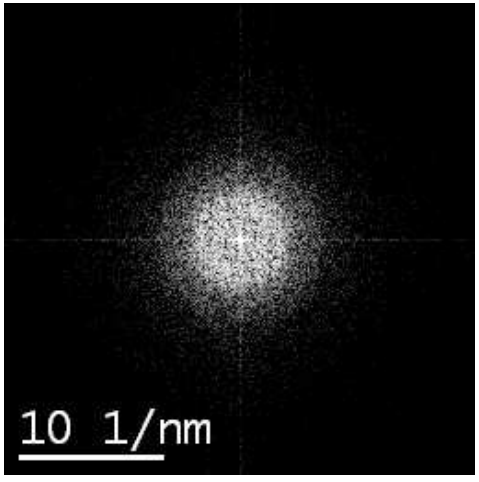

(b) FFT a-Si

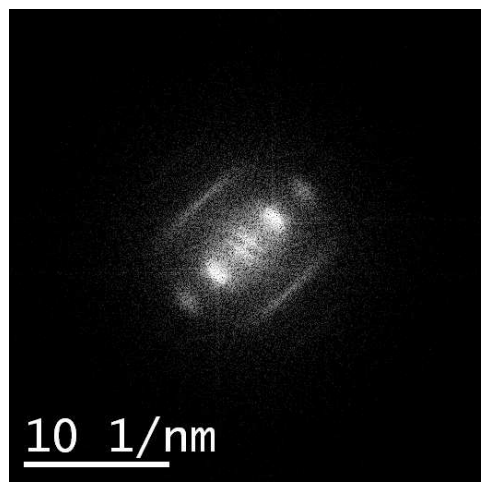

(c) FFT t-BN

Figure 5.14: FFTs of the TEM-image in figure 5.13b related to a t-BN/p-Si heterostructure. (a) The FFT of Si lattice shows $\{002\}-,\{1 \overline{1} 1\}$ - and $\{02 \overline{2}\}$-reflections of [011]-pole which can be linked to the [100] orientation of the silicon substrate. (b) The small area FFT of the amorphous Si-interlayer on the other hand possesses only a circular pattern without any predominant texture confirming the presence of an amorphous structure. (c) The FFT of the turbostratic boron-nitride shows the expected pattern of a textured ring with twofold symmetry. For this particular sample, the texture is very distinct and exhibits additional reflections likely resulting from a less distorted hexagonal structure.

In contrast to these consistent results, approximately half of the fabricated samples based on p-type doped $\langle 100\rangle$ silicon exhibit a significantly different structure inside the interface region of the heterostructure. An example is shown in figure 5.13d for another t-BN/p-Si heterostructure.

Comparing the measured thickness of 59.1(6) nm to the calculated value of $93.4 \mathrm{~nm}$, the loss of deposited ions of approximately $36 \%$ is consistent with the other BNbased heterostructures. Furthermore, the measured lattice spacings are almost identical to samples with the same type of heterostructure.

However, the amorphous Si-layer has a thickness of $25.6(23) \mathrm{nm}$ which is about $150 \%$ too high. In addition, marked by the dotted line in figure $5.13 \mathrm{~d}$, the surface of the crystalline silicon is rather rough and exhibits kinks with dimensions of about $5 \mathrm{~nm}$. Moreover, there are additional crystalline Si-clusters embedded inside the amorphous Si-layer. As this phenomenon is common for heterostructures based on p-type $\langle 100\rangle \mathrm{Si}$ in this work, it is analyzed in more detail in the following section based on the ta-C/p-Si system, particularly using EDX-measurements. 


\subsection{5 ta-C/p-Si heterostructures}

The structural analysis of silicon based heterostructures is completed by the investigation of heterostructures based on grown ta-C thin-film onto p-type $\langle 100\rangle$ silicon substrates. Corresponding images of a typical ta-C/p-Si heterostructure fabricated during this thesis is shown in figure 5.15. The grown ta-C layer possesses a homogeneous amorphous structure accompanied by a slight variation in thickness (see figure 5.15a). Furthermore, as anticipated due to the previous results, the grown thin-film is also separated from the crystalline substrate by an amorphous interlayer.

All of these structures were primarily verified due to the amorphous character of the ta-C thin-film using fast Fourier transformations of the areas marked by the squares in figure 5.15c. The results are illustrated in figure 5.16. The FFT of the silicon lattice (figure 5.16a) again shows the familiar $\{200\}-,\{1 \overline{1} 1\}$ - and $\{02 \overline{2}\}$-reflections of [011]-pole which can be linked to the [100] orientation of the silicon substrate. The FFT of the amorphous Si-interlayer (figure 5.16 b) on the other hand possesses only a circular pattern without any predominant texture confirming the assumptions based on real images. In addition, the FFT of the grown ta-C thin-film also leads to a non-textured circular pattern indicating an amorphous structure of ta-C.

The structural characterization is complemented by the precise measurement of the lattice spacing regarding the (100)-planes of the silicon substrate. In this context, the averaged experimental value of $d_{100}=0.553(8) \mathrm{nm}$ is in very good agreement with the theoretical value of $d_{100}=0.543 \mathrm{~nm}$. Despite slight variance in thickness between $61.2(3) \mathrm{nm}$ and 65.5(3) nm possibly caused by minor inhomogeneities in the deposited thin-film, the measured values are very close to the calculated value of $65.2 \mathrm{~nm}$ with a maximum discrepancy of $7.2 \%$ matching the calculated range of $5 \%-10 \%$ in loss of C-atoms when deposited onto silicon in section 5.3.3. Therefore, the measurements of ta-C grown onto p-type $\langle 100\rangle$ silicon substrates also show the anticipated results.

However, all of the few ta-C/p-Si samples which were prepared for cross-sectional TEM-measurements during this thesis exhibit a large interlayer as well as a high roughness of the crystalline silicon similar to the t-BN/p-Si heterostructures exemplarily shown in figure 5.13d of section 5.3.4. Again, there are also crystalline clusters present inside the amorphous Si-layer (marked red in figure 5.13b and d). As mentioned in section 5.3.4, the calculated values of ion-ranges, straggling and sputter-yields obtained from TRIM-calculations are identical to the counterpart on n-type silicon, which has been described in section 5.3.3. Therefore, the formation of an amorphous Si-layer with a thickness between $6.5 \mathrm{~nm}$ and $10 \mathrm{~nm}$ is expected. The corresponding area according to these calculations is marked by the dashed white lines in figures $5.13 \mathrm{~b}-\mathrm{d}$. Focusing on a typical high resolution TEM-image of the crystalline clusters at a magnification of 560k shown in figure $5.13 \mathrm{~d}$, all of them are located in a depth just exceeding the threshold range of the $\mathrm{Ar}^{+}$-ions. Additional performed FFTs of the particular clusters have been performed on the marked areas of the crystalline clusters in figure 5.15d and are shown in figure 5.17. All of these FFTs exhibit a couple of reflections identical to those originating from the [011]-pole 

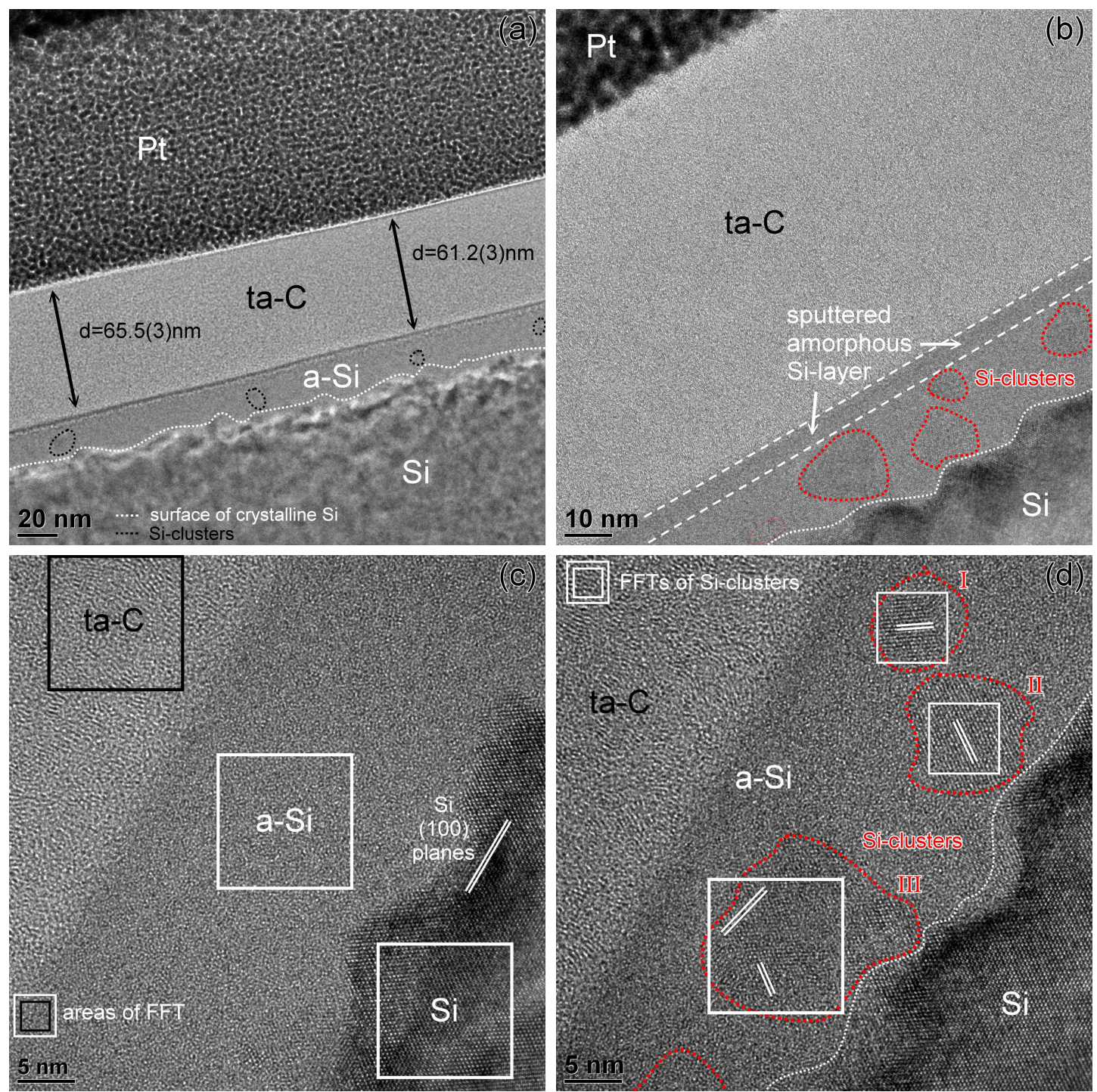

Figure 5.15: TEM-images of a ta-C/p-Si heterostructure. (a) The grown ta-C layer possesses a homogeneous amorphous structure accompanied by a slight variation in thickness and is separated from the crystalline substrate by an amorphous interlayer. The interlayer exhibits an unusual large thickness as well as a high roughness of the crystalline silicon (dotted white line) similar to some t-BN/p-Si heterostructures (see figure 5.13d) accompanied by some crystalline clusters (marked black). (b) Based on TRIM-calculations, the predicted area of the amorphous layer caused by the sputter-cleaning process is marked by dashed white lines. The numerous Si-clusters are marked red. (c) All structures were primarily verified by performing fast Fourier transformations in the marked areas. The lattice spacing regarding the (100)-planes of the silicon substrate is also measured.

(d) The crystalline Si-clusters inside the amorphous Si-layer (marked red) are investigated in detail using additional FFTs (marked by white squares) which are illustrated in figure 5.17. The orientation of the corresponding lattice planes has also been marked. 


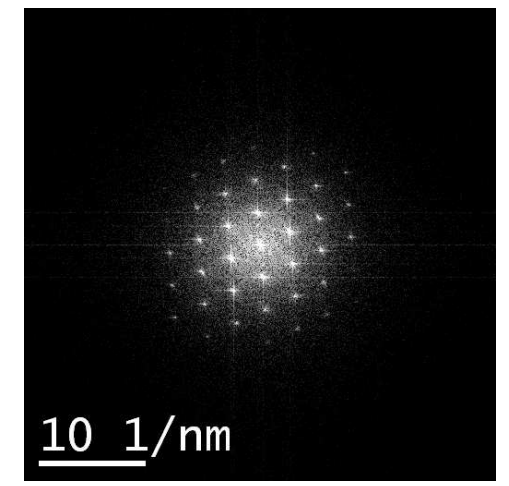

(a) FFT p-Si

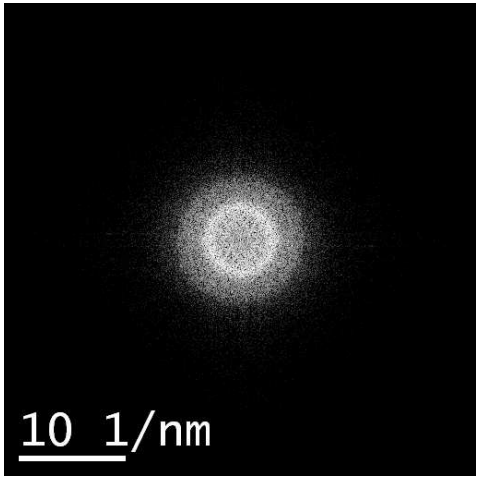

(b) FFT a-Si-C

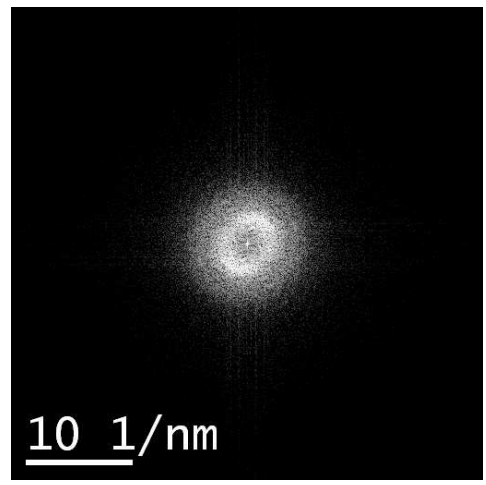

(c) FFT ta-C

Figure 5.16: FFTs of TEM-images related to a ta-C/p-Si heterostructure shown in figure 5.15. (a) The FFT of the crystalline exhibits the familiar $\{002\}-,\{1 \overline{1} 1\}$ - and $\{02 \overline{2}\}$-reflections of [011]pole which can be linked to the [100] orientation of the silicon substrate. (b) The FFT of the amorphous Si-interlayer (figure 5.16 b) possesses only a circular pattern without any predominant texture confirming the assumptions based on real images. (c) The FFT of the grown ta-C thin-film also leads to a non-textured circular pattern indicating an amorphous structure of ta-C.

the original crystalline $\langle 100\rangle$ silicon. For clarity reasons regarding the low resolution of small area FFTs, particular types of reflections have been marked:

- squares for $\{200\}$-reflections.

- circles for $\{1 \overline{1} 1\}$-reflections

- triangles for $\{02 \overline{2}\}$-reflections

In this context, cluster I exhibits reflections of the $(02 \overline{2})$ - and (1 $\overline{1} 1)$-lattice planes. Cluster II, on the other hand, exhibits reflections of the (200)- and (111)-lattice planes. Finally, cluster III exhibits all kind of reflections related to the [011]-pole of $\langle 100\rangle$ silicon. Therefore, all of these clusters are made up of pure silicon and, in addition, even match the original orientation of the wafer. The different corresponding directions of the lattice planes inside the clusters are also marked in figure $5.15 \mathrm{~d}$. Combining all of these results related to the crystalline clusters inside the amorphous interlayer with the unusual thickness, it can be concluded that all of these features likely originate from the particular wafers themselves. Beyond that, these results are backed up by several previous TEM-measurements performed during the work of Zutz on ta-C:Ni multilayer structures [132]. All of these structures were grown on top of an approximately $20 \mathrm{~nm}$ thick ta-C interlayer. The ta- $\mathrm{C}$ was deposited on sputter-cleaned p-type $\langle 100\rangle$ silicon of the same stock of wafers at identical conditions compared to the ta-C/p-Si heterostructures of this work.

From a statistical point of view, regarding all performed TEM-analyses of ta-C/p-Si interfaces over the past four years, about half of them show a flat crystalline surface of $\langle 100\rangle$ silicon covered with a thin and smooth amorphous Si-layer. In contrast, 


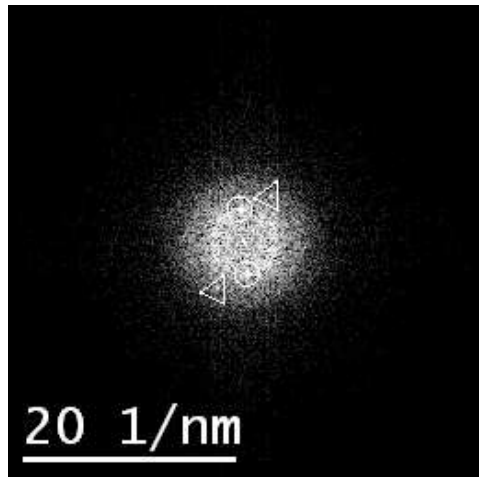

(a) FFT Si-cluster I

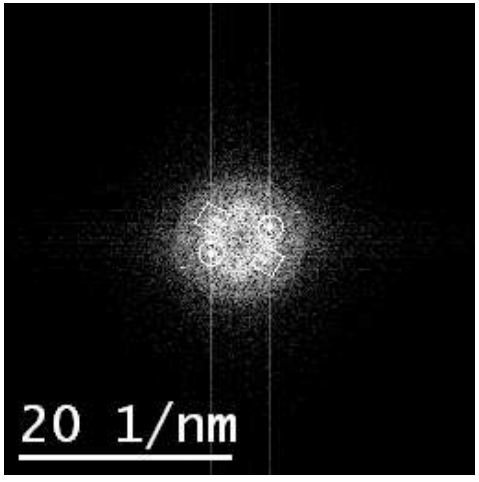

(b) FFT Si-cluster II

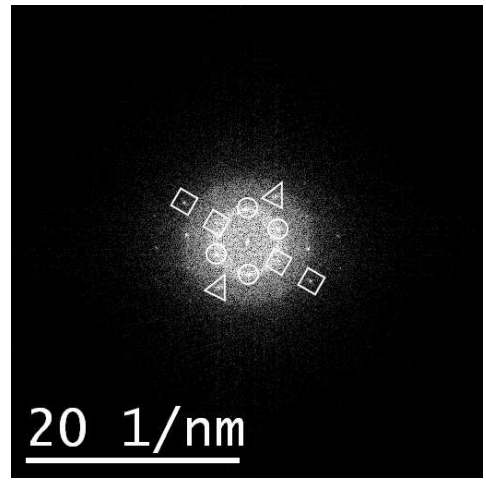

(c) FFT Si-cluster III

Figure 5.17: FFTs of crystalline clusters inside the interface region of a ta-C/p-Si heterostructure taken from the marked areas in figure 5.15d. All patterns can be linked to reflections of pure silicon. The particular reflections are marked in the following way: $\{200\}$-reflections - squares, $\{1 \overline{1} 1\}$-reflections - circles, $\{02 \overline{2}\}$-reflections - triangles.

the other half of the samples exhibit a very rough surface of the crystalline Si accompanied by a thick amorphous Si-layer and also Si-clusters in some parts of the particular lamellas. Two of these opposing samples are exemplarily shown in figure 5.18 for each type of interface structure (smooth interface: a and c, rough interface: $\mathrm{b}$ and $\mathrm{d}$ ). Therefore, the micro-structures of both particular types of interlayers are almost identical to the ones investigated during this thesis. Furthermore, the pure ta-C layer of each sample had a thickness of about 18.7(4) nm which is in good agreement with the calculated value of $20.6 \mathrm{~nm}$ for these kind of layers. As these numbers are equivalent to a loss in carbon atoms of $9.2 \%$, there is more statistics backing up the calculated value of 5-10\% using SRIM (see section 5.3.3).

In addition, EDX-linescans (see section 4.1.2) were performed on each sample along the green lines in figures $5.18 \mathrm{a}$ and $\mathrm{b}$ at a resolution of 256 points. The corresponding intensity spectra are shown in figure 5.19. As only the solid part of the lines contributes to the analysis of this work, the remaining parts of the scans (dotted lines) have been excluded. In addition, all spectra were normalized relative to their particular peak-intensity and the analysis is performed only qualitatively.

Starting with a pure ta-C film on the left side of the spectra, both samples exhibit only a gradual interface between $\mathrm{C}$ and $\mathrm{Si}$ as the silicon signal increases gradually at the expense of carbon. Due to shifts of the line-scan relative to the original position during the measurements caused by thermal sample drift and minor automatic beam-shift correction the width of the line-scan increases. As a result, the slopes of the signals become even more gradual. Therefore, the obtained range of about $10 \mathrm{~nm}$ regarding the intermixing layer of $\mathrm{C}$ and $\mathrm{Si}$ is too high, but still inside an adequate error margin for this qualitative analysis compared to the calculated value of $5 \mathrm{~nm}$ at maximum range. Finally, regarding the EDX-line-scan of the smooth sample in figure 5.18a, there are no unexpected kinks in the silicon spectrum as well as no abrupt increase of the oxygen signal in the interface region between the crystalline 

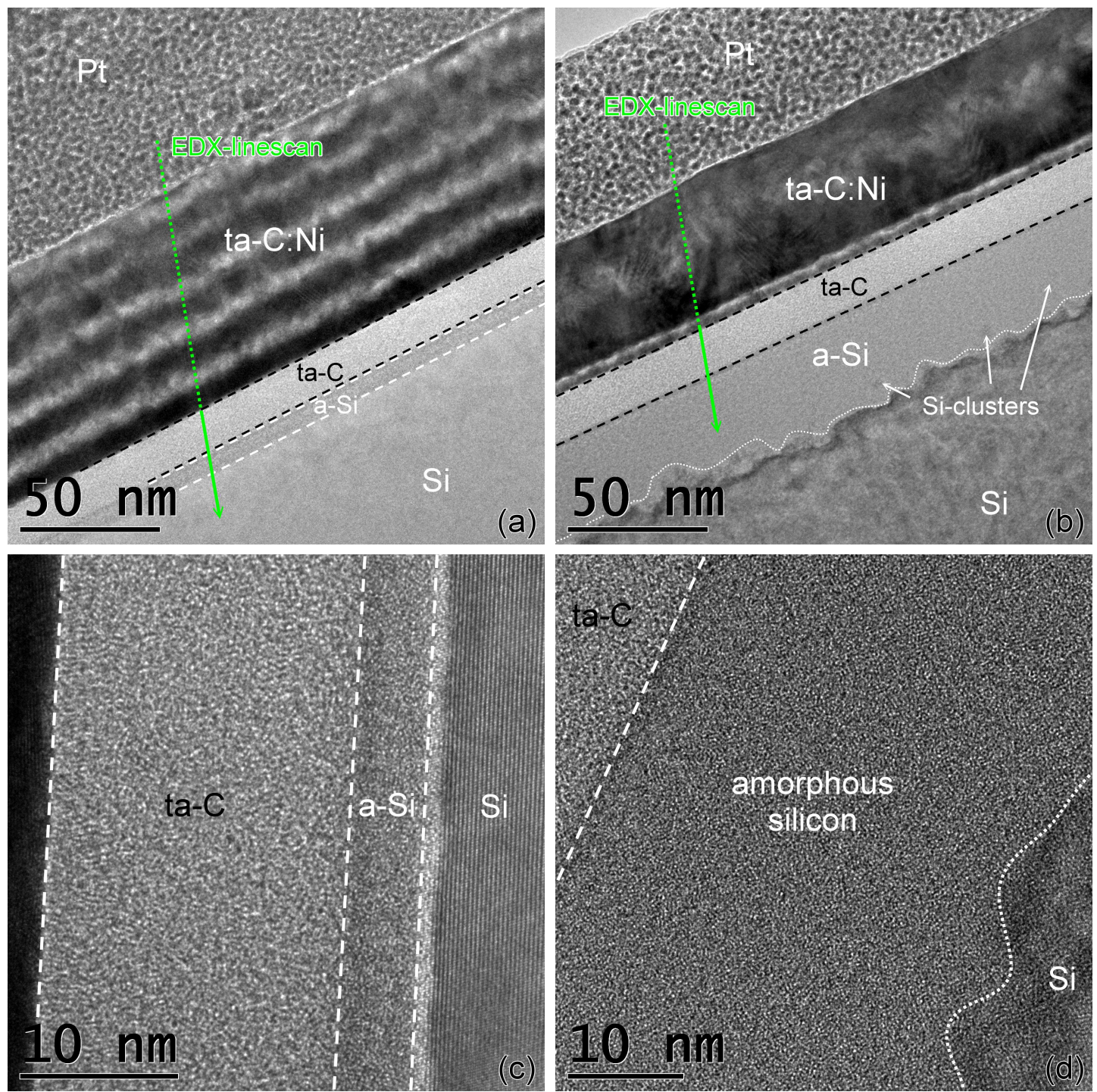

Figure 5.18: Investigation of different interface structures in the ta-C/p-Si system. According to statistics, about half of them show a flat crystalline surface of $\langle 100\rangle$ silicon covered with a thin and smooth amorphous Si-layer. The other half exhibits a very rough surface of the crystalline Si accompanied by a thick amorphous Si-layer interspersed by some Si-cluster. The smooth interface (marked by the dashed lines) is shown in (a) and (c), the rough interface (marked by the white dotted line), on the other hand, in (b) and (d). EDX-linescans were performed on each sample along the green lines. As only the solid part of the lines contributes to the analysis of this work, the remaining part of the scans (dotted lines) have been excluded. The corresponding EDX-intensity spectra are shown in figure 5.19

and the amorphous structure of silicon. In addition, illustrated in figure 5.18b, the performed line-scan of the rough sample ends right in the middle of the amorphous layer but exceeds the range of the mixing region. Again, the corresponding spectra do not show any sign of oxygen contaminants (e.g. from $\mathrm{SiO}_{2}$ ) or abrupt changes in 

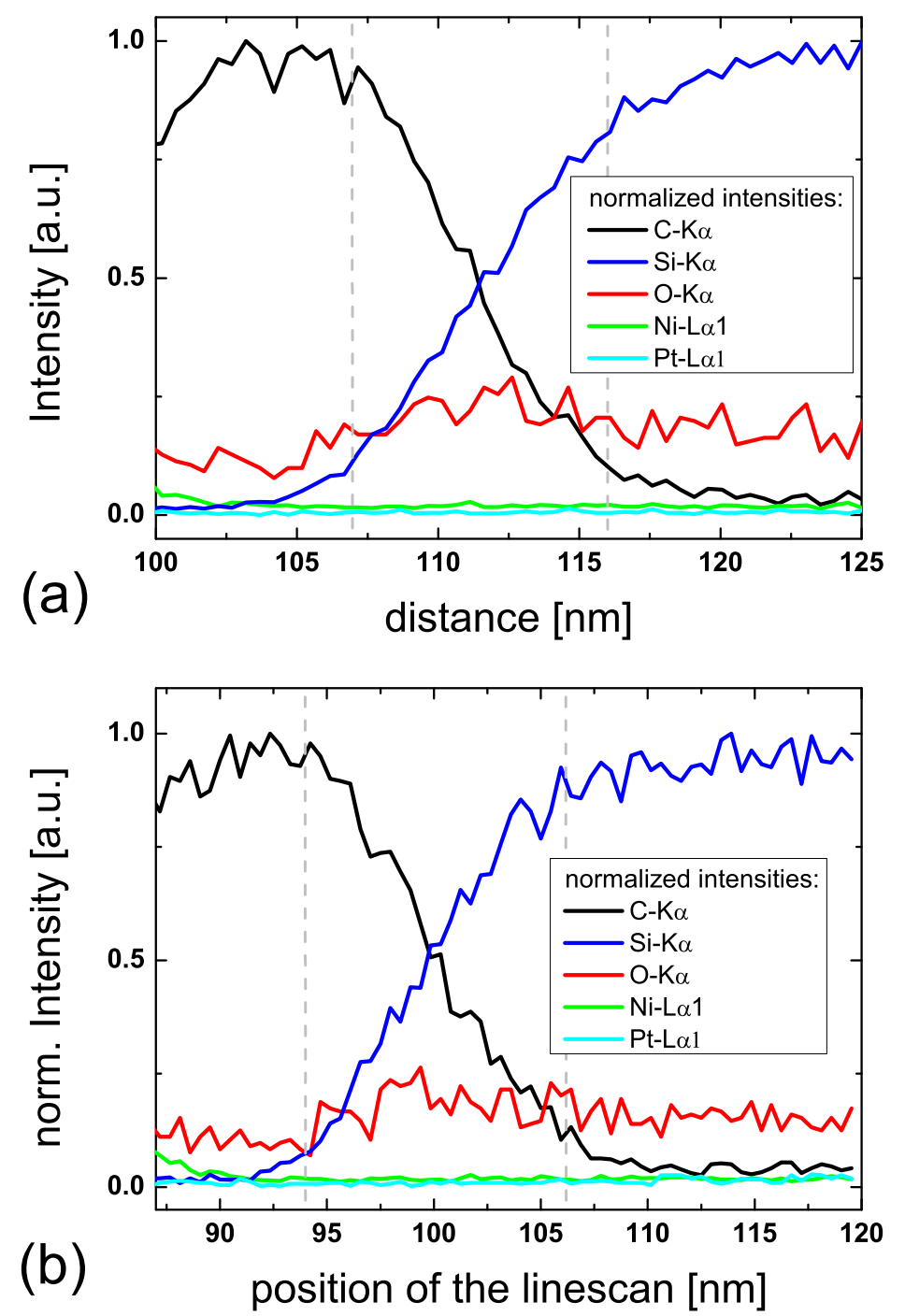

Figure 5.19: EDX-line-scans of a ta-C/p-Si heterostructure performed along the green solid lines in figure 5.18 at a resolution of 256 points. All spectra were normalized relative to their particular peak-intensity. Starting with a pure ta-C film on the left side of the spectra, both samples exhibit only a gradual interface between $\mathrm{C}$ and $\mathrm{Si}$. Therefore, the thickness of the intermixing region (gray dashed lines) of $\mathrm{C}$ and $\mathrm{Si}$ is too high when compared to TEM-measurements. (a) EDX spectra of the linescan in figure 5.18a, There are no unexpected kinks in the silicon spectrum as well as no abrupt increase of oxygen signal in the interface region between the crystalline and the amorphous structure of silicon. (b) EDX spectra of the linescan in figure 5.18b. Again, the corresponding spectra do not show any sign of oxygen contaminants or abrupt changes in the Si signal. Therefore, this layer originally contains only pure silicon.

the Si signal. Therefore, this layer originally contains only pure silicon, too.

For comparison reasons, an EDX-spectrum of a vanadium-dioxide/silicon heterostructure is shown in figure 5.20a. It was performed as part of the thesis of P. Ehrhardt 
[146]. The heterostructure exhibits a very thin $\mathrm{SiO}_{2}$-layer in the interface region
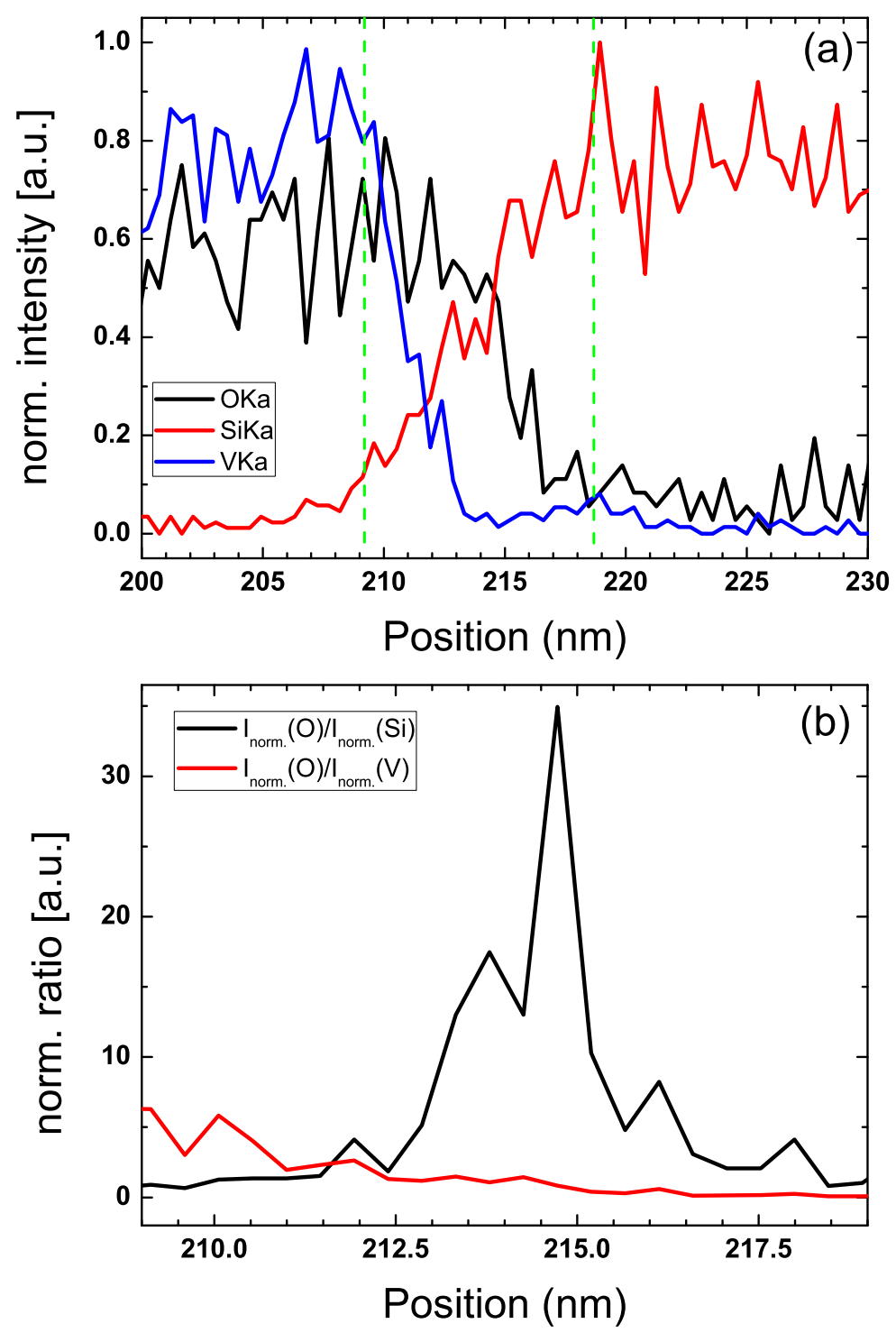

Figure 5.20: (a) EDX-line-scans of a $\mathrm{VO}_{2} / \mathrm{Si}$ heterostructure containing a $\mathrm{SiO}_{2}$-layer. The heterostructure exhibits a very thin $\mathrm{SiO}_{2}$-layer in the interface region (marked by green dashed lines) right on top of the crystalline silicon resulting in an abrupt decrease of the vanadium signal and an increase in the Si-signal while the oxygen-signal is almost constant at first and decreases slowly with increasing Si-content. (b) This can be clarified on the basis of the corresponding intensity ratios indicating the presence of a very thin layer containing Si and O.

(marked by green dashed lines) right on top of the crystalline silicon resulting in an abrupt decrease of the vanadium signal and an increase in the Si-signal while the oxygen-signal is almost constant at first and decreases slowly with increasing Si-content. This can be clarified on the basis of the corresponding intensity ratios in 
figure $5.20 \mathrm{~b}$ indicating the presence of a very thin layer containing Si and O. Thus, compared to the spectra in figure 5.19, $\mathrm{SiO}_{2}$-contaminations of the amorphous layer in the p-Si based systems of this work can be ruled out.

In addition, as the resolution of the focused ion-beam is well below the size of the (thin) amorphous layers as well as of the silicon-clusters in the nanometer range, the formation of well defined thin amorphous layers during FIB-preparation can also be excluded.

Regarding all the results at hand obtained from a variety of samples with different methods, the two interface configurations shown in the performed TEManalyses of the ta-C/p-Si system most likely originate from the original surface properties of the silicon wafers. A possible cause is the formation of an amorphous layer during the polishing process of each wafer. Due to a lack of corresponding TEM- and EDX-analyses of the identical substrates right before deposition, the proof of this statement is only based on statistics and therefore circumstantial.

In summary, the actual growth process of t-BN and ta-C onto $\langle 100\rangle$ p-type silicon is almost identical to heterostructures based on the t-BN/n-Si and ta$\mathrm{C} / \mathrm{n}$-Si systems described in sections 5.3 .2 and 5.3.3. Deviations from the anticipated structural properties of MASS heterostructures based on silicon most likely originated from the original condition of the wafer surface prior to the sputter-cleaning process. 


\subsection{6 t-BN $/ 6 \mathrm{H}-\mathrm{SiC}$ heterostructures}

The last type of heterostructures is based on single-crystalline $\langle 0001\rangle 6 \mathrm{H}-\mathrm{SiC}$ substrates. Again, starting with samples containing turbostratic boron-nitride, images of typical t-BN/SiC heterostructures are shown in figure 5.21. The structural properties are very similar to heterostructures based on t-BN and silicon which are described in sections 5.3 .2 and 5.3.4.

First of all, the crystalline $\langle 0001\rangle$ silicon-carbide exhibits a very flat surface and is covered by a homogeneous, amorphous layer made up of $\mathrm{SiC}$ with thicknesses of 5-7 nm. On top of that, the grown t-BN thin-film again exhibits a high density of dislocations which was not marked in the images of this system for clarity reasons. Nevertheless, dislocations can be well identified inside the t-BN in figure 5.21c. Furthermore, the (0002)-planes of t-BN are also aligned perpendicular to the $\mathrm{SiC}$-surface comparable to the other substrates and the interface of t-BN and $\mathrm{SiC}$ exhibits only a small roughness.

In order to verify the structures of all materials, fast-Fourier transformations were performed on the areas marked with the squares in figure $5.21 \mathrm{c}$ and are shown in figure 5.22. The FFT of the crystalline $\mathrm{SiC}$ shows $\{0006\}$ - and $\{0110\}$-reflections of the [1120]-pole, typical for $\langle 0001\rangle \mathrm{SiC}$ in $6 \mathrm{H}$-configuration. Moreover, the FFT of the boron-nitride results in the usual pattern of a textured ring with twofold symmetry (figure $5.22 \mathrm{c}$ ). The small area FFT of the SiC-interlayer (figure $5.22 \mathrm{~b}$ ) on the other hand exhibits a circular pattern without any predominant texture confirming the presence of an amorphous structure. In order to determine the origin of the amorphous interlayer in $\mathrm{SiC}$ based heterostructures, TRIM-calculations of the sputter-cleaning process were performed at first. During the irradiation of $\mathrm{SiC}$ at an energy of $1 \mathrm{keV}$, the $\mathrm{Ar}^{+}$-ions exhibit an average range of $2.5 \mathrm{~nm}$ with a straggling of $0.9 \mathrm{~nm}$ and a maximum range of about $5.5 \mathrm{~nm}$ causing damage inside the crystalline SiC-matrix of the substrate. With a value of 4.9(2)-6.9(4) nm, depending on the particular sample, the measured thickness of the amorphous layer is in very good agreement with the calculated value and can be linked to the sputter-cleaning process prior to deposition of the thin-film. During the process, remaining contaminants covering the SiC-surface are removed due to sputtering of surface atoms at a sputter-yield of 0.535 atoms/Ar-ion in total favoring Si-atoms with a ratio of 0.345:0.190 compared to carbon. As the substrates are Si-face polished, this leads to a more balanced stoichiometry of the interface inside the heterostructure similar to the $\mathrm{ZnO}$ based heterostructures in section 5.3.1. At the beginning of the deposition process, $\mathrm{B}^{+}$- and $\mathrm{N}^{+}$-ions are partially implanted into the amorphous silicon carbide forming an intermixing layer. This is illustrated and marked red in figure $5.21 \mathrm{~b}$ based on the loss of the crystalline structure inside the t-BN layer and, at the same time, a steady contrast to the amorphous SiC-layer. The thickness of this layer, regarding this particular sample, has been measured to a value of $2.5(3) \mathrm{nm}$. This is also consistent with additional TRIM calculations performed for the irradiation of $\mathrm{SiC}\left(\rho=3.21 \mathrm{~g} / \mathrm{cm}^{2}\right)$ with boron- and nitrogen-ions. At an energy of $100 \mathrm{eV}$ this leads to a calculated average ion-range of of $0.9 \mathrm{~nm}$ with a straggling of $0.4 \mathrm{~nm}$ for 

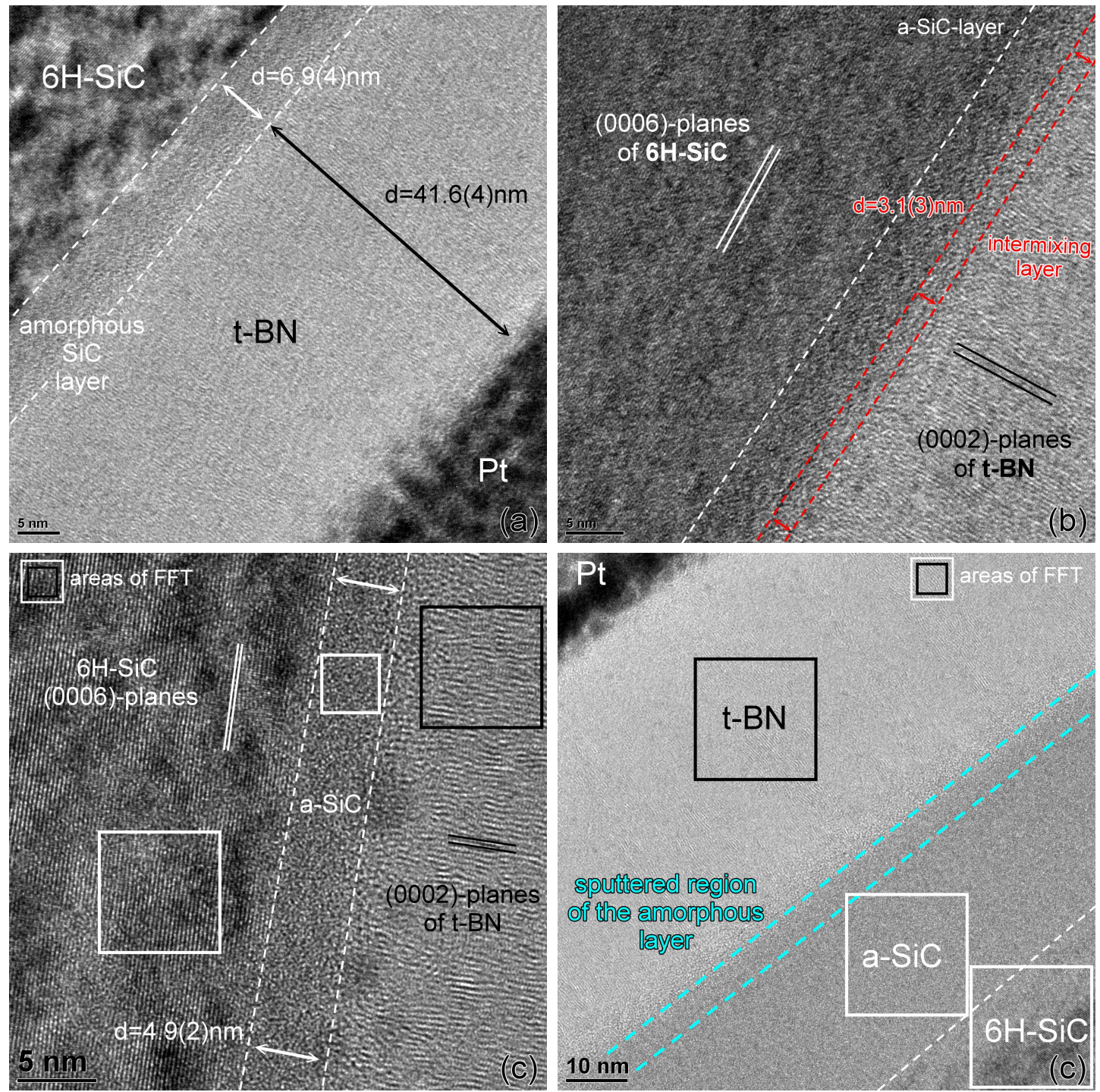

Figure 5.21: HRTEM-measurements of t-BN/SiC heterostructures. (a) The crystalline $\langle 0001\rangle$ silicon-carbide exhibits a very flat surface and is covered by a homogeneous, amorphous layer made up of $\mathrm{SiC}$ (marked by dashed white lines). The t-BN thin-film is homogeneously grown on top. (b) The (0002)-planes of t-BN are also aligned perpendicular to the SiC-surface. The interface of t-BN and SiC exhibits only a small roughness (white dashed line). An intermixing layer is formed resulting from partial implantation of $\mathrm{B}^{+}-$and $\mathrm{N}^{+}$-ions into the amorphous silicon carbide at the beginning of the deposition process (red dashed lines). Furthermore, the lattice spacings of the crystalline structures were determined. (c) In order to verify the structures of all materials, fastFourier transformations were performed on the areas marked with the squares. The corresponding images are shown in figure 5.22. (d) Some of the fabricated t-BN/SiC heterostructures exhibit an unusual thick amorphous interlayer. The measured thickness is about 5 times too high compared to common samples. The predicted amorphous region of common samplesis marked by the dashed teal lines. The different structures were identified by additionally performed FFTs in the marked areas and are shown in figure 5.23 . 


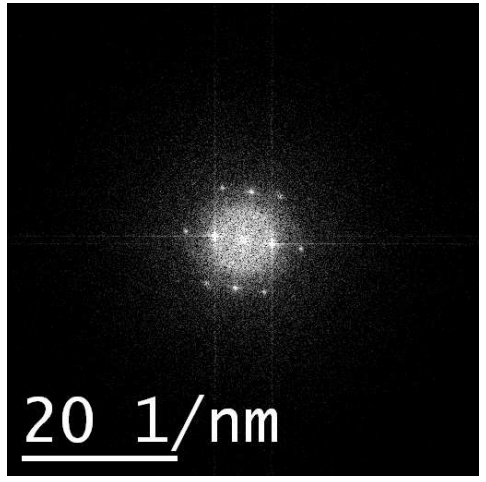

(a) $\mathrm{FFT} 6 \mathrm{H}-\mathrm{SiC}$

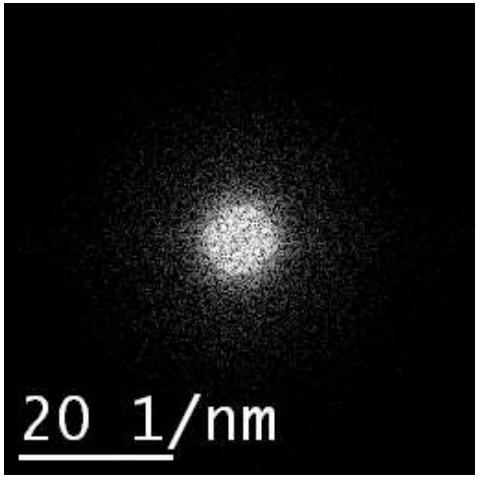

(b) FFT a-SiC

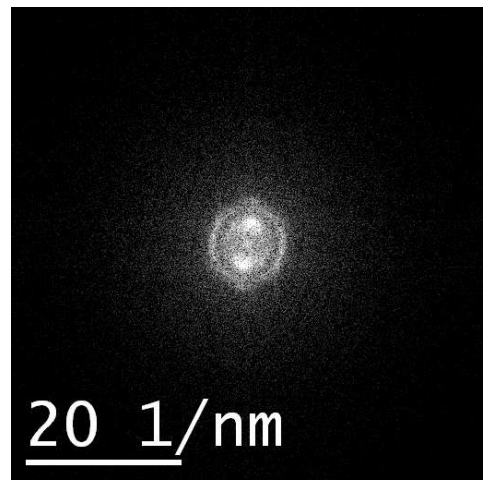

(c) FFT t-BN

Figure 5.22: FFTs of TEM-images related to a t-BN/SiC heterostructure shown in figure 5.21c (a) The FFT of the crystalline $\mathrm{SiC}$ shows $\{0006\}$ - and $\{0110\}$-reflections of the [11 $\overline{2} 0]$-pole, typical for $\langle 0001\rangle \mathrm{SiC}$ in $6 \mathrm{H}$-configuration. (b) The small area FFT of the Si-interlayer on the other hand exhibits a circular pattern without any predominant texture confirming the presence of an amorphous structure. (c) The FFT of the boron-nitride results in the usual pattern of a textured ring with twofold symmetry.

boron and $0.8 \mathrm{~nm}$ with a straggling of $0.4 \mathrm{~nm}$ for nitrogen. The maximum ranges are approximately $2 \mathrm{~nm}$ and $1.8 \mathrm{~nm}$, respectively. The corresponding sputter-yields are 0.243 atoms/B-ion (0.162:0.081) and 0.245 atoms/N-ion (0.165:0.08). The calculations of the thin-film growth after a successful nucleation of the material are identical to those of sections 5.3.1 and 5.3.2.

Furthermore, the lattice spacings of the crystalline structures were experimentally determined and compared to calculated values using equation 5.2. The lattice spacing of $\mathrm{SiC}$ (0006)-planes has a value of $d_{0006}=0.265(3) \mathrm{nm}$ and is therefore in very good agreement with the theoretical value of $d_{0006}=0.252 \mathrm{~nm}$. On the other hand, the measured spacing of the t-BN (0002)-lattice planes with a value of $d_{0002}=0.355(12) \mathrm{nm}$ is consistent with values obtained for t-BN based systems and significantly higher than the calculated value of $d_{0002}=0.333 \mathrm{~nm}$.

Finally, the measured thickness of the grown t-BN thin-films is in accordance with the previous results as the measured values of 41.6(4) $\mathrm{nm}$ and $42.2(2) \mathrm{nm}$ (figures 5.21 and c) exhibit a loss of approximately $36.2 \%$ and $35.0 \%$ in comparison to the calculated value of $65.2 \mathrm{~nm}$ for both samples.

In summary, all results are consistent with the predicted growth model. However, similar to the heterostructures based on $\langle 100\rangle$ silicon in sections 5.3.4 and 5.3.5, some of the fabricated $\mathrm{t}-\mathrm{BN} / \mathrm{SiC}$ heterostructures exhibit an unusual thick amorphous interlayer. This is exemplarily shown in figure 5.21 d. With a value of 25.0(6) nm, the measured thickness is about 5 times too high compared to the theoretical value of $5.5 \mathrm{~nm}$ at maximum. In this context, the predicted amorphous region resulting from Ar-sputtering during the sputter-cleaning process prior to deposition of the thin-film is marked by the dashed teal lines in figure $5.21 \mathrm{~d}$.

Nevertheless, regarding the remaining parts of the heterostructure, the structural 
properties are almost identical to those of regular t-BN/SiC samples. The different structures were identified by additionally performed FFTs in the marked areas of figure 5.3.6 d resulting in the anticipated patterns of the t-BN/SiC system. Again, the FFT of the crystalline $\mathrm{SiC}$ results in a pattern related to $\{0006\}$ - and $\{0110\}$-reflections of the $[11 \overline{2} 0]$-pole, indicating $\langle 0001\rangle$ oriented silicon carbide in $6 \mathrm{H}$-configuration. On the other hand, the FFT of the amorphous interlayer (figure $5.23 \mathrm{~b}$ ) exhibits only a circular pattern and does not show any signs of crystalline structures confirming the predicted amorphous characteristic of the layer. At last, the FFT of the t-BN thin-film shows a strong textured ring with twofold symmetry almost forming (0002)-reflexions and likely originating from well aligned t-BN planes. Furthermore, the measured thickness of the grown t-BN thin-film has a

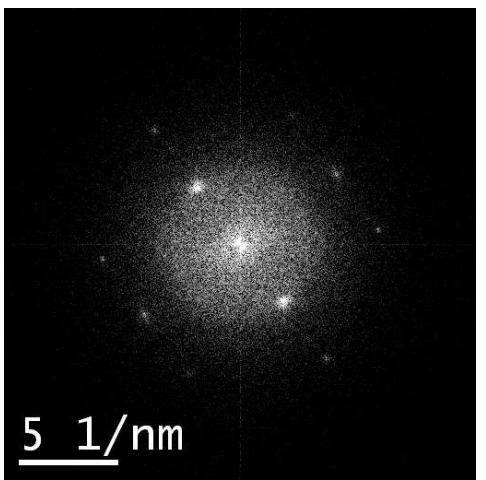

(a) FFT 6H-SiC

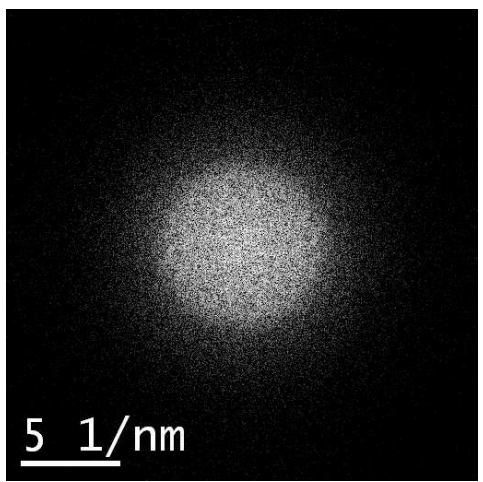

(b) FFT a-SiC

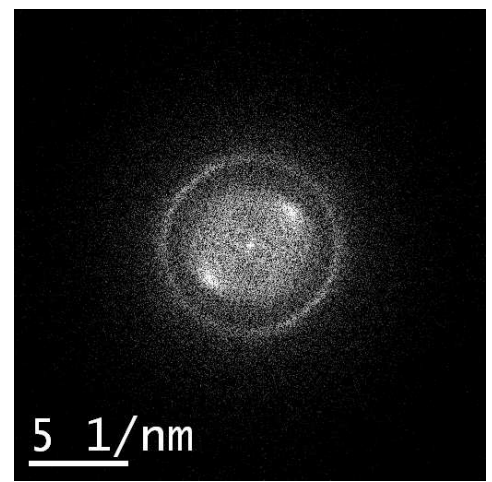

(c) FFT t-BN

Figure 5.23: FFTs of TEM-images related to a t-BN/SiC heterostructure shown in figure 5.21d, All of them lead to the anticipated patterns of the t-BN/SiC system. (a) The FFT of the crystalline $\mathrm{SiC}$ results in a pattern related to $\{0006\}$ - and $\{0110\}$-reflections of the $[11 \overline{2} 0]$-pole, indicating $\langle 0001\rangle$ oriented silicon carbide in $6 \mathrm{H}$-configuration. (b) The FFT of the amorphous interlayer exhibits only a circular pattern and does not show any signs of crystalline structures confirming the predicted amorphous characteristic of the layer. (c) At last, the FFT of the t-BN thin-film shows a strong textured ring with twofold symmetry almost forming (0002)-reflexions and likely originating from well aligned t-BN planes.

value of $55.8(4) \mathrm{nm}$ which is $31.5 \%$ below the theoretical value of $81.5 \mathrm{~nm}$ matching the estimated value of $30 \%-35 \%$ in loss of atoms in sections 5.3.1 and 5.3.2. Finally, the sputtered region of the amorphous interlayer can be identified by a faint difference in contrast between the two amorphous layers. The determined thickness of $6.5(6) \mathrm{nm}$ is quite close to the theoretical value of $5.5 \mathrm{~nm}$ at maximum.

With all these conclusive results at hand regarding the t-BN/SiC system except only one, the thick layer of the amorphous material might also be caused by the fabrication or polishing process of the original $\mathrm{SiC}$ wafers. Considering the detector properties and especially the lack of a reasonable B-signal, a detailed analysis using EDX-linescans of such a thick interlayer was performed on comparable samples based on the ta-C/ $\mathrm{SiC}$ system in section 5.3.7 in order to determine the composition of the amorphous material. 


\subsection{7 ta-C/6H-SiC heterostructures}

Finally, ta-C thin-films were also deposited onto $\langle 0001\rangle 6 \mathrm{H}$ silicon carbide substrates. Corresponding images of a typical ta- $\mathrm{C} / \mathrm{SiC}$ heterostructure are shown in figure 5.24. Similar to the t-BN/SiC system in the section right above, the grown ta-C thin-film is separated from the crystalline $\mathrm{SiC}$ substrate by an amorphous interlayer with an unusual large thickness. In the example at hand, the thickness was determined to $40.4(21) \mathrm{nm}$ which is well above the estimated value of $5.5 \mathrm{~nm}$ obtained from TRIM-calculations but comparable to the t-BN/SiC sample shown in figure $5.21 \mathrm{~d}$ in section 5.3.6. In this context, it has to be noted that all TEM-lamellas of the ta-C/SiC system prepared and analyzed during this thesis possess an interlayer of similar size. Moreover, the anticipated thickness of the amorphous region caused by Ar-ions during the sputter-cleaning process is marked by the teal dashed lines in figure $5.24 \mathrm{a}-\mathrm{c}$ for clarity reasons. In addition, the surface of the $\mathrm{SiC}$ substrate, marked by the white dotted lines in figures 5.24 and b, exhibits a high roughness accompanied by several kinks with a height of approximately $5 \mathrm{~nm}$. In contrast, the interface between the homogeneously grown ta-C and the amorphous interlayer is rather smooth. Both structures can easily be visually separated by contrast. Moreover, the strong contrast in $\mathrm{SiC}$ is likely caused by stress in the surface region of $\mathrm{SiC}$.

Based on the amorphous structure of the adjacent layers, the anticipated microstructural properties of the different materials were primarily verified using fast Fourier transformations of the areas marked by the squares in figure 5.24k and $\mathrm{d}$. The resulting patterns are shown in figure 5.25. First of all, the FFT of the crystalline $\mathrm{SiC}$ leads to the typical pattern of $\{0006\}$ - and $\{0110\}$-reflections confirming the $\langle 0001\rangle$ orientation of the substrate. In contrast, the FFT of the amorphous interlayer features only a circular pattern without any sign of texture confirming the amorphous structure inside the volume. Furthermore, in comparison to the interlayer, the FFT of the ta-C thin-film also shows a non-textured circular pattern verifying the amorphous structure but exhibits a more pronounced inner ring indicating the presence of small, randomly oriented crystallites inside the amorphous matrix on a nanometer scale. In this context, an additional small area FFT in figure 5.24k results in a faint texture of the circular pattern actually revealing signs for the existence of tiny crystallites with a predominant direction on the nanometer scale. In addition to the FFTs, the crystalline structure of the SiC-substrate was confirmed using detailed measurements of the lattice spacing. With respect to the (0006)-planes of $\mathrm{SiC}$, the measured value of $d_{0006}=0.261(9) \mathrm{nm}$ is in accordance with the theoretical one of $d_{0006}=0.252 \mathrm{~nm}$.

In a next step, a detailed EDX-analysis of the amorphous interlayer material was performed in order to determine the composition of the amorphous material. First of all, an overall EDX-spectrum of a ta-C/SiC heterostructure (STEM-image at a magnification of $480 \mathrm{k}$, approximately $400 \times 400 \mathrm{~nm}^{2}$ in area) was recorded to identify the containing elements. Such a spectrum is shown in figure 5.26. In this context, the peaks of the recorded elements are marked red. The most prominent peaks can 

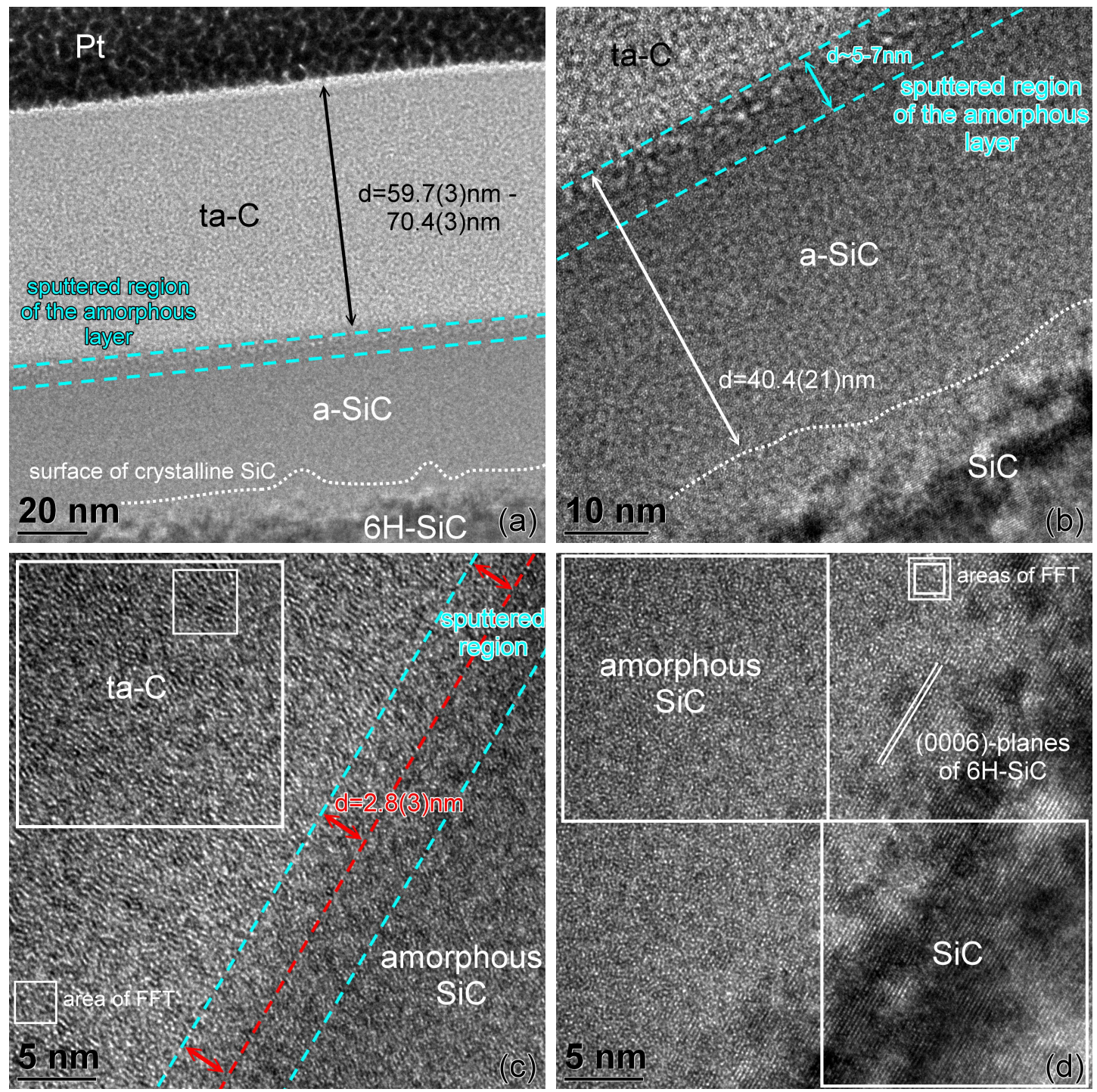

Figure 5.24: HRTEM-measurements of a ta- $\mathrm{C} / \mathrm{SiC}$ heterostructure. (a) The grown ta- $\mathrm{C}$ thin-film is separated from the crystalline $\mathrm{SiC}$ substrate by an amorphous interlayer with an unexpected large thickness. The surface of the $\mathrm{SiC}$ substrate (white dotted lines) exhibits a high roughness accompanied by several kinks with a height of approximately $5 \mathrm{~nm}$. The anticipated thickness of the amorphous region caused by sputtering of Ar-ions is marked by the teal dashed lines in all images. (b) The thickness of the amorphous interlayer has been determined. (c) Interface region between ta-C and the amorphous interlayer. FFTs were performed on the marked areas inside the ta-C-thin film and are shown in figure 5.25: and d. An intermixing layer of $\mathrm{C}$ and $\mathrm{SiC}$ can be identified based on the change in contrast with respect to the amorphous SiC-layer as well as the ta-C thin-film (red dashed line). (d) Interface region between the amorphous interlayer and the crystalline SiC-phase. Additional FFTs were performed on the marked areas of amorphous and crystalline $\mathrm{SiC}$ and are shown in figure $5.25 \mathrm{a}$ and b. The crystalline structure of the SiC-substrate was also confirmed using detailed measurements of the lattice spacing. 


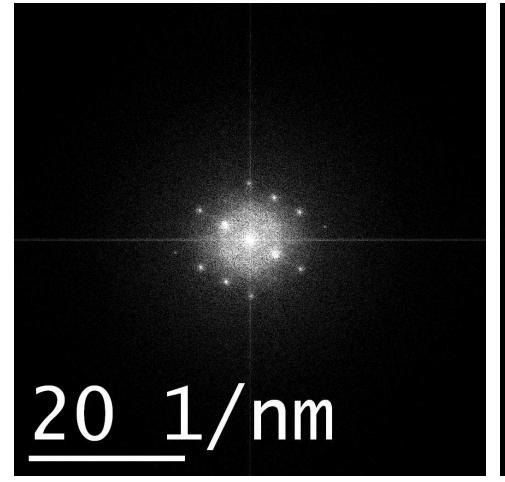

(a) $\mathrm{FFT} 6 \mathrm{H}-\mathrm{SiC}$

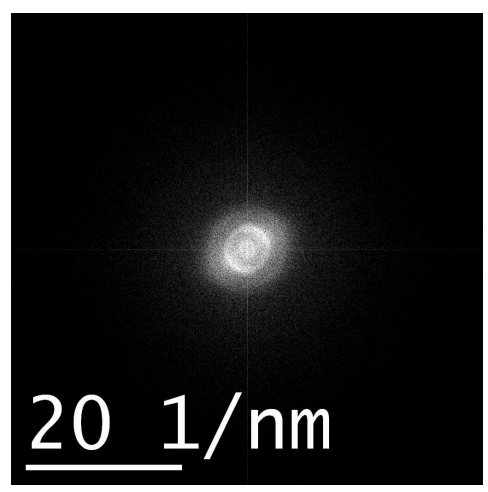

(c) FFT ta-C

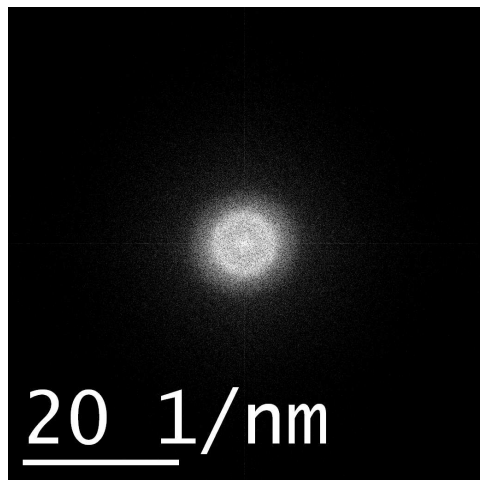

(b) FFT a-SiC

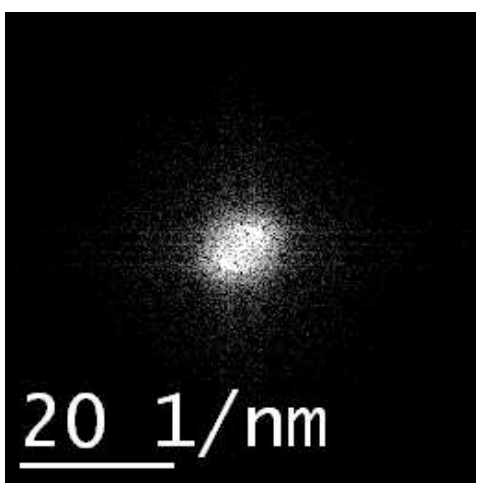

(d) FFT ta-C (small area)

Figure 5.25: FFTs of TEM-images related to a ta-C/SiC heterostructure shown in figure 5.24k and d. (a) The FFT of the crystalline SiC leads to the typical pattern of $\{0006\}$ - and $\{0110\}$ reflections of the $[11 \overline{2} 0]$-pole confirming the $\langle 0001\rangle$ orientation of the substrate. (b) The FFT of the amorphous interlayer features only a circular pattern without any sign of texture confirming the amorphous structure inside the volume. (c) The FFT of the ta-C thin-film also shows a nontextured circular pattern verifying the amorphous structure but exhibits a more pronounced inner ring indicating the presence of small, randomly oriented crystallites inside the amorphous matrix on a nanometer scale. (d) An additional small area FFT results in a faint texture of the circular pattern solidifying the assumption of the existence of tiny crystallites with a predominant direction on the nanometer scale.

be linked to carbon, silicon, platinum, copper and gallium. The carbon signal originates from the ta-C thin-film and the $\mathrm{SiC}$ as well as, in small amounts, from the precursor-gas used in the Pt-deposition during the FIB-preparation. The Pt-peak itself results solely from the deposited Pt-layer and the Si-signal is exclusively based on the SiC-substrate. The small Ga-peak is caused by the Ga-ion implanted into the lamella during cutout- and especially the thinning-processes of the FIB-preparation. The additional $\mathrm{Cu}$ signals originates from the TEM-grid which is made up of pure copper. Furthermore, the recorded spectrum does not show any sign of oxygen or other contaminants of sufficient quantity to form amorphous structures like $\mathrm{SiO}_{2}$ for example. The position of a possible oxygen peak has additionally been marked in the overall spectrum. 


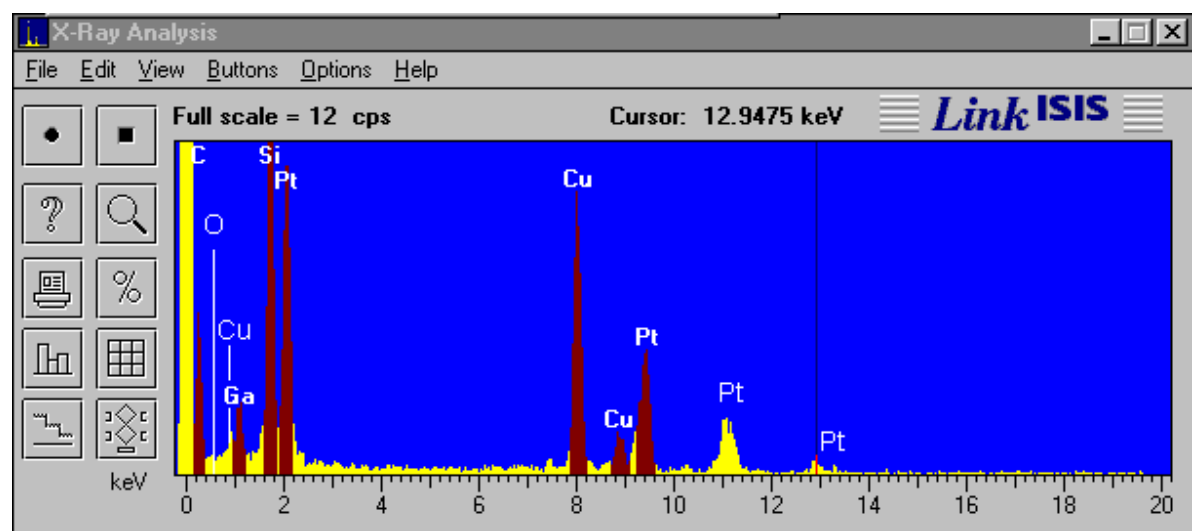

Figure 5.26: Typical overall EDX-spectrum of a ta-C/SiC heterostructure (screen shot). The most prominent peaks occurring in the spectrum can be linked to carbon, silicon, platinum, copper and gallium. The Cu-signal is based on X-rays from the TEM grid while Pt and Ga originate from the FIB-preparation process. $\mathrm{C}$ and $\mathrm{Si}$ are the only two elements of the heterostructure. In addition, there are no signs of oxygen (position marked in the spectrum) or other contaminants of sufficient quantity incorporated in the heterostructure. The peaks of recorded elements in the linescans are marked red.

Then, in order to analyze composition of the different layers regarding the ta-C/SiC heterostructure, EDX-linescans were performed along the growth-direction of the heterostructure2. In this context, only data of elements marked in figure 5.26 were recorded. The corresponding TEM-image, taken afterwards, is shown in figure $5.27 \mathrm{a}$. The linescan itself is marked green. The spectra of the different elements are illustrated in figure 5.27 b. The length of the linescan was measured using the TEM-image to calibrate the position in the EDX-spectra by dividing the total length of linescan, 206(1) nm, by its resolution of 512 points.

Each spectrum was normalized with respect to its particular maximum in order to ensure comparability of the different spectra regardless of their absolute intensities. Starting inside the Pt-layer of the lamella, the spectrum is dominated by the Ptsignal accompanied by some carbon originating from the Pt-containing precursor gas used in the FIB-preparation process. At the interface between platinum and the grown ta-C thin-film, the Pt-signal decreases abruptly to zero while the $\mathrm{C}$-signal reaches its maximum. It is maintained throughout the pure carbon-layer. After a distance of about $60 \mathrm{~nm}$ inside the ta-C layer the electron beam reaches the interface of the amorphous interlayer and the carbon signal decreases relatively slow, compared to the changes of the first interface, until an almost constant level is reached. At the same time, the intensity of silicon increases at the same rate featuring an almost inverse slope. No other element but $\mathrm{Si}$ and $\mathrm{C}$ is included in this layer indicating the presence of another $\mathrm{SiC}$ phase or $\mathrm{Si}-\mathrm{C}$ compound with an amorphous structure (a-SiC). The total width of this interface area between pure carbon (ta-C) and the amorphous $\mathrm{SiC}$ is estimated to be of about $20 \mathrm{~nm}$, marked blue in figure 5.27 a. As this interface exhibits almost twice the size compared to the first one,

\footnotetext{
${ }^{2}$ at least in the STEM-mode at a tilting-angle of $15^{\circ}$ due to the detector geometry
} 

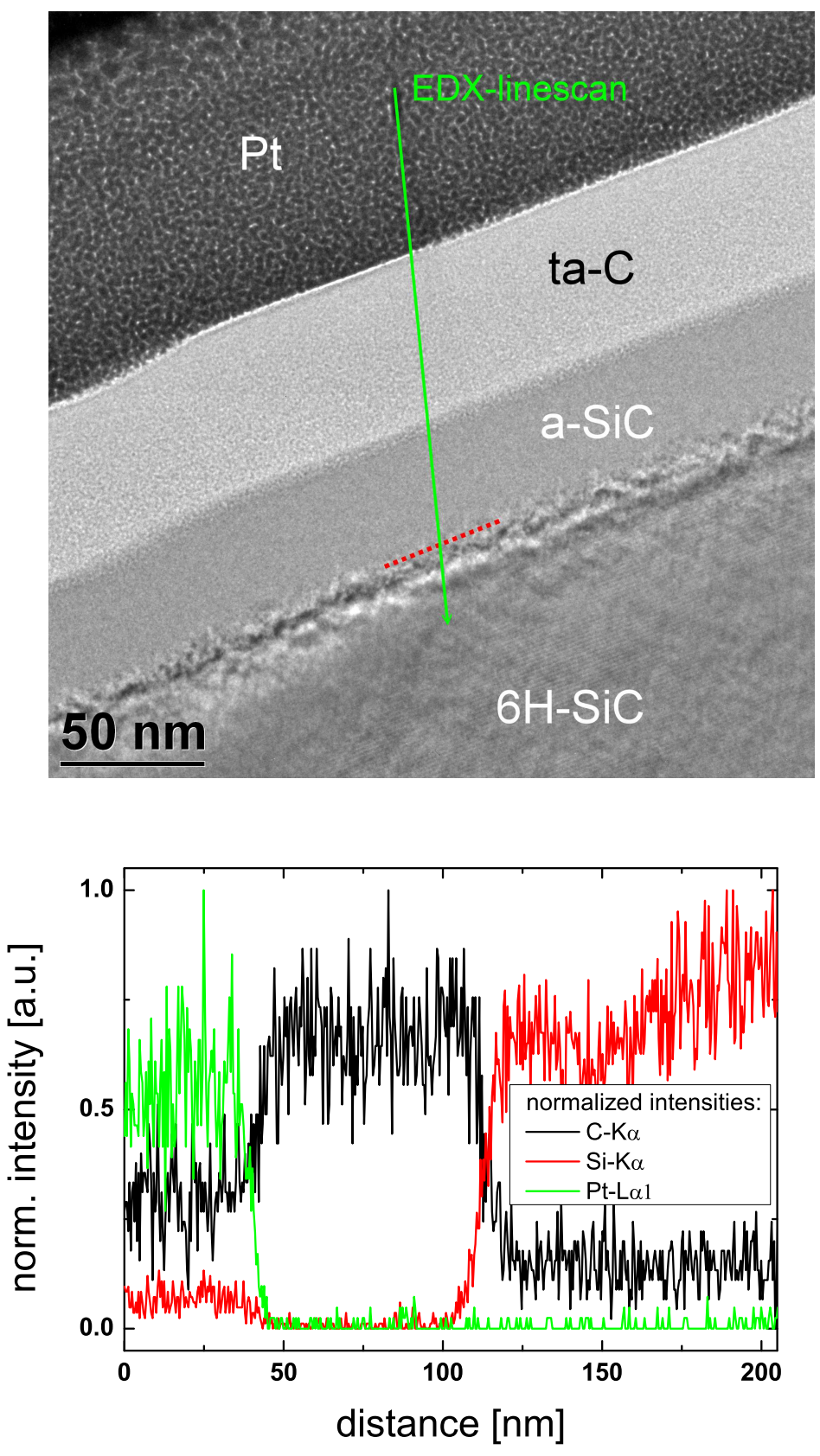

Figure 5.27: EDX-line-scan of a ta-C/SiC heterostructure. (top) The corresponding TEM-image taken right after the EDX-measurement. The EDX-linescan is marked green. The interface between the amorphous and the crystalline $\mathrm{SiC}$ is marked red.

(bottom) EDX-spectra of the different elements. The length scale was calibrated using the corresponding TEM-image. All spectra were normalized with respect to their particular maximum.

combined with decreasing slope of the spectra, the presumption of an intermixing of carbon and the amorphous $\mathrm{SiC}$ is reasonable.

Regarding the total values of the interface-widths, it has to be noted that the slopes of the different spectra right at the interface blur due to slight thermal drift of 
the TEM-lamella during the measurement. More precisely, the lamella has a drift velocity of approximately $0.5-1 \mathrm{~nm} / \mathrm{min}$ and the duration of each measurement is 5-10 min.

Continuing along the path of the linescan, the Si-spectrum possesses a small valley at around $150 \mathrm{~nm}$ in length of the linescan, matching the area around the exact position of the interface between the amorphous and the crystalline SiC. Therefore, it is marked red in figure 5.27 (top). After that, the Si signal increases slightly and continuously. In contrast, the carbon signal is virtually constant in this area. This is caused by the very low signal to noise ratio (an intensity of 5 counts/s at a noise level of 3 counts/s) of the original dataset. Therefore, even anticipated opposing changes in intensity compared to silicon are beyond the resolution limits of the setup.

As a result, consistency in the stoichiometry of both silicon-carbide phases cannot be verified. A possible, but highly speculative explanation is that the shift in intensity of the Si-signal might originate from a change in density and/or different sputter-yield with respect to a Ga-irradiation during the FIB-preparation.

Nevertheless, the main result of the EDX-analysis is the verification of the amorphous $\mathrm{SiC}$ phase forming the thick amorphous interlayer. Similar the heterostructures based on p-type $\mathrm{Si}$ (see sections 5.3.4 and 5.3.5), the two interface configurations shown in the performed TEM-analyses of $\mathrm{SiC}$ based samples also originate most likely from the original surface properties of the silicon carbide wafers. In this context, the formation of an amorphous layer might be caused by the polishing process of each wafer.

In addition, a closer look at the interface between ta- $\mathrm{C}$ and the a-SiC at high magnifications (e.g. 560k) provides additional evidence for the existence of an intermixing layer originating from ion implantation of pure carbon into the amorphous $\mathrm{SiC}$. Such a layer can be identified based on the change in contrast with respect to the amorphous SiC-layer as well as the ta-C thin-film and is marked by the red dashed line in figure 5.24 .

Assuming an amorphous SiC surface with the common mass density of $3.21 \mathrm{~g} / \mathrm{cm}^{3}$ for the TRIM-calculations, the irradiation with carbon-ions at an energy of $100 \mathrm{eV}$ would lead to the formation of a very thin intermixing layer with a maximum range of about $1.8 \mathrm{~nm}$ based on the ion ranges of $0.8 \mathrm{~nm}$ with a straggling of $0.4 \mathrm{~nm}$ for carbon. In the process, the corresponding sputter-yield has a value 0.252 atoms/Cion favoring silicon atoms with a ratio of 0.175:0.077. The measured thickness of $2.8(3) \mathrm{nm}$, regarding this particular sample, is in accordance with the theoretical value.

Finally, the thickness of the grown ta-C thin-film was measured. The thickness varies between $59.7(3) \mathrm{nm}$ and 70.4(3) nm depending on the position of the measurement with respect to flat sections of the ta-C thin-film. These are separated by bended parts of the heterostructure resulting from surface patterns of the original $\mathrm{SiC}$ which is analyzed and discussed in section 5.3.8 using scanning electronmicroscopy (SEM) images. Nevertheless, the lower threshold of the measurement is in accordance with the calculated value of $65.5 \mathrm{~nm}$ with a difference of about $8.9 \%$. 
In summary, the growth process of turbostratic boron-nitride (t-BN) and tetrahedral amorphous carbon (ta-C) onto a silicon carbide substrate in $6 \mathrm{H}$ configuration can be described in the following way:

During the sputter-cleaning process, the crystalline structure of silicon-substrate is damaged by the $\mathrm{Ar}^{+}$-ions leading to the formation of an amorphous SiCinterlayer with a thickness of about $5 \mathrm{~nm}-7 \mathrm{~nm}$. At the beginning of the deposition process, the t-BN and ta-C each nucleate on top of the flat, but amorphous SiC-surface. The slight deviation between the calculated and the actually measured thickness of the thin-films is mainly caused by the deposition process itself due to a dynamic sputtering processes. 


\subsubsection{Surface properties of $6 \mathrm{H}-\mathrm{SiC}$}

In order to investigate the surface morphology of the silicon carbide substrates utilized in this thesis, scanning electron-microscopy (SEM) images were taken from the surface of virgin $6 \mathrm{H}-\mathrm{SiC}$ substrates as well as from the heterostructures formed by the $\mathrm{t}-\mathrm{BN} / \mathrm{SiC}$ and ta-C/SiC systems right before the FIB-preparation process. The
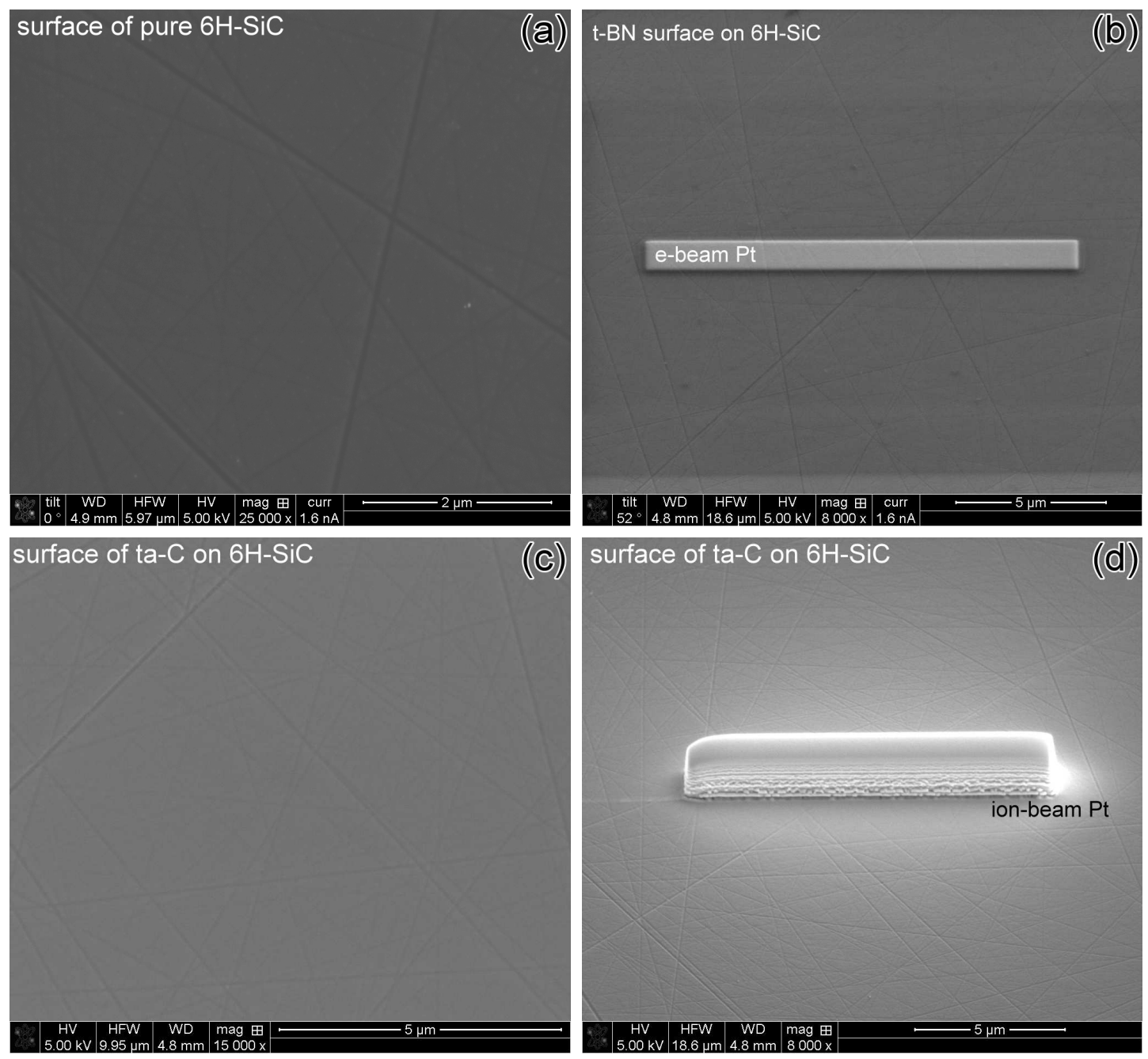

Figure 5.28: SEM-images of different SiC surfaces. The surface of the virgin silicon carbide (a) is traversed by a great number of lines of contrast deviations forming patterns with a triangular and trapezoidal shape. These patters are present at any magnification and even after a complete deposition cycle of t-BN (b) or ta-C (c) including the usual sputter-cleaning process. Furthermore, each single pattern is formed by smaller ones which become visible at higher magnifications. In addition, the different Pt-deposits of the FIB-preparation process are shown in (b) for e-beam Pt and in (d) for ion-beam Pt.

corresponding images are shown in figure 5.28 ,

The surface of the virgin silicon carbide surface (figure 5.28a) is traversed by a great number of lines of contrast deviations forming patterns with triangular and 
trapezoidal shape. These patters are present at any magnification and even after a complete deposition cycle of t-BN (figure 5.28b) or ta-C (figure 5.28c) including the usual sputter-cleaning process. Furthermore, each single pattern is formed by smaller ones which become visible at higher magnifications. These facts indicate either grain boundaries or a deformation of the SiC-surface along the visible straight lines. Regarding the HRTEM-images of the SiC-based heterostructures, there are no evidence for the presence of any grain boundaries inside the $\mathrm{SiC}$ as all images illustrate a single-crystalline structure. Therefore, a deformation of the surface is more likely. A closer look at the cross-section TEM-measurement of a typical heterostructure of the ta-C/SiC system at low magnification confirms this statement. An appropriate image is shown in figure 5.29. The triangular patters in

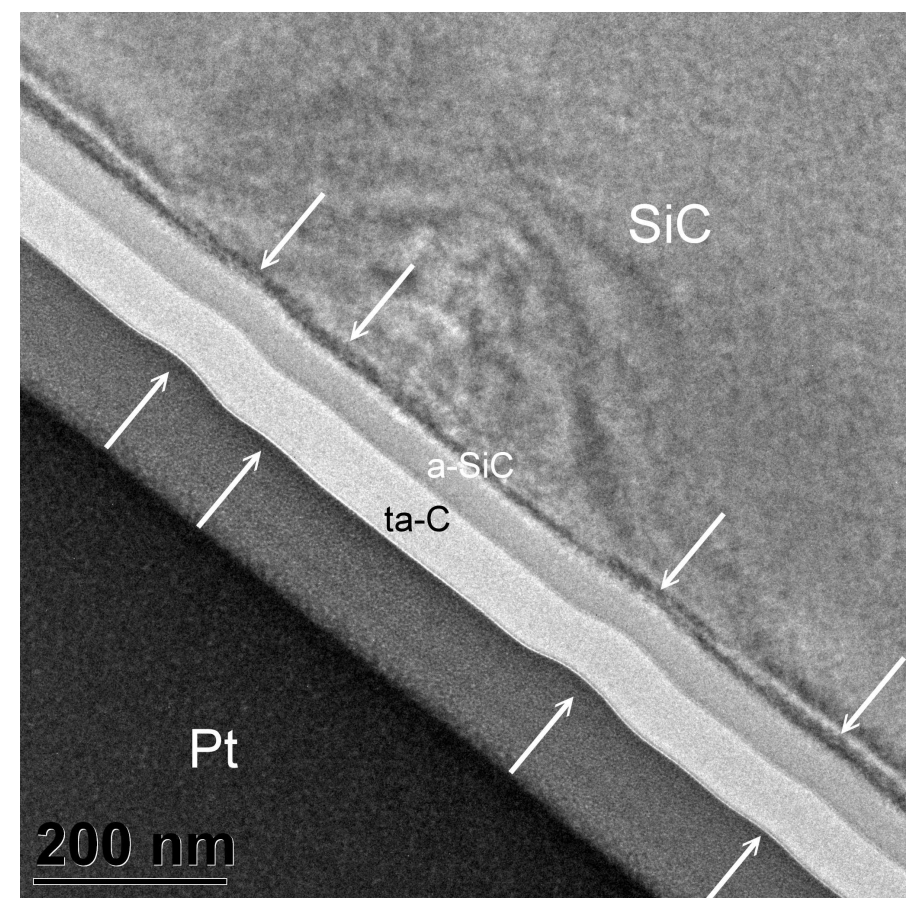

Figure 5.29: Low magnification cross-section TEM-measurement of a typical heterostructure of the ta-C/SiC system. The triangular patters in the SEM-images of figure 5.28 are actually grooves and scratches in the silicon carbide surface, the amorphous as well as the crystalline, occurring at irregular intervals between approximately $150 \mathrm{~nm}$ and $400 \mathrm{~nm}$ and at different depths of about 2-10 nm.

the SEM-images are actually grooves and scratches in the silicon carbide surface, the amorphous as well as the crystalline, occurring at irregular intervals between approximately $150 \mathrm{~nm}$ and $400 \mathrm{~nm}$ and at different depths of about 2-10 $\mathrm{nm}$. As even the virgin $\mathrm{SiC}$ as well as every $\mathrm{SiC}$-based sample exhibit the same patterns regardless of surface treatment, coating material or resolution of the images, an unintentional creation during the sample-preparation-process of this thesis can be ruled out. They likely originate from the from the surface polishing process of the wafers. Unfortunately, additional information from the manufacturer could not be obtained. 
Comparing the results of this work with independent reports in literature of similar patters on the $\mathrm{SiC}$ surface of wafers by different groups, there is proof that these patterns are in fact scratches on the $\mathrm{SiC}$ surface caused by the mechanical polishing process of the $\mathrm{SiC}$ wafers [147, 148]. 


\subsection{Summary}

The successful growth of tetrahedral amorphous carbon (ta-C) as well as turbostratic boron nitride $(\mathrm{t}-\mathrm{BN}) \sqrt[3]{3}$ thin-films has been verified on the basis of phase composition and crystalline micro-structure analyses using in-situ X-ray photoemission spectroscopy (XPS) measurements and subsequently cross-section transmission-electronmicroscopy (TEM), respectively.

Based on the results of the large number of investigated heterostructures using crosssection TEM-measurements, the growth process of the t-BN and ta-C thin-films can be generalized in the following way:

All grown t-BN as well as ta-C thin-films of this thesis each exhibit almost identical, uniform structural properties. In more detail, both types of thin-films contain only a single type structural configuration of the deposited material. The ta-C thinfilms feature an entirely amorphous structure while t-BN thin-films always exhibit a "semi-crystalline" structure of hexagonal (0002)-planes oriented perpendicular to each substrate surface and accompanied by a high defect density throughout the entire thin-film. Both structures have additionally been verified using Fast-Fourier Transformations (FFTs) of the recorded TEM-images. Moreover, both of them exhibit a low surface roughness as well as a smooth interface to the underlying substrate material.

In addition, $\mathrm{Si}$ and $\mathrm{SiC}$ based heterostructures also exhibit amorphous interlayers between the grown thin-films and the particular substrate surface which is formed during the sputter-cleaning process using $1 \mathrm{keV} \mathrm{Ar}^{+}$-ions prior to the deposition of the thin-films. The interface of the t-BN/ZnO system, on the other hand, does not exhibit such amorphous interlayers. Therefore, the formation of such an amorphous layer can exclusively be linked to damage cascades caused by implanted ions during the sputter-cleaning process based on corresponding TRIM-calculations. In this context, the particular ion ranges and recoil effects are primarily based on the material properties of the single crystalline semiconducting substrates. Despite assuming an amorphous matrix, the TRIM-calculations are in good agreement with the TEM-analyses of the resulting depths of the damaged substrate surface forming the amorphous interlayer.

Moreover, further deviations from the anticipated structural properties in the form of additional thick amorphous layers inside several p-type silicon and silicon carbide based samples have been linked to the original condition of the wafer surfaces prior to the sputter-cleaning process while the consistency regarding the composition of the layers has been verified using EDX-measurements. Based on the similarities of the particular thin-film structures grown on a variety of different substrates, the actual growth process of the thin-films does not depend on the composition or orientation of the substrate. Due to the amorphous or turbostratic character of the grown thin-films, the electrical properties of all grown MASS heterostructure in this thesis

\footnotetext{
${ }^{3}$ Due to the ion based deposition technique, turbostratic boron-nitride is formed instead of the hexagonal phase.
} 
will likely be affected by strong contribution from conduction mechanisms related to amorphous or highly disordered materials like Hopping mechanisms, Frenkel-Poole or Schottky emission (see sections 3.2, 3.3 and 3.4). Furthermore, the amorphous interlayers of p-Si and $6 \mathrm{H}-\mathrm{SiC}$ based MASS systems will also likely result in an additional contribution from a defect related conduction mechanism.

Focusing on the actual growth process, the particular thin-films nucleate on top of the flat substrate surface 4 right after the deposition of a few mono-layers (equal to $\mathrm{x} \cdot 10^{15}$ ions) of atoms. In the process, an intermixing layer is formed in each system due to implantation of deposited ions into the surface layer of the semiconducting material at the beginning of the deposition process. The resulting dimensions of these layers obtained from the HRTEM-measurements are basically consistent with additionally performed TRIM-calculations of the deposition process. However, the exact ion-ranges and, in particular, the resulting compositions of the intermixing layers of the different systems depend on the original material properties of the single crystalline substrates as well as on the collision cascades of the implanted ions and the resulting configuration inside the surface layer of the substrate.

In addition, the mixing process might also lead to an incorporation of corresponding substrate- or dopant-atoms into the grown thin-films. The distribution of such kind of atoms inside the grown thin-films would depend on the solubility inside the particular thin-film matrices as well as on the corresponding sputter-yields and recoil distributions of these atoms. Furthermore, either a gradual distribution or a segregation process towards the surface would be the most likely results due to the dynamic sputtering process during deposition of the thin-films. However, due to the lack of an appropriate sensitive analysis technique regarding the detection of single atoms inside an $2 \mathrm{~nm}$ thick defect-rich intermixing layer, these assumptions are just circumstantial. Nevertheless, the electrical properties of the grown thin-films might be affected by these kind of processes.

Focusing on the lattice constants of the single crystalline substrates, all of them are in very good agreement with the corresponding values derived from the latticeconstants taken from literature. Furthermore, the discrepancies regarding the lattice constants of the (0002)-planes inside the grown t-BN thin-films can be linked to the high density of defects and dislocations inside the turbostratic structure.

Finally, regarding the film-thicknesses obtained from the TEM-images, ta-C as well as t-BN thin-films each exhibit an almost constant loss of thickness compared to the calculated values based on the deposited charge and material parameters according to equation 2.1, more precisely 5\%-10\% for ta-C and $30 \%-35 \%$ for t-BN thin-films. In this context, the loss of thickness regarding each type of thin-film is consistent in all investigated heterostructures regardless of the particular substrate material. The corresponding values of all samples investigated in the scope of this thesis are summarized in table 5.3. Taking into account additional TRIM calculations regarding the sputter processes during the actual growth of the thin-films, the

\footnotetext{
${ }^{4}$ primarily realized by the polishing process right after fabrication of the wafers and by the sputter-cleaning process prior to deposition of the thin-films
} 


\begin{tabular}{|c|c|c|c|c|}
\hline $\begin{array}{c}\text { heterostructure } \\
\text { system }\end{array}$ & sample & $\begin{array}{c}\text { calculated } \\
\text { thickness } \\
{[\mathrm{nm}]}\end{array}$ & $\begin{array}{c}\text { measured } \\
\text { thickness } \\
{[\mathrm{nm}]}\end{array}$ & $\begin{array}{c}\text { difference } \\
{[\%]}\end{array}$ \\
\hline t-BN/n-Si & 1221 & 86.6 & $57.6(19)$ & 34.9 \\
\hline ta-C/n-Si & $\begin{array}{l}1245 \\
1272\end{array}$ & $\begin{array}{l}20.6 \\
41.2\end{array}$ & $\begin{array}{c}20.4(3) \\
38.6\end{array}$ & $\begin{array}{c}- \\
6.3\end{array}$ \\
\hline $\mathrm{t}-\mathrm{BN} / \mathrm{ZnO}$ & $\begin{array}{l}1174 \\
1220\end{array}$ & $\begin{array}{c}183.5 \\
93.6\end{array}$ & $\begin{array}{c}130.2 \\
64.3(5)\end{array}$ & $\begin{array}{c}30 \\
31.3\end{array}$ \\
\hline ta-C/p-Si & $1305 \mathrm{a}$ & 65.2 & $61.2(3)$ & 7.2 \\
\hline $\mathrm{t}-\mathrm{BN} / \mathrm{SiC}$ & $\begin{array}{c}1242 \\
1246 \mathrm{~b} \\
1274 \mathrm{a}\end{array}$ & $\begin{array}{l}65.2 \\
65.2 \\
81.5\end{array}$ & $\begin{array}{c}41.6(4) \\
42.2(2) \\
55.8\end{array}$ & $\begin{array}{l}36.2 \\
35.0 \\
31.5\end{array}$ \\
\hline ta-C/SiC & $\begin{array}{l}1308 \mathrm{a} \\
1320 \mathrm{a}\end{array}$ & $\begin{array}{l}65.5 \\
73.1\end{array}$ & $\begin{array}{l}59.7(3) \\
65.4(3)\end{array}$ & $\begin{array}{c}8.9 \\
10.5\end{array}$ \\
\hline t-BN/p-Si & $\begin{array}{c}1196 b \\
1197\end{array}$ & $\begin{array}{l}97.5 \\
93.4\end{array}$ & $\begin{array}{l}66.9(3) \\
59.1(6)\end{array}$ & $\begin{array}{l}32 \\
36\end{array}$ \\
\hline
\end{tabular}

Table 5.3: Comparison of calculated film thicknesses and measured values obtained from TEMmeasurements.

difference between the calculated and the actually measured thickness of the t-BN and ta-C thin-films is likely caused by dynamic sputtering effects of the deposited ions themselves accompanying the actual growth process. Based on these results, the thin-film thickness of each sample can be calculated with high accuracy in future experiments based on the deposited charge and sample geometry using equation 2.1 and applying a correction factor of each thin-film material. 


\section{Chapter 6}

\section{Electrical characterization}

A significant part of this thesis is the investigation of the electrical properties of different MASS diode heterostructures (see figure 2.1). Direct current (DC) measurements were performed at different temperatures according to the description of section 4.2 and interpreted on the basis of the conduction mechanisms mentioned in chapter 3. In this context, the main issue is the development of a general, temperature dependent conduction model for all MASS diode systems.

In the following, the initial development of such a conduction model will be illustrated step by step. Furthermore, several essential extensions, mainly based on the structural properties of the different heterostructures, will be taken into account and explained in detail. In addition, preliminary investigations of each particular semiconducting substrate were performed regarding different back-contact materials and preparation steps (see section 2.3) prior to the actual electrical characterization of the different MASS diode systems.

In a first step, the current-voltage ( $\mathrm{I}-\mathrm{V})$ characteristics of ta-C and $\mathrm{t}-\mathrm{BN}$ reference samples on highly doped n-type Si-substrates were investigated at different temperatures in order to verify the conduction mechanism of the grown thin-films. In addition, general limits can be set for potential fit parameters of the amorphous semiconductor part in MASS diode heterojunctions.

In a next step, the I- $\mathrm{V}$ characteristics of $\mathrm{t}-\mathrm{BN} / \mathrm{ZnO}$ as well as ta-C/Si MASS diodes were investigated at temperatures of $20 \mathrm{~K}-300 \mathrm{~K}$. In this context, the I-V characteristics are well described by the so called Frenkel-Poole and ideal diode model (FPID-model) based on a serial arrangement of an ideal Schottky diode, a FrenkelPoole type resistor and an ohmic resistor. As a side effect, comparisons with a more simplified non-ideal diode model (NID-model) provide possible explanations of apparently high ideality factors mentioned in literature (see chapter 11). A significant part of these findings, more precisely the development of the FPID-model itself based on selected I-V characteristics measured at room temperature (RT), was already published in the scope of this thesis [53, 149]. In addition, the model is verified by temperature dependent measurements and related numerical fits using python scripts. 
In order to extend the validity of the model, it was applied to less rectifying I-V characteristics of $\mathrm{SiC}$ based MASS diodes. In comparison to previous results, these heterojuctions show an increased leakage current at reverse-bias. Fortunately, these results can be directly linked to the structural properties of the heterostructures (see chapter 5) leading to an extension of the FPID model. This extension of the model based on $\mathrm{I}-\mathrm{V}$ characteristics of $\mathrm{t}-\mathrm{BN} / \mathrm{SiC}$ heterojunctions at RT was also already published during the completion of this thesis [150].

In the end, all results will be merged leading to a successful development of a general and temperature dependent DC conduction model for all MASS diodes.

\subsection{I-V characteristics of reference samples}

According to previous publications by Ronning et al. [57, 60] and Hofsäss et al. 151 as well as to corresponding results published during the scope of this thesis [53, 149, 150], turbostratic boron-nitride (t-BN) and tetrahedral amorphous carbon (ta-C) thin-films both basically exhibit a conduction mechanism similar to FrenkelPoole emission (see section 3.3).

In order to verify Frenkel-Poole like conduction as the dominant mechanism inside the grown amorphous and disordered thin-films, sufficient quantities of reference samples regarding both types of thin-films were fabricated and characterized electrically by performing temperature dependent measurements during this work. They were grown on highly doped n-type silicon substrates (see section 2.2.1). Based on the resistivity of $(0.005-0.01) \Omega \mathrm{cm}$, these substrates are referred to as "metallic" silicon.

The electrical properties of these substrates were investigated using Ag back-contacts as well as evaporated $\mathrm{Au} / \mathrm{Cr}$ contacts on the polished top-side. The corresponding I-V characteristics are exemplarily shown in figure 6.1. In the example at hand, the resulting I-V characteristic is perfectly linear and exhibits a very low resistance of about 1.25(2) $\Omega$, which has been determined using a linear fit (red) indicating no formation of any interfering Schottky barriers between the silicon and the particular metal contact. It has to be noted, that both sides of the substrate were etched prior to the application of $\mathrm{Ag}$ contacts using hydrofluoric acid in order to remove the commonly present $\mathrm{SiO}_{2}$ surface. In an alternative approach, low ohmic backcontacts can also be realized by partially removing the silicon dioxide via scratching the surface with a small diamond.

Either way, any formation of Schottky barriers between the $\mathrm{Si}$ and $\mathrm{Ag}$ or $\mathrm{Cr} / \mathrm{Au}$ is neutralized by the high density of free charge carriers inside the highly doped substrate. Therefore, highly conductive ohmic contacts with a resistivity of about $1 \Omega$ on highly doped n-type silicon can easily be obtained using Ag. As a result, the electrical characterization of the reference samples is independent of any backcontact resistances.

In the following, the I-V characteristics of ta-C as well as t-BN thin-films have been investigated at different temperatures in order to determine their characteristic pa- 


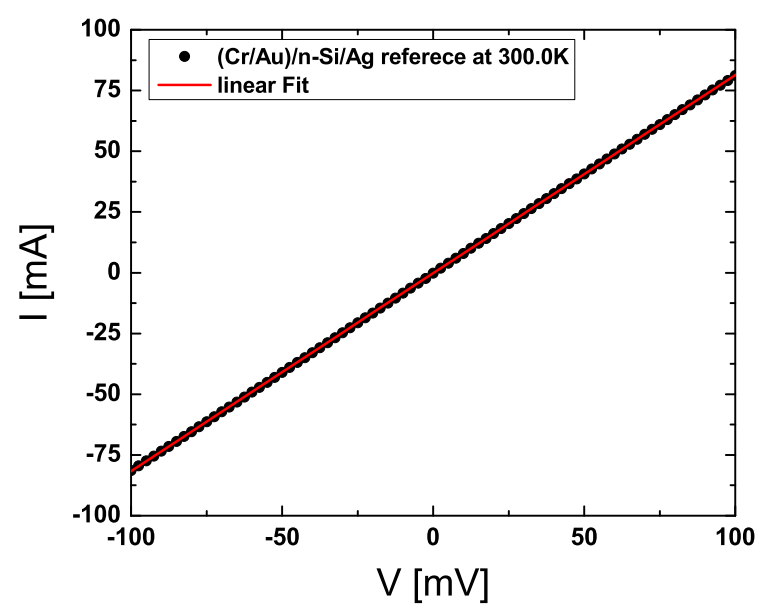

Figure 6.1: I-V characteristic of the $\mathrm{Au} / \mathrm{Cr} / \mathrm{n}-\mathrm{Si} / \mathrm{Ag}$ interface structure. The resistance has been determined to a value of $1.25(2) \Omega$ using a linear fit (red).

rameters according to equation 3.8, especially the average barrier height of the localized traps $\Phi_{\mathrm{FP}}$. In addition, the datasets and determined parameters of this thesis are compared to former reference sample measurements of metal/t-BN/metal structures grown on polished steel substrates and metal/ta-C/metal structures grown on polished nickel substrates, respectively. 


\subsubsection{Metal/t-BN/metallic Si heterostuctures}

Starting with a detailed analysis of t-BN reference samples on metallic n-type silicon substrates, temperature dependent measurements in the range of $20 \mathrm{~K}-383 \mathrm{~K}$ were performed using the low temperature setup (see section 4.2.3) for temperatures up to $300 \mathrm{~K}$ and the high temperature setup (see section 4.2.4) above. The separate I-V datasets were merged and assembled in a single plot for each sample. Corresponding $\mathrm{I}-\mathrm{V}$ characteristics of a typical t-BN reference sample are shown in figure 6.2 for different temperatures in a Schottky-plot $(\log (\mathrm{I})$ versus $\sqrt{\mathrm{V}})$. All I-V curves exhibit a

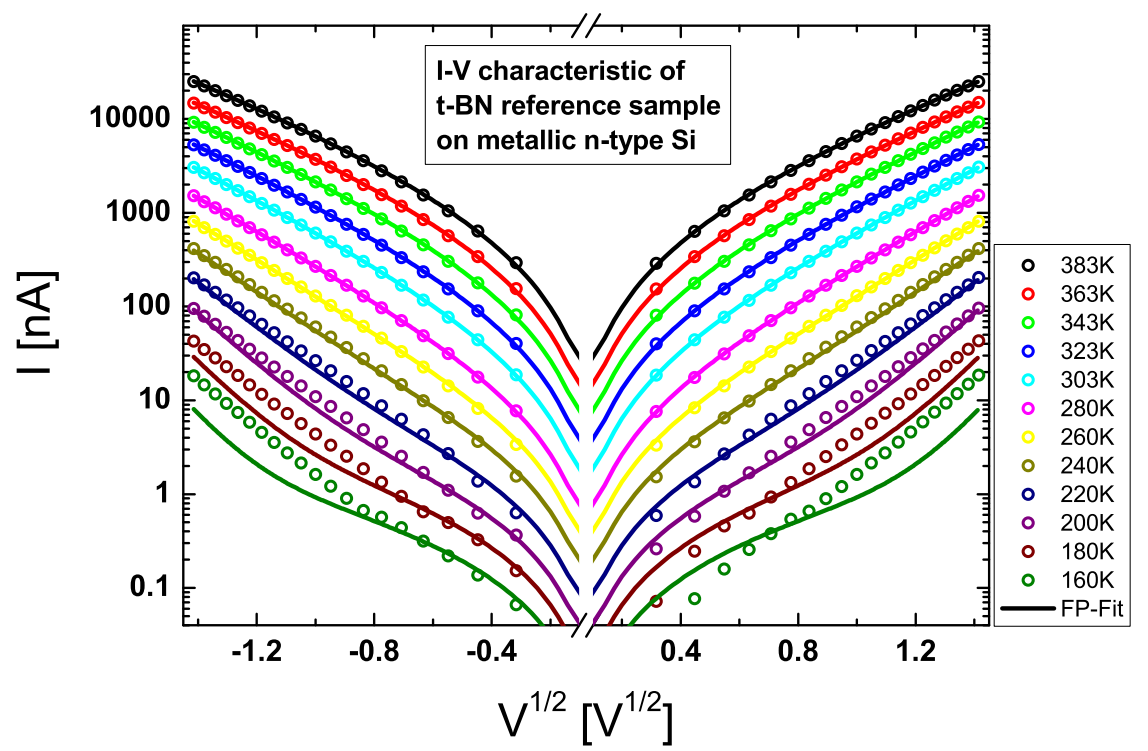

Figure 6.2: Temperature dependent I-V measurements of a typical t-BN reference sample arranged in a Schottky-plot $(\log (\mathrm{I})$ versus $\sqrt{\mathrm{V}})$. All recorded I-V curves (colored circles) exhibit a symmetric behavior and are in good agreement with numerical simulations (colored solid lines) of the FP-model of this work based on equation 6.1 regarding a parallel arrangement of a FrenkelPoole resistor $R_{\mathrm{FP}}$ and a parasitic resistance $R_{\mathrm{P}}$ according to figure 6.3 . down to a temperature of about $240 \mathrm{~K}$. Below that temperature, the fits start to deviate from the measured data indicating the presence of an additional conduction mechanism dominating at lower temperatures.

symmetric behavior and can be well described by a parallel arrangement of a FrenkelPoole resistor $R_{\mathrm{FP}}$, dominating the characteristic at higher temperatures and electric fields, and a parasitic resistance $R_{\mathrm{P}}$ which may also include contributions from variable range hopping and/or tunneling at lower electric fields and temperatures. Therefore, $R_{\mathrm{P}}$ is an ohmic resistor in first-order approximation. An appropriate equivalent circuit diagram of the Frenkel-Poole model in this thesis is illustrated in figure 6.3 and the corresponding current is given by

$$
I=\frac{V}{R_{\mathrm{P}}}+A \cdot \sigma_{0} \frac{V}{d} \exp \left[-\frac{q}{k T}\left(\Phi_{\mathrm{FP}}-\sqrt{\frac{q V}{\pi \epsilon_{0} \epsilon_{r} d}}\right)\right] .
$$




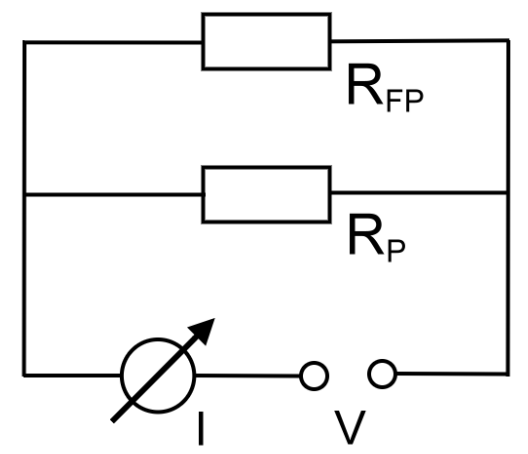

Figure 6.3: Equivalent circuit diagram of the Frenkel-Poole model (FP-model) of this work describing the I-V characteristics of metal/amorphous semiconductor/metallic Si heterostructures based on a parallel arrangement of a Frenkel-Poole like resistor $R_{\mathrm{FP}}$ and a parasitic resistor $R_{\mathrm{P}}$ in accordance to equation 6.1 .

In this context, the parameters of the FP-resistor are identical to those in equation 3.8. The original prediction of possible conduction mechanisms like Frenkel-Poole emission accompanied by hopping or tunneling is primarily based on the structural properties of the grown thin-films obtained from the TEM-analysis in section 5.3.2. In this context, the high density of dislocations and defects inside the grown thinfilms are particularly most accountable for the resulting conduction mechanisms.

In terms of mathematics, the large number of free parameters in equation 6.1 leads to to an over-determined system of equations. As a result, the range of vital parameters has to be narrowed down prior to the fitting process and preferably kept constant with respect to $T$ for a whole array of curves based on physical reasons. In this context, the most important parameter is the average barrier height $\Phi_{\mathrm{FP}}$. It is determined by comparing two adjacent I-V characteristics measured at different temperatures with a small temperature difference $\Delta T / T \leq 0.2$, e.g. $10 \mathrm{~K}$. In the process, the linear region in the Schottky-plots are extrapolated towards $\sqrt{\mathrm{V}} \rightarrow 0$ leading to a value of $I(0)=I(\sqrt{\mathrm{V}} \rightarrow 0)$. Therefore, $\Phi_{\mathrm{FP}}$ can be obtained by

$$
\Phi_{\mathrm{FP}} \approx \frac{T_{1} T_{2}}{T_{1}-T_{2}} \frac{k}{q} \ln \left(\frac{I_{T 1}(0)}{I_{T 2}(0)}\right) .
$$

In order to guarantee reproducibility, this procedure was repeated for several samples at a temperature range of $250 \mathrm{~K}-365 \mathrm{~K}$. In this context, the lower threshold of $250 \mathrm{~K}$ is based on a significantly increased margin of error regarding the linear extrapolation below that temperature resulting from an insufficient number of datapoints forming the linear region of the characteristic in a Schottky-plot. A further increase of field intensity exceeding the typical maximum of about $1 \cdot 10^{6} \mathrm{~V} / \mathrm{cm}^{2}$ is not a viable option as this frequently led to an abrupt and irreversible increase of conductivity in the particular probed sample regions. Nevertheless, the average barrier height, determined in this thesis, has an average value of $\Phi_{\mathrm{FP}, \mathrm{t}-\mathrm{BN}}=0.33(2) \mathrm{V}$ and is in very good agreement with previously published values of $\Phi_{\mathrm{FP}}$ on metal and metallic silicon substrates, in detail $\Phi_{\mathrm{FP}, \mathrm{t}-\mathrm{BN}}=0.34(2) \mathrm{V}$ (t-BN on polished steel substrates) and $\Phi_{\mathrm{FP}, \mathrm{t}-\mathrm{BN}}=0.32(2) \mathrm{V}$ (t-BN on metallic n-type Si substrates) [53, 150]. For a known average barrier height $\Phi_{\mathrm{FP}}$ and a film thickness $d$, the relative static permittivity $\epsilon_{r}$ and the conductivity $\sigma_{0}$ can be obtained from numerical fits, 
displayed as solid lines in figure 6.2. Both of them exhibit temperature dependencies according to an $\exp (1 / \mathrm{T})$ relation. In order to limit the range of possible values prior to the fitting-process, the conductivity of the thin-film $\sigma_{0}$ can alternatively be estimated using the slope of the I-V characteristics close to zero-bias:

$$
\sigma_{0} \approx\left(\left.\frac{d I}{d V}\right|_{V \rightarrow 0}-\frac{1}{R_{P}}\right) d \exp \left(q \Phi_{\mathrm{FP}} / k T\right)
$$

In addition, the relative static permittivity $\epsilon_{r}$ with respect to equation 6.1 can also be estimated from the inverse slope of the Schottky plot corrected by a term of $2 / \sqrt{\mathrm{V}}$ due to the linear contribution in the I-V curve:

$$
\epsilon_{r} \approx \frac{q^{3}}{k^{2} T^{2} \pi \epsilon_{0} d}\left[\frac{d\left(\ln \left(I / I_{0}\right)\right.}{d(\sqrt{V})}-\frac{2}{\sqrt{V}}\right]^{-2} .
$$

Comparing the resulting numerical fits to the measured datasets of different temperatures, they are in good agreement down to a temperature of about $240 \mathrm{~K}$. Below that temperature, in a transition region down to $200 \mathrm{~K}-220 \mathrm{~K}$, the fits start to deviate from the measured data indicating the presence of an additional conduction mechanism dominating at lower temperatures. Furthermore, the measured datasets become temperature independent with decreasing temperature, especially below approximately $125 \mathrm{~K}$. $\Phi_{\mathrm{FP}}$ has been kept constant for all numerical fits based on theory that such a barrier height is constant or even increases at lower temperatures. The corresponding essential parameters of the fits according to equation 6.1 regarding each investigated sample of this work are compared and summarized in table 6.1 for room temperature (RT) measurements, also including a partially $\mathrm{Cu}$-contaminated sample (\#1286). In comparison to common t-BN reference samples the conductivity is increased by a factor of around 20 and exhibits a more pronounced curvature of the characteristic. As a result, the values of the parameters $\epsilon_{r}$ and $\sigma_{0}$ change significantly. However, the average barrier height based on the analysis performed

\begin{tabular}{|c||c|c|c|c|c|c|c|}
\hline sample & substrate & $\begin{array}{c}d \\
{[\mathrm{~nm}]}\end{array}$ & $\begin{array}{c}\text { cont. diam. } \\
{[\mathrm{mm}]}\end{array}$ & $\begin{array}{c}\Phi_{\mathrm{FP}} \\
{[\mathrm{V}]}\end{array}$ & $\begin{array}{c}\sigma_{0} \\
{[\mathrm{mS} / \mathrm{m}]}\end{array}$ & $\zeta \cdot \epsilon_{r}$ & $\begin{array}{c}R_{\mathrm{P}} \\
{[\mathrm{M} \Omega]}\end{array}$ \\
\hline \hline $1185 \mathrm{~b}$ & $\mathrm{n}^{++}-\mathrm{Si}$ & 93 & 1.08 & $0.32(2)$ & 0.82 & 19.0 & 9.78 \\
\hline 1221 & $\mathrm{n}^{++}-\mathrm{Si}$ & 65 & 1.08 & $0.32(6)$ & 0.33 & 20.03 & 11.6 \\
\hline $1286 \mathrm{a}$ & $\mathrm{n}^{++}-\mathrm{Si}$ & 80 & 0.9 & $0.35(4)$ & 1.76 & 24.27 & 7.51 \\
\hline $1286 \mathrm{~b}^{* *}$ & $\mathrm{n}^{++}-\mathrm{Si}$ & 80 & 0.9 & $0.31(5)$ & 29.47 & 18.37 & 0.13 \\
\hline $1322 \mathrm{a}$ & $\mathrm{n}^{++}-\mathrm{Si}$ & 63 & 0.9 & $0.33(2)$ & 1.14 & 20.22 & 12.2 \\
\hline
\end{tabular}

Table 6.1: Frenkel-Poole parameter comparison of t-BN reference samples. Notes: $* * \mathrm{Cu}-$ contaminated area.

according to equation 6.2 is almost identical common samples and therefore unaffected by the small amounts of $\mathrm{Cu}$ contaminants inside the thin-film. As a result, these kind of samples can also conclusively be described by the Frenkel-Poole model of this thesis. The effect of $\mathrm{Cu}$-contaminants on the electrical properties of grown 
thin-films of this thesis is analyzed in more detail in section 6.1.3.

In addition, a significant observation regarding table 6.1] is the consistent rather high value of $\epsilon_{r}$ in the range of 15-25 compared to typical values of about 5 regarding such kind of t-BN thin-films, e.g. performed and reported by Fukarek et al. using in-situ ellipsometry measurements during thin-film growth [152].

Based on the simplifications of the model, described in section 3.3, the FP-model cannot be improved or extended with respect to a more realistic dielectric constant. Even at high temperatures above RT, the change in curvature of the characteristic cannot be simulated by changing the remaining parameters. In order to guarantee consistency, the parameter $\epsilon_{r}$ is only bound to the shape of the particular I-V characteristics throughout this thesis. Alternatively, $\epsilon_{r}$ in equation 6.1 can be replaced by $\zeta \cdot \epsilon_{r}$, where $\epsilon_{r}$ is the real dielectric constant of the thin-film and $\zeta$ a factor related to the curvature of the $\mathrm{I}-\mathrm{V}$ characteristic

$$
I=\frac{V}{R_{\mathrm{P}}}+A \cdot \sigma_{0} \frac{V}{d} \exp \left[-\frac{q}{k T}\left(\Phi_{\mathrm{FP}}-\sqrt{\frac{q V}{\pi \epsilon_{0} \zeta \epsilon_{r} d}}\right)\right] .
$$

In a further step, $\mathrm{I}(\mathrm{T})$ analyses were performed based on the measured datasets. The current is plotted on a logarithmic scale as a function of temperature in an Arrhenius-plot for multiple constant bias voltages and displayed for a typical t-BN reference sample in figure 6.4. For clarity reasons, especially regarding the overlap of axis captions and data-points at higher temperatures in an Arrhenius-plot, the datasets of the LT- and HT-setup were split into separate graphs. For high temperatures between $300 \mathrm{~K}$ and $365 \mathrm{~K}$ almost perfect linear characteristics can be observed indicating an $\exp (1 / \mathrm{T})$-relation of the current and the corresponding conductivity $\sigma_{0}$ as expected for conduction mechanism dominated by thermal excitation like FrenkelPoole conduction (see section 3.3). At lower temperatures, the I(T)-characteristic starts to bend slightly with decreasing temperature. However, the determined average barrier height is almost constant down to the threshold temperature of $250 \mathrm{~K}$ as described above. Furthermore, the $\mathrm{I}(\mathrm{T})$ characteristic also exhibits a transition region between $250 \mathrm{~K}$ and $220 \mathrm{~K}$ featured by increased bending. The process is also accompanied by a change in bending of the I-V characteristic and represented by a change in the corresponding parameter $\zeta \cdot \epsilon_{r}$. Below a characteristic temperature of about $200 \mathrm{~K}-220 \mathrm{~K}$, the dominating conduction mechanism changes drastically. This can be demonstrated by comparing the I(T) characteristics to simulated datasets, marked by the dashed green line in figure 6.4 and $\mathrm{b}$. The simulated data has been calculated for a mid-level constant bias voltage of $1.2 \mathrm{~V}$ based on equation 6.1 using the parameters obtained from the numerical fits in figure 6.2 in the temperature range of $300 \mathrm{~K}-150 \mathrm{~K}$. The I-V characteristics become increasingly independent of temperature leading to the formation of a "plateau" region below $125 \mathrm{~K}$ in the Arrhenius-plot. At the same time, the symmetry of the particular I-V characteristics as well as a Frenkel-Poole emission like behavior are still intact. Therefore, this kind of residual conductivity regarding the $\mathrm{I}(\mathrm{T})$ characteristic of particular bias voltages is similar to the tunneling mechanism of the systems described in section 3.7 . 

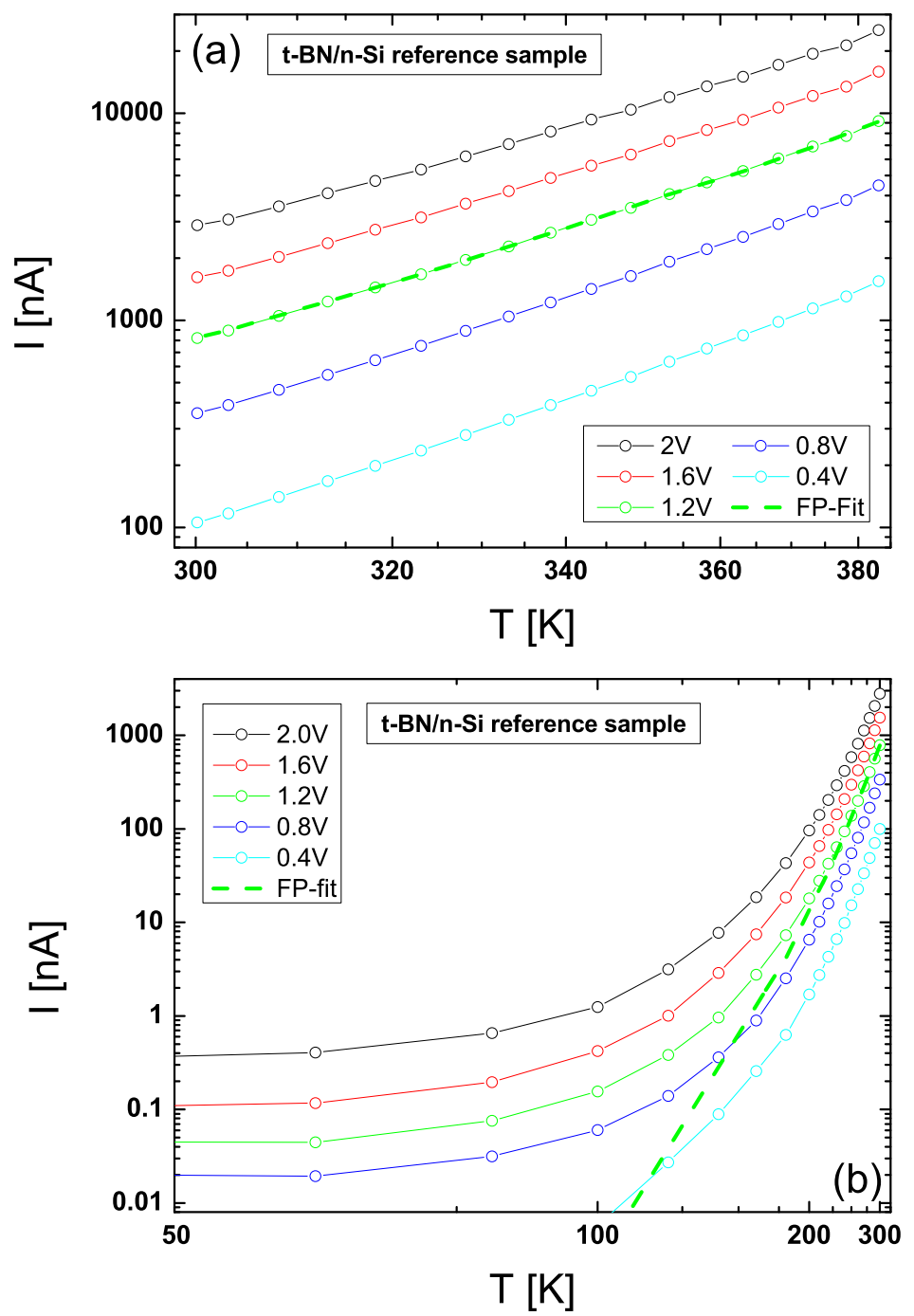

Figure 6.4: Arrhenius-plots of a typical t-BN reference sample for multiple constant bias voltages. (a) High temperature setup: between $300 \mathrm{~K}$ and $365 \mathrm{~K}$ almost perfect linear characteristics can be observed indicating an $\exp (1 / \mathrm{T})$-relation of the current as expected for FP-conduction. (b) Low temperature setup: the $\mathrm{I}(\mathrm{T})$-characteristic starts to bend slightly with decreasing temperature due to the temperature dependence of the corresponding parameters. At $200 \mathrm{~K}-220 \mathrm{~K}$, the characteristics deviate from the predicted behavior illustrated by simulated datasets (dashed green lines in $\mathrm{a}$ and $\mathrm{b}$ ) for a mid-level constant bias voltage of $1.2 \mathrm{~V}$ based on equation 6.1 and parameters obtained from the numerical fits in figure 6.2 for $\mathrm{T}=300 \mathrm{~K}-150 \mathrm{~K}$. Below $125 \mathrm{~K}$, the I-V characteristics become increasingly temperature independent leading to the formation of a "plateau" region with residual conductivity of the samples. 


\subsubsection{Metal/ta-C/metallic Si heterostuctures}

The I-V characteristics of a typical ta-C reference sample are illustrated in another Schottky-plot for different temperatures in figure 6.5.

In general, the conductivity of ta-C thin-films is significantly higher, e.g. by a factor of about 100 at room temperature, when compared to a t-BN thin-film exhibiting similar dimensions of thickness and contact-area. In a next step, the electrical properties of ta-C reference samples on metallic n-type silicon substrates were investigated using temperature dependent measurements in the range of $20 \mathrm{~K}-383 \mathrm{~K}$. Prior to the actual fitting process, the average activation barrier height $\Phi_{\mathrm{FP}}$ of ta-

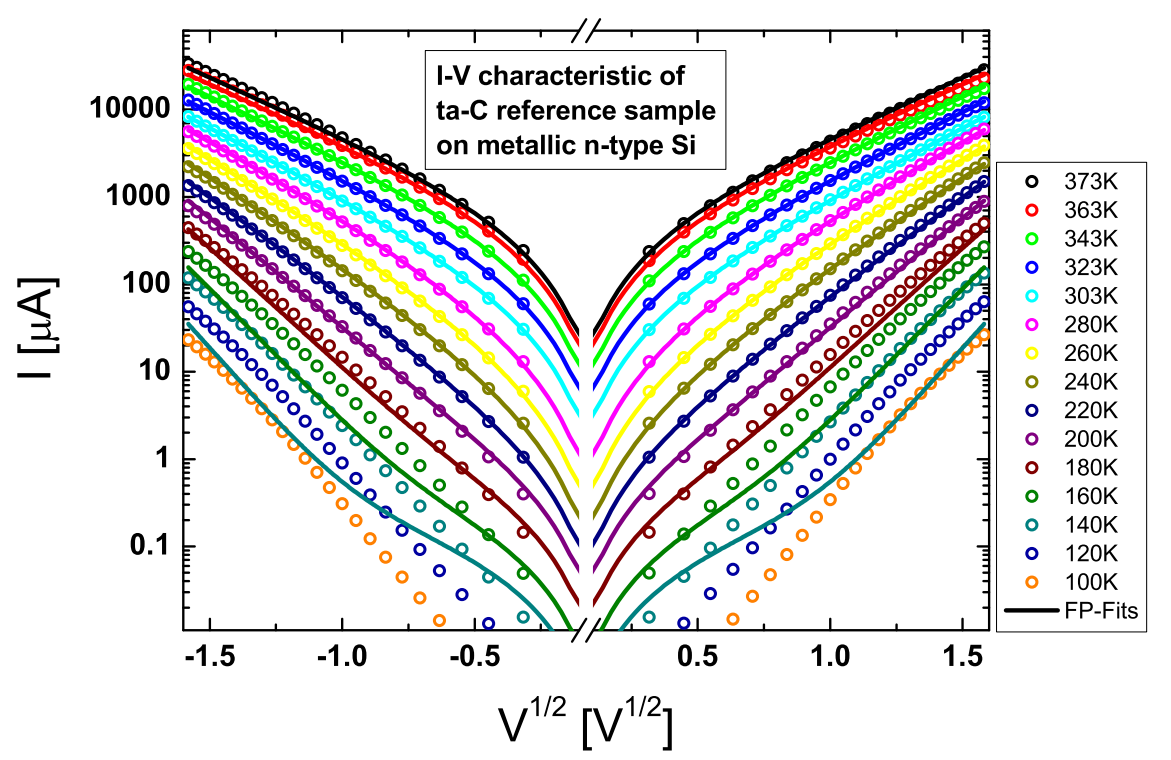

Figure 6.5: Temperature dependent Schottky-plot of a typical ta-C reference sample. All I-V characteristics exhibit a symmetric behavior similar to t-BN and can also be well described by the FP-model in figure 6.3 based on equation 6.1 down to a temperature of about $200 \mathrm{~K}$. At lower temperatures, the numerical fits start to deviate from the original data indicating the presence of an additional conduction mechanism dominating at lower temperatures similar to t-BN samples.

$\mathrm{C}$ was again determined by comparing a linear extrapolation $I(0)=I(\sqrt{V} \rightarrow 0)$ according to equation 6.2 of two I-V curves at adjacent temperatures down to approximately $220 \mathrm{~K}$ leading to an average value of $0.248(6) \mathrm{V}$ based on the samples fabricated during this thesis also including several $\mathrm{Cu}$ contaminated samples.

Despite the change of substrate, this value is in very good agreement with previous results $\Phi_{\mathrm{FP}, \mathrm{ta}-\mathrm{C}}=0.245(5) \mathrm{V}$ of ta-C reference samples grown on polished nickel substrates [149]. In comparison to t-BN thin-films, the lower barrier height might be referable to the high level of homogeneity inside the amorphous structure of ta-C compared to deep level traps at dislocations inside the more crystalline turbostratic structure of t-BN according to the TEM-analyses of both systems in sections 5.3 .2 and 5.3.3. In addition, the leakage current, dominating at low electric fields, is also 
significantly higher in ta-C compared to t-BN thin-films supporting the theory.

Focusing on the recorded datasets in figure 6.5, all I-V characteristics exhibit a symmetric behavior similar to t-BN and can also be well described by the parallel arrangement of a Frenkel-Poole resistor $R_{\mathrm{FP}}$ and an ohmic parasitic resistor $R_{\mathrm{P}}$ illustrated in figure 6.3 according to equation 6.1. The corresponding fits are marked by the colored solid lines in figure 6.5. For a known film thickness $d$, the corresponding parameters $\zeta \cdot \epsilon_{r}$ and $\sigma_{0}$ can be obtained from these numerical fits. Prior to the actual fit, the ranges of both parameters have been narrowed down similar to the t-BN system using equations 6.3 and 6.4. The particular parameters of the fits according to equation 6.1 regarding each investigated sample of this work are compared and summarized in table6.2. Again, the average barrier height of ta- $\mathrm{C}$ is also unaffected

\begin{tabular}{|c||c|c|c|c|c|c|c|}
\hline sample & substrate & $\begin{array}{c}d \\
{[\mathrm{~nm}]}\end{array}$ & $\begin{array}{c}\text { cont. diam. } \\
{[\mathrm{mm}]}\end{array}$ & $\begin{array}{c}\Phi_{\mathrm{FP}} \\
{[\mathrm{V}]}\end{array}$ & $\begin{array}{c}\sigma_{0} \\
{[\mathrm{mS} / \mathrm{m}]}\end{array}$ & $\zeta \cdot \epsilon_{r}$ & $\begin{array}{c}R_{\mathrm{P}} \\
{[\mathrm{k} \Omega]}\end{array}$ \\
\hline \hline $1213 \mathrm{c}$ & $\mathrm{n}^{++}-\mathrm{Si}$ & 82.5 & 0.7 & $0.244(5)$ & 13.65 & 11.78 & 15.37 \\
\hline $1260^{* *}$ & $\mathrm{n}^{++}-\mathrm{Si}$ & 82.5 & 0.7 & $0.247(11)$ & 1637.90 & 30.54 & 0.75 \\
\hline $1287^{* *}$ & $\mathrm{n}^{++}-\mathrm{Si}$ & 62 & 0.9 & $0.258(19)$ & 649.60 & 23.93 & 7.05 \\
\hline $1314 \mathrm{a}$ & $\mathrm{n}^{++}-\mathrm{Si}$ & 73 & 0.6 & $0.242(3)$ & 41.20 & 12.97 & 12.74 \\
\hline $1314 \mathrm{~b}$ & $\mathrm{n}^{++}-\mathrm{Si}$ & 73 & 0.9 & $0.249(9)$ & 106.39 & 19.60 & 10.30 \\
\hline
\end{tabular}

Table 6.2: Frenkel-Poole parameter comparison of ta-C reference samples. ${ }^{* *} \mathrm{Cu}$-contaminants.

by small amounts of $\mathrm{Cu}$ contaminants sample (analyzed as well in more detail in section 6.1.3). Furthermore, the corresponding dielectric constants resulting from the numerical fits of $\mathrm{Cu}$-free samples in the range of 10-20 are too high compared to a value of 4-6 obtained from ellipsometry measurements for diamond-like carbon films by several groups in literature [56, 153, 154]. However, none of them provided sufficient information about possible interference of the substrate surface or a possible interface layer as shown in section 4.1.2. Therefore, the results of these kind of experiments could not be verified or reproduced during this work and comparisons are highly speculative. In addition, several values were also obtained in a way similar to equation 6.4, which is only a very raw estimate as it is eventually based on the original Frenkel-Poole formula and corresponding simplifications. Moreover, most of the characteristics exhibit hysteresis effects and were neither completely symmetric nor linear in the related Schottky plots of those publications. Based on the high sensitivity of the obtained values with respect to small variations of the estimated slope in a Schottky plot, such an analysis is therefore questionable with respect to the determination of precise values of $\zeta \cdot \epsilon_{r}$ from these kind of electrical measurements. At best, the order of magnitude might be obtainable by this method as it was performed in this thesis.

Focusing on the numerical fits in figure 6.5, they are in good agreement with the recorded datasets down to a temperature of about $200 \mathrm{~K}$ followed by a transition region to around $140 \mathrm{~K}-180 \mathrm{~K}$, depending on the particular sample (presence of contaminants, homogeneity, position of the measurement). This region is again characterized by a progressive deviation of the numerical fits from the original data 
indicating the presence of an additional conduction mechanism dominating at lower temperatures similar to t-BN samples. Below that temperature, the I-V characteristics become temperature independent with decreasing temperature. In the whole fitting process, the average barrier height was again kept constant while the parameters $\sigma_{0}$ and $\zeta \cdot \epsilon_{r}$ exhibit a significant temperature dependence similar to t-BN reference samples.

Furthermore, the recorded datasets were also arranged in an Arrhenius-plot in order to investigate the $\mathrm{I}(\mathrm{T})$ characteristic of the ta-C reference samples. For high temperatures of $300 \mathrm{~K}-383 \mathrm{~K}$ the characteristics are linear indicating again an $\exp (1 / \mathrm{T})$ relation related to a conduction mechanism dominated by thermal excitation like Frenkel-Poole conduction similar to t-BN samples. At lower temperatures, the I(T)characteristic starts to bend slightly with decreasing temperature. However, the effect is less pronounced compared to t-BN and the threshold temperature of $220 \mathrm{~K}$ for the determination of the average barrier height is slightly lower. In addition, the $\mathrm{I}(\mathrm{T})$ characteristic of ta- $\mathrm{C}$ samples also exhibits a transition region in the range of $220 \mathrm{~K}$ and $180 \mathrm{~K}$ featured by a progressive bending of the characteristics at various bias voltages. Similar to t-BN, the process is accompanied by a change in shape of the I-V characteristic leading to changes in the corresponding parameters $\zeta \cdot \epsilon_{r}$ and $\sigma_{0}$ of the numerical fits. Below a characteristic temperature of about $140 \mathrm{~K}-180 \mathrm{~K}$, the dominating conduction mechanism changes drastically 1 .

This change is demonstrated by comparing the $\mathrm{I}(\mathrm{T})$ characteristics to simulated datasets, marked by the dashed green line in figure 6.6. The simulated data has been calculated for a mid-level constant bias voltage of $1.5 \mathrm{~V}$ based on equation 6.1 using the parameters obtained from the numerical fits in figure 6.5 in the temperature range of $300 \mathrm{~K}-100 \mathrm{~K}$. The $\mathrm{I}-\mathrm{V}$ characteristics become increasingly independent of temperature leading to the formation of a "plateau" region below $100 \mathrm{~K}-140 \mathrm{~K}$ in the Arrhenius-plot. At the same time, the symmetry of the particular I-V characteristics as well as a Frenkel-Poole emission like behavior are also still intact. Furthermore, the effect of residual conductivity regarding the $\mathrm{I}(\mathrm{T})$ characteristic of particular bias voltages is less distinct than for t-BN reference samples.

\footnotetext{
${ }^{1} \mathrm{~A}$ detailed analysis of ta- $\mathrm{C}$ reference samples in this temperature region is provided by H.-G. Gehrke in reference [155].
} 

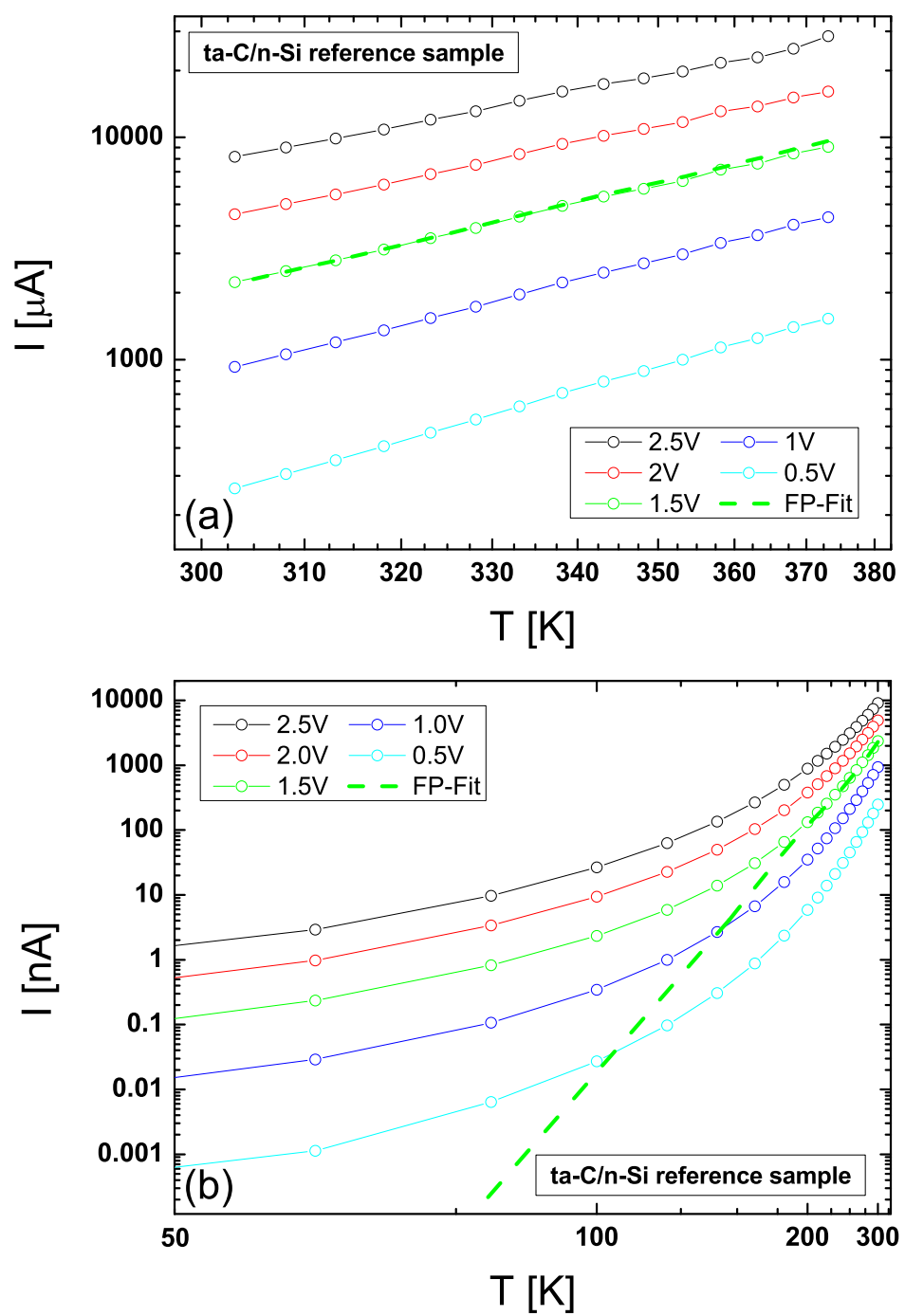

Figure 6.6: Arrhenius-plots of a typical ta-C reference sample. (a) High temperature setup: between $300 \mathrm{~K}$ and $383 \mathrm{~K}$ an almost perfect linear characteristic can be observed indicating an $\exp (1 / \mathrm{T})$-relation of the current as expected for FP-conduction similar to t-BN samples. (b) Low temperature setup: the $\mathrm{I}(\mathrm{T})$-characteristic starts to bend slightly with decreasing temperature due to T-dependencies of the corresponding parameters. At $140 \mathrm{~K}-180 \mathrm{~K}$, the characteristics deviate from the predicted behavior again illustrated by simulated datasets (dashed green lines in a and b) for a mid-level constant bias voltage of $1.5 \mathrm{~V}$ based on equation 6.1 and parameters obtained from the numerical fits in figure 6.5 for $\mathrm{T}=300 \mathrm{~K}-100 \mathrm{~K}$. The $\mathrm{I}-\mathrm{V}$ characteristics become increasingly temperature independent leading to the formation of a "plateau" region with residual conductivity of the samples below $100 \mathrm{~K}-140 \mathrm{~K}$. 


\subsubsection{Contaminant analysis}

Based on the XPS-analysis in section 5.2.3, the effect of $\mathrm{Cu}$ contaminants on the electrical properties of the grown thin-films was additionally investigated.
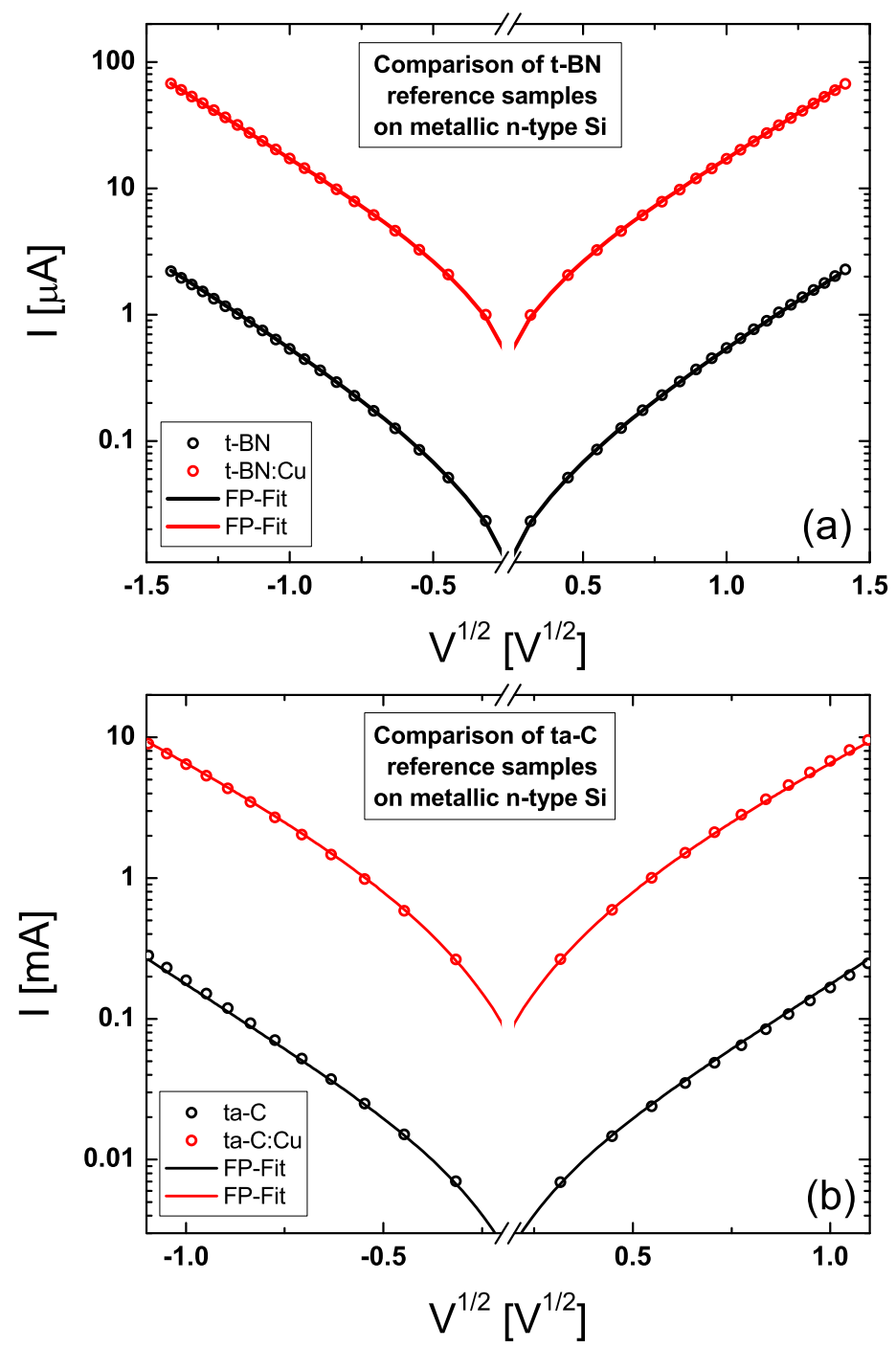

Figure 6.7: Comparison of I-V characteristics (Schottky plots) of typical t-BN (a) and ta-C (b) thin-films on metallic silicon with contaminated samples at room temperature. The additional copper contaminants lead to an increase in conductivity about a factor of approximately 30-40. However, the characteristics still exhibit a symmetric behavior and are qualitatively almost identical to the $\mathrm{Cu}$-free samples. Therefore, the $\mathrm{Cu}$ contaminants inside the t-BN and ta-C matrices eventually lead to an increase in conductivity of the samples but do not basically alter the dominating conduction mechanisms. 
As previously shown in tables 6.1 and 6.2 the corresponding average barrier height is unaffected while the curvature, represented by $\zeta \cdot \epsilon_{r}$, and especially the conductivity of the film $\sigma_{0}$ change. However, the characteristics still exhibit a symmetric behavior and are qualitatively almost identical to the $\mathrm{Cu}$-free samples.

The corresponding I-V characteristics of the LT-setup as well as appropriate numerical fits according to equation 6.1 are exemplarily shown for t-BN as well as ta-C thin-films on metallic silicon in figure 6.7. In this context, characteristic temperature thresholds such as the transition region as well as the region of residual conductivity are very similar to $\mathrm{Cu}$-free samples. As a result, Frenkel-Poole emission is also the dominant conduction mechanism in $\mathrm{Cu}$ contaminated samples above temperatures of about $160 \mathrm{~K}$ for ta- $\mathrm{C}$ and about $200 \mathrm{~K}$ for t-BN thin-films. In addition, comparing the conductivity of comparable copper contaminated t-BN and ta-C reference sample, the resulting factor is very similar to the one obtained for common reference samples in the sections above. Therefore, the $\mathrm{Cu}$ contaminants inside the ta-C matrix eventually lead to an increase in conductivity of the samples but do not basically alter the dominating conduction mechanisms.

Comparing the $\mathrm{I}-\mathrm{V}$ characteristics of typical t-BN and ta-C thin-films on metallic silicon with those of contaminated samples at room temperature the additional copper contaminants lead to an increase in conductivity about a factor of approximately 30-40 in both thin-film systems. However, there is also a significant problem arising from unintentional doping of copper. The electrical stability at high electric fields decreases leading to higher probabilities of electrical breakthrough (abrupt increase in conductivity by several orders of magnitude).

In principle, tiny metal clusters are likely formed during the growth process. Even if most of them are accumulated at the surface of the thin-film (see section 5.2.3), a significant amount of them will be dispersed throughout the amorphous matrix. As a result, they provide possible pathways for a partial or even complete electrical breakthrough. The latter one instantly leads to an ohmic behavior and a resistance in the single digit Ohm range. The former one, on the other hand, often leads to asymmetric I-V characteristics besides a significant increase in conductivity of several orders of magnitude. However, some of them are still symmetric but differ from the commonly recorded characteristics by a significant change in temperature dependence.

The temperature dependence almost vanishes completely indicating the absence of a thermally driven transport mechanism replaced by a temperature independent tunneling mechanism. This is additionally illustrated in an appropriate $\mathrm{I}(\mathrm{T})$ plot in figure 6.8. 


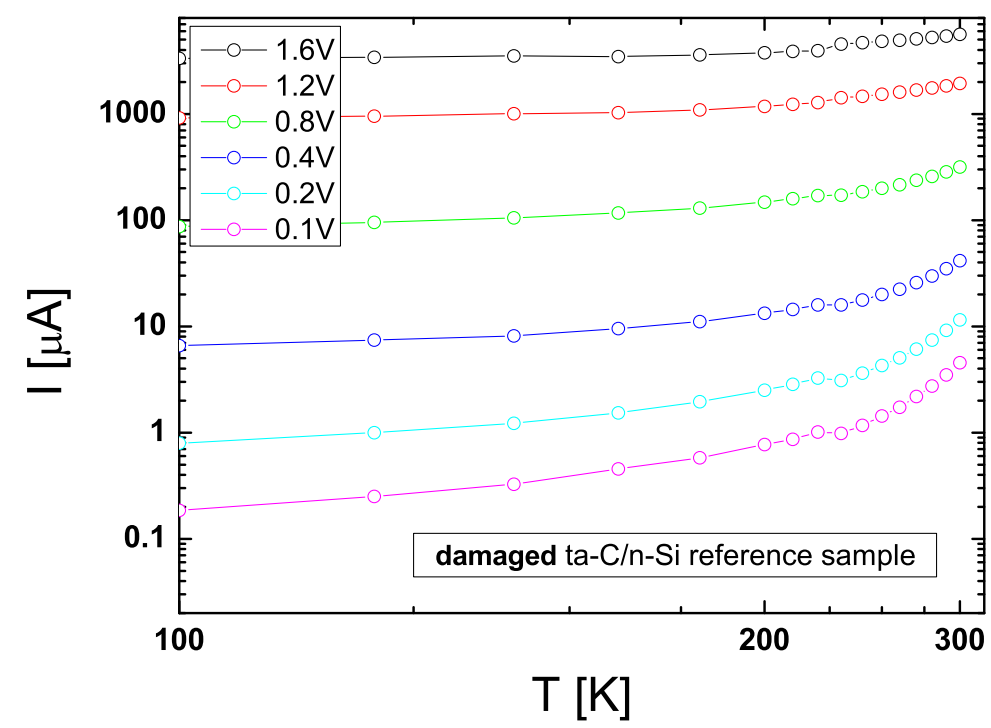

Figure 6.8: Arrhenius-plot of a ta-C reference sample damaged by a partial electrical breakthrough of the thin-film. The temperature dependence almost vanishes completely indicating the absence of a thermally driven transport mechanism replaced by a temperature independent tunneling mechanism. 


\section{$6.2 \quad \mathrm{t}-\mathrm{BN} / \mathrm{ZnO}$ heterostructures}

The first heterojunction investigated in this work are formed by t-BN/ZnO heterostructures with $\mathrm{Cr} / \mathrm{Au}$ contacts evaporated on top. They exhibit a pronounced rectifying characteristic of about three orders of magnitude accompanied by unusual high turn-on voltages of 5-10 V compared to typical p-n junctions (see chapter 1). Furthermore, they possess high stability with respect to electric field intensities across the deposited thin-films in the range of approximately $1 \cdot 10^{7} \mathrm{~V} / \mathrm{cm}$ at a maximum bias voltage of about $70 \mathrm{~V}$. The corresponding I-V characteristic of a typical

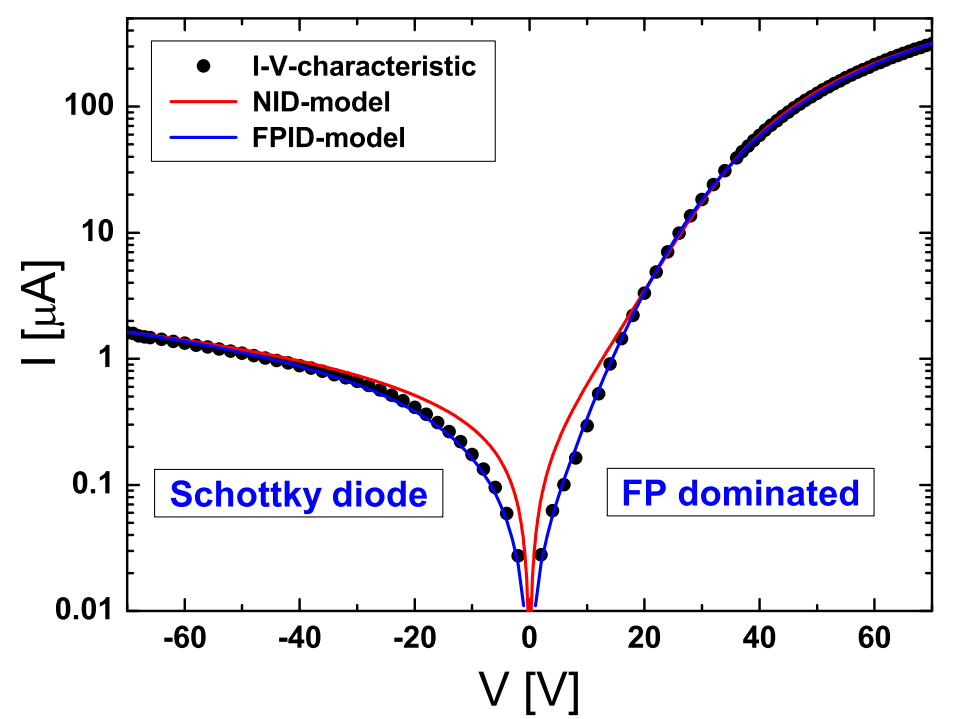

Figure 6.9: $\mathrm{I}-\mathrm{V}$ characteristic of a typical $\mathrm{Au} / \mathrm{Cr} / \mathrm{t}-\mathrm{BN} / \mathrm{ZnO}$ heterojunction at room temperature. The recorded dataset (black circles) can be well described by a serial arrangement of an ideal Schottky diode $\mathrm{D}(n=1)$, a voltage dependent resistor exhibiting Frenkel-Poole like conduction $R_{\mathrm{FP}}$ and an additional ohmic resistor $R_{\mathrm{S}}$ covering present contact and substrate resistances. The numerical simulation (blue solid line) of this Frenkel-Poole and ideal diode model (FPID-model, see figure 6.10) is in very good agreement with the recorded dataset. Furthermore, the MASS diode exhibits an apparently high ideality factor of about 200 according to a non-ideal diode model (NID-model, red solid line) in figure 6.13.

$\mathrm{Au} / \mathrm{Cr} / \mathrm{t}-\mathrm{BN} / \mathrm{ZnO}$ heterostructure is shown in figure 6.9.

The recorded datasets of $\mathrm{Au} / \mathrm{Cr} / \mathrm{t}-\mathrm{BN} / \mathrm{ZnO}$ heterojunctions are well described by a serial arrangement of an ideal Schottky diode $\mathrm{D}(n=1)$, a voltage dependent resistor exhibiting Frenkel-Poole conduction $R_{\mathrm{FP}}$ and an additional ohmic resistor $R_{\mathrm{S}}$ covering present contact and substrate resistances. Furthermore, leakage currents of the Frenkel-Poole resistor and the diode can be approximated by additional parasitic ohmic resistors and are arranged parallel to each one. The resulting equivalent circuit diagram is illustrated in figure 6.10. The corresponding numerical simulation is also illustrated in figure 6.9 by the blue solid line and is in very good agreement 


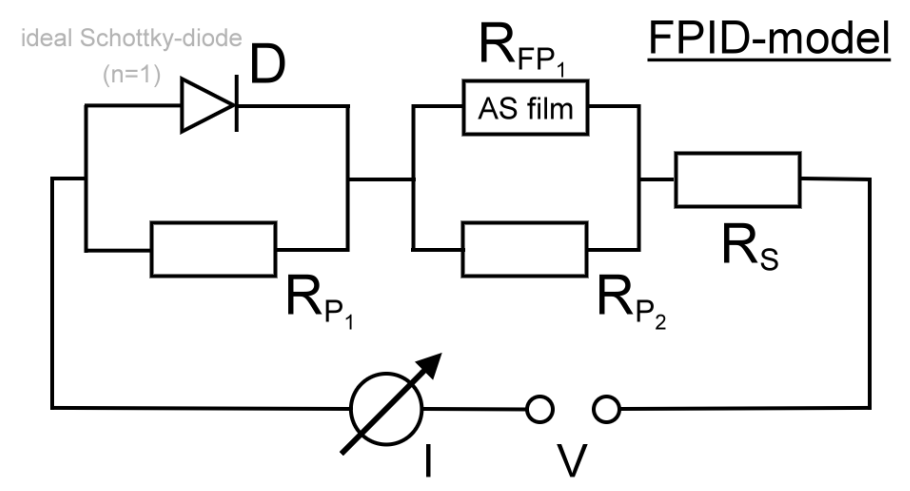

Figure 6.10: Equivalent circuit diagram of the Frenkel-Poole and ideal diode model (FPIDmodel): The MASS diodes are described by a serial arrangement of an ideal Schottky diode $(n=1)$, a voltage dependent resistor $R_{\mathrm{FP}}$ exhibiting Frenkel-Poole conduction and an additional ohmic resistor $R_{\mathrm{S}}$ covering present contact and substrate resistances. The leakage currents of the diode and the Frenkel-Poole resistor can be approximated by additional parasitic ohmic resistors $R_{\mathrm{P} 1}$ and $R_{\mathrm{P} 2}$.

with the recorded data.

In this context, the development of this so called Frenkel-Poole and ideal diode model (FPID-model) is primarily based on the correlation between the structural properties of essential parts with respect to the MASS diode heterostructure and the corresponding electrical properties resulting in the formation of a heterojunction.

First of all, in the absence of any intrinsic doping mechanisms inside the grown thinfilms, the formation of a p-n heterojunction between the "semiconducting" materials of the MASS structure is very unlikely. In addition, based on the results of the t-BN reference samples in section 6.1.1, additional Schottky barriers between the metal top-contacts and the t-BN can also be ruled out.

Therefore, the formation of a depletion region inside the semiconductor caused by the metal top-contact similar to MIS-structures is a reasonable assumption. Hence, the one sided p-n junction is formed between the metal top-contact and the semiconductor substrate and provides the basis for the conduction model. As the structural analysis of t-BN/ZnO heterostructures in section 5.3.1 does not provide any applicable results about the dimensions of such a depletion region and related recombination processes, the diode of this model is presumed to be "ideal" $(n=1) \sqrt{2}$. The related leakage current of the diode at reverse-bias, dominated by the leakage currents inside the depletion region, is described by a high resistive parasitic ohmic resistor $R_{\mathrm{P} 1}$ in first order approximation. Compared to a "virgin" ZnO substrate of the same charge, the resulting leakage current might be increased due to additional free-charge carriers provided by the implanted B- and N-ions at the beginning of the growth process as well as defects caused by the Ar-irradiation during the sputtercleaning process.

\footnotetext{
${ }^{2} \mathrm{~A}$ variation of $\mathrm{n}$ between 1 and 2 does not result in any significant deviations of the FPID-model from the recorded datasets
} 
In contrast, the forward-bias region of this MASS diode is dominated by the grown t-BN thin-film exhibiting Frenkel-Poole like conduction. The electrical properties of the heterojunction itself including interface effects and the amorphous or disordered thin-film have been derived from the structural properties according to the corresponding TEM-analysis in section 5.3.1 for the system at hand.

Based on the perpendicular and direct growth of t-BN on $\mathrm{ZnO}$ substrates accompanied by a very similar structure and orientation of the grown t-BN thin-film itself compared to the t-BN reference samples, the presence of a Frenkel-Poole like resistor according to equation 6.1 dominating the forward-bias direction of the heterojunction is a reasonable assumption. Therefore, the parameters of the corresponding FP-resistor are predominantly based on the results of the t-BN reference samples, especially the average barrier height of $\Phi_{\mathrm{FP}, \mathrm{t}-\mathrm{BN}}=0.33(2) \mathrm{V}$.

Furthermore, another parasitic ohmic resistor $R_{\mathrm{P} 1}$ parallel to $R_{\mathrm{FP}}$ represents possible leakage currents inside the t-BN thin-film. However, for $\mathrm{ZnO}$ based heterostuctures the leakage current of the Frenkel-Poole is several orders of magnitude lower compared to t-BN reference samples and therefore negligible for this system.

Finally, back-contact resistances as well as the intrinsic resistance of the semiconducting substrate can be represented by an additional resistor $R_{\mathrm{S}}$ arranged in series to the essential parts of the MASS heterojunction. Both of these effects were investigated prior to the analysis of the actual heterostructure.

Some of the samples were equipped with $\mathrm{Ti} / \mathrm{Au}$ contacts. They were evaporated based on the procedure described in section 2.3.3. As shown in figure 6.11, the as deposited $\mathrm{Ti} / \mathrm{Au}$ contacts exhibit a linear characteristic indicating ohmic conduction. The corresponding resistance varies from $25 \mathrm{k} \Omega$ to $70 \mathrm{k} \Omega$ depending on the particular sample. Unfortunately, the annealing procedure, also described in section 2.3.3. results in an increase of resistivity by a factor of about three and a slight bending of the characteristic, colored red in figure 6.11. In this context, the high density of metal-impurities inside the $\mathrm{ZnO}$ substrates (see section 2.2.3) combined with an exposure to ambient air may lead to oxidation processes as well as diffusion of nitrogen and hydrogen into the intrinsic oxygen-vacancies of the ZnO-crystal. That way, the number of free charge carriers would have been reduced resulting in an increase of resistivity.

Furthermore, the majority of experiments were performed using a local measurement setup on the basis of single large-area Ag back-contacts covering almost the whole back-side of the substrates. For comparison reasons, additional substrates were equipped with two separate Ag contacts and an appropriate area size. However, due to the liquid form of the conductive silver and a resulting spread out between sample holder and substrate, these kind of contacts usually exhibit a 2-3 times larger area than evaporated Ti/Au contacts. The corresponding I-V characteristics are also shown in figure 6.11.

Right after preparation, fresh Ag contacts exhibit a resistivity of the same order of magnitude but are also accompanied by a non-linear characteristic which is colored blue. After several measurement cycles and a resulting continuous increase in maxi- 

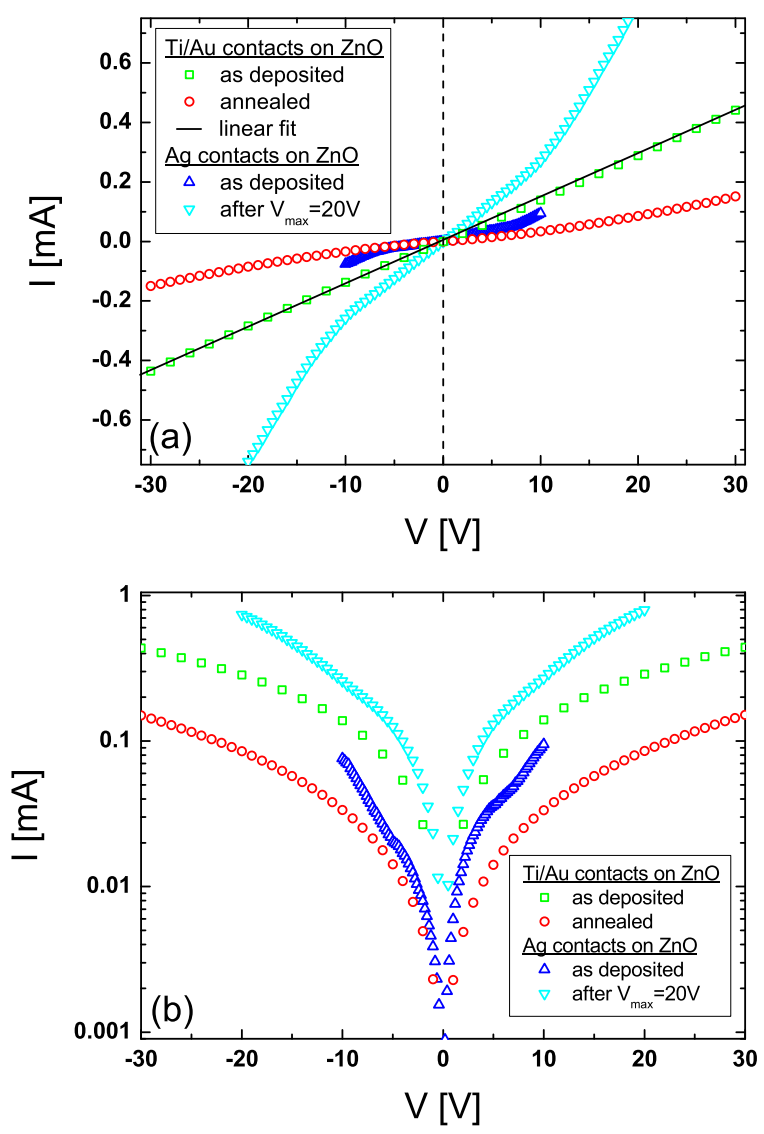

Figure 6.11: I-V characteristics of different back-contact materials on zinc oxide substrates on a linear (a) and on a logarithmic scale (b). The as deposited Ti/Au contacts (green squares) exhibit a linear characteristic indicating ohmic conduction. The corresponding resistance is in the two-digit $\mathrm{k} \Omega$-range. Contrary to theory (section 2.3.3), the annealing procedure results in an increase of resistivity by a factor of about three and a slight bending of the corresponding I-V characteristic (red circles). Fresh Ag contacts exhibit a resistivity of the same order of magnitude as evaporated $\mathrm{Ti} / \mathrm{Au}$ but is also accompanied by a non-linear characteristic (blue triangles). After several measurement cycles and a resulting continuous increase in maximum bias voltage, the conductivity of $\mathrm{Ag}$ contacts increases of about one order of magnitude (teal triangles).

mum bias voltage, the conductivity increases of about one order of magnitude. The corresponding I-V characteristic of $V_{\max }=20 \mathrm{~V}$ is colored teal in figure 6.11. The conductivity is even higher compared to as deposited $\mathrm{Ti} / \mathrm{Au}$ contacts but primarily based on the enlarged contact areas. In addition, the characteristic is not linear. However, the characteristic of large area Ag contacts can be approximated by an ohmic resistor in first order as the main part of this serial resistor is caused by the $\mathrm{ZnO}$ substrates itself. Assembling all of these results into a single conduction model of the whole heterostructure leads to the development of the Frenkel-Poole and ideal diode model (FPID-model), covering all conduction mechanism dominating at different bias voltages ranges based on their point of origin inside the heterostructure 
as well as on the resulting contributions according to the electrical dependencies illustrated in the corresponding equivalent circuit diagram (ECD) of figure 6.10. As the corresponding numerical simulation is in very good agreement with the recorded dataset, the effect of each part of the heterostructure on the electrical properties of the resulting heterojunction diode characteristic can be analyzed with respect to the applied bias voltage or intensity of the applied electrical field, respectively. In principle, the reverse-bias region is dominated by the Schottky diode and the

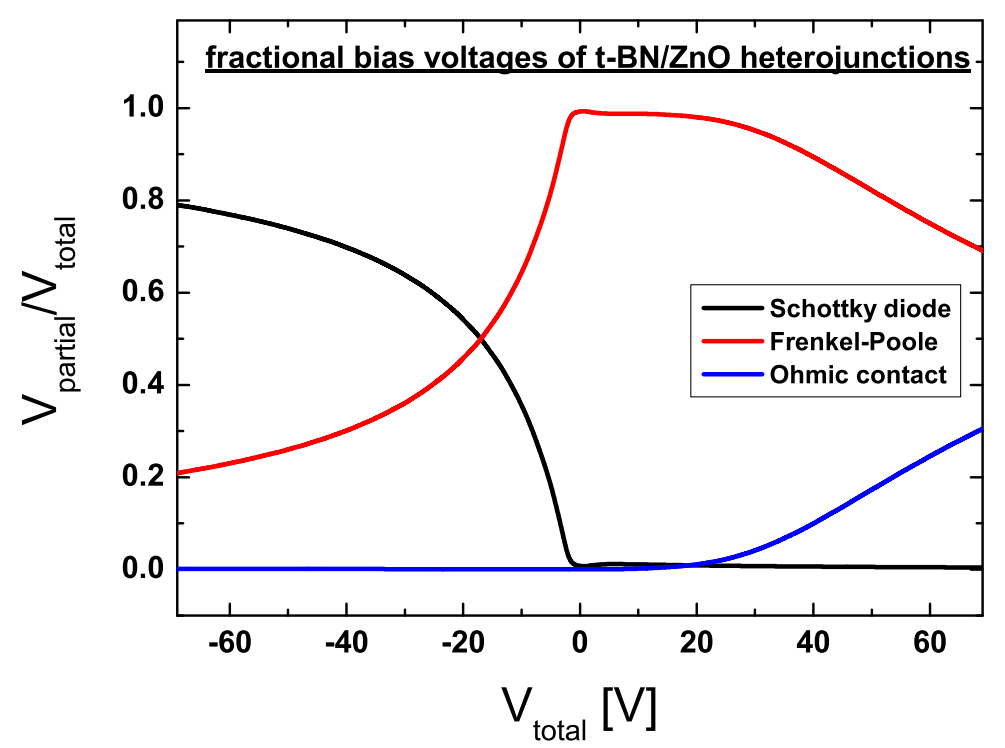

Figure 6.12: Fractional bias voltages of a typical $\mathrm{Au} / \mathrm{Cr} / \mathrm{t}-\mathrm{BN} / \mathrm{ZnO}$ heterojunction at room temperature based on the numerical simulation of the FPID-model in figure 6.9. In principle, the reverse-bias region is dominated by the Schottky diode and the corresponding parasitic resistor $R_{\mathrm{P}}$. The forward-bias region, on the other hand, is basically governed by Frenkel-Poole conduction of the t-BN thin-film and contributions from the ohmic serial resistor at high electric fields.

corresponding parasitic resistor $R_{\mathrm{P}}$. The forward-bias region, on the other hand, is basically governed by Frenkel-Poole conduction of the t-BN thin-film and contributions from the ohmic serial resistor at high electric fields.

This can be elaborated in more detail on the basis of the fractional bias voltages in figure 6.12 regarding the individual components of the FPID-model circuit according to the numerical fit data in figure 6.9.

In the forward-bias region, the increase in the I-V characteristic is almost entirely dominated by a Frenkel-Poole type resistor up to a bias voltage of approximately $25 \mathrm{~V}-30 \mathrm{~V}$. At higher electric fields, the exponential increase in conductivity of the FP-resistor leads to a progressive increase of the voltage drop across the ohmic serial resistor $R_{\mathrm{S}}$. The voltage drop across the t-BN thin-film decreases accordingly. At a bias voltage of $70 \mathrm{~V}$, the ohmic serial resistor provides a significant contribution of about $30 \%$ but becomes even more dominant at higher voltages. For $V \leq 15 \mathrm{~V}$ 
at reverse-bias, the characteristic is also primarily dominated by the FP-resistor. However, the voltage drop progressively shifts towards the Schottky diode D with increasing field intensity. At high electric fields in the reverse-bias region, almost the entire voltage drop $(\geq 80 \%)$ takes place across the Schottky diode including the parasitic resistor $R_{\mathrm{P} 1}$ accompanied by a slight contribution from the FP-resistor of the thin-film $(\approx 20 \%)$.

In order to classify this kind of heterojunction in terms of the Shockley-equation (see eq. 3.12) regarding common $\mathrm{p}-\mathrm{n}$ and Schottky junction diodes similar to the work of several other groups in literature (see chapter 1), a so called non-ideal diode model (NID model) was developed during earlier work [53] prior to the FPID model. In this extended Shockley model, the heterojunction is described by a non-ideal diode with an ideality factor of $n \gg 2$, an ohmic parasitic leakage resistor $\mathrm{R}_{\mathrm{P}}$, arranged parallel to the diode, and a serial ohmic resistor $R_{\mathrm{S}}$ covering additional contact resistances as well as those resistances caused by the substrate. This is illustrated

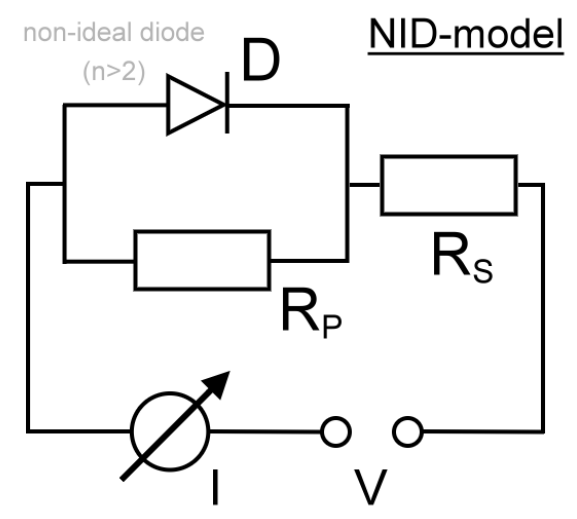

Figure 6.13: Equivalent circuit diagram of the Non-ideal diode model (NID-model): In this extended Shockley model, the heterojunction is described by a serial arrangement of a non-ideal Schottky diode $(n \gg 2)$ and an additional ohmic resistor $R_{\mathrm{S}}$ covering present contact and substrate resistances. An an ohmic parasitic leakage resistor $R_{P}$ of the diode covering present contact and substrate resistances has also been taken into account.

in an appropriate equivalent circuit diagram in figure 6.13 and the corresponding numerical fit is colored red in figure 6.9.

In comparison to the FPID model, the NID-model only provides a good approximation for high electric fields in both directions. For low fields $(|V| \leq 20 V$ in the example at hand) on the other hand, the fits deviate significantly from the recorded datasets, in particular around zero-bias. In this context, the serial as well as the parasitic ohmic resistor are very similar to those of the FPID-model while the obtained apparently high ideality factor of the non-ideal diode is in the range if $n \approx 115-205$, depending on the particular sample, accompanied by saturation currents of $I_{\mathrm{S}} \approx 20 \mathrm{nA}-100 \mathrm{nA}$. Based on the Shockley theory (see section 3.5) these high ideality factors cannot be explained on a physical basis such as extended forms of recombination processes. However, despite exceeding the array of possible ideality factors covered by the Shockley theory, a comparison of the FPID and 
NID-model provides a possible explanation of such apparently high ideality factors reported in literature for a variety of heterojunctions.

Based on the similarities of the simulated datasets, especially and high electric fields, the presence of high defect densities or amorphous regions inside particular heterostructures might also cause interference of thermionic emission processes like Frenkel-Poole with the originally intended diode characteristic. As a result, this interference affects for instance the electrical properties of the whole device by dominating in the forward-bias region of the heterojunction resulting in apparently high ideality factors of the diode. In addition, these apparently high ideality factors can also be estimated from the slope of the high current region $\left(I / I_{S} \gg 1\right)$ of the forward-bias region of Schottky plots according to:

$$
n=\frac{q}{k T} \frac{d V}{d \ln (I)} .
$$

In a final step, temperature dependent I-V measurements of $\mathrm{Au} / \mathrm{Cr} / \mathrm{t}-\mathrm{BN} / \mathrm{ZnO}$ heterostructures were performed in order to verify the validity of the FPID-model and the temperature dependencies of the individual components within.

The corresponding characteristics of a typical sample are shown in figure 6.14 The pronounced rectifying characteristic can be observed down to a temperature of about $160 \mathrm{~K}-170 \mathrm{~K}$. After that, at temperatures around $150 \mathrm{~K}$, the temperature dependence of the characteristics starts to vanish accompanied by an increase in symmetry. Finally, at temperature below $125 \mathrm{~K}$, the I-V characteristics of the t-BN/ZnO system become completely temperature independent and additionally exhibit an almost linear dependence. Considering the residual conductivity of t-BN in this temperature region (see section 6.1.1) as well as the serial arrangement of the individual components in the FPID-model circuit, this behavior might either be caused by insulation effects inside the $\mathrm{ZnO}$ substrate (and/or the $\mathrm{ZnO}$ /back-contact interface) or by reaching the lower noise threshold of the SMU.

Furthermore, the numerical simulations based on the FPID-model are represented by solid lines of the same colors as the corresponding recorded datasets in figure 6.14. In general, these simulations are in very good agreement with the recorded $\mathrm{I}-\mathrm{V}$ characteristics down to temperatures of about $160 \mathrm{~K}$ (175 K in the example at hand). However, at temperatures below $260 \mathrm{~K}$ the measured data show slight discrepancies from the predicted characteristics of the simulation for bias voltages exceeding about $35 \mathrm{~V}-40 \mathrm{~V}$ at reverse-bias indicating slight imperfections of the model, especially regarding ohmic conduction of the parasitic resistor of the diode at all temperatures and field intensities. At approximately $150 \mathrm{~K}$, the simulated data start to deviate from the measured I-V characteristic most notably at low electric fields in the range of about $-10 \mathrm{~V}$ to $+20 \mathrm{~V}$. This behavior can also be linked to the residual conductivity of the t-BN thin-film. As the FPID-model is only valid for a Frenkel-Poole like resistor, the emerging residual conductivity of the t-BN at this

\footnotetext{
${ }^{3}$ In this context, the kinks of the particular characteristics are basically caused by slight hysteresis effects during the measurements and the subsequent averaging process of the data
} 


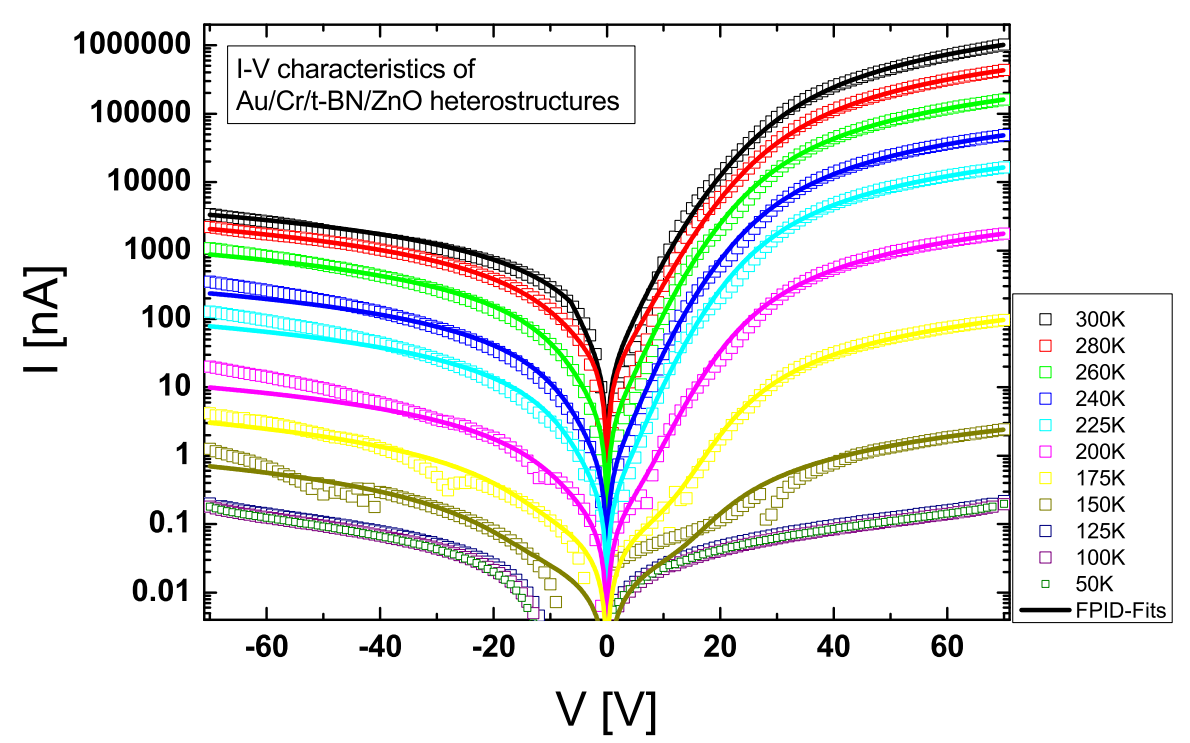

Figure 6.14: Temperature dependent I-V measurements of a typical Au/Cr/t-BN/ZnO heterojunction. The pronounced rectifying characteristics can be observed down to a temperature of about $160 \mathrm{~K}-170 \mathrm{~K}$. Below that, the I-V characteristics become progressively temperature independent accompanied by an increase in symmetry. The numerical simulations based on the FPID model (colored lines) are in very good agreement with the recorded I-V characteristics (colored circles) down to temperatures of about $160 \mathrm{~K}$. At approximately $150 \mathrm{~K}$, the simulated data start to deviate from the measured I-V characteristic most notably at low electric fields due to the residual conductivity of the t-BN thin-film. In addition, the numerical simulations also exhibit slight discrepancies from the measured data at high electric fields in reverse-bias for $T \leq 260 \mathrm{~K}$ indicating slight imperfections of the model regarding ohmic conduction of the parasitic resistor of the diode.

temperature range (see section 6.1.1) leads to deviations between the FPID-model and the recorded data and eventually to the breakdown of the model for $T \leq 125 \mathrm{~K}$. In addition, these observations can be verified by further characteristics of fractional bias voltages as exemplarily illustrated for temperatures of $300 \mathrm{~K}, 240 \mathrm{~K}$ and $200 \mathrm{~K}$ in figure 6.15.

As long as the simulated datasets are in good agreement with the recorded characteristics, the fractional bias voltages remain almost constant at reverse-bias except for very low voltages of $V \leq 10 \mathrm{~V}$. As the diode component is dominated by the diode itself at low and by the parasitic leakage at higher bias voltages, this is most likely caused by the different temperature dependence of the Schottky diode and the FP-resistor leading to a higher contribution of the diode in this region. At forwardbias, the ohmic serial resistor becomes progressively more dominant with decreasing temperature at the expense of FP-conduction. Therefore, the assumption of insulating effects inside the $\mathrm{ZnO}$ crystal at very low temperatures is reasonable based on the gradual and pronounced increase of the serial resistor below $225 \mathrm{~K}$ of up to two orders of magnitude. 


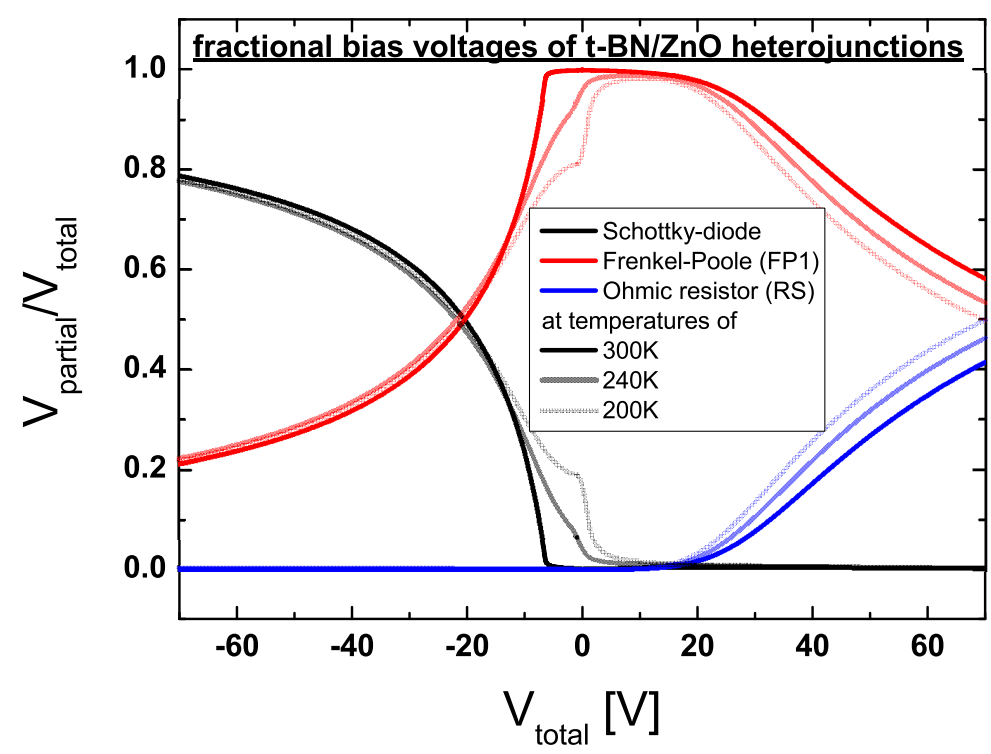

Figure 6.15: Fractional bias voltages of a typical $\mathrm{Au} / \mathrm{Cr} / \mathrm{t}-\mathrm{BN} / \mathrm{ZnO}$ heterojunction at different temperatures of $300 \mathrm{~K}, 240 \mathrm{~K}$ and $200 \mathrm{~K}$ based on the numerical simulations in figure 6.14. As long as the simulated datasets are in good agreement with the recorded characteristics, the fractional bias voltages remain almost constant at reverse-bias except for very low voltages of $V \leq 10 \mathrm{~V}$. At forward-bias, the ohmic serial resistor becomes progressively more dominant with decreasing temperature at the expense of FP-conduction.

In the whole "fitting"-process, the Schottky barrier height $\Phi_{\mathrm{S}}$ has been kept constant at all temperatures similar to the average barrier height $\Phi_{\mathrm{FP}}$ of the FP-resistor which has been taken from the analysis of t-BN reference samples in section 6.1.1, As the Fermi-Dirac distribution of the semiconductor becomes less gradual at lower temperatures [64] accompanied by a decrease in thermal excitation of free charge carriers inside the band-gap, a decrease in barrier height can be excluded. Furthermore, the conductivity $\sigma_{0}$ of the t-BN thin-film inside t-BN/ZnO heterostructures, extracted from the corresponding numerical simulation results in figure 6.14, differs significantly to those of t-BN reference samples in section 6.1.1 by about three orders of magnitude but still exhibits a very similar temperature dependence proportional to $\exp (1 / \mathrm{T})$ as shown in figure 6.16. The strong decrease in conductivity compared to t-BN reference samples likely results from a significantly lowered number of additional free charge carriers provided by the high resistive $\mathrm{ZnO}$ substrate and incorporated in the intermixing process during deposition as described in section 5.3 .1 .

In addition, the remaining free parameters of the FPID-model such as $\zeta \cdot \epsilon_{r}$ of the t-BN film as well as especially the additional resistors $R_{\mathrm{P} 1}$ and $R_{\mathrm{S}}$ each exhibit a significant temperature dependence. In this context, the corresponding resistances increase considerably by several orders of magnitude up to values of about $1 \cdot 10^{9} \Omega$. Based on superposition of the different voltage and temperature dependencies of the 


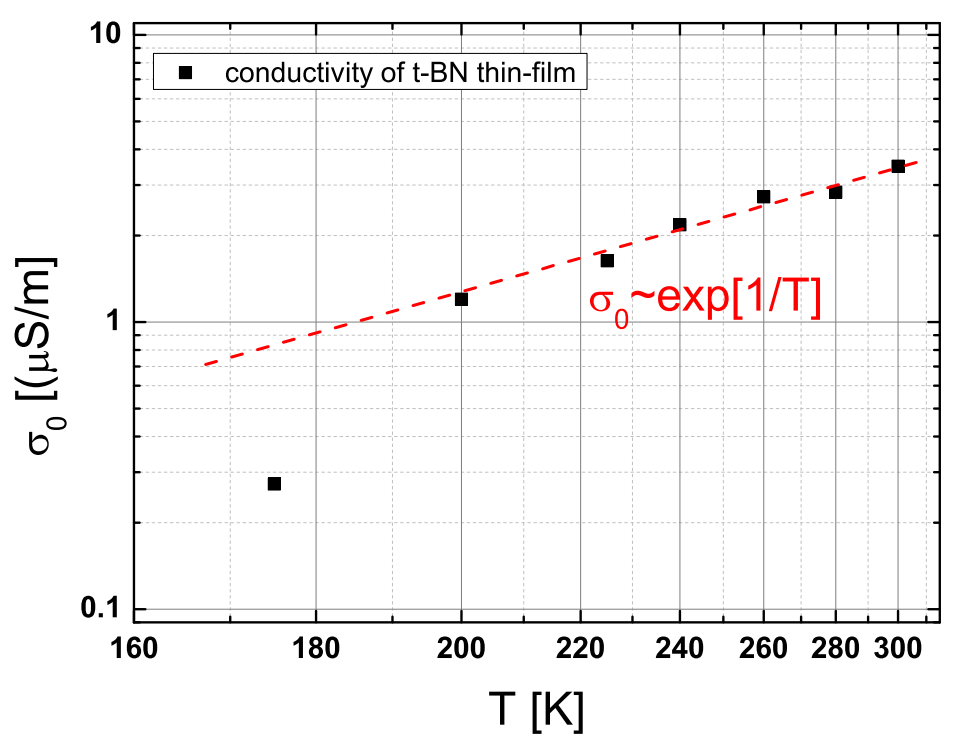

Figure 6.16: Arrhenius-plot regarding the conductivity $\sigma_{0}$ of the t-BN thin-films inside t-BN/ $\mathrm{ZnO}$ heterostructures extracted from the corresponding numerical simulation results in figure 6.14. The conductivity differs significantly by about three orders of magnitude to those of t-BN reference samples in section 6.1.1 but still exhibits a very similar temperature dependence proportional to $\exp (1 / \mathrm{T})$.

individual components, small deviations from the predicted conduction mechanisms cannot be exclusively linked to single parameters of the model. For instance, potentially present non-linearities of anticipated ohmic resistor components at lower temperatures such as slight bending of the corresponding I-V characteristics would be interpreted as a change of curvature $\zeta$ and related to the grown thin-film in terms of the FPID-model regardless of its origin. As a result, the temperature relations of the remaining parameters obtained from the numerical simulations are not entirely conclusive and will therefore not be interpreted any further in this work.

Nevertheless, the present temperature dependence proportional to $\exp (1 / \mathrm{T})$ is consistent with the anticipated behavior of the thin-film component. 


\section{3 ta-C/p-Si heterostructures}

In the next step, the electrical properties of $\mathrm{Au} / \mathrm{Cr} / \mathrm{ta}-\mathrm{C} / \mathrm{p}-\mathrm{Si}$ heterostructures were investigated. A typical I-V characteristic at room temperature (RT) is shown in figure 6.17 . Similar to the t-BN/ZnO heterostructures in section 6.2, the ta-C/pSi system exhibits a pronounced rectifying characteristic of about three orders of magnitude at room temperature. However, the orientation of the diode is inverted. Anticipating the presence of another Schottky diode, this is solely caused by the change in doping type of the semiconductor substrate resulting in a depletion of holes instead of electrons compared to a n-type semiconductor such as $\mathrm{ZnO} 4$. Nevertheless, from a qualitative point of view, the characteristics of the ta-C/p-Si system are very similar to t-BN/ZnO heterojunctions. In addition, the increase in conductivity, especially at forward-bias, is in accordance with the results of the reference samples regarding the comparison between t-BN and ta-C. Therefore, the FPIDmodel (see figure 6.10) was also applied to the $\mathrm{Au} / \mathrm{Cr} / \mathrm{ta}-\mathrm{C} / \mathrm{p}-\mathrm{Si}$ heterojunctions and therefore to another system with different composition but similar structural properties. The corresponding numerical simulation of the FPID-model is additionally illustrated in figure 6.17 by the blue solid line and is in good agreement with the recorded data. Again, in order to limit the number of free parameters inside the FPID-model all essential parameters were determined or alternatively estimated prior to the actual fitting process.

First of all, additional Schottky barriers between the metal top-contacts and the ta-C can also be ruled out based on the results of the ta-C reference samples in section 6.1.2. Furthermore, as neither p- nor n-type conduction could be realized by boron-, nitrogen- and phosphorous-doping during MSIBD-deposition of ta-C [58], the formation of any p-n junction between ta- $\mathrm{C}$ and the silicon is also highly unlikely. Therefore, the assumption of another one-sided p-n or Schottky junction between the $\mathrm{Au} / \mathrm{Cr}$ top-contact and the p-type silicon is reasonable despite the presence of a thick amorphous silicon-layer in some areas of the original Si-wafer surface, discussed and analyzed in section 5.3.5. As Schottky barriers are also formed at the interface between amorphous silicon and several metal contact materials [156], the resulting change of band-gap energy is likely accompanied by a slight shift of diode parameters such as barrier height and effective mass of the majority carriers. Due to the lack of suitable analysis-methods regarding the recombination processes inside the depletion region and pronounced effects of the ideality factor onto the simulated data of FPID-model, the Schottky diode is presumed "ideal" $(n=1)$ for both types of interlayer structures.

The leakage of the diode is still approximated by an ohmic parasitic resistor despite the presence of an additional amorphous interlayer of about $5 \mathrm{~nm}$ thickness covering the surface of all Si-substrates according to the TEM-analysis in section 5.3.5. However, in comparison to the original single crystalline Si-substrate, the presence of the

\footnotetext{
${ }^{4}$ This is discussed in more detail in the end of the section based on similar grown ta-C/Si heterostructures on n-type Si-substrates with identical conductivity.
} 

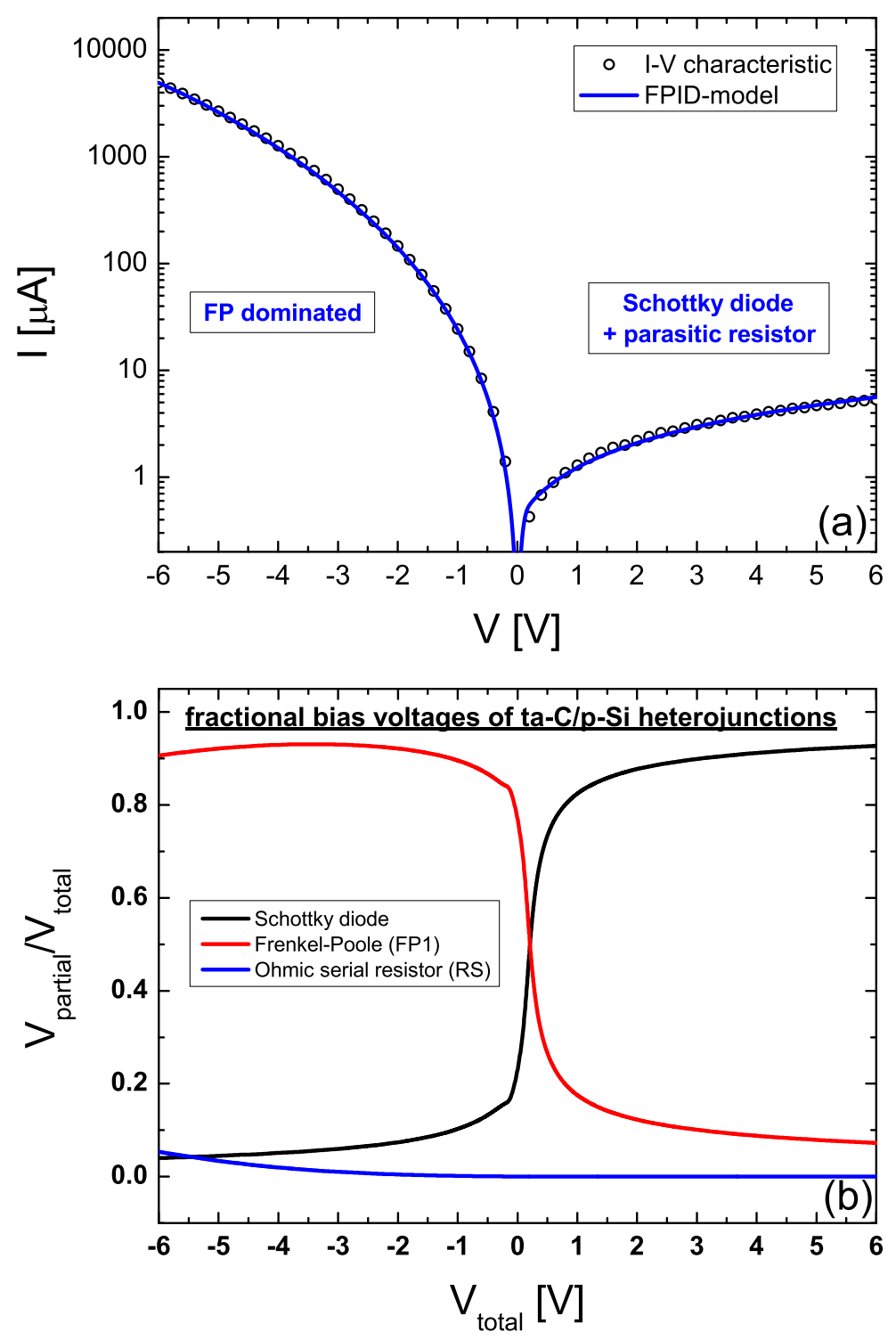

Figure 6.17: (a) I-V characteristic of a typical Au/Cr/ta-C/p-Si heterostructure at $300 \mathrm{~K}$. The ta-C/p-Si system exhibits a pronounced rectifying characteristic of about three orders of magnitude at room temperature. However, the orientation of the diode is inverted compared to $\mathrm{ZnO}$ based samples due the p-type doping of the silicon. The corresponding numerical simulation of the FPIDmodel (blue solid line) is in good agreement with the recorded data despite the presence of the amorphous interlayer according to TEM-measurements in section 5.3.5. (b) Fractional bias voltage characteristic based on the numerical simulation of the FPID-model.

amorphous interlayer, resulting from the sputter-cleaning process, in combination with the implanted carbon ions into its surface most likely leads to an increase of parasitic currents.

The forward-bias direction, on the other hand, is primarily dominated by Frenkel- 
Poole like conduction according to equation 6.1 of the grown ta-C thin-film. Again, based on the TEM-analyses in sections 5.3.3 and 5.3.5, the ta-C thin-film growth on p-type silicon is almost identical to the corresponding ta-C reference samples on n-type silicon. Therefore, the parameters of the corresponding Frenkel-Poole (FP) resistor are predominantly based on the results of the ta-C reference samples in section 6.1.2, especially the average barrier height of $\Phi_{\mathrm{FP}, \mathrm{ta}-\mathrm{C}}=0.25(2) \mathrm{V}$. Similar to t-BN on $\mathrm{ZnO}$, the corresponding leakage current of the FP-resistor is in the pA range and several orders of magnitude lower compared to ta-C reference samples. Therefore, it can also be neglected for this system.

Finally, contact resistances as well as the intrinsic resistance of the semiconducting substrate were analyzed in order to estimate the ohmic serial resistor of the particular heterojunctions of the ta-C/p-Si system. During the scope of this thesis, the quality of back-contacts was continuously optimized for these substrates. In the process, different back contact materials such as Ag, similar to the ta-C reference samples, and Al-back-contacts according to section 2.3.1 in an as deposited as well as an annealed state were used. Therefore, the electrical properties of the different contact materials were investigated. The corresponding I-V characteristics of 2 separate back contacts each are summarized in figure 6.18 on a linear (a) as well as on a logarithmic scale (b). The as deposited $\mathrm{Al}$ contacts (colored red) exhibit a high resistance in the two-digit $\mathrm{k} \Omega$-range and a S-shaped curvature which is likely caused by some kind of Schottky barrier formation at the interface between $\mathrm{Al}$ and Si. Nevertheless, after annealing at $900^{\circ} \mathrm{C}$, the resistivity decreases by more than one order of magnitude at low electric fields and the resulting I-V characteristic (colored green) is almost perfectly linear indicating ohmic conduction. Based on the large distance of the back-contacts (see figure 2.7) compared to the actual substrate thickness of $625 \mu \mathrm{m}$, the actual serial ohmic resistor of the FPID-model is presumably even further reduced by at least one order of magnitude.

On the other hand, Ag back-contacts (colored teal) always exhibit a non-linear, Sshaped characteristic likely caused by some kind of Schottky barrier formed between $\mathrm{Ag}$ and $\mathrm{Si}$. Nevertheless, if the $\mathrm{Ag}$ is applied to the Si right after scratching the back-side of the substrates, the resulting contact resistance is almost identical to annealed Al-contacts. At bias voltages exceeding approximately $4 \mathrm{~V}$, the Ag contacts exhibit even lower resistances compared to the well defined, low ohmic Al-contacts. However, similar to $\mathrm{ZnO}$ substrates, due to the liquid form of the conductive silver and a resulting spread out between sample holder and substrate, each of these contacts is usually also 2-3 times larger than evaporated contacts leading to an increase in current. In addition, after a period of several days, these kind of contacts start to degrade resulting in an increase of resistivity and even in slight asymmetric characteristics (colored blue). As the solvent of the liquid Ag vaporizes over time, small fissures inside the back-contact material might lead to a progressing oxidation of the silicon surface eventually leading to high resistive back-contacts after some time (months).

During the actual investigation of the ta-C/p-Si heterojunctions, the high resistiv- 

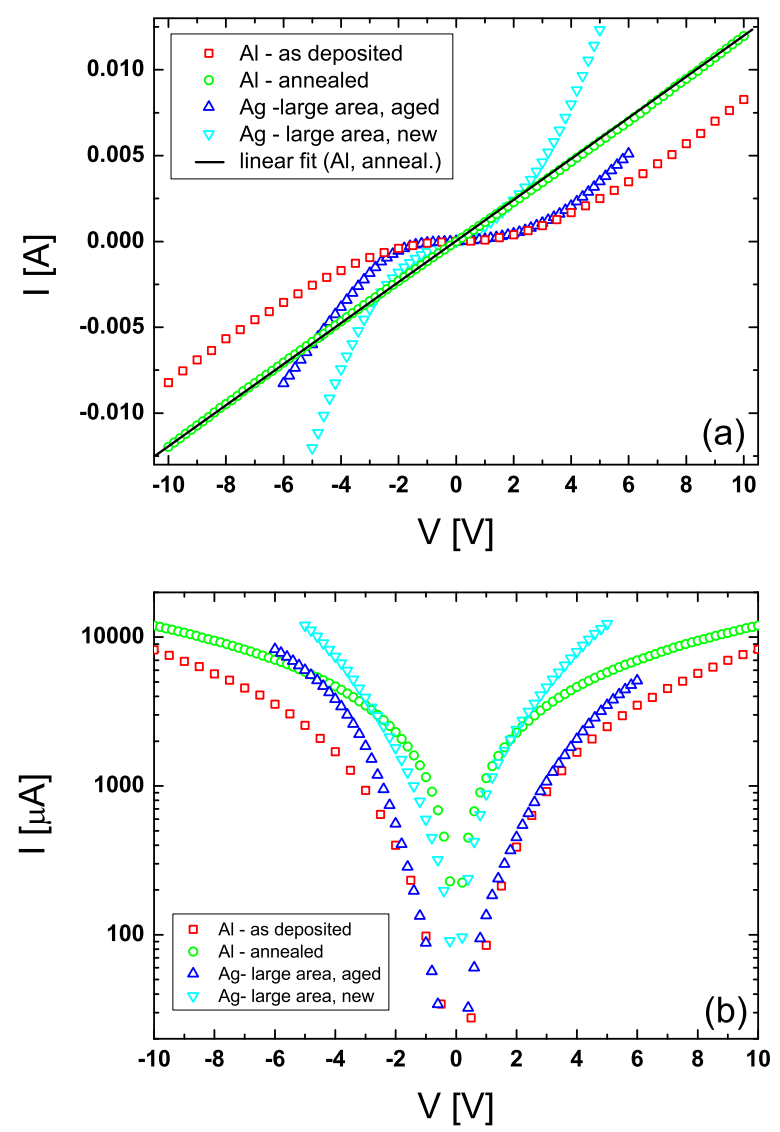

Figure 6.18: I-V characteristics of different back-contact materials on p-type silicon substrates. As deposited $\mathrm{Al}$ contacts (colored red) exhibit high resistances in the two-digit $\mathrm{k} \Omega$-range and a $\mathrm{S}$ shaped curvature likely caused by some kind of Schottky barrier formation at the interface between $\mathrm{Al}$ and $\mathrm{Si}$. After annealing at $900^{\circ} \mathrm{C}$, the resistivity decreases by more than one order of magnitude at low electric fields and the resulting I-V characteristic (colored green) is almost perfectly linear indicating ohmic conduction. The resistance of about 1 mathrmk $\Omega$ is primarily based on the resistivity of the p-Si substrate. Fresh Ag back-contacts (colored teal) always exhibit a non-linear, S-shaped characteristic likely caused by Schottky barrier formation but also low resistances at higher electric fields. However, after a period of several days, these kind of contacts start to degrade resulting in an increase of resistivity (colored blue).

ity of the $\mathrm{Ag}$ and as deposited $\mathrm{Al}$ contacts was compensated by a remote setup of the samples. In contrast, ta-C/p-Si heterostructures exhibiting annealed Al backcontacts were investigated in remote and also local configuration setup, especially during photosensitive (see chapter 7) and frequency dependent measurements (see chapter 8).

Focusing on the numerical simulation in figure 6.17a according to the FPID-model, the fractional bias voltages again provide detailed information about the electrical properties of the individual components of the MASS diode structure as well as their interaction and are shown in figure 6.17b. In this context, the corresponding struc- 
tural properties based on the TEM-analysis in section 5.3.5 have also been taken into account.

At forward-bias, the major voltage drop is across the ta-C thin-film $(\approx 90 \%)$ with a slight but progressively decreasing contribution from the Schottky diode. At higher voltages $(\geq 3 \mathrm{~V})$, there is also a slight contribution from the serial resistor which is progressively increasing at higher electrical fields. The serial resistor primarily originates from the silicon substrate itself as measurements were performed in remote configuration eleminating the back-contact resistances almost entirely. At low bias voltages in the reverse-bias region $(V \leq 1 \mathrm{~V})$, the I-V characteristic is also significantly affected by the FP-resistor (70 \%-10\%, gradually decreasing). At higher elelectric fields, almost the complete voltage drop takes place across the Schottky diode including the parasitic resistor $R_{\mathrm{P} 1}(\geq 90 \%)$. On a side-note, the characteristic in forward-bias direction exhibits an apparently high ideality factor of $n \approx 25$ estimated by equation 6.6 in terms of the NID-model.

In order to analyze the stability and possible breakdown thresholds of the heterojunctions, additional measurements were performed at significantly higher maximum bias voltages around RT and ambient conditions. None of the performed measurements showed any sign of electrical breakdown up to reverse-bias voltages of about $125 \mathrm{~V}$. Due to a slight top-contact degradation due to partial oxidization of the Cr-layer, these kind of measurements were only performed on a few samples. A typical I-V characteristic including a numerical simulation of the recorded data with respect to the FPID-model as well as the corresponding fractional bias voltages is illustrated in figure 6.19.

In general, the rectifying effects of the heterojunction diode increase even further at high currents to about four orders of magnitude. The forward-bias direction, in this context, is even limited to a value of just $16 \mathrm{~V}$ due to the compliance of the SMU of $0.1 \mathrm{~A}$.

At forward-bias, the simulation matches the recorded data almost perfectly as expected from the previous measurements at lower voltages shown in figure 6.17. At reverse-bias voltages exceeding about $30 \mathrm{~V}$, the simulated data overestimate the actual current values of the recorded I-V characteristic leading to a progressively increasing deviation of up to about $50 \%$ at higher electric fields. Therefore, the saturation process of the heterojunction diode is more distinct at high field-intensities than predicted by the FPID-model. In this context, possible causes are a voltage dependent barrier height of the Schottky diode or a voltage dependent parasitic leakage current of the diode inversely proportional to the applied bias voltage.

Nevertheless, considering the simplicity of the individual components of the FPIDmodel, the numerical simulation is still in good agreement with the recorded data. In addition, focusing on the corresponding fractional bias voltages, the results are in accordance with those shown in figure 6.17b. As predicted, the ohmic serial resistor becomes more dominant at higher bias voltages at forward-bias. Furthermore, at reverse-bias voltages above $100 \mathrm{~V}$ the voltage drop across the Schottky diode including the parasitic resistor $R_{\mathrm{P} 1}$ has a value of nearly $99 \%$ despite of the discrepancies 

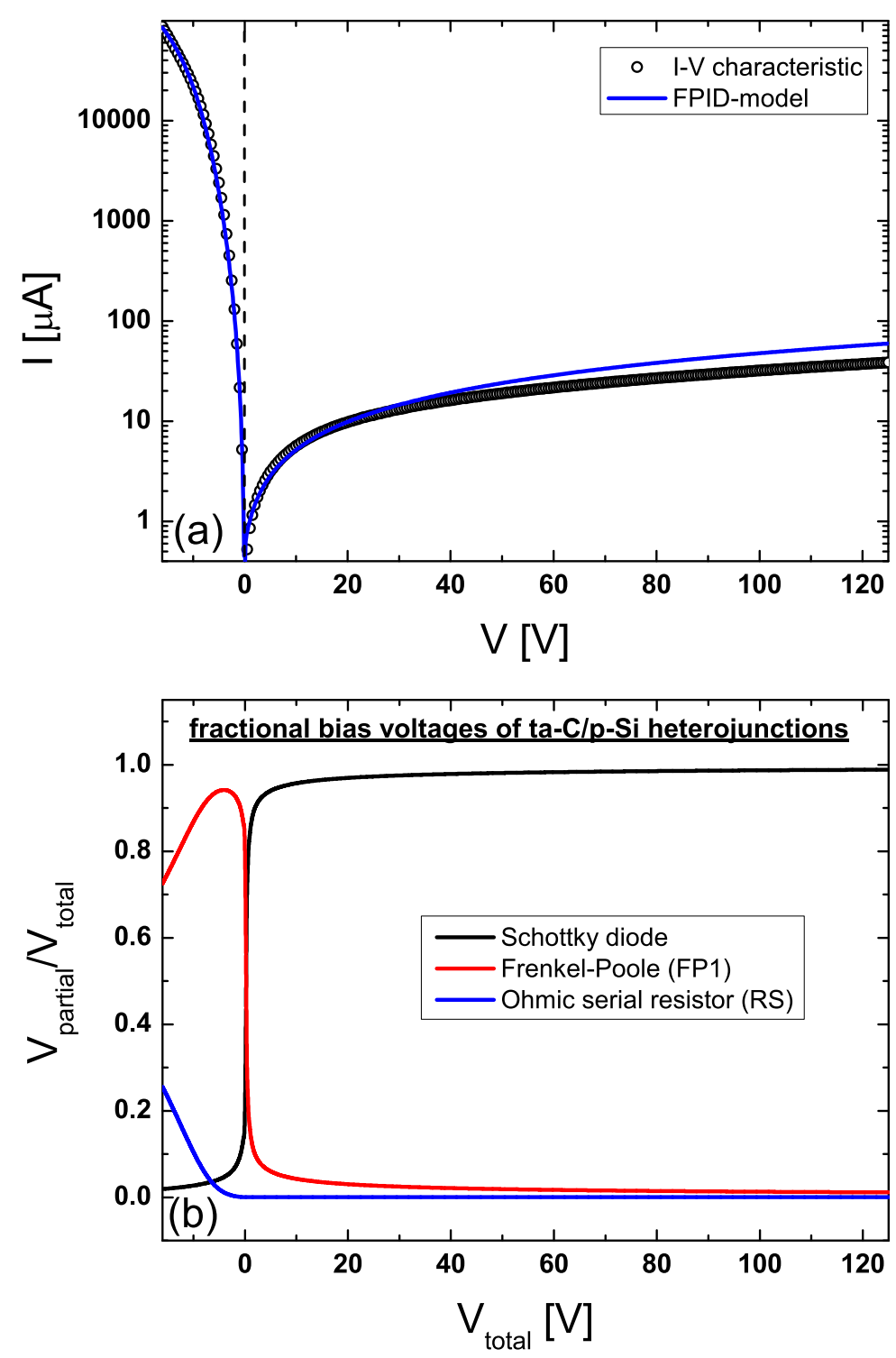

Figure 6.19: (a) I-V measurement of a typical $\mathrm{Au} / \mathrm{Cr} / \mathrm{ta}-\mathrm{C} / \mathrm{p}-\mathrm{Si}$ heterojunction at high electric fields. At forward-bias, the simulation matches the recorded data almost perfectly as expected from the previous measurements at lower voltages shown in figure 6.17 At reverse-bias voltages exceeding about $30 \mathrm{~V}$, the simulated data slightly overestimate the actual current values of the recorded I-V characteristic leading to a progressively increasing deviation of up to about $50 \%$ at higher electric fields. Nevertheless, the numerical simulation is still in good agreement with the recorded data considering the simplicity of the individual components of the FPID-model.

(b) Fractional bias voltage characteristic based on the numerical simulation of the FPID-model. 
between the recorded data and the simulation caused by approximation issues of the component itself in this region.

Finally, the validity of the FPID-model with respect to the ta-C/p-Si MASS diodes was verified by additional temperature dependent $\mathrm{I}-\mathrm{V}$ measurements in the range of $300 \mathrm{~K}-100 \mathrm{~K}$ and corresponding numerical simulations. The resulting datasets of a typical heterostructure are summarized in figure 6.20 including additional fractional bias voltage characteristics at temperatures of $300 \mathrm{~K}, 260 \mathrm{~K}$ and $220 \mathrm{~K}$. All recorded characteristics exhibit a pronounced rectifying characteristic. Due to a higher temperature dependence of the reverse-bias region of the heterojunction compared to the FP-dominated forward-bias direction, the rectifying effect of the diode is increasing at lower temperatures. The numerical simulations according to the FPID-model are in very good agreement with the recorded I-V characteristics down to temperatures of about $180 \mathrm{~K}-160 \mathrm{~K}$.

In this context, the corresponding temperature dependence of the ta- $\mathrm{C}$ conductivity $\sigma_{0}$, extracted from the numerical simulations in figure 6.20, is again proportional to $\exp (1 / \mathrm{T})$ as shown in figure 6.21 and therefore consistent with the results of previous sections. However, compared to ta-C reference samples, the values of $\sigma_{0}$ are about one order of magnitude lower compared to ta-C reference samples on metallic Si which might also be caused by the growth process similar to the discrepancies observed for t-BN based samples in section 6.2. Furthermore, the ohmic resistors $R_{\mathrm{P} 1}$ and $R_{\mathrm{S}}$ again exhibit a pronounced temperature dependence and increase by several orders of magnitude in this temperature range.

At around $160 \mathrm{~K}-140 \mathrm{~K}$, the numerical simulations start to deviate from the recorded datasets in the forwards-bias region as well as at low bias voltages in the reverse-bias region.

In contrast, the recorded dataset of the reverse-bias region is basically in good agreement with the predicted characteristics of the FPID-model as well as the corresponding temperature dependence of the diode. In this context, the related temperature dependence of the parasitic resistor $R_{\mathrm{P} 1}$ is very similar to its equivalent of the $\mathrm{t}$ $\mathrm{BN} / \mathrm{ZnO}$ system (see figure 6.14). Based on the fractional bias voltage analyses in figures 6.17 and 6.19, the discrepancies are predominantly present in bias regions dominated by Frenkel-Poole conduction. In addition, the critical temperature range is almost identical to the transition region and starting point of the residual conductivity of the ta-C reference samples in section 6.1.2. As a result, the failure of the FPID-model with respect to the temperature dependence can be linked to a change of the dominant conduction mechanism inside the grown ta-C thin-films due to the arising residual conductivity of ta- $\mathrm{C}$ in this temperature region (see section 6.1.2). The comparison of fractional bias voltage characteristics at different temperatures of $300 \mathrm{~K}, 260 \mathrm{~K}$ and $220 \mathrm{~K}$ (see figure 6.20) provides further information about the effect of different temperatures on the individual components inside the MASS heterojunction structure.

At high electric fields in forward-bias direction, the voltage drop across all elements of the ECD is almost temperature independent and therefore constant. However, 

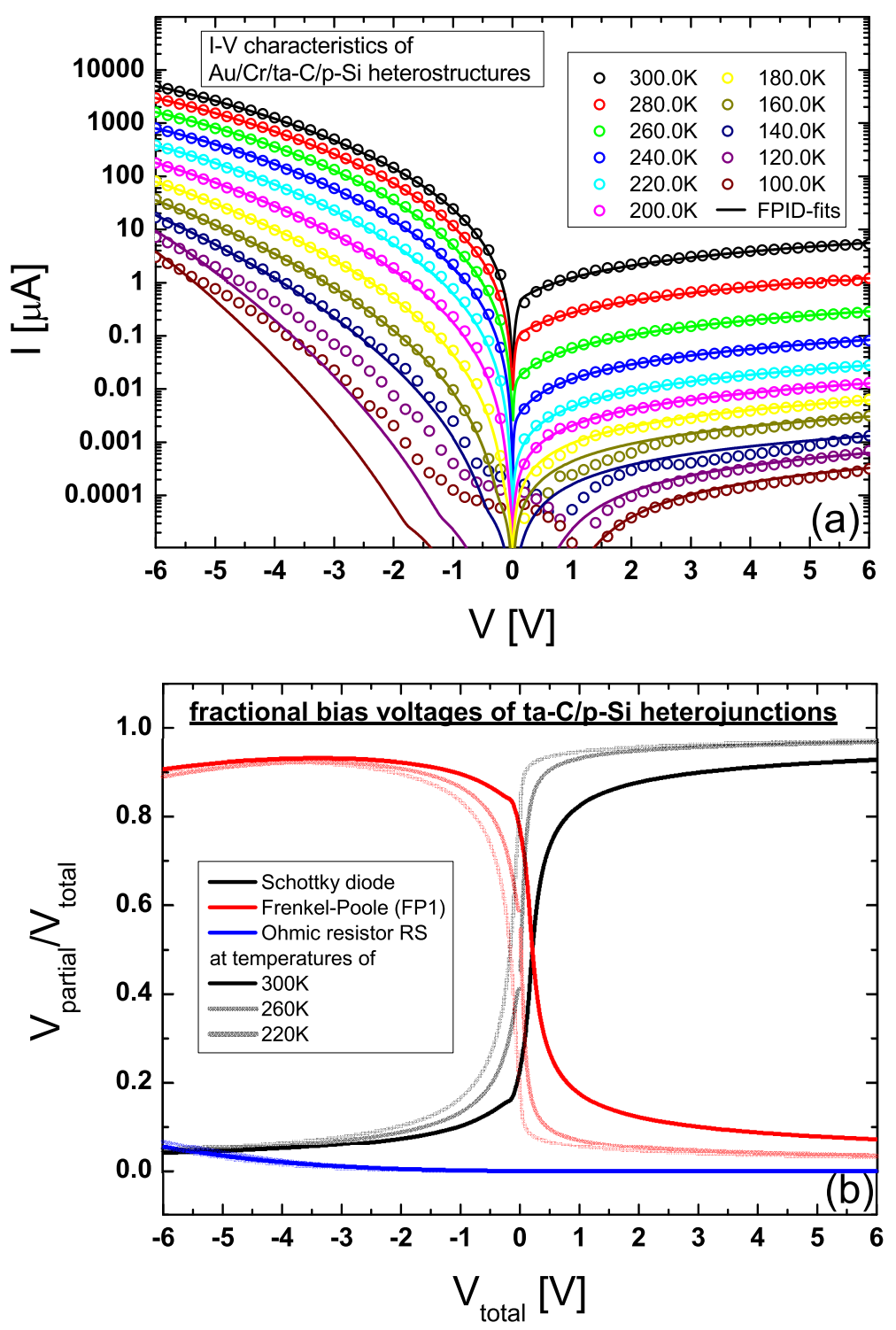

Figure 6.20: (a) Temperature dependent I-V measurements of a typical Au/Cr/ta-C/p-Si heterojunction (colored circles). All recorded characteristics exhibit a pronounced rectifying characteristic. Due to a more pronounced temperature dependence of the reverse-bias region of the heterojunction compared to the FP-dominated forward-bias direction, the rectifying effect of the diode is increasing at lower temperatures. The numerical simulations according to the FPIDmodel (colored solid lines) are in very good agreement with the recorded I-V characteristics down to temperatures of about $180 \mathrm{~K}-160 \mathrm{~K}$. Below that temperature, the numerical simulations start to deviate from the recorded datasets in the forwards-bias region as well as at low bias voltages in the reverse-bias region due to the arising residual conductivity of ta- $\mathrm{C}$ in this temperature region. (b) Comparison of fractional bias voltage characteristics at different temperatures of of $300 \mathrm{~K}$, $260 \mathrm{~K}$ and $220 \mathrm{~K}$. 


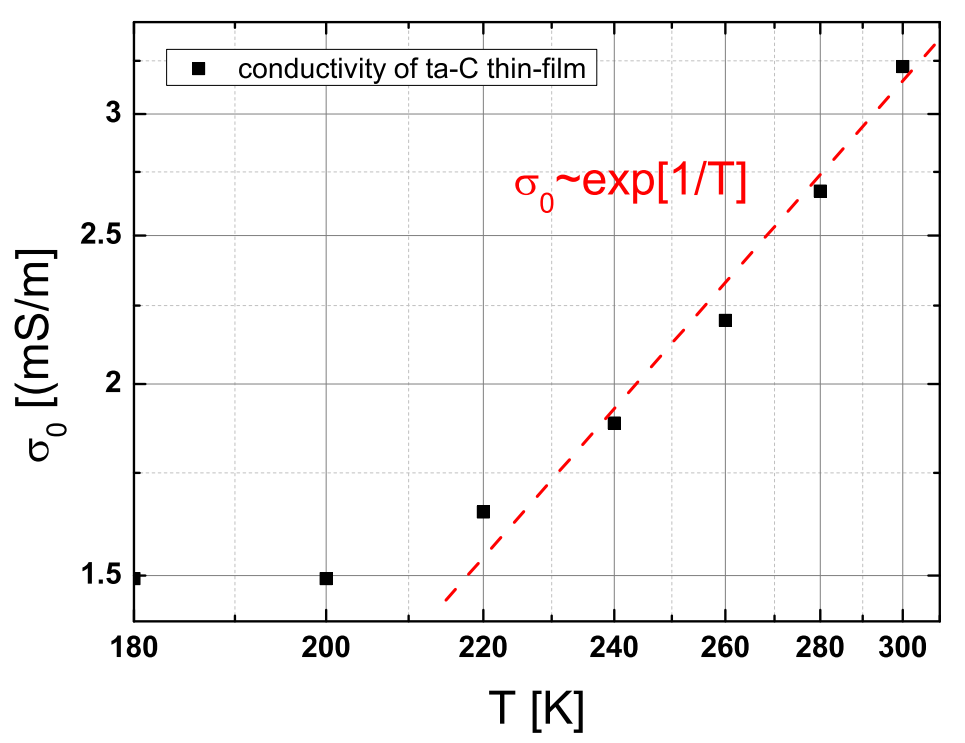

Figure 6.21: Arrhenius-plot regarding the conductivity $\sigma_{0}$ of the ta- $\mathrm{C}$ thin-films inside ta-C/p-Si heterostructures extracted from the numerical simulations in figure 6.20. Again, the conductivity is proportional to $\exp (1 / \mathrm{T})$ but about one order of magnitude lower compared to ta-C reference samples on metallic Si.

for the rest of applied voltages $(V \geq-3 \mathrm{~V})$ a significant shift of voltage drop towards the Schottky diode and the corresponding parasitic leakage in expense of the Frenkel-Poole resistor can be observed at decreasing temperatures, especially between $300 \mathrm{~K}$ and $260 \mathrm{~K}$. As a result, the transition between FP-resistor and Schottky diode becomes distinctively more abrupt around zero-bias and the voltage drop almost entirely takes place across the diode-component in reverse-bias direction of the heterojunction $(\approx 95 \%)$. Therefore, interference of the FP-resistor is almost negligible in this bias-region at lower temperatures.

In general, the observed shift in fractional bias voltages is caused by the different temperature dependencies of the separate regions of the heterojunction, primarily between the Frenkel-Poole dominated thin-film and the depletion region inside the semiconducting substrate. 


\subsubsection{Comparison of different top-contact materials}

During the investigation of the ta-C/p-Si system, one of the samples was additionally equipped with several Al top-contacts in order to investigate the effect of the top contact composition onto the characteristic of the heterojunction, especially onto the region dominated by the Schottky diode.

They were evaporated in a homogeneous area with minimal variation of film-thickness right next to the $\mathrm{Au} / \mathrm{Cr}$ contact pads and exhibit an identical diameter. As they were not annealed to prevent any damage to the grown heterostructure, a formation of some kind of Schottky contact is expected according to theory (see section 2.3.1). However, the adhesion of Al-films is relatively low compared to $\mathrm{Au} / \mathrm{Cr}$ leading to

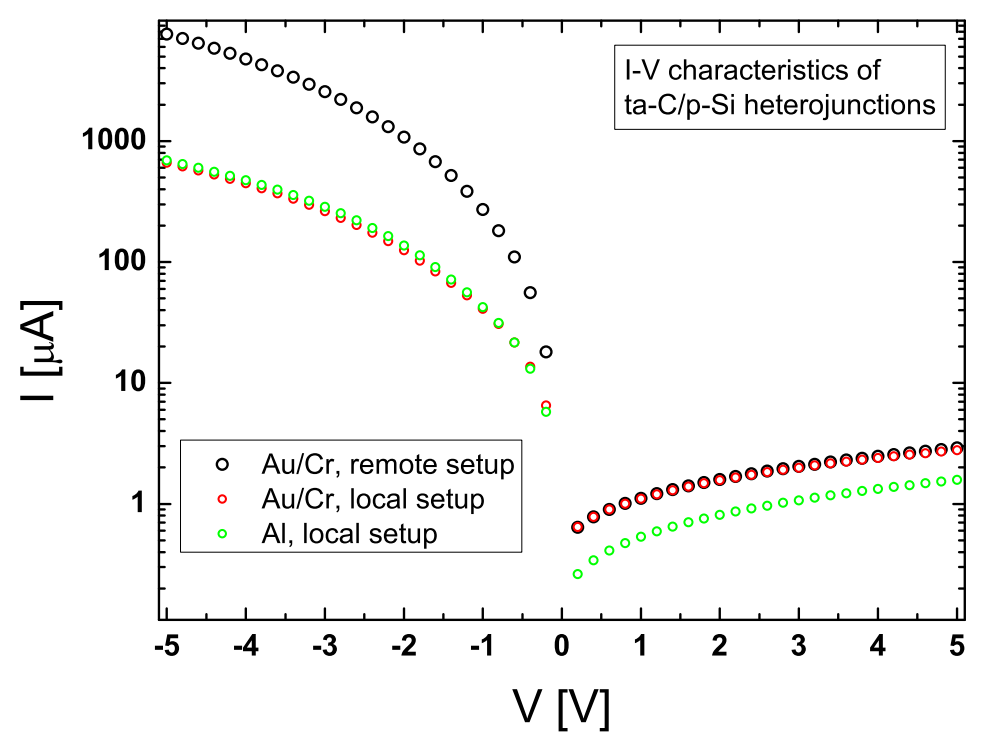

Figure 6.22: $\mathrm{I}-\mathrm{V}$ characteristics of $\mathrm{Al}$ and $\mathrm{Au} / \mathrm{Cr}$ top-contacts on ta-C/p-Si heterostructures recorded in a local measurement setup. Compared to a (pseudo-) remote measurement using a $\mathrm{Cr} / \mathrm{Au}$ top-contact (black circles), the effect of non-annealed Al back-contacts onto the I-V characteristic (red circles) is only limited to the forward-bias direction. Focusing on the local measurements of the different top-contact materials, the recorded I-V characteristics are almost identical at forward-bias indicating no dependency of the Frenkel-Poole like conduction mechanism on contact composition and corresponding interface for constant film-thicknesses. At reverse-bias, on the other hand, the characteristics differ significantly by a factor of about 2 indicating an increased barrier height of Al-contacts of approximately $4 \%$ compared to $\mathrm{Au} / \mathrm{Cr}$ accompanied by a reduction of the parasitic leakage current of about $50 \%$ based on FPID-model simulations.

delamination effects of the evaporated contacts. As a result, only one half of them (3 out of 6 ) were stable and could be bonded properly but only once.

The corresponding I-V characteristics of both contact-types are shown in figure6.22. As both $\mathrm{I}-\mathrm{V}$ measurements (Al: green, $\mathrm{Au} / \mathrm{Cr}$ : red) were only recorded in a local measurement setup, they are additionally compared to a (pseudo-) remote measurement (black circles) of the same $\mathrm{Au} / \mathrm{Cr}$ top-contact to illustrate the effect of contact 
resistances on the I-V characteristics of MASS heterojunctions. In this context, the I-V characteristics of the local measurement setup exhibit a much lower conductivity compared to the remote setup. However, the effect is only limited to the forwardbias direction as these samples were equipped with "as deposited", non-annealed Al back-contacts.

Combining the results of the fractional bias voltage analyses in figures 6.17 and the electrical properties of these contacts, shown in figure 6.18, the discrepancy is solely based on non-linear $\mathrm{I}-\mathrm{V}$ characteristic of the back-contact in the $\mathrm{k} \Omega$ range and not related to the ta-C thin-film itself or top-contact properties.

Focusing on the local measurements of the different top-contact materials, the recorded I-V characteristics are almost identical at forward-bias. As predicted by theory, the Frenkel-Poole like conduction mechanism is only proportional to the contact area but does not depend on top-contact composition and corresponding interface for constant film-thicknesses.

At reverse-bias, on the other hand, the characteristics differ significantly by a factor of about 2. Based on FPID-model simulations, this is equal to an increased barrier height of Al-contacts of approximately $4 \%$ compared to $\mathrm{Au} / \mathrm{Cr}$ accompanied by a reduction of the parasitic leakage current of about $50 \%$. In the absence of any other differences in structural properties and composition of the MASS heterostructure, the discrepancies at reverse-bias can be linked entirely to the difference between the two work-functions of the metal top-contacts and the corresponding difference in barrier heights. Due to the lack of a pure Schottky contact between the semiconductor and the evaporated metal, any comparisons of work-functions or barrier heights to literature data would only be highly speculative.

\subsubsection{Comparison with ta-C/n-Si heterostructures}

In a last step, in order to verify the presence of an one-sided p-n junction inside MASS diode heterostructures, the results of the ta-C/p-Si system are compared to the results of previous experiments based on ta-C/Si heterostructures grown on ntype silicon. The n-type doped Si substrates were also single-side polished, (100) oriented and had a resistivity of $5-14 \Omega \mathrm{cm}$. Therefore, they are comparable to the p-type silicon used in this work. The thin-films were grown inside the same ionaccelerator setup (see section 2.5) and feature an estimated film thickness of about $80 \mathrm{~nm}$ based on the calculations using equation 2.1] and the results of the TEManalyses in chapter 5. Electrical back contacts to Si were realized using conductive silver. Al contacts with a diameter of $d=0.4 \mathrm{~mm}$ were evaporated on top of the ta-C surface. The connections to the SMU (identical to the one used in this thesis) were realized using pressure contacts made of osmium.

The original datasets were recorded and published by Ronning et al. [57], but reanalyzed in the scope of this thesis in terms of the MASS diode structure and the corresponding development of the FPID-model [149]. The I-V characteristic of a typical sample as well as corresponding numerical simulations of the FPID- and the 


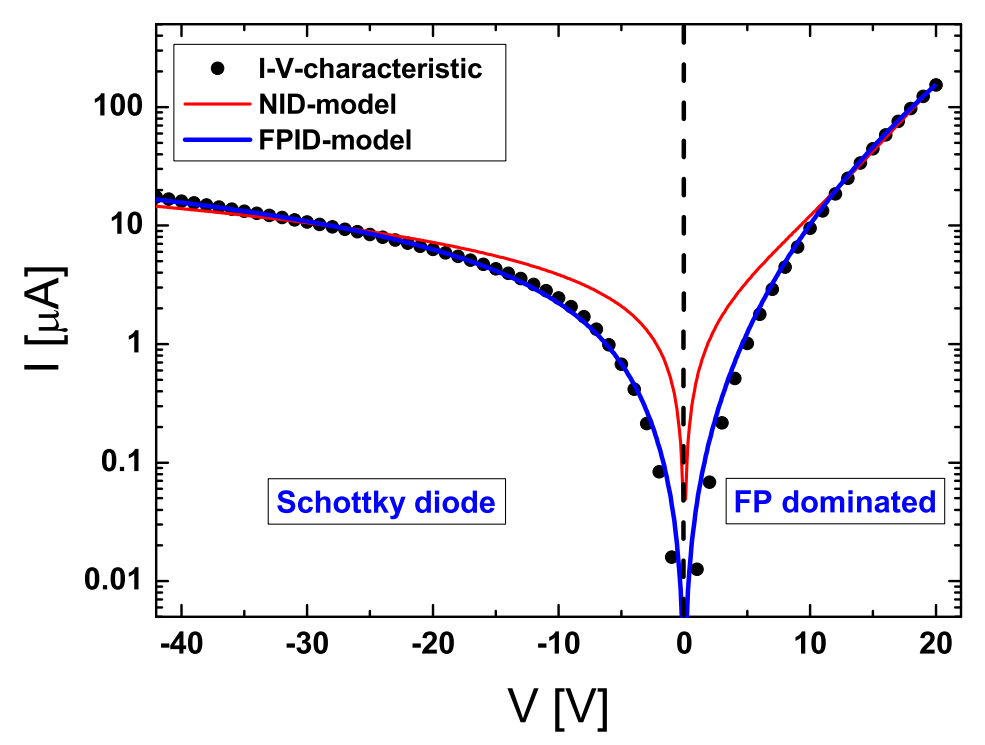

Figure 6.23: $\mathrm{I}-\mathrm{V}$ measurement of a typical $\mathrm{Al} / \mathrm{ta}-\mathrm{C} / \mathrm{n}-\mathrm{Si}$ heterojunction (black circles) at room temperature. The heterojunction exhibits a pronounced rectifying characteristic as well as similar parasitic currents at reverse-bias and an inverse polarity compared to p-Si based samples. The numerical simulation of the FPID-model (blue solid line) is in good agreement with the recorded dataset while the NID-model simulation (red solid line) is only valid for high electric fields $(n \approx 80)$.

NID-model are shown in figure 6.23.

The heterojunctions exhibit a pronounced rectifying characteristic and similar parasitic currents at reverse-bias compared to p-Si based samples. However, in comparison to ta-C based MASS diodes on p-type silicon the diode of the ta-C/n-Si system exhibits inverse polarity. Based on the similar composition of the two systems such as an identical deposition technique, similar contact materials (mentioned in section 6.3.1), resistivity and orientation of the Si-substrates as well as similar parasitic currents of both systems, the opposite polarities of the particular diodes can only be linked to the type of doping regarding the Si-substrates. This is consistent with the formation of Schottky barriers between the evaporated metal contacts and the semiconducting substrates according to theory, described in section 3.6.

In addition, the FPID-model can successfully be applied to these heterojunctions. The corresponding characteristic is colored blue in figure 6.23 and in good agreement with the recorded dataset while the NID-model simulation (red solid line) is again only valid for high electric fields $(n \approx 80)$. As the measurements were only recorded in a local measurement setup, the resulting parameters of the FP-resistor obtained in forward-bias direction as well as a precise value of the serial resistor are just estimates of the actual values. Nevertheless, at least the contact resistance can be estimated to a value of several $\mathrm{k} \Omega$ and the average barrier height of the FP-like resistor with a value of $\Phi_{\mathrm{FP}}=0.245$ is still valid.

Furthermore, the numerical simulation leads to a rough estimate of the correspond- 
ing fractional bias voltages as shown in figure 6.24. In the forward-bias region, the major voltage drop is across the ta-C film, modeled by a Frenkel-Poole type resistance. In addition, there are slight contributions from the ohmic contact resistance (a few hundred Ohm) and the ideal Schottky diode. At high reverse-bias, almost the complete voltage drop takes place across the Schottky diode (including parasitic resistance $\left.R_{\mathrm{P} 1}\right)$.

Therefore the I-V characteristic of a MASS diode is governed by the (ideal) Schottky barrier at reverse-bias and by Frenkel-Poole conduction of the disordered film at forward-bias similar to the ta-C/p-Si system.

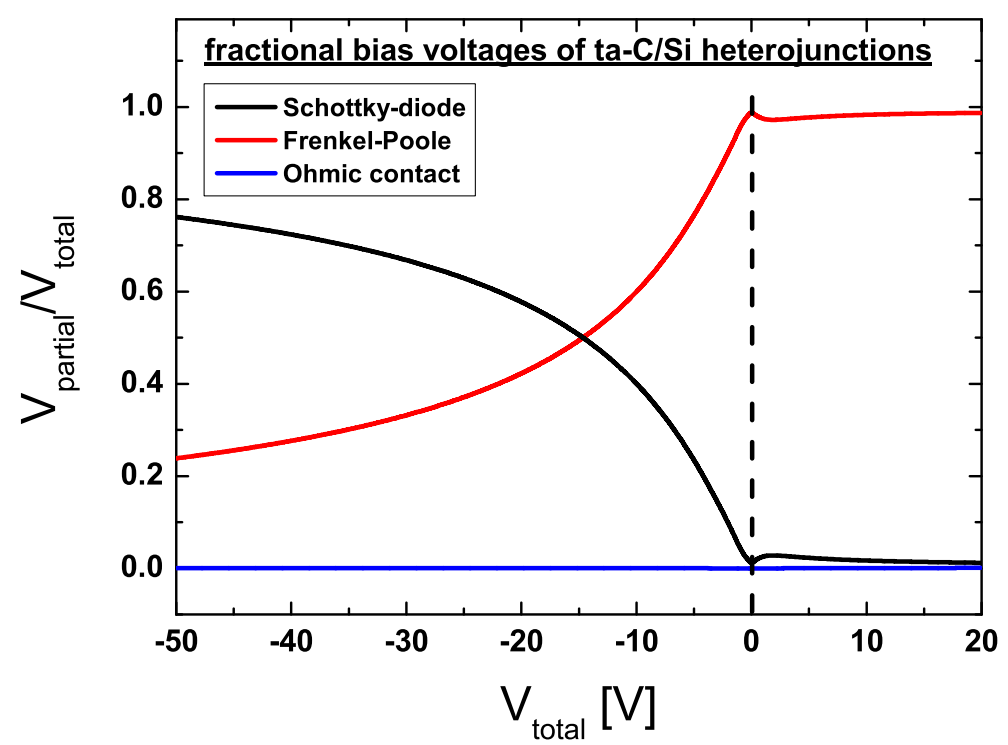

Figure 6.24: Fractional bias voltages of an $\mathrm{Al} / \mathrm{ta}-\mathrm{C} / \mathrm{n}-\mathrm{Si}$ heterojunction at room temperature based on the numerical simulation of the FPID-model in figure 6.23 


\subsection{SiC-based MASS diodes}

In a further step, $\mathrm{SiC}$ based MASS heterostructures were investigated with respect to their electrical properties. As described in section 2.3.2, most of the samples were equipped with annealed $\mathrm{Ni}$ contacts. However, some of them were only equipped
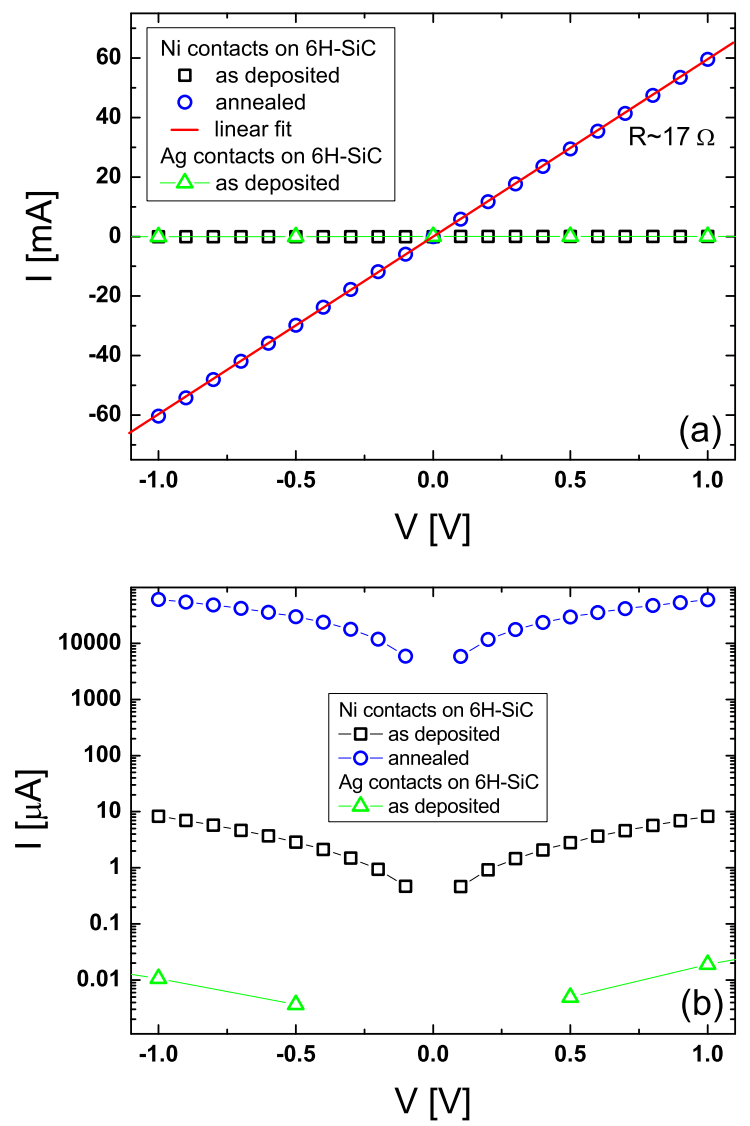

Figure 6.25: $\mathrm{I}-\mathrm{V}$ characteristics of different back-contact materials on $6 \mathrm{H}$ silicon carbide substrates on a linear (a) and and log scale (b). Ag back-contacts (colored green) exhibit very high resistances of about $10^{8} \Omega$ likely caused by the formation of Schottky barriers at the interface of the $\mathrm{SiC}$ substrates. The as deposited pure $\mathrm{Ni}$ back contacts lead to a decrease in resistivity of about three orders of magnitude. The subsequent annealing process eventually results in another significant increase of conductivity of approximately four orders of magnitude and an almost perfect linear I-V characteristic (blue circles) indicating successful fabrication of very low resistive ohmic Ni contacts on $6 \mathrm{H}-\mathrm{SiC}$ in the range of about $20 \Omega$.

with as deposited $\mathrm{Ni}$ contacts or $\mathrm{Ag}$ back-contacts of comparable size. In order to estimate the ohmic serial resistor of the particular heterojunctions of $\mathrm{SiC}$ based systems, the contact resistances as well as the intrinsic resistance of the semiconducting substrate were analyzed similar to the other MASS diode systems. The I-V characteristics of all three different types of back-contacts are summarized in figure 6.25 . 
Ag back-contacts (colored green) exhibit very high resistances of about $10^{8} \Omega$ likely caused by the formation of Schottky barriers at the interface of the $\mathrm{SiC}$ substrates despite their low resistivity of just $0.05-0.1 \Omega \mathrm{cm}$. As a result, these kind of contacts interfere significantly with the original I-V characteristic of the heterojunction and cannot be entirely corrected by the SMU using a remote measurement setup as the internal resistance of the SMU is in the same order of magnitude.

On the other hand, the evaporation of pure Ni back contacts onto the back-side of the SiC-substrates, as described in section 2.3.2, lead to a decrease in resistivity of about three orders of magnitude and an I-V characteristic (black squares) closer to ohmic conduction. The subsequent annealing process eventually results in another significant increase of conductivity of approximately four orders of magnitude and an almost perfect linear I-V characteristic (blue circles) indicating successful fabrication of very low resistive ohmic Ni contacts on $6 \mathrm{H}-\mathrm{SiC}$. The resulting resistances are in the range of about $20 \Omega$ and primarily based on the resistivity of the $\mathrm{SiC}$ substrates as well as the distance of the contact pads. Therefore, the contact resistances are negligible.

\subsection{1 t-BN/SiC heterostructures}

Starting with samples containing t-BN thin-films, another type of MASS heterojunction is formed by $\mathrm{Au} / \mathrm{Cr} / \mathrm{t}-\mathrm{BN} / \mathrm{SiC}$ heterostructures. The corresponding image of a typical I-V characteristic at room temperature is illustrated in figure 6.26a. The heterojunctions show a pronounced diode characteristic extending over a bias voltage range of $\pm 20 \mathrm{~V}$ but exhibit a rectifying behavior of just one order of magnitude. Based on similar structural properties (see section 5.3.6) compared to p-type silicon based samples as well as on the successful application of the model to two different MASS heterostructures in the previous section, the FPID-model was also applied to the I-V-characteristic of the $\mathrm{t}-\mathrm{BN} / \mathrm{SiC}$ heterojunctions.

Comparing the recorded dataset (black circles) with the numerically simulated FPID-model (red solid line), an almost perfect fit at forward-bias can be observed. However, there is a progressively increasing discrepancy between the datasets and the simulated I-V curves at reverse-bias exceeding $-4 \mathrm{~V}$. Based on the results of the sections above, this bias-region of MASS heterojunctions is primarily dominated by leakage currents of the diode and therefore affected by the composition and presence of defects.

Therefore, the focal point of the analysis is shifted towards the impact of structural properties regarding the $\mathrm{SiC}$-surface as well as the interface region between $\mathrm{SiC}$ and the grown thin-film onto the electrical properties of the diode component of the ECD in order to correct the flaws of the conduction model in this bias-region.

According to the TEM-analysis in section 5.3.6, the growth of the t-BN thin-film is not direct with respect to the substrate surface due to the formation of an amorphous interlayer of about $5-7 \mathrm{~nm}$ thickness. This interlayer is primarily caused by 

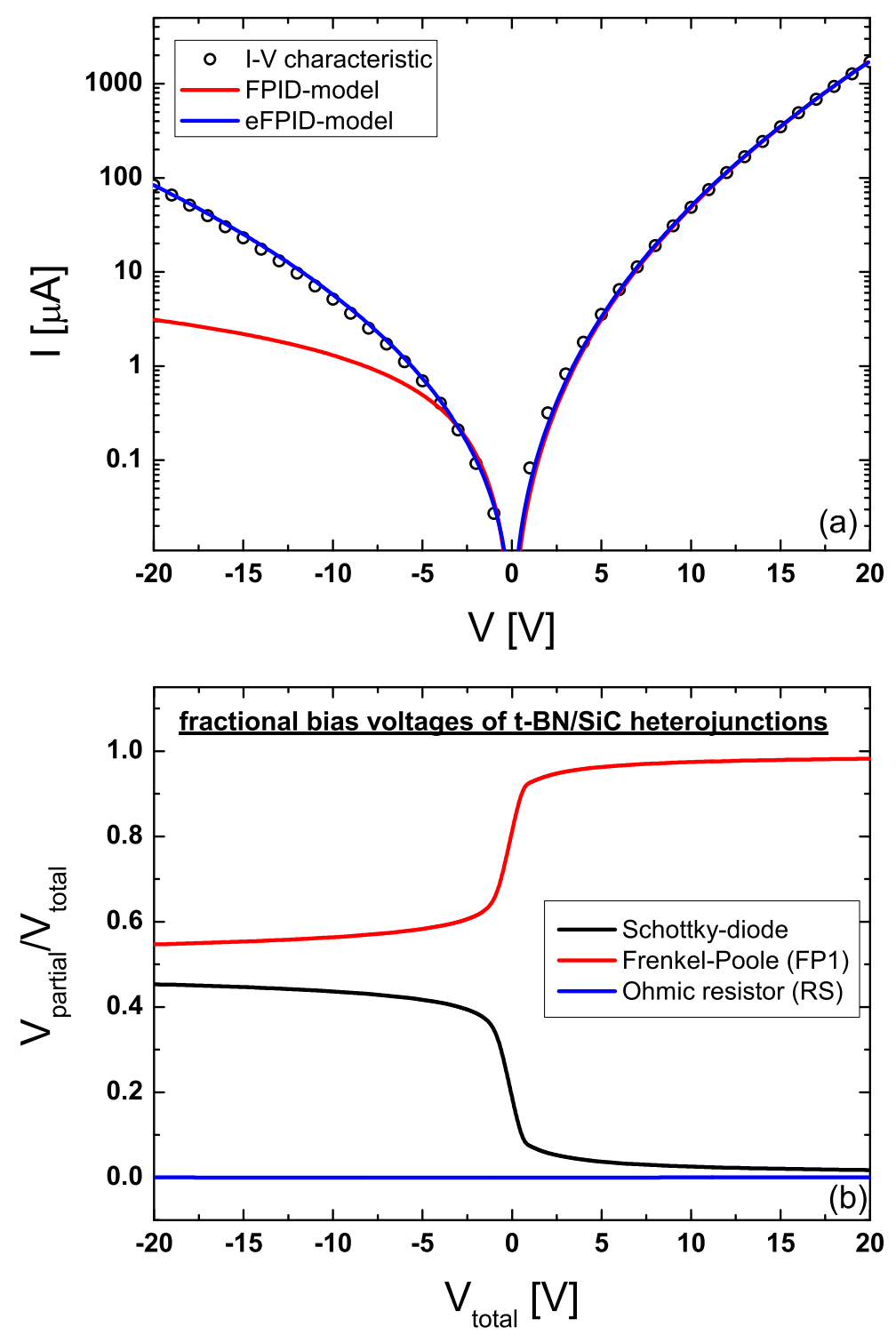

Figure 6.26: (a) I-V characteristic of a typical $\mathrm{Au} / \mathrm{Cr} / \mathrm{t}-\mathrm{BN} / \mathrm{SiC}$ heterojunction at $300 \mathrm{~K}$ (black circles). The numerical simulation of the FPID-model (red solid line) is in accordance with the recorded data in forward-bias but deviates from the measured characteristic at reverse-bias voltages exceeding $-4 \mathrm{~V}$. The discrepancy can be compensated by an additional voltage dependent FrenkelPoole like parasitic resistor resulting in a perfect fit of the numerical simulation (blue solid line) of this extended FPID-model (eFPID model). The corresponding equivalent circuit diagram is shown in figure 6.27. (b) Corresponding fractional bias voltage characteristics based on the numerical simulation of the eFPID-model. 
the sputter-cleaning process using Ar-ions prior to the actual thin-film-growth. In addition, the performed SRIM-calculations indicate implantations of B- and N-ions into the first few nanometers of the amorphous $\mathrm{SiC}$ surface during the deposition process. As a result, the amorphous interlayer exhibits a large number of defect states as well as additional free-charge carriers or even additional doping centers inside an already highly conductive $\mathrm{SiC}$ material.

Therefore, the presence of an additional serial resistor accompanied by a significant voltage drop can be ruled out. Instead, the assumption of increased leakage currents

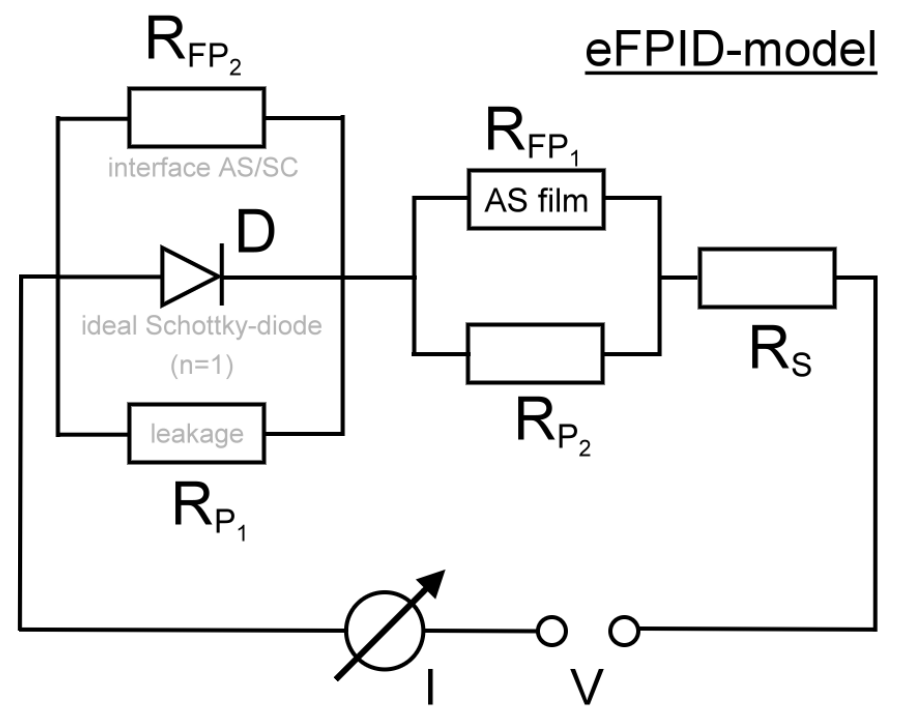

Figure 6.27: Equivalent circuit diagram of the extended Frenkel-Poole and ideal diode model (eFPID-model): The serial arrangement of an ideal Schottky diode, a Frenkel-Poole like resistor $R_{\mathrm{FP} 1}$ and an ohmic resistor $R_{\mathrm{S}}$ is identical to the FPID-model (figure 6.10). In order to emulate the increased and exponentially increasing leakage currents in several MASS heterostructures, an additional voltage dependent Frenkel-Poole like parasitic resistor $R_{\mathrm{FP} 2}$ has been added to the model based on the amorphous character and the high defect density of the interlayer according to TEM-analyses. Furthermore, the original parasitic resistor $R_{\mathrm{P} 1}$ is still maintained in order to represent the ohmic leakage currents inside the depletion region of the crystalline substrate.

of the diode is more reasonable and represented in the corresponding equivalent circuit diagram (see figure 6.27) by an additional leakage resistor arranged parallel to the diode.

Based on the amorphous structure of this region and a high defect density accompanied by the shape of the exponential characteristic of the heterojunction at reverse-bias (see figure 6.26) similar to a thermally activated conduction mechanism, the leakage of the diode is approximated by another voltage dependent resistor $R_{\mathrm{FP} 2}$ with Frenkel-Poole like conduction according to equation 6.1. Due to the lack of information regarding the precise composition and defect density inside the interface region, the parameter ranges cannot be limited by preliminary analyses. However, the thickness of this amorphous interlayer can directly be obtained from the corresponding TEM-analyses of t-BN/SiC heterostuctures in section 5.3.6. 
Furthermore, the original parasitic resistor $R_{\mathrm{P} 1}$ is still maintained in order to represent the ohmic leakage currents inside the depletion region of the crystalline substrate. In this context, the effect of thick amorphous $\mathrm{SiC}$ layers covering some parts of the original wafer surface according to the TEM analysis in sections 5.3.6 and 5.3 .7 can also not be determined as the efforts for an analysis of the whole contact areas, e.g. using TEM-measurements, of each investigated sample are way out of proportion.

Focusing on the numerical simulation of this extended Frenkel-Poole and ideal diode model (eFPID-model), it is in very good agreement with the measured I-V characteristic as illustrated by the blue solid line in figure 6.26a. Comparing the parameters of the retained components of the whole device, all parameters of the diode and the voltage dependent Frenkel-Poole resistor $R_{\mathrm{FP} 1}$, the dimension of the parasitic ohmic leakage resistor $R_{\mathrm{P} 1}$ as well as the ohmic serial resistor $R_{\mathrm{S}}$ have only been slightly adjusted.

Again, the corresponding fractional bias voltages, displayed in figure 6.26 $\mathrm{b}$, provide additional information about the voltage drop across the individual components of the equivalent circuit diagram in figure 6.27 as well as about the resulting impact onto the I-V characteristic of the whole device at different bias voltages.

In the forward-bias region, the exponential increase of the I-V characteristic is almost entirely dominated by a Frenkel-Poole type resistor. Thus, in this region the major voltage drop $(\approx 90-95 \%)$ is across the t-BN film accompanied by a slight contribution from the Schottky diode component at $V \leq 5 \mathrm{~V}$. In addition, the ohmic serial resistor is almost negligible compared to the t-BN thin-film due to the low resistivity of the annealed Ni contacts as well as the highly doped SiC substrate (a few Ohm each) and will only dominate at much higher electrical field intensities. At reverse-bias, the percentage of voltage drop $(\approx 40-45 \%)$ across the diode components is significantly lower compared to MASS diodes based on ZnO as well as p-type Si and is primarily caused by the increased leakage currents of the Schottky diode. As a result, the highly resistive t-BN thin-film provides also a significant contribution of about 55-60 \% at reverse-bias voltages. In this context, the voltage drop across both components will most likely reach saturation limits around $50 \%$ for much higher applied electrical fields.

Therefore, the $\mathrm{I}-\mathrm{V}$ characteristic of a $\mathrm{Au} / \mathrm{Cr} / \mathrm{t}-\mathrm{BN} / \mathrm{SiC}$ heterojunction is governed by the one-sided p-n junction and the corresponding leakage currents at reverse-bias as well as by Frenkel-Poole conduction of the disordered thin-film at forward-bias. The transition between these two regions of the diode is rather abrupt around zerobias and similar to the ta-C/p-Si system (see e.g. figure 6.17).

Finally, temperature dependent I-V measurements in the range of $20 \mathrm{~K}-300 \mathrm{~K}$ were performed in order to verify the validity of the eFPID-model. The I-V characteristics of a typical $\mathrm{Au} / \mathrm{Cr} / \mathrm{t}-\mathrm{BN} / \mathrm{SiC}$ heterojunction (colored circles) as well as the corresponding numerical simulation (solid lines of the same colors) are shown in figure 6.28 $\mathrm{a}$. All recorded I-V characteristics exhibit a pronounced rectifying characteristic, even down to temperatures of just $20 \mathrm{~K}$. Below a temperature of $60 \mathrm{~K}$, 

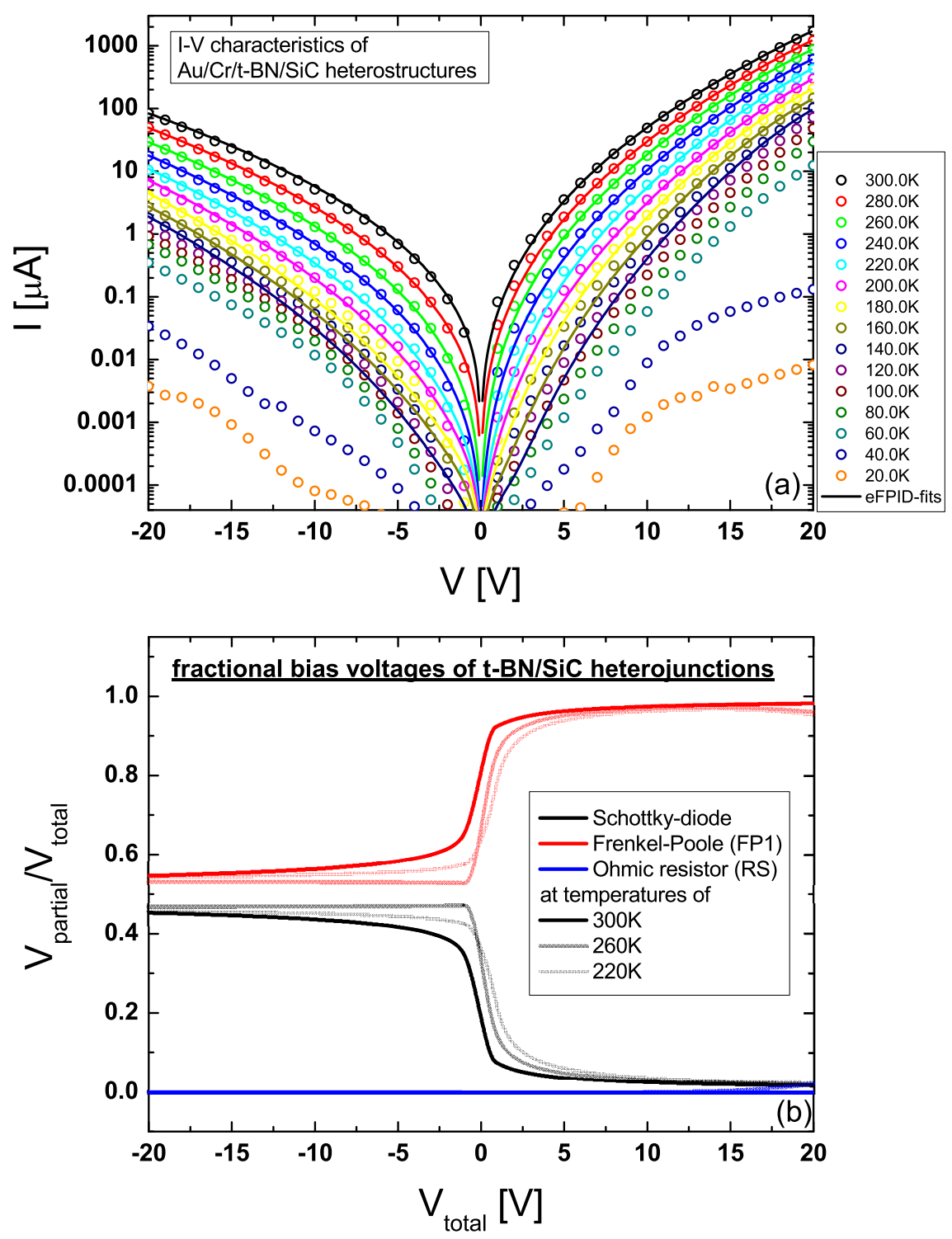

Figure 6.28: (a) Temperature dependent I-V measurements of a typical $\mathrm{Au} / \mathrm{Cr} / \mathrm{t}-\mathrm{BN} / \mathrm{SiC}$ heterojunction (colored circles). All recorded I-V characteristics (colored circles) exhibit a pronounced rectifying characteristic. The numerical simulations of the eFPID-model (colored solid lines) are in very good agreement with the recorded datasets down to a temperature of about $220 \mathrm{~K}-200 \mathrm{~K}$. At lower temperatures, the simulations start to deviate progressively from the recorded datasets with decreasing temperature due to the arising residual conductivity of the t-BN thin-film.

(b) Comparison of fractional bias voltages at different temperatures of $300 \mathrm{~K}, 260 \mathrm{~K}$ and $220 \mathrm{~K}$ based on the corresponding numerical simulations of the eFPID-model. 
the resistances dominating the characteristics especially at high electrical fields such as $R_{\mathrm{S}}$ as well as $R_{\mathrm{P} 1}$ and $R_{\mathrm{FP} 2}$ increase considerably by several orders of magnitude up to values of about $1 \cdot 10^{9} \Omega$. Similar to the other systems, this behavior is most likely caused by the semiconducting substrate.

Focusing on the numerical simulations of the eFPID-model, the calculated datasets are in very good agreement with the recorded I-V measurements. In particular, the reverse-bias direction of the I-V characteristics is suitably described by the eFPIDmodel at reverse-bias with respect to the temperature dependence in addition to the appropriate simulation of the voltage dependent leakage currents of each temperature step. Therefore, the predicted presence of a second Frenkel-Poole like resistor dominating at reverse-bias is in fact a reasonable assumption. Similar to the systems

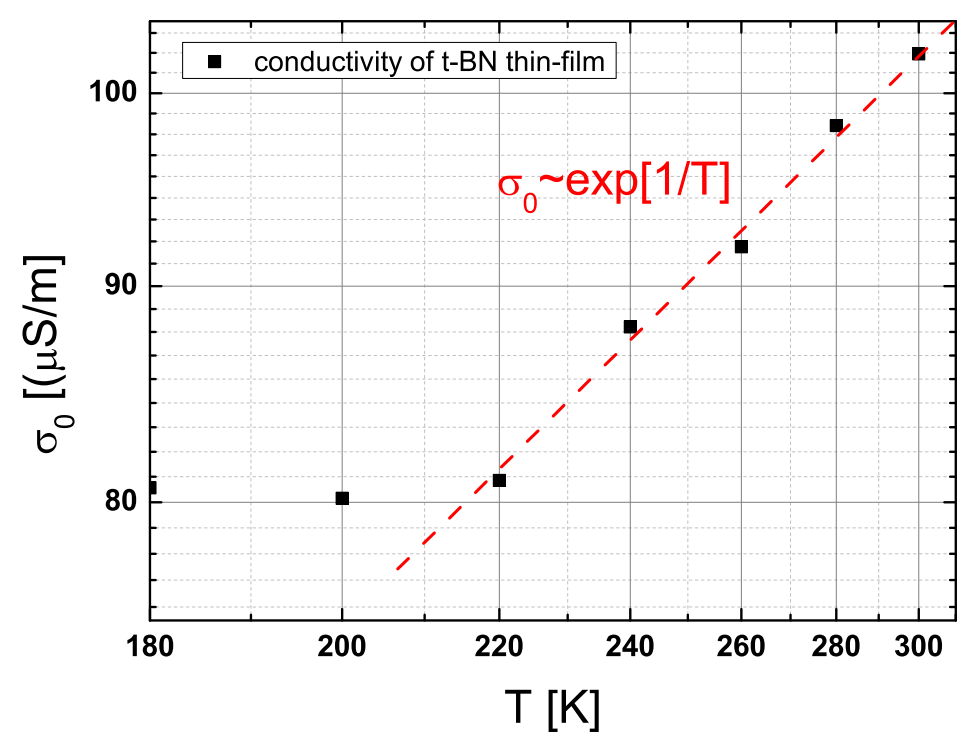

Figure 6.29: Arrhenius-plot regarding the conductivity $\sigma_{0}$ of the t-BN thin-films inside t-BN/SiC heterostructures extracted from the numerical simulation data in figure 6.28. Again, $\sigma_{0}$ exhibits a temperature dependence proportional to $\exp (1 / \mathrm{T})$ but the corresponding values are about one order of magnitude lower compared to the t-BN reference samples.

investigated before, the corresponding conductivity of the t-BN thin-film, extracted from the numerical simulation data, also exhibits a temperature dependence proportional to $\exp (1 / \mathrm{T})$ as shown in figure 6.29 but the corresponding values are about one order of magnitude lower compared to the t-BN reference samples coincidentally matching the difference of original substrate resistances.

At lower temperatures between $200 \mathrm{~K}$ and $180 \mathrm{~K}$ the simulations start to deviate from the recorded datasets in a low forward-bias range of $0 \mathrm{~V}-5 \mathrm{~V}$ underestimating the actual current of the samples. Furthermore, the discrepancies between the simulation and the recorded I-V characteristics become more pronounced at lower temperatures. This discrepancies primarily increases at lower electric fields which 
are dominated by the Frenkel-Poole like conduction mechanism of the grown thinfilm according to the fractional bias voltages in figure6.26 b. Again, these results are in accordance with the emergence of residual conductivity inside the t-BN thin-films as shown in section 6.1.1 based on t-BN reference samples.

In addition, the comparison of fractional bias voltage characteristics at different temperatures of $300 \mathrm{~K}, 260 \mathrm{~K}$ and $220 \mathrm{~K}$ is shown in figure $6.28 \mathrm{~b}$ and provides further information about the effect of different temperatures on the individual components inside the MASS diode structure.

At these temperatures, the characteristics of all components are much alike and differ only by a few percent and shape around zero-bias. In more detail, the shift towards a complete voltage drop across the t-BN thin-film becomes more smooth in forward-bias direction. In addition, a slight contribution of a few percent from the ohmic serial resistor arises at high electric fields at around $260 \mathrm{~K}$. In contrast, the shift towards a voltage drop across the diode components becomes more abrupt at lower temperatures and the saturation limits of the voltage drops across both components are almost reached at low electric field.

However, around $260 \mathrm{~K}$ the characteristic differs from the predicted trend of temperature dependence at reverse-bias and intersects the fractional bias characteristic of $220 \mathrm{~K}$ around zero-bias. This is most likely caused by the rather complex interference of four individual components in this bias-region all exhibiting different or even opposing temperature dependencies.

In principle, the observed shift in fractional bias voltages is still caused by the different temperature dependencies between the Frenkel-Poole dominated t-BN thin-film and the depletion region inside the semiconducting substrate including all present leakage effects. 


\subsection{2 ta-C/SiC heterostructures}
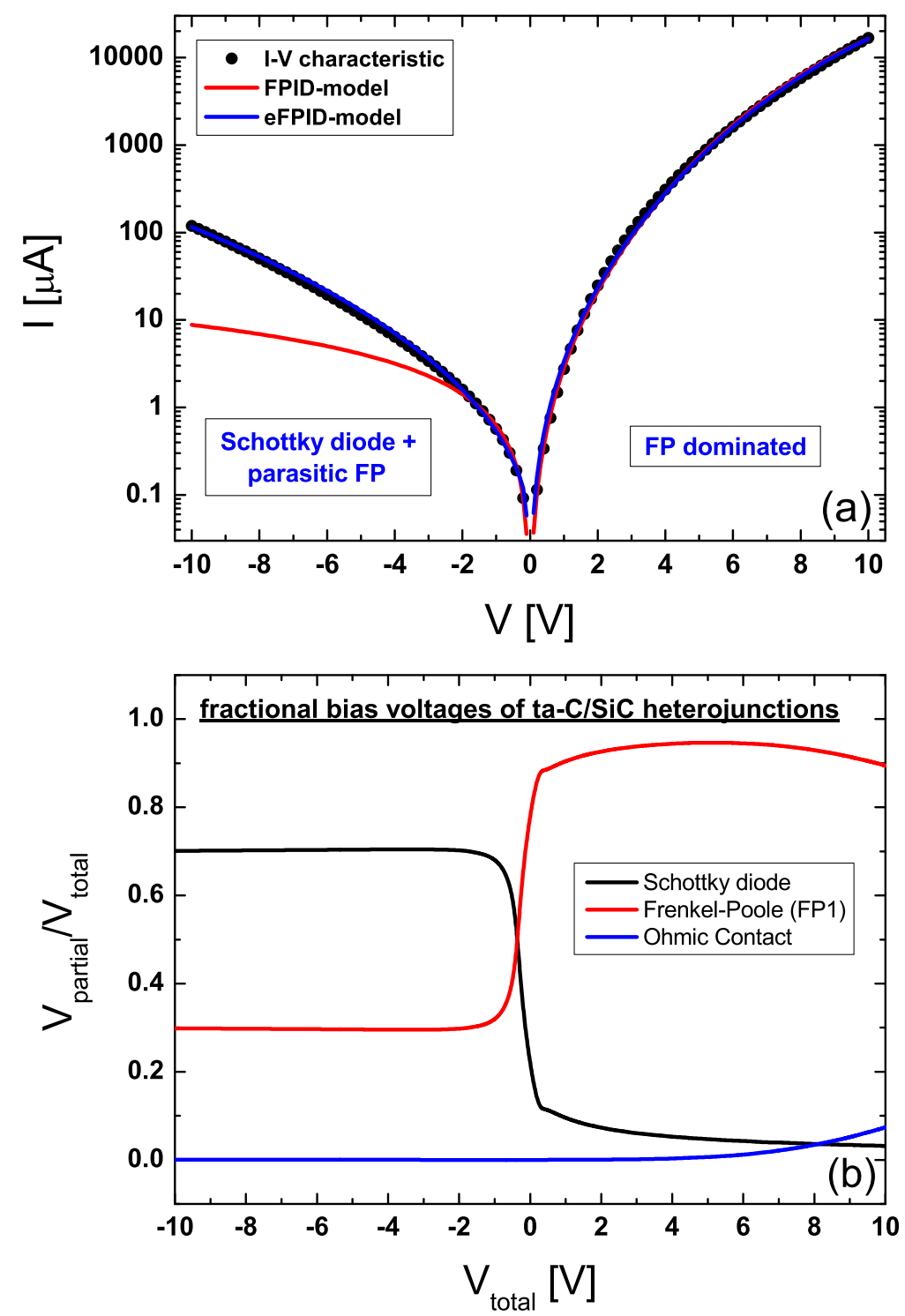

Figure 6.30: (a) I-V characteristic of a typical $\mathrm{Au} / \mathrm{Cr} / \mathrm{ta}-\mathrm{C} / \mathrm{SiC}$ heterojunction at room temperature. The numerical simulation of the eFPID-model (blue solid line) is in very good agreement with the recorded datasets (black circles). The discrepancy of the FPID-model (red solid line) at reverse-bias is very similar to the t-BN based system and also progressively increasing with the applied bias voltage. (b) Corresponding fractional bias voltage characteristics based on the numerical simulation of the eFPID-model. 
Based on the TEM-analysis in section 5.3.7 the ta-C/SiC system exhibits very similar layered structure compared to t-BN/SiC heterostructures. Therefore, the eFPID-model according to figure 6.27 was also applied to the I-V characteristics of typical $\mathrm{Au} / \mathrm{Cr} / \mathrm{ta}-\mathrm{C} / \mathrm{SiC}$ heterojunctions at room temperature as shown in figure 6.30 a. The rectifying effect of the MASS diode has a value of about two orders of magnitude and is therefore considerably larger than in $\mathrm{SiC}$ based heterojunctions containing a t-BN thin-film. Furthermore, the leakage current of the diode as well as the conductivity at forward-bias are significantly higher in ta-C based samples compared to the t-BN/SiC system. In detail, the conductivity at reversebias is increased by a factor of about 20 while the conductivity of the ta-C/SiC heterostructures at forward-bias is even approximately 200 times higher compared to the $\mathrm{t}-\mathrm{BN} / \mathrm{SiC}$ system.

Nevertheless, the numerical simulation of the eFPID-model (blue solid line) is still in very good agreement with the recorded datasets (black circles) in figure 6.30a. In addition, a numerical simulation of the FPID-model was also performed for comparison reasons and is illustrated by the red solid line in figure 6.30 a. The discrepancy at reverse-bias is very similar to the t-BN based system and also progressively increasing with the applied bias voltage. Except for the additional voltage dependent leakage resistor $R_{\mathrm{FP} 2}$, the corresponding parameters of the remaining individual components regarding the equivalent circuits of the FPID- as well as the eFPID-model are almost identical, similar to the $\mathrm{t}-\mathrm{BN} / \mathrm{SiC}$ system, supporting the assumption of similar structural and electrical properties. The interaction of the individual components is analyzed on the basis of the corresponding fractional bias voltage characteristics shown in figure 6.30

At forward-bias, the major voltage drop takes place across the Frenkel-Poole like resistor $R_{\mathrm{FP} 1}(\approx 90 \%)$ accompanied by slight contributions from the diode components $(\leq 10 \%)$. In this context, the small bulge around zero-bias is mainly caused by the complex interference of four different resistors with significant contribution percentages. However, based on the higher conductivity of ta-C compared to t-BN and the resulting more pronounced exponential increase of the corresponding I-V characteristic, a slight contribution of the ohmic serial resistor $R_{\mathrm{S}}$ arises at around $6 \mathrm{~V}$ and increases progressively at higher electric fields at the expense of the FrenkelPoole like resistor. In addition, as the Schottky diodes of both $\mathrm{SiC}$ based systems are supposedly almost identical due to their structural properties obtained from TEM-analyses and the identically processed $\mathrm{Au} / \mathrm{Cr}$ top-contacts, the higher conductivity of ta-C compared to t-BN also leads to a reduced contribution from the Frenkel-Poole resistor of the grown thin-film at reverse-bias. As a result, the voltage drop across the diode components is increased leading to enhanced currents with respect to the total bias voltages of the two systems. Therefore, the reverse-bias region of the MASS diode is dominated by the diode components but still exhibits a significant contribution from the ta-C thin-film and the saturation limits of $\approx 70 \%$ (diode) and $\approx 30 \%\left(R_{\mathrm{FP} 1}\right)$ are already reached at low bias voltages around $2 \mathrm{~V}$. In addition, the leakage current of the whole device can also be affected by slight 
variations in the electrical properties of the diode components themselves. In this context, the implantation of carbon-ions instead of boron and nitrogen into the sputtered SiC-surface might result in a slight variation of stoichiometry or differences in doping levels compared to t-BN/SiC heterostructures and therefore may also lead to changes regarding the leakage current of the diode. In addition, slight differences in sputter-yields and straggling effects between the particular types of deposited ions according to sections 5.3.6 and 5.3.7 as well as the arrangement of the implanted ions inside the amorphous SiC-matrix might also influence the electrical properties of the diode component. Due to the lack of suitable analysis techniques, these effects could not be examined during this thesis.

The validity of the eFPID-model and the individual components within were once again verified using additional temperature dependent measurements in the range of $20 \mathrm{~K}-300 \mathrm{~K}$. The corresponding I-V characteristics of a typical $\mathrm{Au} / \mathrm{Cr} / \mathrm{ta}-\mathrm{C} / \mathrm{SiC}$ MASS diode are shown in figure 6.31a. The numerical simulations (colored solid lines) are in very good agreement with the recorded datasets (colored circles) down to a temperature of about $160 \mathrm{~K}$. Again, the corresponding conductivity of the ta- $\mathrm{C}$ thin-film inside the ta-C/SiC heterostructures, extracted from the numerical simulation data, also exhibits a temperature dependence proportional to $\exp (1 / \mathrm{T})$ as shown in figure 6.32. However, compared to the t-BN/SiC system, the corresponding values are even lower and differ by about two orders of magnitude from those of ta-C reference samples.

At lower temperatures, the simulations start to differ from the I-V measurements especially in the forward-bias region dominated by Frenkel-Poole conduction of the ta-C thin-film. Similar to all MASS diodes investigated before, the actual current values of the recorded I-V characteristics are underestimated by the calculated datasets of the eFPID-model. In contrast, the reverse-bias region of the heterojunction can be approximated in an appropriate way except for very low electric fields. As the arising discrepancies as well as the corresponding temperature ranges are consistent with the results obtained from ta- $\mathrm{C}$ reference sample and from ta- $\mathrm{C} / \mathrm{Si}$ heterojunctions, the failure of the eFPID-model is once more primarily related to the residual conductivity of the grown ta-C thin-film.

Finally, the comparison of fractional bias voltage characteristics at different temperatures of $300 \mathrm{~K}, 260 \mathrm{~K}$ and $220 \mathrm{~K}$ is shown in figure 6.31 b and provides additional information about the temperature dependencies of the individual components of the MASS heterojunction. In general, the contributions of the diode components become more dominant with decreasing temperatures exclusively at the expense of the Frenkel-Poole like resistor of the ta-C thin-film. As a result, the voltage dependent transition area completely shifts from the reverse- towards the forward-bias region of the characteristic. Therefore, the fractional bias percentages become almost static at reverse-bias and successively converge towards a constant ratio of fractional bias voltages at low temperatures with corresponding saturation limits at high electric fields of about $75 \%$ and $25 \%$, respectively. In forward-bias direction, on the other hand, the increase of contributions related to the grown thin-film be- 

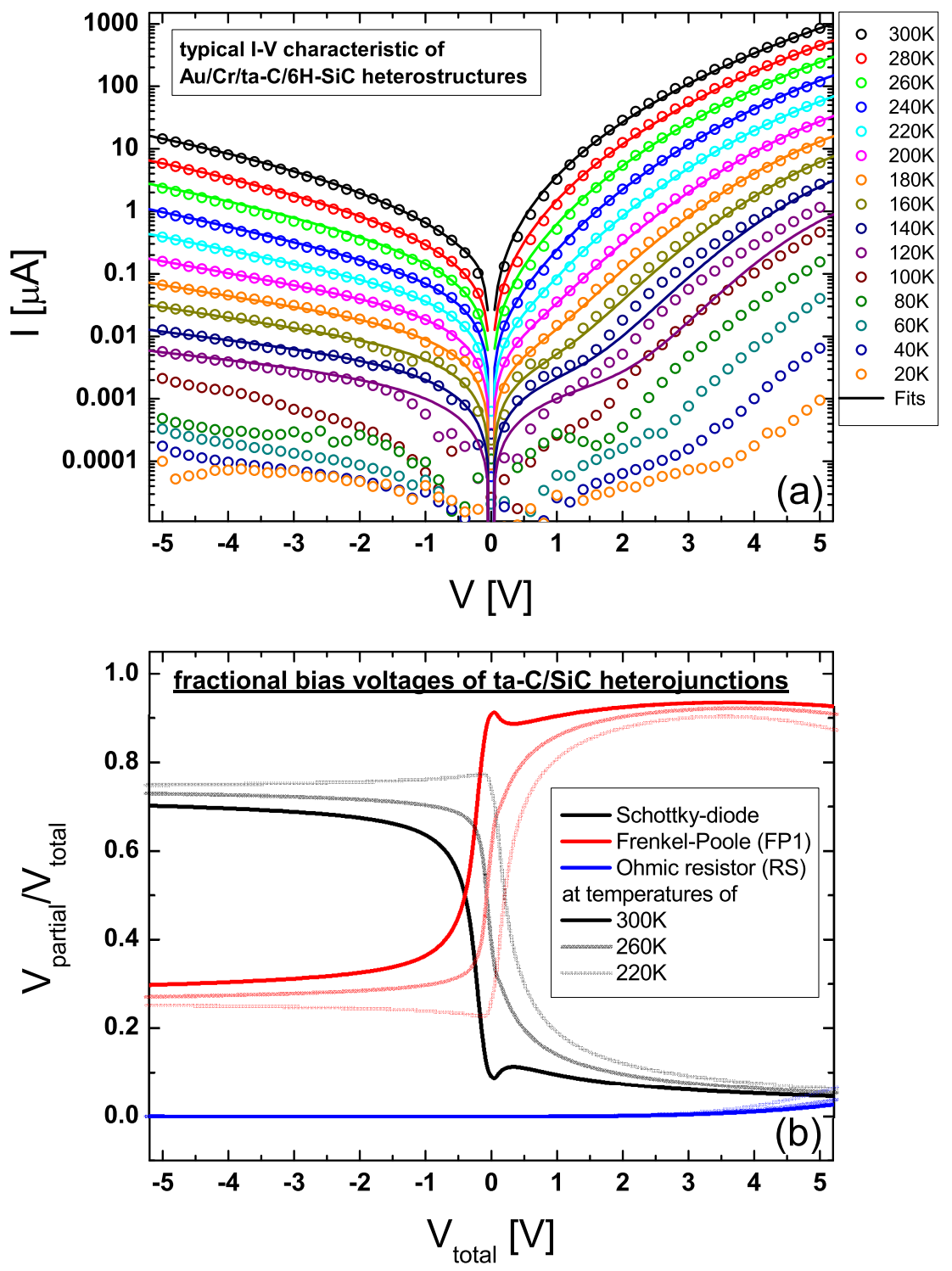

Figure 6.31: (a) Temperature dependent I-V measurements of a typical $\mathrm{Au} / \mathrm{Cr} / \mathrm{ta}-\mathrm{C} / \mathrm{SiC}$ heterojunction. The numerical simulations of the eFPID-model (colored solid lines) are in very good agreement with the recorded datasets down to a temperature of about $160 \mathrm{~K}$. At lower temperatures, the simulations start to differ from the I-V measurements especially in the forward-bias region dominated by Frenkel-Poole like conduction due to the residual conductivity of the ta-C thin-film. (b) Comparison of fractional bias voltage characteristics at different temperatures of $300 \mathrm{~K}, 260 \mathrm{~K}$ and $220 \mathrm{~K}$ based on the corresponding numerical simulations of the eFPID-model. 


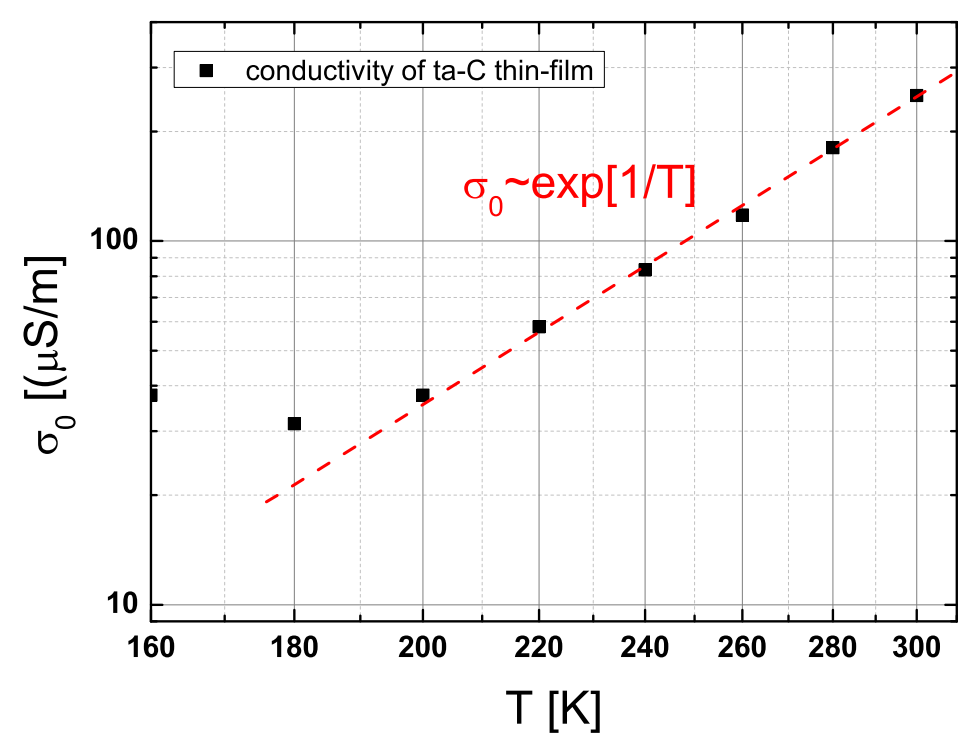

Figure 6.32: Arrhenius-plot regarding the conductivity $\sigma_{0}$ of the ta- $\mathrm{C}$ thin-films inside ta- $\mathrm{C} / \mathrm{SiC}$ heterostructures extracted from the numerical simulation data in figure 6.31. The temperature dependence is proportional to $\exp (1 / \mathrm{T})$ and the values differ by about two orders of magnitude from those of ta-C reference samples.

come less distinct at lower temperatures and a contribution from the ohmic serial resistor additionally arises at higher electric fields indicating a significant voltage drop across the $\mathrm{SiC}$ substrate. 


\subsubsection{The effect of $\mathrm{Cu}$ contaminants}

Similar to the reference samples analyzed in section 6.1.3, also a few SiC based heterostructures are partially contaminated by a small amount of $\mathrm{Cu}$. The resulting I-V characteristic as well as the corresponding fractional bias voltages are exemplarily shown on the basis of a $\mathrm{Au} / \mathrm{Cr} / \mathrm{t}-\mathrm{BN} / \mathrm{SiC}$ heterojunction in figure 6.33. The most significant difference compared to $\mathrm{I}-\mathrm{V}$ characteristics of typical t-BN/SiC samples (see figure 6.26) are the significantly elevated current-levels at low and mid-level electric fields in forward-bias indicating a considerable increase in conductivity of the t-BN thin-film. The reverse-bias direction, in contrast, exhibits a very similar voltage dependent conductivity 5 . In addition, the numerical simulation of the extended FPID-model (gray solid line) is still in very good agreement with the recorded dataset. In this context, based on the previous results of $\mathrm{Cu}$-contaminated reference samples, only the curvature parameter $\zeta$ as well as the conductivity of the t-BN thin-film have been varied significantly whereas the average barrier height was kept constant. Furthermore, the remaining parameters such as parasitic and serial ohmic resistors as well as diode parameters are very similar to typical t-BN/SiC heterojunctions.

The effects of $\mathrm{Cu}$-contaminants can additionally be visualized using the corresponding fractional bias voltage characteristics in figure 6.33b.

In comparison with typical heterojunctions of the t-BN/SiC system (see figure 6.26), the voltage drop across the t-BN thin-film is considerably reduced due to the increased conductivity of the t-BN thin-film. As a result, contributions from the diode components at reverse-bias as well as the ohmic serial resistor at forward-bias become significantly more dominant. At reverse-bias, almost the entire voltage drop takes place across the diode components accompanied by a slight and progressively increasing contribution from the t-BN thin-film. In this context, the ratio is even more pronounced than inside typical ta-C/SiC heterojunctions (see figure 6.30) also indicating a high conductive thin-film. At forward-bias, the contribution from the t-BN thin-film is limited to lower electric fields and superseded by the ohmic serialresistor at higher field intensities.

Nevertheless, in summary, the eFPID-model is still valid for $\mathrm{Cu}$-contaminated heterojunctions and the shifts of fractional bias voltages can entirely be linked to a electrical modification of the grown thin-film.

\footnotetext{
${ }^{5}$ Note: This sample exhibits a significantly lower film thickness as well as larger contact areas compared to the heterojunction shown in figures 6.26 and 6.28
} 

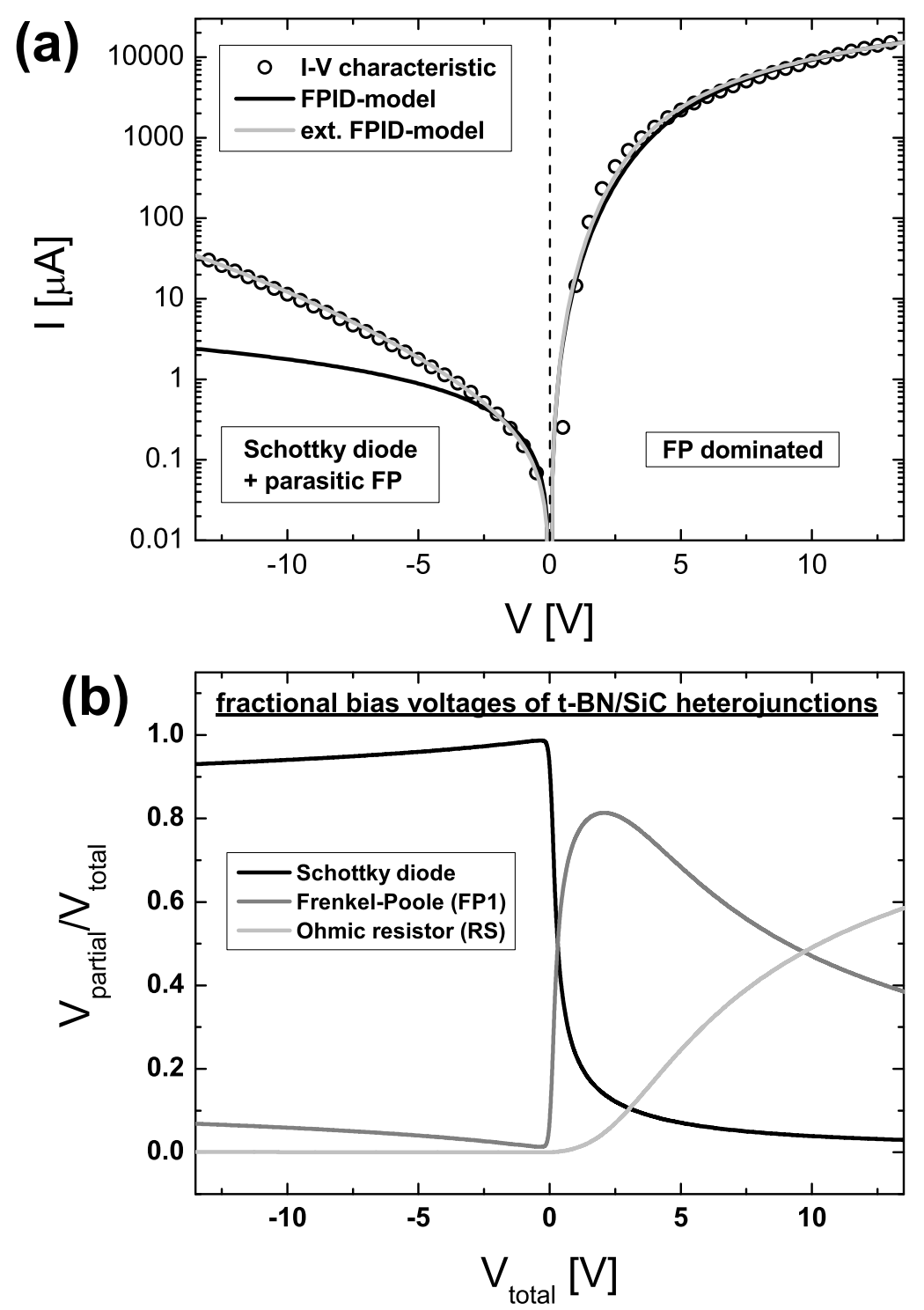

Figure 6.33: (a) $\mathrm{I}-\mathrm{V}$ characteristic of a typical $\mathrm{Au} / \mathrm{Cr} / \mathrm{t}-\mathrm{BN} / \mathrm{SiC}$ heterojunction containing $\mathrm{Cu}-$ contaminants (open circles, taken from reference [150]). (a) The most significant difference compared to typical t-BN/SiC samples (see figure 6.26) are the significantly elevated current-levels at low and mid-level electric fields in forward-bias indicating a considerable increase in conductivity of the t-BN thin-film. Again, the numerical simulation (blue solid line) of the eFPID-model is in very good agreement with the recorded data while the calculated characteristic of the FPID-model deviates from the recorded measurement at higher reverse-bias voltages. (b) Corresponding fractional bias voltage characteristics based on the numerical simulation of the eFPID-model. The contributions from the diode components at reverse-bias as well as the ohmic serial resistor at forward-bias become significantly more dominant while the contribution from the t-BN thin-film is limited to lower electric fields at forward-bias. 


\subsection{Additional MASS heterostructures}

In another step, additional heterojunctions based on the ta- $\mathrm{C} / \mathrm{ZnO}$ as well as the t-BN/p-Si system have been analyzed during this work. Due to the absence of suitable temperature dependent I-V measurements in remote configuration, both MASS diode systems were only investigated with respect to their conformity with the developed conduction models.

\subsection{1 t-BN/p-Si heterostructures}

Starting with the t-BN/p-Si system, all samples were investigated using a local measurement setup and large area Ag back-contacts. However, they exhibit a pronounced rectifying characteristic of about two orders of magnitude and an identical polarity compared to the ta-C/p-Si system. The I-V characteristic of a typical $\mathrm{Au} / \mathrm{Cr} / \mathrm{t}-\mathrm{BN} / \mathrm{p}-\mathrm{Si}$ heterostructure (black circles) is shown in figure 6.34. In comparison to ta-C based samples on the same substrates the t-BN/p-Si system exhibits relatively large leakage currents. Based on the TEM-analysis in section 5.3.4, the structural properties are very similar to ta-C/p-Si heterostructures in terms of the layered structure as well as the implantation effects into the silicon substrate. Nevertheless, they are also comparable to t-BN/SiC based MASS diodes with respect to the sequence of layers. Therefore, numerical simulations of the FPID- (red solid line) as well as the eFPID-model (blue solid line) were performed. Both of them are also illustrated in figure 6.34.

The eFPID-model is in good agreement with the recorded data whereas the application of the FPID-model leads to the well-known discrepancies at reverse-bias of the heterojunction. Furthermore, the serial resistor of both conduction models is in the range of just a few $\mathrm{k} \Omega$ and therefore very similar to the ta-C/p-Si system indicating a low resistive Ag back-contact.

The corresponding fractional bias voltages of the eFPID-simulation are also shown in figure 6.34. The forward-bias region is primarily dominated by Frenkel-Poole like conduction of the t-BN thin-film accompanied by a slight contribution from the diode components low electric fields. In addition, there is also a progressively increasing voltage drop across the ohmic serial resistor at higher electric fields. In contrast, the reverse-bias region of the heterojunction is dominated by the diode components with a maximum of nearly $100 \%$ voltage drop around zero-bias. However, due to the high leakage current of the diode and a high resistivity of the t-BN thin-film, an additional contribution of the FP-resistor related to the t-BN thin-film arises at reverse-bias and increases progressively at increasing field-intensity.

Comparing the electrical properties of the two p-Si based heterojunctions, both of them can be well described by the simpler FPID-model in forward-bias direction and at low electric-fields at reverse-bias. The increased leakage current of the Schottky diode, on the other hand, can only be explained by the eFPID-model despite similar structural properties overall. Regarding the growth process of the thin-films, described in sections 5.3.4 and 5.3.5, this effect can be caused by the different com- 

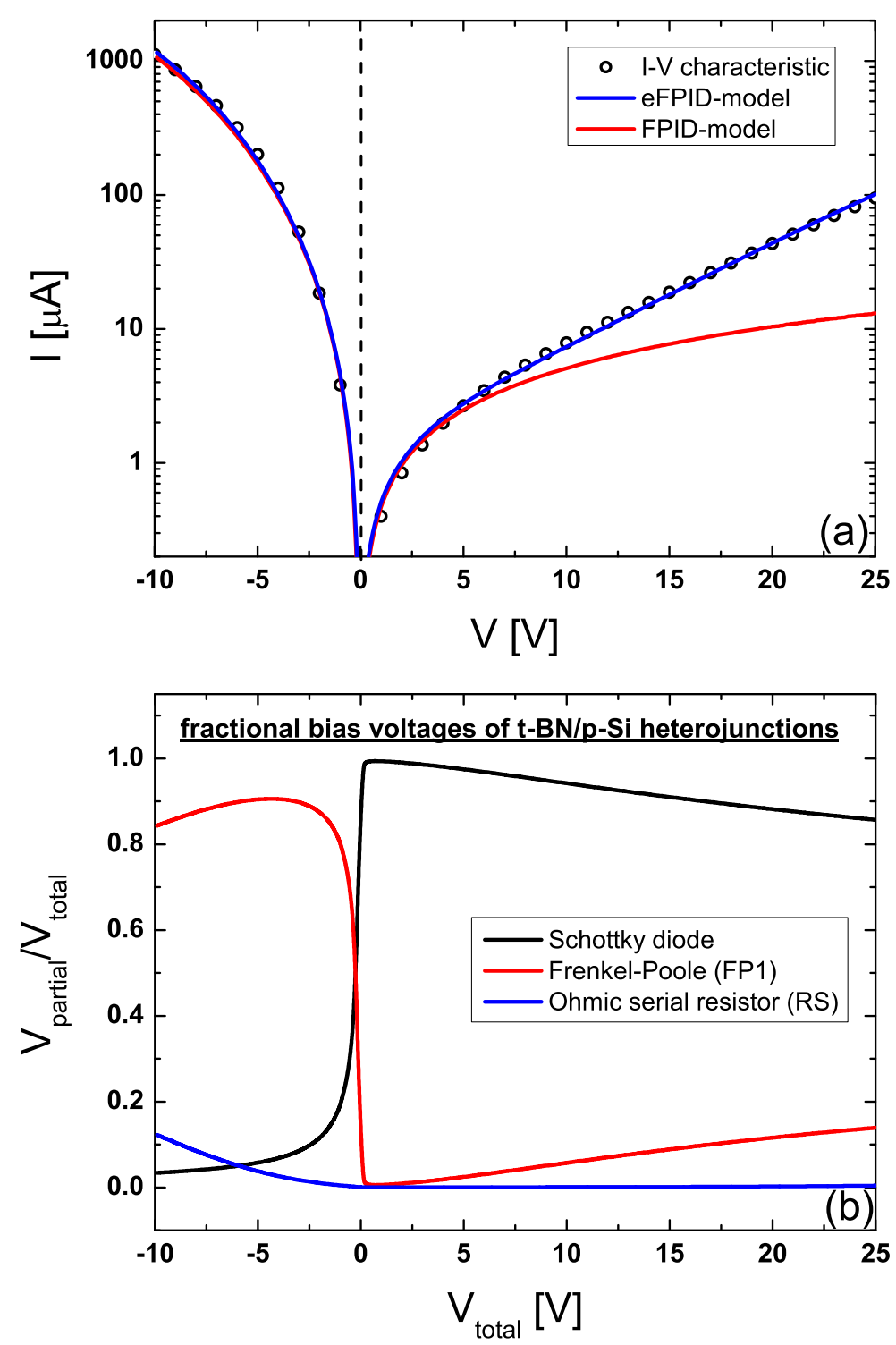

Figure 6.34: (a) I-V measurement (black circles) of a typical Au/Cr/t-BN/p-Si heterostructure at room temperature. The numerical simulations of the eFPID-model (blue solid line) are in good agreement with the recorded data whereas the application of the FPID-model (red solid line) leads to the well-known discrepancies at reverse-bias of the heterojunction. This is in accordance with the structural properties obtained from the TEM-analysis of the t-BN/p-Si system.

(b) Corresponding fractional bias voltage characteristics of the individual components with respect to the eFPID-model simulation.

position of the intermixing layer as it contains B- and N-ion instead of C. As boron and nitrogen (especially boron) are frequently used in the doping process of silicon devices based on ion-implantation, the implantation of these ions might lead to the formation of a significant number of additional acceptor- or donor-states inside the 
silicon surface which would result in a weakening of the Schottky barrier stability. Carbon-ions, in contrast, will likely form some kind of $\mathrm{Si}-\mathrm{C}$ compound or only slightly increase the conductivity of the surface layer. In addition, the turbostratic and therefore "semi- or micro-crystalline" character of the t-BN thin-film compared to the amorphous structure of ta-C might also result in a different interface formation with respect to the sputtered amorphous surface layer of the semiconducting substrate interfering with the original barrier stability.

\subsection{2 ta-C/ZnO heterostructures}

Another MASS diode system is formed by $\mathrm{Au} / \mathrm{Cr} / \mathrm{ta}-\mathrm{C} / \mathrm{ZnO}$ heterostructures. The $\mathrm{I}-\mathrm{V}$ characteristic of a typical sample is shown in figure 6.34. In comparison to the t-BN/ZnO system in section 6.2, the rectifying characteristic is less pronounced accompanied by significantly increased leakage currents as well as increased overall conductivity. However, numerical simulations of the FPID-conduction model are in good agreement with the recorded datasets. An appropriate simulation is illustrated by the blue solid line in figure 6.35. Due to the lack of an appropriate TEM-analysis of the ta- $\mathrm{C} / \mathrm{ZnO}$ system, the underlying structural properties can only be derived from the results of the $\mathrm{t}-\mathrm{BN} / \mathrm{ZnO}$ system. Therefore, a direct growth of the ta-C thin-film without the formation of an additional amorphous interlayer is predicted. Furthermore, an intermixing layer will likely be formed by the implanted carbonions into the $\mathrm{ZnO}$ crystal during deposition.

Based on the results of the previous sections, ta-C exhibits a much lower resistivity compared to t-BN. Therefore, the voltage drop across a similar diode component of a heterojunction containing $\mathrm{ZnO}$ would be increased compared to the $\mathrm{t}-\mathrm{BN} / \mathrm{ZnO}$ system leading to a corresponding increase in current. In addition, the leakage of the diode might also be affected by the different composition of the intermixing layer due to the incorporation of carbon instead of boron and nitrogen.

In this context, the voltage drop across the individual components of the heterojunction is analyzed in more detail on the basis of the corresponding fractional bias voltage characteristics shown in figure 6.35. The forward-bias region is primarily dominated by the Frenkel-Poole resistor but also exhibits a progressively increasing contribution from the serial resistor caused by the $\mathrm{ZnO}$ substrate. Compared to t-BN based samples, the contribution of the serial resistor $R_{\mathrm{S}}$ is much stronger and even present at higher voltages at reverse-bias.

For $V \leq 2.5 \mathrm{~V}$ at reverse-bias, the characteristic is also primarily dominated by the FP-resistor. However, the voltage drop progressively shifts towards the diode components with increasing field intensity. At high electric fields in the reversebias region, almost the entire voltage drop $(\geq 80 \%)$ takes place across the diode components accompanied by a slight contribution from the FP-resistor of the thinfilm $(\approx 20 \%)$. Even though the corresponding electric fields are significantly lower compared to the t-BN/ZnO system, the fractional bias voltage characteristic of ta$\mathrm{C} / \mathrm{ZnO}$ samples at reverse-bias is almost identical in a qualitative way indicating a 

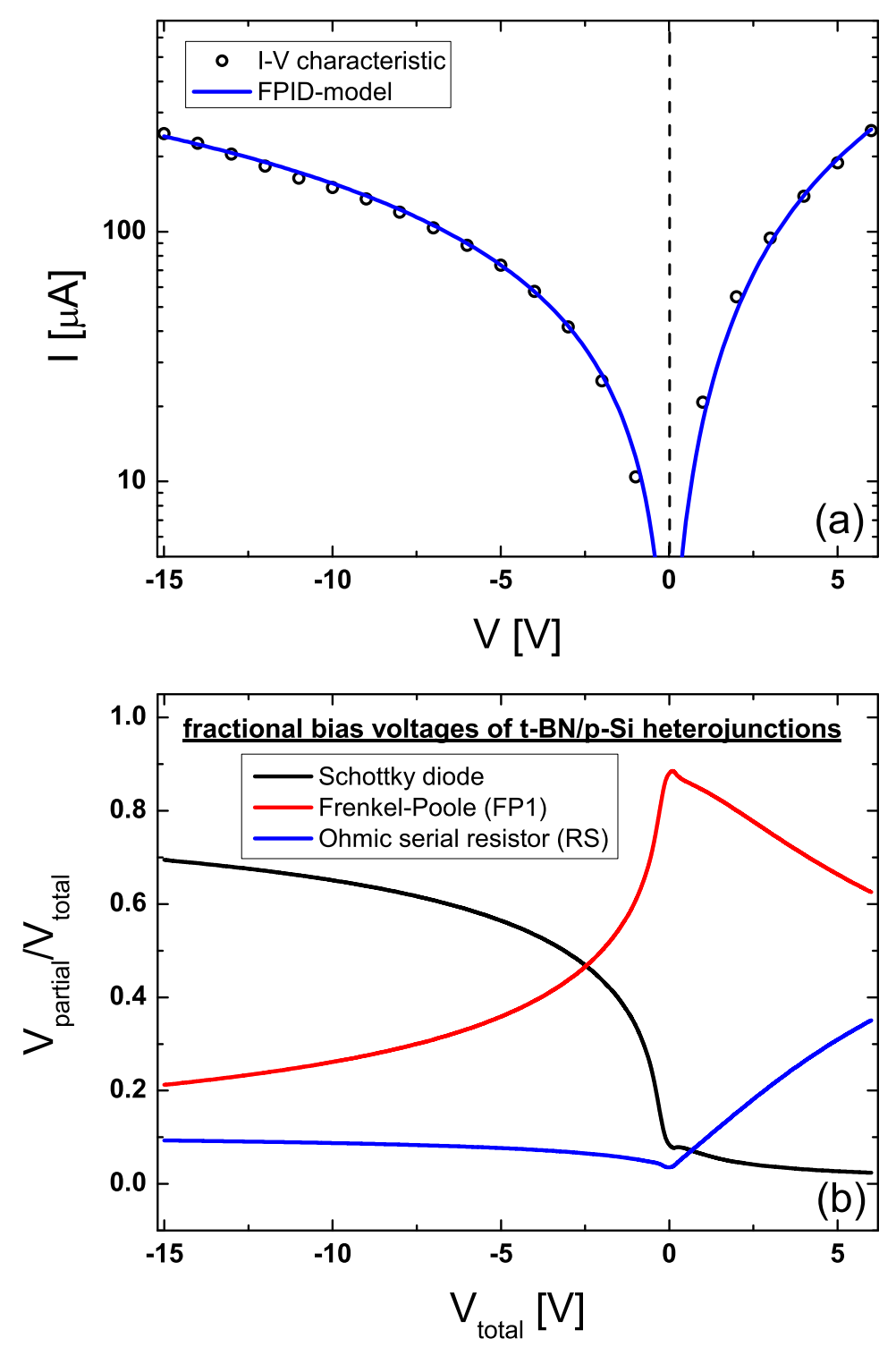

Figure 6.35: (a) I-V characteristic of a typical $\mathrm{Au} / \mathrm{Cr} / \mathrm{ta}-\mathrm{C} / \mathrm{ZnO}$ heterostructure at room temperature. Compared to $\mathrm{t}-\mathrm{BN} / \mathrm{ZnO}$ samples in section 6.2, the rectifying characteristic is less pronounced accompanied by significantly increased leakage currents as well as overall conductivity. However, numerical simulations of the FPID-conduction model are in good agreement with the recorded datasets. (b) Corresponding fractional bias voltage characteristics of the individual components with respect to the FPID-model simulation.

very similar conduction mechanism. Moreover, the distinct shift of the fractional bias voltage percentage towards the serial resistor at forward-bias is primarily caused by the large resistivity of the $\mathrm{ZnO}$ substrate interfering with the highly voltage dependent conductivity of the ta-C thin-film. As a result, the recorded datasets are still in accordance with the predicted characteristic of the FPID-model. 


\subsection{Summary}

As predicted on the basis of the structural characterization results obtained from TEM-analyses in chapter 5, the electrical properties of turbostratic boron nitride (t-BN) as well as tetrahedral amorphous carbon (ta-C) thin-films are dominated by a Frenkel-Poole like conduction mechanism in all grown heterostructures of this work.

Due to the imperfections of the original one-dimensional Frenkel-Poole conduction model, described in section 3.3, the Frenkel-Poole model (FP-model) of this work was improved in order to cover additional conduction mechanisms dominating at low electric fields and temperatures as well as possibly present leakage currents of the thin-film matrices. As a result, the symmetric characteristics of the thin-films can be well described by a parallel arrangement of a Frenkel-Poole like resistor $R_{\mathrm{FP}}$, dominating the characteristic at higher temperatures and electric fields, and a parasitic resistor $R_{\mathrm{P}}$ which may also include contributions from variable range hopping and/or tunneling at lower electric fields and temperatures. Therefore, $R_{\mathrm{P}}$ has been approximated by an ohmic resistor in first-order.

Furthermore, the static parameter of conductivity $\sigma_{0}$ has been replaced by a temperature dependent one and a curvature factor $1 / \zeta$ has been added accompanying the dielectric constant $\epsilon_{r}$ of the original FP-formula in order to sustain the dielectric properties while describing the I-V characteristics more accurately.

The corresponding numerical simulations of this uniform FP-model, shown in figure 6.3 and based on equation 6.1, are in very good agreement with the recorded datasets down to temperatures of $240 \mathrm{~K}$ for t-BN and $200 \mathrm{~K}$ for ta-C reference samples on metallic n-type silicon. Below those temperatures, the simulations start to differ from the actual measurements as the dominating conduction mechanism inside the thin-films changes accompanied by a progressive deviation of temperature dependencies from the predicted trend regarding the free parameters of the model as illustrated by corresponding Arrhenius-plots of each system in figures 6.4 and 6.6. Finally, the temperature dependencies vanish almost completely below temperatures of about $125 \mathrm{~K}$ for t-BN and $100 \mathrm{~K}$ for ta-C (less pronounced) leading to the formation of "plateau"-regions of residual conductivity of the thin-films similar to a tunneling conduction mechanism described in section 3.7 .

Based on these results, the electrical properties of MASS heterostructures on real semiconducting substrates were investigated. All of these systems exhibit pronounced rectifying characteristics and possess high stability with respect to electric field intensities across the deposited thin-films in the range of approximately $1 \cdot 10^{7} \mathrm{~V} / \mathrm{cm}$. However, the characteristics also exhibit unusually high turn-on voltages and apparently high ideality factors of 30-200 in terms of the Shockley theory of diode characteristics, described in section 3.5. In this context, the values were obtained by using numerical simulations of a non-ideal diode model (NID-model) in figure 6.13 . 
Due to almost identical structural properties of each type of grown thin-film regardless of composition of the semiconducting substrate, this unusual characteristics could generally be linked to the presence of Frenkel-Poole like conduction mechanisms dominating at forward-bias of all MASS diode systems.

In addition, the rectifying behavior of the heterostructures has been linked to the presence of an one-sided p-n junction with reasonable Schottky barrier heights between the top-metal contacts and the particular semiconducting substrate. This was exemplarily illustrated on the basis of silicon based MASS diodes in section 6.3 as a change of doping type exclusively results in an inversion of polarity regarding the diode characteristic while the remaining parameters such as resistivity, saturation currents and curvature remain almost constant. Moreover, the Schottky barrier height depends on the composition of the evaporated top-contacts and corresponding work-functions with respect to the semiconducting substrates.

Furthermore, preliminary analyses of different back-contact materials on all semiconducting substrates provide additional information about present contact and intrinsic substrate resistances in each MASS diode system as well as possible interferences with the actual characteristic of the heterojunction. Therefore, the recorded dataset of all heterojunctions in this thesis can basically be well described by a serial arrangement of an ideal Schottky diode $\mathrm{D}(n=1)$, a voltage dependent resistor exhibiting Frenkel-Poole like conduction $R_{\mathrm{FP} 1}$ and an additional ohmic resistor $R_{\mathrm{S}}$ covering present contact and substrate resistances. In this context, the corresponding leakage currents of the diode component have been linked to the structural properties of the interface region between crystalline semiconductor surface and the grown thin-films obtained from TEM-analyses in chapter 5. As a result, the leakage of the diode is approximated by a parasitic ohmic resistor $R_{\mathrm{P} 1}$ in order to represent the ohmic leakage currents inside the depletion region of the crystalline substrate and an additional Frenkel-Poole like resistor $R_{\mathrm{FP} 2}$ in case of a present amorphous interlayer interfering with the original heterojunction.

The corresponding equivalent circuit diagram of this extended Frenkel-Poole and ideal diode model (eFPID-model) is shown in figure 6.36. The resulting numerical simulations of this general conduction model are in very good agreement with the I-V characteristics of all investigated heterojunctions and were successfully applied to a total of eight different MASS diode heterostructures (six of them shown in this work). In this context, the effect of each part of the heterostructure on the electrical properties of the resulting heterojunction diode characteristic can be analyzed with respect to the applied bias voltage on the basis of fractional bias voltage characteristics of the individual components regarding the eFPID-model.

Furthermore, the validity of the model as well as of the individual components within have been verified by additional temperature dependent measurements and corresponding numerical simulations. Deviations from the predicted characteristics below temperatures in the range of $150 \mathrm{~K}-200 \mathrm{~K}$, depending on the particular heterostructure compositions, are mainly caused by the residual conductivity of the grown t-BN and ta-C thin-films as well as imperfections of the ohmic approxima- 


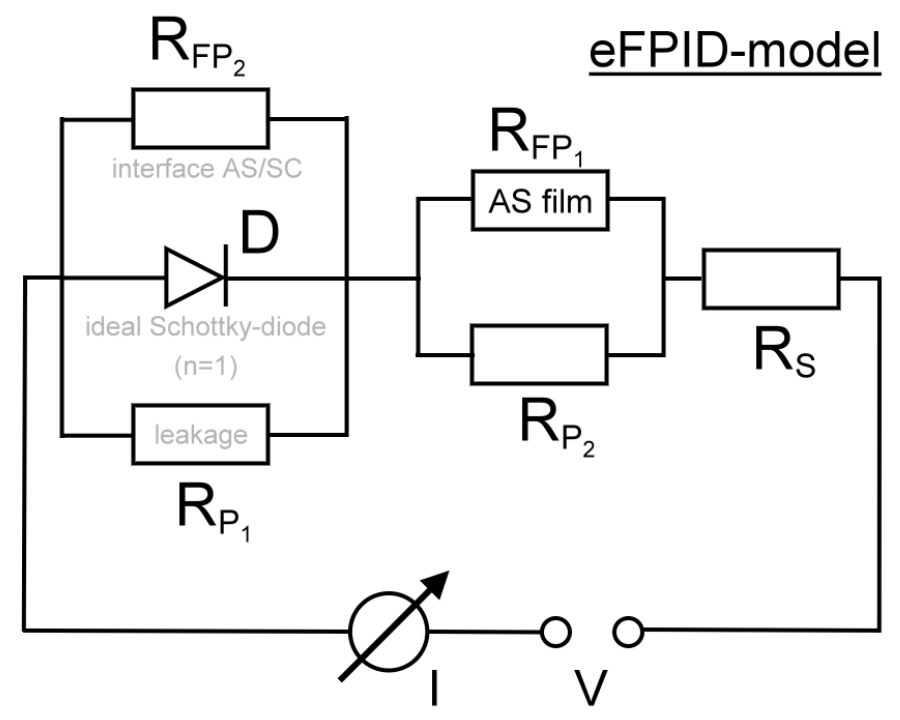

Figure 6.36: Equivalent circuit diagram of the extended Frenkel-Poole and ideal diode model (eFPID-model): A serial arrangement of an ideal Schottky diode $\mathrm{D}(n=1)$, a voltage dependent resistor exhibiting Frenkel-Poole like conduction $R_{\mathrm{FP} 1}$ and an additional ohmic resistor $R_{\mathrm{S}}$ covering present contact and substrate resistances. Based on the structural properties of the interface region between crystalline semiconductor surface and the grown thin-films obtained from TEM-analyses, the leakage of the diode is approximated by a parasitic ohmic resistor $R_{\mathrm{P} 1}$ in order to represent the ohmic leakage currents inside the depletion region of the crystalline substrate and an additional Frenkel-Poole like resistor $R_{\mathrm{FP} 2}$ in case of a present amorphous interlayer interfering with the original heterojunction. The resulting numerical simulations of this general conduction model are in very good agreement with the I-V characteristics of all investigated heterojunctions and were successfully applied to a total of eight different MASS diode heterostructures.

tions regarding the I-V characteristics of leakage and serial resistors. Nevertheless, the consistency of the conduction model was exemplarily illustrated on the basis of Arrhenius-plots regarding the thin-film conductivity $\sigma_{0}$ and resulting temperature dependencies proportional to $\exp (1 / \mathrm{T})$ in various heterojunction systems.

In addition, the conductivity of the grown thin-films is considerably lowered by at least one order of magnitude inside all MASS diode systems compared to the corresponding reference samples on metallic Si. Moreover, a comparison of thin-film conductivity parameters $\sigma_{0}$ of the different heterostructures of this work at room temperature, extracted from corresponding numerical simulations and shown in table 6.3, indicates some kind of correlation between the conductivity of the grown thin-films and the original composition as well as resistivity of the original semiconducting substrates. This is likely caused by the fabrication process of the MASS heterostructures, in particular, by the growth process of the thin-film using the MSIBD-technique. Based on the results of corresponding TEM-analyses in chapter 5. each heterostructure exhibits a rather thin layer of mixed atoms originating from the deposited thin-film as well as from the substrate surface. Therefore, the formation of intermixing layers accompanied by dynamic sputtering effects during the 


\begin{tabular}{|c|c|c|}
\hline $\begin{array}{c}\text { MASS } \\
\text { diode } \\
\text { system }\end{array}$ & $\begin{array}{c}\text { thin-film } \\
\text { conductivity } \\
{[\mathrm{S} / \mathrm{m}]}\end{array}$ & $\begin{array}{c}\text { substrate } \\
\text { resistivity } \\
{[\Omega \mathrm{cm}]}\end{array}$ \\
\hline \hline $\mathrm{t}-\mathrm{BN} / \mathrm{n}^{++}-\mathrm{Si}$ & $0.05-0.2$ & $0.005-0.01$ \\
\hline $\mathrm{t}-\mathrm{BN} / \mathrm{ZnO}$ & 0.00035 & $1000^{*}$ \\
\hline $\mathrm{t}-\mathrm{BN} / \mathrm{SiC}$ & 0.01 & $0.005-0.01$ \\
\hline $\mathrm{ta}-\mathrm{C} / \mathrm{n}^{++}-\mathrm{Si}$ & $1-10$ & $0.005-0.01$ \\
\hline $\mathrm{ta}-\mathrm{C} / \mathrm{p}-\mathrm{Si}$ & 0.1 & 10 \\
\hline $\mathrm{ta}-\mathrm{C} / \mathrm{SiC}$ & 0.025 & $0.005-0.01$ \\
\hline
\end{tabular}

Table 6.3: Comparison of thin-film conductivity parameters $\sigma_{0}$ of the different heterostructures of this work at room temperature. *: estimated value based on I-V characteristics of contact reference measurements.

deposition processes might affect the composition of the grown thin-films due to an incorporation of dopant or substrate atoms from the original substrate. As a result, the conductivity would increase. However, the effect would still be limited by the solubility and related distribution of substrate atoms as well as related doping effects inside the respective t-BN or ta-C matrix. Unfortunately, these assumptions cannot be verified in the absence of an adequate sensitive analysis technique regarding the exact composition on an atomic level and further detailed analyses of thin-film composition are mandatory in order to improved the conduction models in future experiments.

Finally, the effects of unintentional $\mathrm{Cu}$-contaminants on the conduction mechanism of the grown thin-films have also been covered in this thesis. As $\mathrm{Cu}$ contaminants only lead to an increase in conductivity in the range of 30-40 in ta-C as well as in t-BN thin-films while the average barrier height is almost unaffected. Therefore, as shown in sections 6.1.3 and 6.4.3, the conduction models of this work are still valid for small amounts of $\mathrm{Cu}$-contaminants.

In summary, a generalized temperature dependent conduction model has been developed for all grown MASS heterojunction diodes in the scope of thesis considering the structural properties derived from the results of performed TEManalyses of each particular heterostructure as well as the related electrical properties and corresponding temperature dependencies regarding the individual components forming the heterojunction. 


\section{Chapter 7}

\section{Photoresponse of MASS diodes}

In addition to the temperature dependent electrical characterization in DC-mode all MASS diode heterostructures were analyzed with respect to their photoresponse properties as described in section 3.8 using the setups described in detail in section 4.4. As all fabricated heterojunction diodes exhibit a pronounced photosensitivity and a variety of response characteristics when exposed to a light source, the main focus of this chapter is on the localization of its origin as well as on possible correlation to the developed general conduction model.

\subsection{Case studies of photoconductivity measure- ments}

Starting with the case studies of photoconductivity measurements using the simple setup described in section 4.4.1, all types of heterostructures were illuminated for $10 \mathrm{~s}$ with a main spot-size of about $4 \mathrm{~mm}^{2}$. The remainder of the samples was also exposed but at a much lower intensity. Based on the cathodo-luminescence spectrum of the "white" LED (figure 4.11), the maximum photon energy provided by this setup has a value of about $2.76 \mathrm{eV}$.

\subsubsection{Reference samples}

As mentioned in section 3.8, the origin of photoconductivity and response effects is typically located inside the space-charge region of semiconductor-based heterojunctions. Due to the high number of doping states inside the metallic silicon substrates of the reference samples and the resulting absence of a real band-gap, a photoresponse is highly unlikely.

Therefore, the photo-sensitivity of the grown thin-films can be investigated on the basis of ta-C and t-BN reference samples. Typical characteristics of the current as a function of time for constant bias voltages are shown in figure $7.1 \mathrm{a}$ and $\mathrm{b}$ for both types of reference samples. The points of irradiation are marked by the red dashed lines. None of them showed any sign of a photoresponse. 

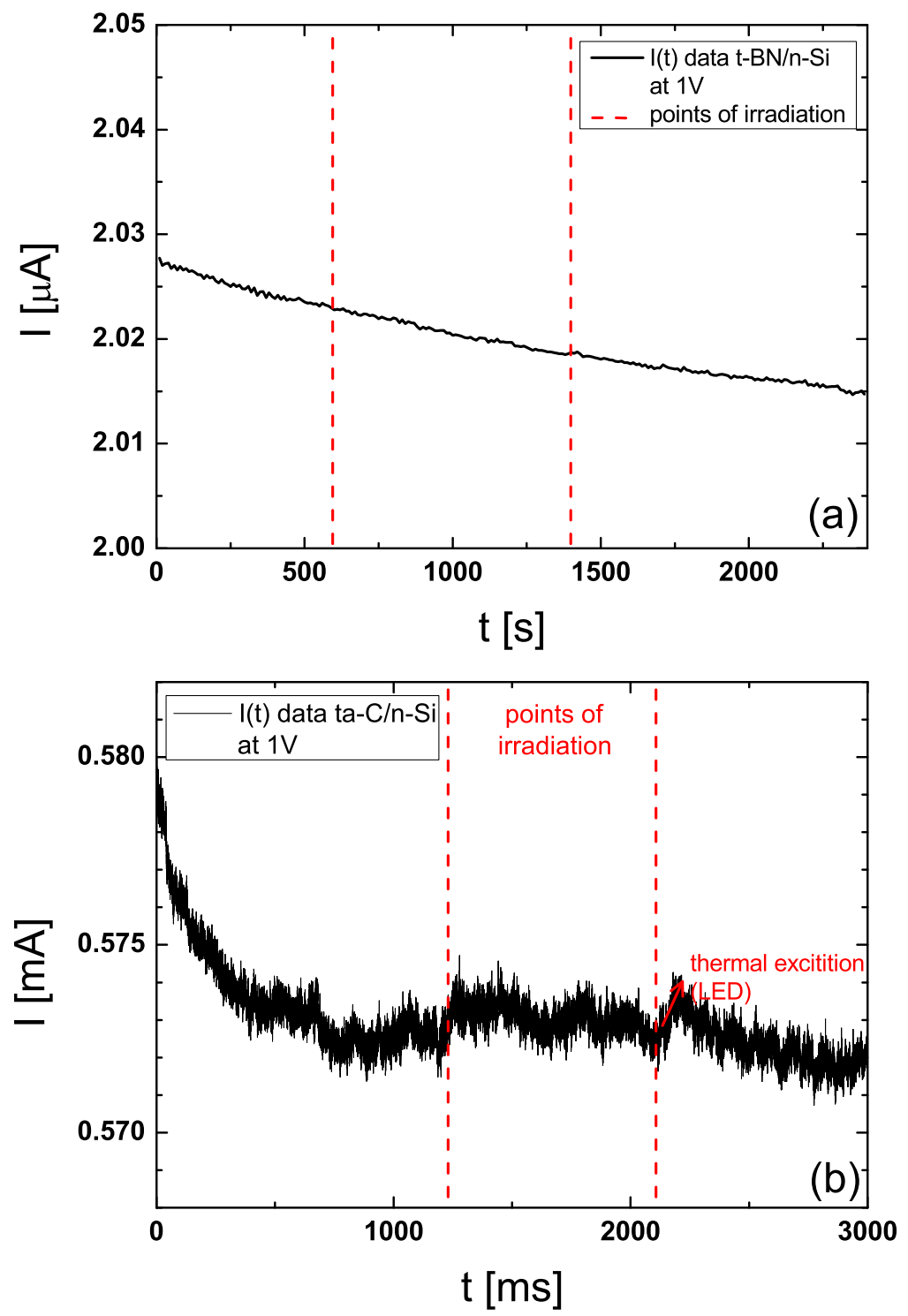

Figure 7.1: Case study photoconductivity measurements of typical t-BN (a) and ta-C (b) reference samples using a "white" LED and the setup described in 4.4.1. The points of irradiation are marked by the red dashed lines. None of them shows any sign of a phoresponse.

In theory, photoconductivity or photoresponse effects are basically caused by the excitation of electrons and holes from the valence band and trapping states into the conduction band of the semiconductor (see figure 3.13 in section 3.8). The absence of any photon induced excitations regarding ta-C as well as t-BN thin-film reference samples on metallic Si is mainly based on high number of defect states in the amorphous and doping centers inside the crystalline semiconductor.

In both materials, excited electrons and holes are almost instantly re-trapped by 
an adjacent defect center or doping atom in the surrounding area. Therefore, the number of additional free charge carriers resulting from possible excitations near the conduction- or valence band of the semiconductor is still within the noise level of the recorded signal and cannot be detected. 


\subsubsection{MASS heterojunctions}

Based on the results of the reference samples, any significant photoresponses of MASS heterojunction systems do not originate from the grown thin-films. Therefore, the particular semiconducting substrate as well as related interface areas of each MASS diode system are the only sources of emerging photo-currents during the irradiation process. Furthermore, all investigated heterojunctions do not show any significant photoresponses at forward-bias, but exhibit a pronounced increase in photo-current during the irradiation-process at applied reverse-bias voltages indicating absorption processes related to the diode components of the heterojunctions. Typical photoconductivity measurements are exemplarily shown in figure 7.2 on the basis of the $\mathrm{t}-\mathrm{BN} / \mathrm{ZnO}$ as well as the $\mathrm{t}-\mathrm{BN} / \mathrm{SiC}$ system. At the beginning of the irradiation process, the conductivity of the sample increases instantly (within the time-resolution of the SMU of about $20 \mathrm{~ms}$ ) followed by an additional but gradual increase in the course of the irradiation. This effect can especially be observed for an irradiation time of $100 \mathrm{~s}$ and most likely originates from thermal excitation due to the proximity of the LED. The corresponding maximum of photo-current has a value of up to $500 \%$ compared to the equilibrium current prior to the exposure. As soon as the LED is switched-off, the current drops abruptly down to one forth of the maximum value followed by a slower decay rate on a much longer time-scale. In this context, the whole decay-process of excited states can be described by a ternary decay-function but only in terms of mathematics. Moreover, such kind of decay is present in all MASS diode systems except of ta-C/p-Si heterostructures.

After exposure, all of them exhibit qualitative similar characteristics and corresponding time constants $\tau_{i}$ of the decay processes of approximately $0.5(3) \mathrm{s}, 15(5) \mathrm{s}$ and a very long decay rate in the range of several hundred seconds. The resulting response times of the particular systems are summarized in table 7.1. In terms of physics, the

\begin{tabular}{|c||c|c|c|}
\hline MASS system & $\tau_{1}$ & $\tau_{2}$ & $\tau_{3}$ \\
\hline $\mathrm{t}-\mathrm{BN} / \mathrm{ZnO}$ & $0.5(3) \mathrm{s}$ & $15(5) \mathrm{s}$ & $170(15) \mathrm{s}$ \\
\hline $\mathrm{t}-\mathrm{BN} / \mathrm{SiC}$ & $0.4(2) \mathrm{s}$ & $15(4) \mathrm{s}$ & $625(42) \mathrm{s}$ \\
\hline $\mathrm{ta}-\mathrm{C} / \mathrm{SiC}$ & $0.45(19) \mathrm{s}$ & $14(3) \mathrm{s}$ & $280(30) \mathrm{s}$ \\
\hline $\mathrm{ta}-\mathrm{C} / \mathrm{p}-\mathrm{Si}$ & $\leq 0.5(3) \mathrm{s}$ & - & $180(25) \mathrm{s}$ \\
\hline
\end{tabular}

Table 7.1: Time constants $\tau_{i}$ obtained from case study photoconductivity measurements.

short decay times can be interpreted as two different relaxation processes of the free charge carriers inside the system. Due to the applied bias, the unoccupied states inside the valence band of the semiconductor can be reoccupied in a very short period of time. Without any irradiation of the sample, the photocurrent between the valence and the conduction band is disrupted almost instantly. Deep level defect states (marked blue in figure 3.13), on the other hand, are reoccupied or depleted at a considerable lower rate based on the limited number inside the interface region and on a larger timescale as a the relaxation of this defect states is mainly based on diffusion of electrons. Furthermore, the longest decay rate of these systems is in the 

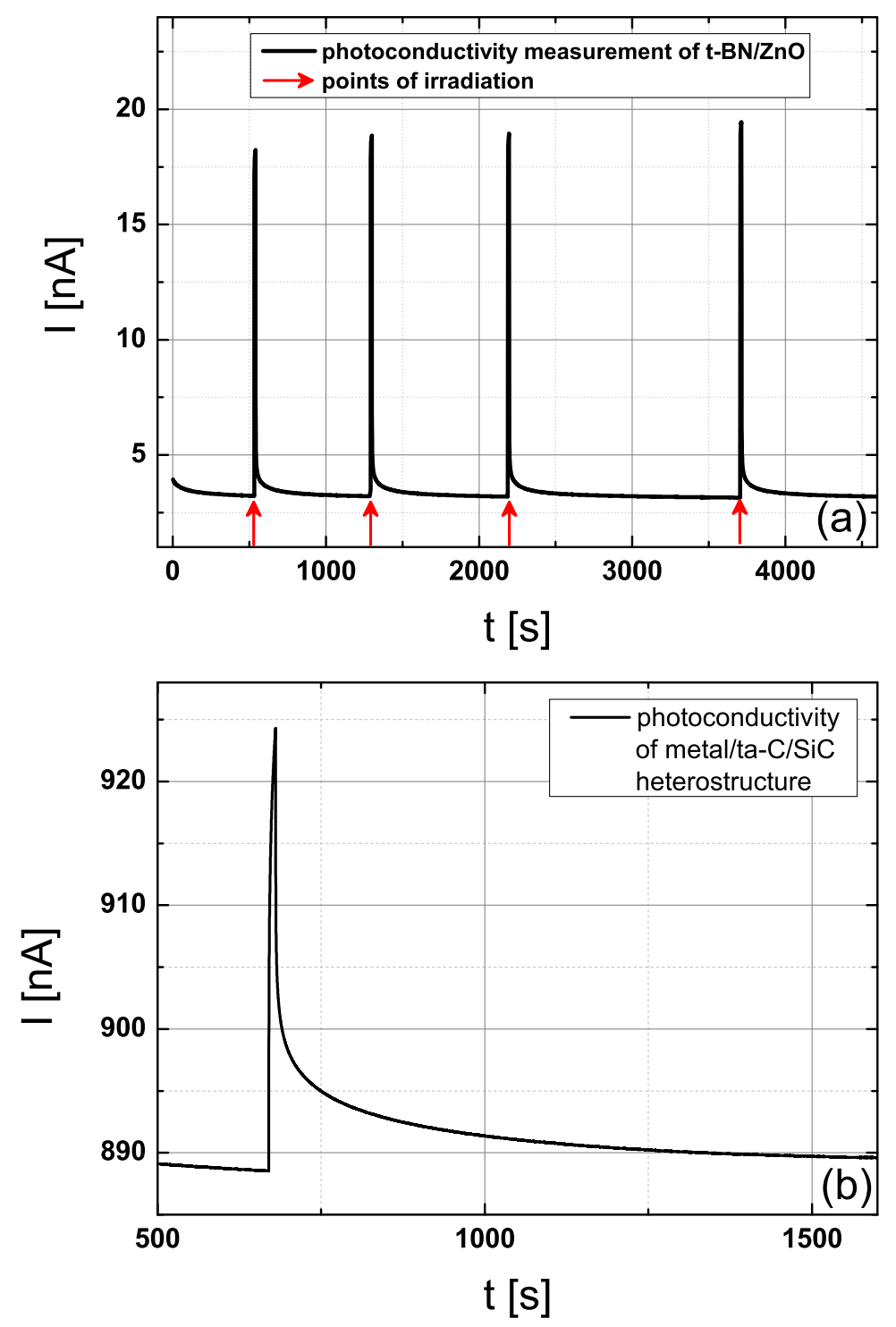

Figure 7.2: Case study photoconductivity measurements of typical MASS diodes exemplarily shown on the basis of the t-BN/ZnO (a) as well as the ta-C/SiC system (b) using a "white" LED and the setup described in 4.4.1. All of them exhibit qualitative similar characteristics and corresponding time constants $\tau_{i}$ regarding the decay processes after exposure of approximately $0.5(3) \mathrm{s}, 15(5) \mathrm{s}$ and a very long decay rate in the range of several hundred seconds. The resulting response times of the particular systems are summarized in table 7.1.

range of several hundred seconds and is most likely also caused by the thermal excitation of the samples as a result of heating provided by the LED during exposure. However, all of these conclusions are just circumstantial and cannot be verified using this simple setup and without any further detailed information in literature. In comparison to the "common" photoconductivity characteristics of MASS diodes in 
this work, the ta-C/p-Si system exhibits a different response. The $\mathrm{I}(\mathrm{t})$ characteristic of a typical sample at a constant reverse bias of $1 \mathrm{~V}$ is shown in figure 7.3 . While

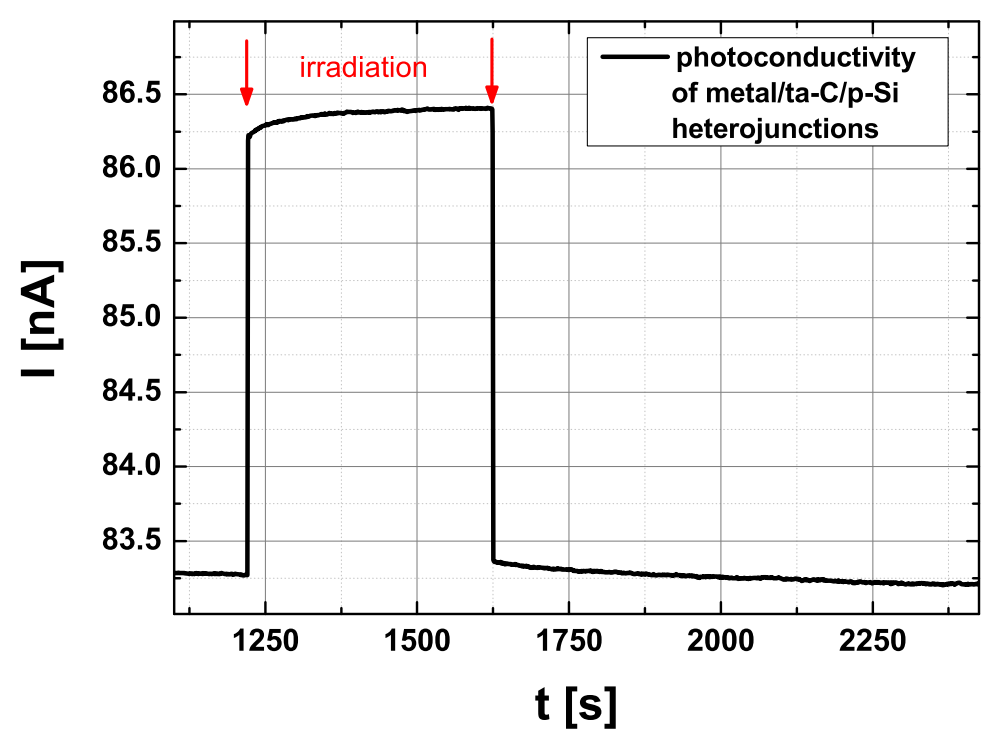

Figure 7.3: Photoconductivity measurements of a ta-C/p-Si heterojunction using a "white" LED and the setup described in 4.4.1. The duration of the irradiation process is marked red and the photoresponse right after irradiation is pronounced. The changes in current are instant in terms of the limited time-resolution of $20 \mathrm{~ms}$ provided by the SMU.

the response of the $\mathrm{I}(\mathrm{t})$ characteristic in the form of an abrupt increase in current is very similar to those of the rest of the MASS heterojunctions during the irradiation, the change in current right after switching-off the LED is instantly in terms of the limited time-resolution of $20 \mathrm{~ms}$ provided by the SMU. In order to analyze these fast photoresponse processes inside the MASS heterojunctions, more precise measurements were performed using the laser assisted time-resolved photoresponse setup described in section 4.4 .2 and are discussed in section 7.2 


\subsection{Time-resolved photoresponse measurements}

In order to investigate the origin of photo-sensitivity of the MASS diode heterostructures and especially the corresponding decay rates of the excited states in more detail, a much higher time resolution in the nanosecond range is required and provided by the pulsed-laser based setup described in section 4.4.2. Based on the case study results in section 17.1, reverse-bias voltages were applied to the investigated samples during the measurements. In addition, due to the high sensitivity of the setup, the photorespose at zero-bias was also analyzed for every sample. Due to the time jitter of the laser, all recorded datasets shown in the following sections are based on a mean value of 512 measurement sweeps. Nevertheless, compared to the case study system, the time-resolved photoresponse measurement setup provides a much more standardized analysis technique in terms of illuminated area, light intensity and time-resolution of the recorded photoresponse signals.

In order to provide enough energy for excitations inside the particular semiconductor substrate, the bang-gap energies of corresponding intrinsic ones are equal to an upper threshold wavelength of the incident laser beam. These values are summarized in table 7.2. Comparing these values to the available laser lines at $266 \mathrm{~nm}$ (equal

\begin{tabular}{|c|c|c|}
\hline SC & band-gap [eV] & wavelength $[\mathbf{n m}]$ \\
\hline $\mathrm{ZnO}$ & 3.37 & 368 \\
\hline $\mathrm{Si}$ & 1.17 & 1060 \\
\hline $6 \mathrm{H}-\mathrm{SiC}$ & 3.0 & 413 \\
\hline
\end{tabular}

Table 7.2: Corresponding wavelengths of semiconductor band-gaps.

to an energy of $4.67 \mathrm{eV}$ ) and $532 \mathrm{~nm}$ (with an energy of $2.33 \mathrm{eV}$ ), the green laser line only provides a sufficient energy for the silicon substrates. However, in order to generate a large number of photon induced free charge carriers inside the $\mathrm{ZnO}$ as well as the $\mathrm{SiC}$ material, the corresponding samples have to be exposed to the UV-laser line at $266 \mathrm{~nm}$ while the green line will likely not trigger any significant photoresponse.

Furthermore, as also mentioned in section 4.4.2, the beam spot is about $1.5-2 \mathrm{~mm}$ in diameter and the area of the evaporated top-contacts are fully illuminated during the measurement. However, a significant percentage $(\approx 25 \%)$ of each light-pulse is blocked by the Au layer of the top-contact (0.9 $\mathrm{mm}$ in diameter). Therefore, the generated charge carriers likely have to travel relatively long distances inside the substrate before being drained by the applied electric field beneath the contact area. Furthermore, additional reflections at the interface regions of the transparent grown thin-films might also contribute to the resulting photo-currents. As a result, an identical illumination of two different MASS diode systems cannot be guaranteed. However, the results of one particular system are reproducible. 


\subsection{1 $\mathrm{Au} / \mathrm{Cr} /$ ta-C/p-Si heterostructures}

Starting with the time-resolved photoresponse measurements of $\mathrm{Au} / \mathrm{Cr} / \mathrm{ta}-\mathrm{C} / \mathrm{p}-\mathrm{Si}$ heterojunctions, the original setup of the green laser-line ( $8 \mathrm{~ns}$ pulses at $532 \mathrm{~nm}$ ) with an input impedance of $50 \Omega$ and no additional adjustment circuits was used as described in section 4.4.2, figure 4.12,

The resulting photoresponse characteristic of a typical sample at a reverse-bias of $4 \mathrm{~V}$ and an averaging rate of 512 sweeps is shown in figure 7.4. In order to visualize the 90:10 criteria of rise and decay process, the spectrum was normalized with respect to the maximum photo current. The characteristic exhibits a fast rise time in the range of about $30 \mathrm{~ns}$ while the decay time has a larger value of approximately $100 \mathrm{~ns}$. The exact values of the example at hand are listed inside the corresponding spectrum and are increased by the averaging process due to time-jitter effects compared to a single measurement (marked gray in figure 7.4a). Furthermore, the characteristic exhibits several signal artifacts in the form of ripples which are caused by deviations between the two averaged signals of the actual photoresponse measurement and the dark-current measurement but ultimately result from the subtraction process of those two.

In addition, the photoresponse was investigated as a function of applied reversebias voltage. For comparison reasons, the recorded spectra were normalized with respect to the maximum photoresponse at a reverse-bias voltage of $4 \mathrm{~V}$ and the zero point of time is shifted towards the point of maximum photo current. The resulting characteristics are shown in figure $7.4 \mathrm{~b}$. All of them exhibit fast rise and decay times but increase slightly at lower bias voltages as the effects of time jittering become more significant. As a result, the signal artifacts originating from the induction processes of the laser setup become more distinct at lower bias voltages as the signal to noise ratio decreases. Compared to a reverse-bias voltage of $4 \mathrm{~V}$, primarily dominated by the diode component of the heterojunction according to section 6.3 , the photo-current signal decreases significantly at lower bias voltages and reaches a value of just $5 \%$ of the original signal at zero-bias. Therefore, the photo-current of $\mathrm{Au} / \mathrm{Cr} / \mathrm{ta}-\mathrm{C} / \mathrm{p}-\mathrm{Si}$ heterojunctions can be significantly increased by applying high electric fields at reverse-bias in order to drain the generated charge-carriers. As a result, an absorption of photons primarily in the depletion layer of the MASS heterojunction is a reasonable assumption and in good agreement with theory (see section 3.8). 

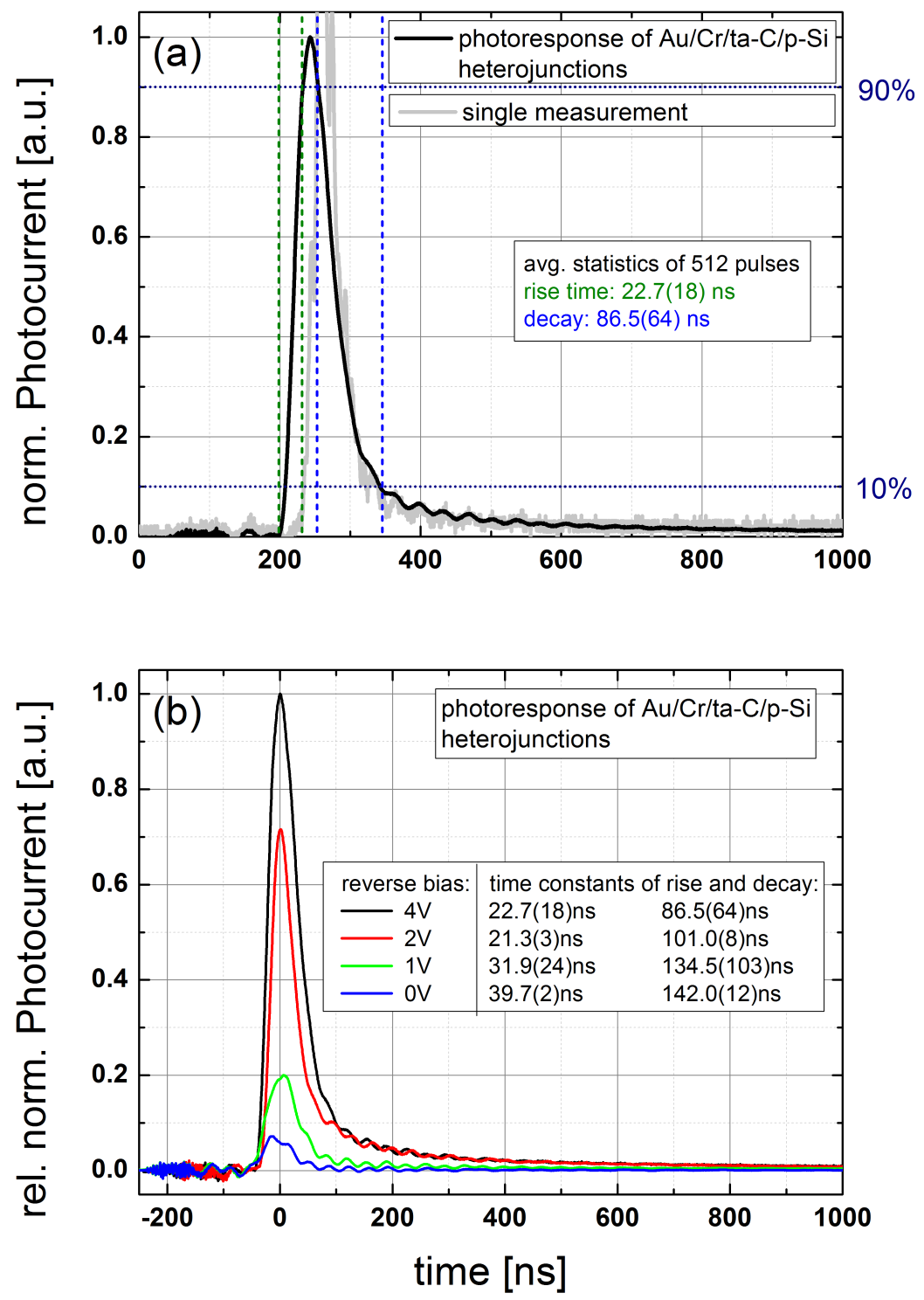

Figure 7.4: Time resolved photoresponse measurements of a typical $\mathrm{Au} / \mathrm{Cr} / \mathrm{ta}-\mathrm{C} / \mathrm{p}-\mathrm{Si}$ heterojunction using the green laser-line ( $8 \mathrm{~ns}$ pulses at $532 \mathrm{~nm}$ ). (a) The normalized photoresponse characteristic exhibits a fast rise time in the range of about $30 \mathrm{~ns}$ while the decay time has a larger value of approximately 100 ns. Both are increased by the averaging process due to time jitter effects compared to a single measurement (gray). (b) Photoresponse characteristics as a function of applied reverse-bias voltage: The maximum photo-current is voltage dependent indicating an absorption inside the depletion layer of the heterojunction located inside the silicon substrate. 


\subsection{2 $\mathrm{Au} / \mathrm{Cr} / \mathrm{t}-\mathrm{BN} / \mathrm{SiC}$ heterostructures}

In a next step, $\mathrm{Au} / \mathrm{Cr} / \mathrm{t}-\mathrm{BN} / \mathrm{SiC}$ heterojunctions were analyzed using the UV-line setup (5 ns pulses at $266 \mathrm{~nm}$, marked gray in figure 4.12) in order to provide enough energy to overcome the band-gap of $\mathrm{SiC}$. In addition, all samples were also exposed to the green laser line for comparison reasons. The corresponding photoresponses of a typical $\mathrm{Au} / \mathrm{Cr} / \mathrm{t}-\mathrm{BN} / \mathrm{SiC}$ heterojunction are shown in figure 7.5. At a reversebias voltage of $5 \mathrm{~V}$, the resulting characteristic exhibits fast rise and decay times of about $12 \mathrm{~ns}$ and $18.5 \mathrm{~ns}$. Again, these values are slightly increased due to time jitter effects and listed in more detail within the plot. Furthermore, the oscillation artifacts resulting from the subtraction of the reference signal are significantly more pronounced leading to a false measurement of the decay times. Therefore, a more accurate value can be estimated using an exponential fit of the decay process. This leads to a more realistic decay constant of $\tau \approx 55(5)$ ns. Furthermore, after exposing the sample to the green laser line $(532 \mathrm{~nm})$ at the same applied voltage, the corresponding characteristic (teal solid line in figure $7.5 \mathrm{a}$ ) does not show any significant sign of a photoresponse (even at an input impedance of $1 \mathrm{M} \Omega$ ) which is in accordance with the predicted transparency of $\mathrm{SiC}$ regarding visible light.

In addition, the photoresponse was also analyzed as a function of applied bias voltage. The results are illustrated in figure 7.5 $\mathrm{b}$. Similar to the ta-C/p-Si based heterojunctions, the photo-current signal is maximal for high electric fields at reverse bias and therefore also primarily dominated by the diode components of the heterojunction (see section 6.4.1). Again, the relative photo-current decreases significantly at lower bias voltages reaching a value of about $12 \%$ of the original signal at zero-bias. Furthermore, the oscillation artifacts become less distinct accompanied by an unexpected decrease of the decay time constants. Both of them might be correlated to each other or be caused by thermal effects at higher bias voltages and corresponding increased photo-currents. One way or another, the precise cause of these effects could not be determined during the scope of this thesis.

Nevertheless, based on the obtained results, the photo-current of $\mathrm{Au} / \mathrm{Cr} / \mathrm{t}-\mathrm{BN} / \mathrm{SiC}$ heterojunctions can also be significantly increased by applying high electric fields at reverse-bias in order to drain the generated charge-carriers and the absorption of photons is primarily located in the depletion layer of the MASS heterojunction. 

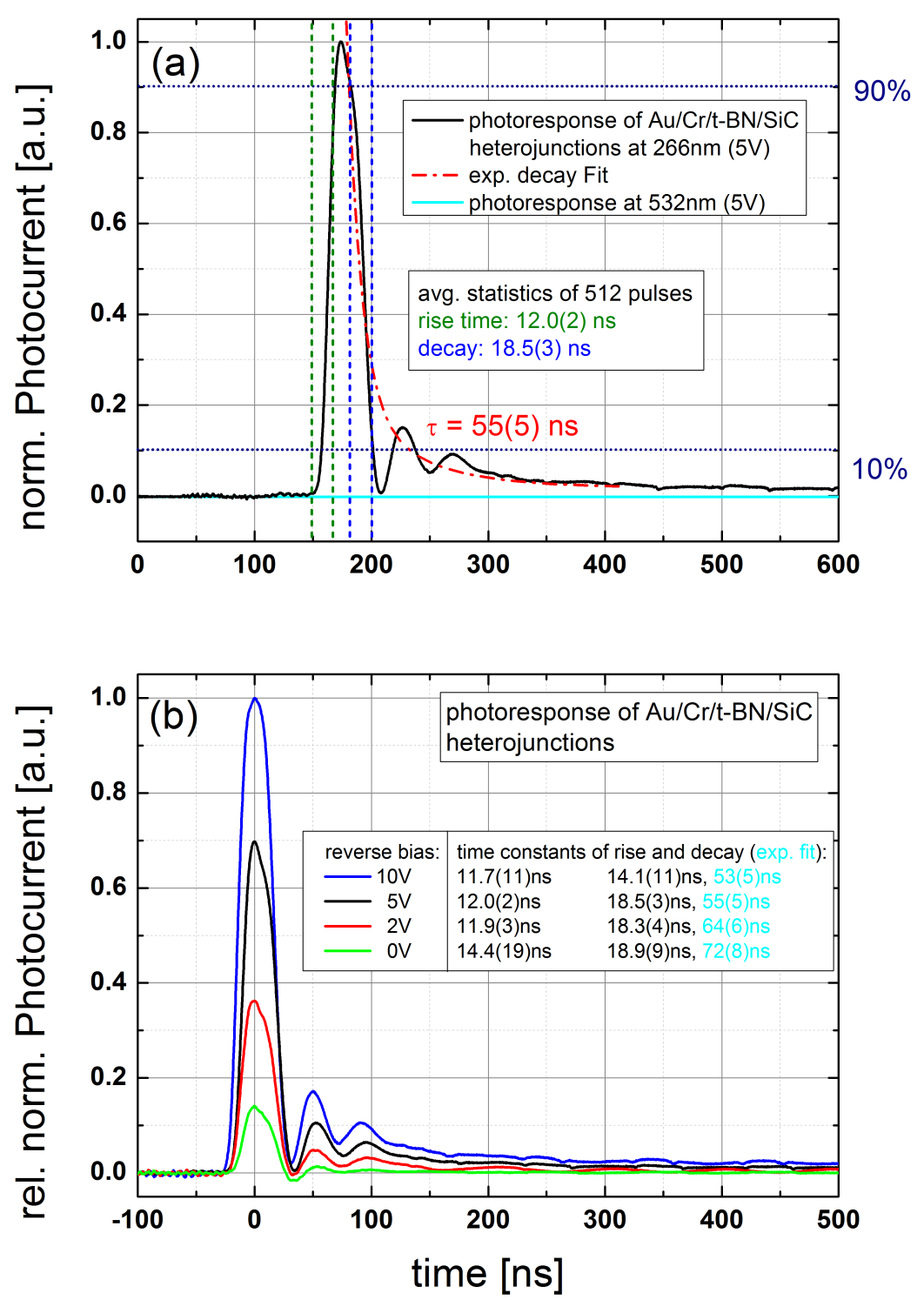

Figure 7.5: Time resolved photoresponse measurements of a typical $\mathrm{Au} / \mathrm{Cr} / \mathrm{t}-\mathrm{BN} / \mathrm{SiC}$ heterojunction using the UV laser-line setup ( $5 \mathrm{~ns}$ pulses at $266 \mathrm{~nm}$ ). (a) The normalized photoresponse characteristic exhibits a fast rise time in the range of about 12 ns while the decay time has a larger value of approximately $55 \mathrm{~ns}$. (b) Photoresponse characteristics as a function of applied reverse-bias voltage: The maximum photo-current is voltage dependent indicating again an absorption inside the depletion layer of the heterojunction located inside the $\mathrm{SiC}$ substrate similar to the ta-C/p-Si MASS diode systems. 


\subsection{3 $\mathrm{Au} / \mathrm{Cr} /$ ta-C/SiC heterostructures}

Furthermore, $\mathrm{Au} / \mathrm{Cr} / \mathrm{ta}-\mathrm{C} / \mathrm{SiC}$ heterojunctions were investigated. The corresponding characteristics of a typical $\mathrm{Au} / \mathrm{Cr} / \mathrm{ta}-\mathrm{C} / \mathrm{SiC}$ heterojunction are illustrated in figure 7.6 and the time-constants are listed within. Compared to the t-BN/SiC system, the normalized photoresponse characteristics are very similar including shape, voltage dependency of maximum photo-currents, rise and decay times as well as the decay constant of $\tau \approx 84(6)$ ns estimated using an exponential fit in order to compensate for the typical signal artifacts which are apparently present in all $\mathrm{SiC}$ based MASS heterojunctions. Therefore, the relative photoresponse does not depend on the composition of the grown thin-film or the interface of the substrate. The absolute photo-currents, on the other hand, differ as the absolute current of each sample is primarily affected by the conductivity of the grown thin-films according to the results of chapter 6 .

However, the consistency of the resulting time constants in both SiC based MASS diode systems indicate almost no interactions between the UV-light of the laser and the grown ta-C as well as t-BN thin-films. Hence, based on the voltage dependency of the photo-current, the absorption of photons takes primarily place in the depletion layer of the heterojunction. 

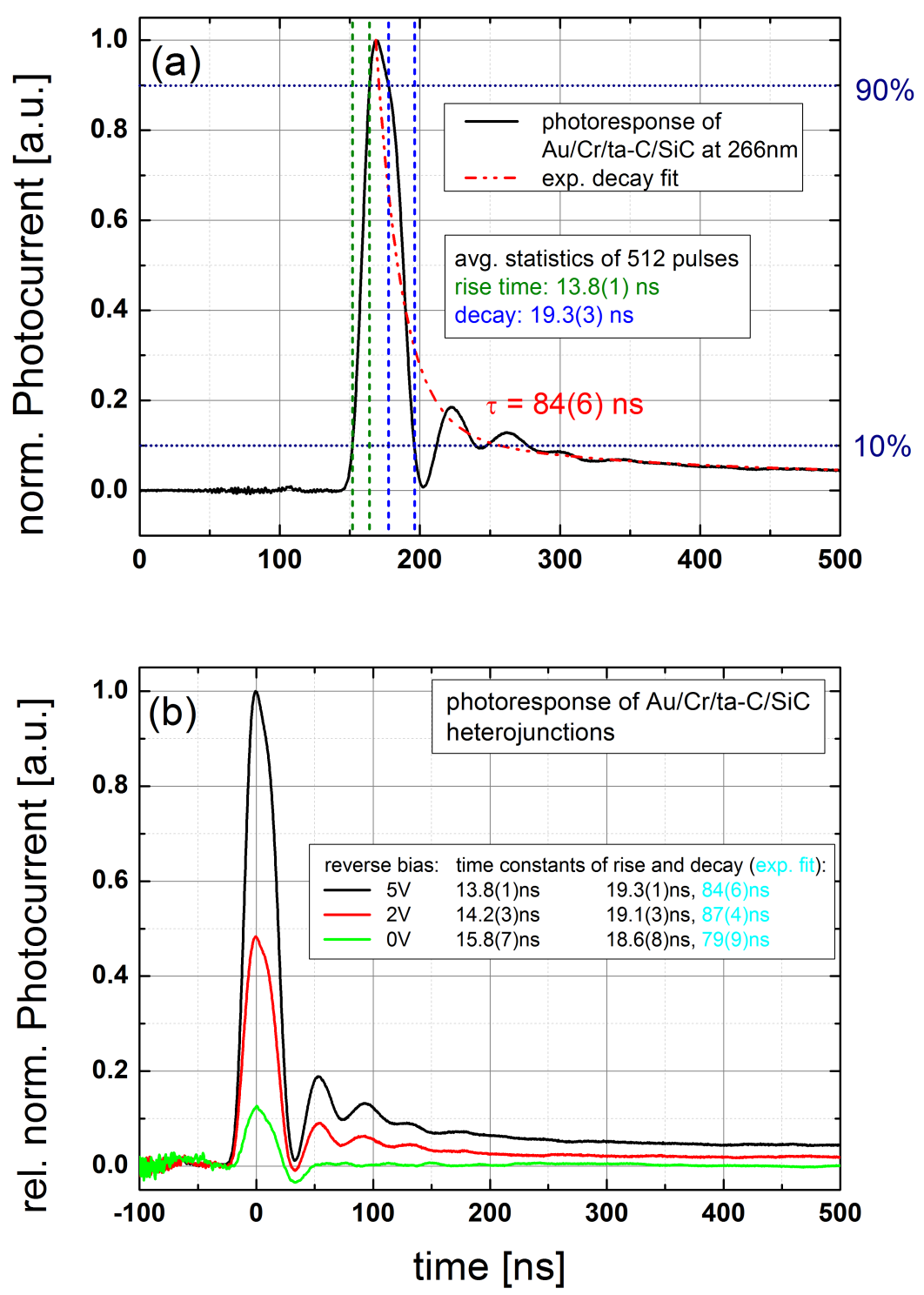

Figure 7.6: Time resolved photoresponse measurements of a typical $\mathrm{Au} / \mathrm{Cr} / \mathrm{ta}-\mathrm{C} / \mathrm{SiC}$ heterojunction using the UV laser-line setup (5 ns pulses at $266 \mathrm{~nm}$ ). (a) The normalized photoresponse characteristic exhibits a fast rise time in the range of about $12 \mathrm{~ns}$ while the decay time has a larger value of approximately $55 \mathrm{~ns}$ very similar to the t-BN/SiC system in figure 7.5 (b) Photoresponse characteristics as a function of applied reverse-bias voltage: The maximum photo-current is voltage dependent indicating again an absorption inside the depletion layer of the heterojunction located inside the $\mathrm{ZnO}$ substrate similar to the other MASS diode systems. 


\subsection{4 $\mathrm{Au} / \mathrm{Cr} / \mathrm{t}-\mathrm{BN} / \mathrm{ZnO}$ heterostructures}

Finally, the photoresponse of $\mathrm{Au} / \mathrm{Cr} / \mathrm{t}-\mathrm{BN} / \mathrm{ZnO}$ heterojunctions was investigated. The corresponding characteristic of a typical sample is shown in figure 7.7. Regarding the normalized photoresponse characteristic at a single reverse-bias voltage of $40 \mathrm{~V}$ (figure $7.7 \mathrm{a}$ ), the corresponding rise time of about $13.5 \mathrm{~ns}$ is quite similar to other MASS diode systems investigated before. The decay time, however, is several orders of magnitude higher and typically exhibits a value in the range of $2-3 \mu \mathrm{s}$.

This slow decay rate can also be observed at lower bias voltages of $30 \mathrm{~V}-10 \mathrm{~V}$ shown in figure 7.7b. Nevertheless, the photo-sensitivity of these heterojunctions decreases towards lower bias voltages and almost vanishes at zero-bias. In comparison to other MASS diode systems, the applied bias voltages are significantly higher due to the low conductivity of the samples (section 6.2). Furthermore, the relative increase in current is significantly larger compared to the other heterojunctions leading to a considerable reduction of oscillation artifacts.

However, the voltage dependence of the particular photo-currents is linked to the voltage drop across the diode components of the heterojunction similar to the other MASS diode systems indicating again an absorption inside the depletion layer of the heterojunction located inside the $\mathrm{ZnO}$ substrate. Therefore, the relatively high decay constants are also likely caused by the $\mathrm{ZnO}$ substrate itself. In this context, the low conductivity of the substrate as well as deep level defects like oxygen vacancies might hamper the relaxation process of charge carriers after exposure resulting in a rather low decay process. 

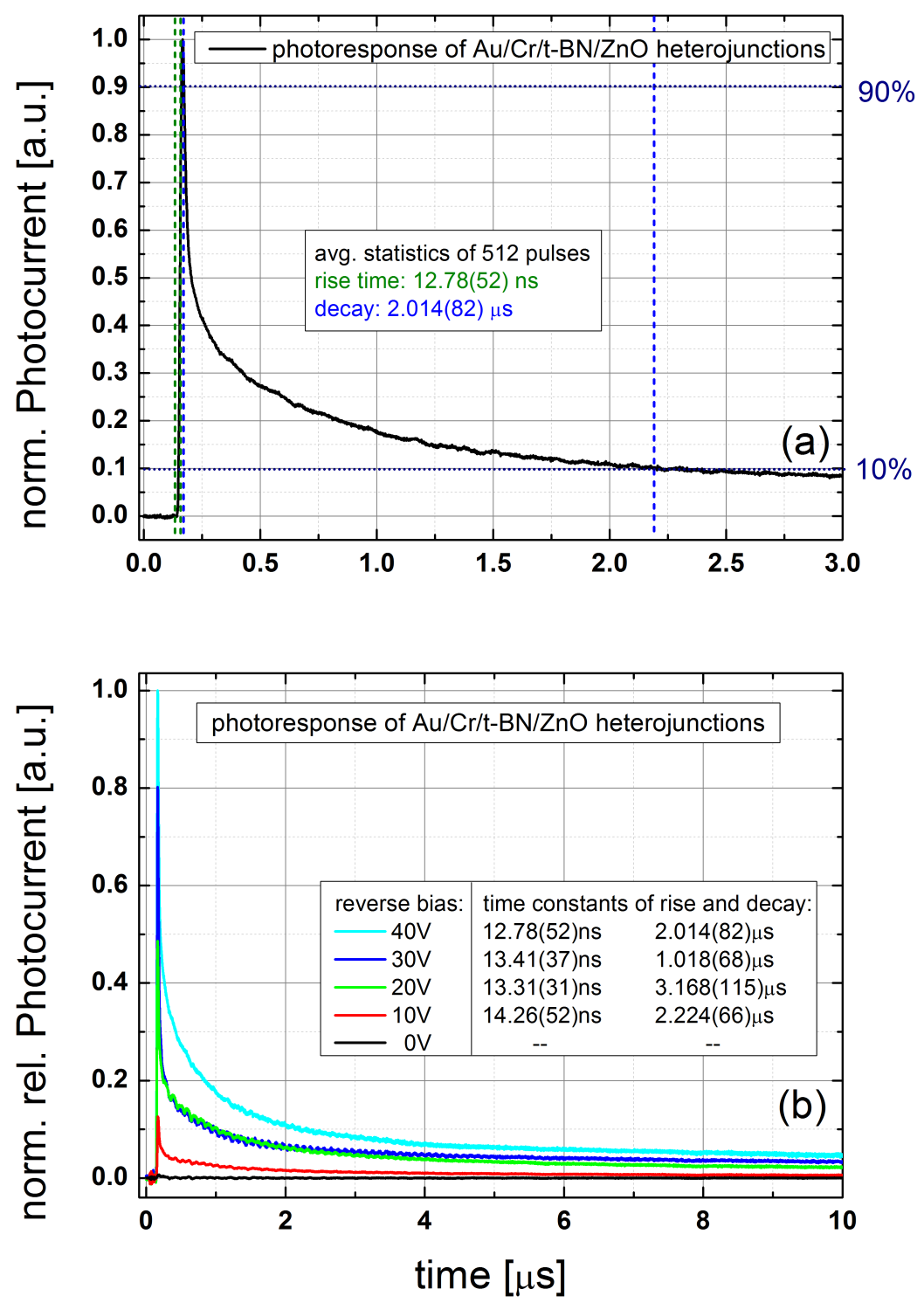

Figure 7.7: Time resolved photoresponse measurements of a typical $\mathrm{Au} / \mathrm{Cr} / \mathrm{t}-\mathrm{BN} / \mathrm{ZnO}$ heterojunction using the UV laser-line setup ( $5 \mathrm{~ns}$ pulses at $266 \mathrm{~nm}$ ). (a) The normalized photoresponse characteristic at a reverse-bias voltage of $40 \mathrm{~V}$ exhibits a rise time of about $13.5 \mathrm{~ns}$. The decay time, however, is several orders of magnitude higher and typically exhibits a value in the range of 2-3 $\mathrm{s}$ s. (b) Photoresponse characteristics as a function of applied reverse-bias voltage: The maximum photo-current is voltage dependent indicating again an absorption inside the depletion layer of the heterojunction located inside the $\mathrm{ZnO}$ substrate similar to the other MASS diode systems. 


\subsection{Summary}

According to case study photoconductivity measurements in section 7.1 using a simple LED setup, all heterojunction systems exhibit a distinct photo-sensitivity when biased in reverse-bias direction while the ta-C and t-BN reference samples do not show any signs of photoresponse. In this context, unusually long decay constants of several hundred seconds obtained for the heterojunction diodes can be linked to thermal excitations of the samples during the measurement and are originally caused by the LED.

Further time-resolved photoresponse measurements on the basis of a pulsed laser assisted setup provide a more standardized analysis technique in terms of illuminated area, light intensity and time-resolution of the recorded photoresponse signals. The signal to noise ratio is very low for photoresponse measurements based on a single

laser pulse. Furthermore, the photoresponse signal is affected by time jitter of the system. Therefore, all recorded datasets are based on the averaged characteristic of 512 measurement sweeps. Despite the effects of time jittering, all MASS heterojunctions exhibit fast rise times in the low two digit nanosecond range indicating an immediate drain of the generated charge carries in each system, especially when reverse-bias voltages are applied. However, the photoresponse decreases significantly at lower voltages reaching a minimum at zero-bias. Based on the results of chapter 6. this effect is accompanied by an increase of fractional bias voltage percentage across the grown thin-films at the expense of the diode components in all investigated MASS heterojunctions, especially around zero-bias. In addition, based on the results of the $\mathrm{SiC}$ based heterojunctions, the photoresponse of the heterojunctions is almost unaffected by the composition of the grown thin-films confirming the results of the case study setup regarding the reference samples grown on low-resistive ntype silicon substrates. Therefore, the distinct voltage dependence of photo-currents in all investigated MASS diode systems indicates an absorption of light inside the depletion-layer region of the particular heterojunctions.

Finally, the obtained decay time constants depend almost entirely on the substrate of the particular heterostructures and do not correspond to the properties of the grown thin-films. In fact, all performed measurements of this thesis do not indicate any interaction of light with the grown thin-films. If any percentage of light is absorbed in the thin-films during illumination, the generated free charge carriers will likely be trapped initially due to the larger number of localized defect states inside ta-C and t-BN.

While $\mathrm{Si}$ and $\mathrm{SiC}$ based MASS diodes exhibit fast decay rates of the excited states in the range of 100-200 ns, the decay rate in $\mathrm{ZnO}$ based samples is several orders of magnitude higher and typically exhibits a value in the range of $2-3 \mu \mathrm{s}$. This fact might be caused by the low conductivity of the substrate as well as deep level defects like oxygen vacancies hampering the relaxation process of charge carriers after exposure. 


\section{Chapter 8}

\section{Impedance spectroscopy}

Based on the results of chapters 5 and 6, the electrical properties (DC) of all fabricated MASS diode heterojunctions of this thesis are substantially affected by the high defect density inside all grown t-BN and ta-C thin films and interface layers inside the heterostructure as well as depleted states inside the space-charge regions of semiconducting substrates. As all the processes are related to trapping or depletion processes of free-charge carriers, the presence of additional capacitive elements inside the heterostructures featuring frequency dependent behavior in an alternate current $(\mathrm{AC})$ setup is a reasonable assumption.

Therefore, the AC-properties of several MASS heterostructures have been investigated by measuring the complex impedance $\underline{Z}(V, \omega)$ as a function of bias voltages as well as of frequency in the range of $20 \mathrm{~Hz}$ to $1 \mathrm{MHz}$ using the setup described in section 4.3. In this context, separate characteristics of the absolute impedance $|Z(V, \omega)|$ and the phase $\theta(V, \omega)$ were recorded as a function of frequency and applied bias voltage in order to identify contributing capacitive components inside the MASS heterostructures in comparison to previous direct current measurements.

\subsection{Fundamentals}

A thorough survey of the theoretical basics regarding $\mathrm{AC}$ conduction in disordered materials like t-BN and ta-C has already been provided in the work of Amani ([135], chapter 2) with basic references to previous works of Jonscher et al. [157-159], Dyre et al. [160] and Scher et al. [161] as well as several other publications [162 164].

\subsubsection{Complex capacitance and constant phase element (CPE)}

In essence, the complex impedance $\underline{Z}$ of a system can be expressed by

$$
Z=R(\omega)+i X(\omega)
$$


where the resistance $\mathrm{R}$ is the real part of the impedance exhibiting no phase-shifting and the reactance $\mathrm{X}$ is the imaginary part of the system. In polar form

$$
Z=R(\omega)+i X(\omega)=|Z| \exp (i \theta(\omega))
$$

the magnitude $|\underline{Z}(\omega)|$ of the impedance as well as the phase difference $\theta(\omega)$ between voltage and current of the investigated system can be extracted. In this context, the corresponding phase shift of an ideal capacitor is $\pi / 2$, while a DC resistor does not exhibit any phase shift due to the infinite impedance of the capacitive element of the system.

In contrast, the dielectric response of amorphous or defect-rich materials exhibiting some kind of hopping or thermally enhanced conduction mechanisms (e.g. FrenkelPoole emission) is a little different as the frequency dependence of such materials is expressed by the "universal power law" $\omega^{\nu-1}$ leading to a so called "universal capacitance" $C_{\nu}$ of the particular system:

$$
C_{\nu}(\omega)=\widetilde{C}(i \omega)^{\nu-1}=\widetilde{C} \omega^{\nu-1}\left[\sin \left(\frac{\nu \pi}{2}\right)-i \cos \left(\frac{\nu \pi}{2}\right)\right]
$$

with a universal power of $0<\nu<1$ and an apparent constant capacitance $\widetilde{C}$ [158, 159]. Moreover, the phase is constant at a value of $-\frac{\nu \pi}{2}$. Therefore, the universal capacitance is also often referred to as constant phase element (CPE).

Furthermore, the reactance of a single capacitor $X_{\mathrm{C}}$ can simply be expressed by the corresponding capacitance and applied frequency $\omega=2 \pi f$

$$
X_{\mathrm{C}}(\omega)=-\frac{1}{\omega C(\omega)} .
$$

If the system is more complex, e.g. due to the presence of an additional small resistor arranged in series $\left(R_{\mathrm{S}}\right)$ as well as a much larger resistor arranged parallel $\left(R_{\mathrm{P}}\right)$ to the capacitor as illustrated in an exemplary test circuit in figure 8.2, the complex capacitance of the system can be written as:

$$
Z(\omega)=Z_{\mathrm{S}}(\omega)+Z_{\mathrm{CP}, \mathrm{P}}(\omega)=R_{\mathrm{S}}+\left(R_{\mathrm{P}}^{-1}-i \omega C_{\mathrm{P}}\right)^{-1}
$$

with

$$
Z_{\mathrm{CP}, \mathrm{P}}(\omega)=\left(R_{\mathrm{P}}^{-1}-i \omega C_{\mathrm{P}}\right)^{-1}
$$

In polar form, the impedance of this parallel arrangement can also be expressed by:

$$
\begin{aligned}
& \left|Z_{\mathrm{P}}(\omega)\right|=\left(R_{\mathrm{P}}^{-2}+\omega^{2} C_{\mathrm{P}}^{2}\right)^{-\frac{1}{2}} \\
& \theta_{\mathrm{P}}(\omega)=\arctan \left(-\omega C_{\mathrm{P}} R_{\mathrm{P}}\right) .
\end{aligned}
$$

The corresponding impedance spectrum of the whole system is illustrated in figure 8.2 based on an ideal capacitor $(\nu=1)$. As long as the resistance of $R_{\mathrm{P}}$ is several

\footnotetext{
${ }^{1}$ The parameter $\nu$ can be derived from the slope of a log-log plot of absolute impedance versus frequency.
} 


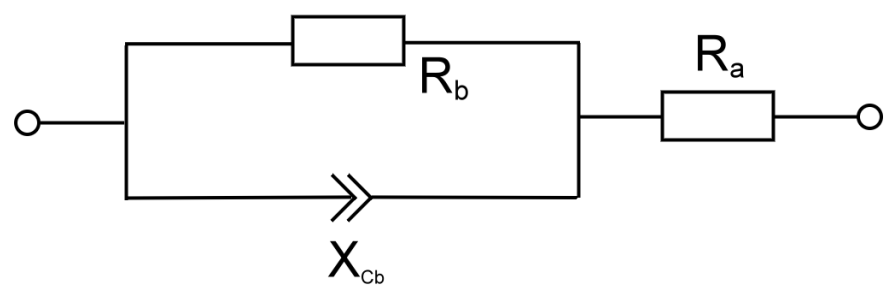

Figure 8.1: Exemplary impedance circuit: the capacitive element of the system $X_{\mathrm{CP}}$ is complemented by a small serial ohmic resistor as well as a much larger parallel ohmic resistor dominating the DC-conduction of the system. The corresponding impedance spectrum of the whole system is illustrated in figure 8.2

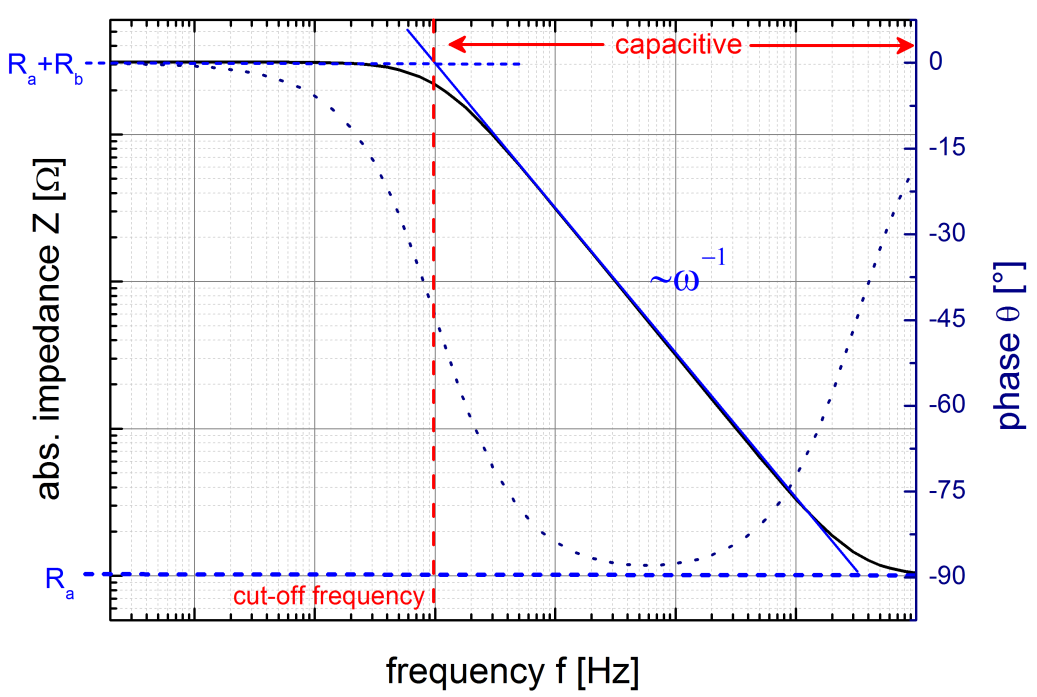

Figure 8.2: Corresponding absolute impedance and phase spectra of the exemplary impedance circuit illustrated in figure 8.2 The characteristics are described in detail in the text below.

orders of magnitude higher than the reactance $X_{\mathrm{CP}}$ of the capacitive element, the absolute impedance as well as the phase of the system are almost constant.

However, as soon as the reactance of the system matches the order of magnitude of $R_{\mathrm{P}}$ at higher frequencies, the absolute impedance of the system starts to decrease considerably. In this context, the related cut-off frequency $\omega_{\text {co }}$ (marked red in figure 8.2) of the system is defined by reaching an impedance of $Z_{\mathrm{CP}, \mathrm{P}}\left(\omega_{\text {co }}\right)=R_{\mathrm{P}} / 2$ accompanied by a decrease in phase down to $-\frac{\pi}{4} 2$ At higher frequencies, the reactance of the system decreases with $\omega^{-1}$ which can easily be obtained from the slope of the absolute impedance characteristic in this frequency range. Therefore, the

\footnotetext{
${ }^{2}$ The cut-off frequency can also be estimated by determining the intercepts of the extrapolated straight lines (blue) related to the capacitive characteristic as well as the resistor $R_{\mathrm{P}}$.
} 
impedance characteristic of the system is "capacitive" in this frequency region and dominated by the capacitive element inside the equivalent circuit. As a result, the corresponding phase decreases down to a value of $-\frac{\pi}{2}$. Furthermore, the decrease in absolute impedance is limited by the serial resistor $R_{\mathrm{S}}$ as soon as the reactance $X_{\mathrm{CP}}$ exhibits the same order of magnitude as $R_{\mathrm{S}}$. As a result, the straight decrease changes towards a constant absolute impedance equal to $R_{\mathrm{S}}$. At the same time, the phase of the system shifts back to a value of zero at much higher frequencies.

\subsubsection{Capacitances of diodes}

In addition, the depletion layer of a diode also forms some kind of capacitive element as opposite charges are accumulated at different sides of the depletion layer and are therefore separated by this high resistive region. As a results, this capacitor is arranged parallel to the particular diode components of a system [165] including leakage resistors.

For an ideal interface, e.g. provided by a MIS-structure (see chapter 1), the frequencydependence would be consistent with a normal capacitor. However, the samples of this work differ considerably from MIS-structures. Based on the intermixing layers or even amorphous interlayer of the heterostructures according to TEM-analyses results in chapter 5 and corresponding increased leakage currents of the diodes according the results of chapter 6, the presence of another constant phase element is a reasonable assumption and was already successfully included into numerical simulations of an FPID-model (see section 6.2, figure 6.10) based on the ta-C/p-Si system in the work of Amani [135] 3

\subsubsection{Parameter determination and numerical simulations}

Therefore, the DC FPID-model was successfully extended with respect to the ACproperties of the grown thin-films as well as the depletion layer of the diode. However, all measurements of that work were only performed at room temperature and on the basis of ta-C/Si based heterojunctions as well as reference samples without performing detailed temperature dependent I-V measurements in DC-mode.

As a result, most of the parameters used in the corresponding numerical simulations of the two systems differ considerably from the results of this work and are, to some extent, contradictory to the consistent DC results of this thesis 4 even though identical samples were used for the ta-C/p-Si system.

In this context, the most striking differences are reliable thickness values of the grown thin-films in this thesis, as demonstrated in chapter 5, as well as the determination of the corresponding average barrier height. Based on the thermal character

\footnotetext{
${ }^{3}$ Furthermore, according to Jonscher et al., frequency dependent characteristics of normal capacitors have only been observed in very few systems [159].

${ }^{4}$ Slight reductions of resistivity compared to the original conduction model components of this work due to bypassing effects of the DC-resistors at higher frequencies were already factored in.
} 
of the related Frenkel-Poole like conduction mechanism the average barrier height can only be determined by temperature dependent measurements as described in section 6.1.1 and an unrestricted variation of the parameter in a numerical simulation eventually leads to an arbitrariness of the resulting fit.

Hence, only the general idea of additionally present constant phase elements inside the corresponding equivalent circuit diagrams were applied to the developed DC-conduction models of this work in order to reevaluate the conformity of these frequency dependent extensions on the basis of a larger number of different heterojunction systems.

In the absence of a temperature dependent impedance measurement setup, the corresponding parameters were solely adapted from the previous DC I-V measurement results of chapter 6 in order to guarantee consistent interpretation results of the recorded characteristics. The parameters related to capacitive elements of the particular conduction models were primarily determined by plotting the absolute impedance as well as the phase as a function of frequency at several constant bias voltages. In this context, the voltages were selected on the basis of the corresponding DC fractional bias voltage characteristics of each heterojunction in chapter 6, The applied simulation software has originally been developed in the work of Amani [135] using the Mathematica environment and was adapted in this thesis in order to match the demands of all the different MASS diode systems and related equivalent circuit diagrams of the following sections, especially the eFPID-model of SiC based samples.

During the actual analysis, the thin-film reference samples were investigated first, similar to the DC measurements, in order to determine basic capacitive parameters of the grown thin-films. 


\subsection{AC properties of thin-film reference samples}

\subsubsection{Metal/ta-C/metallic Si heterostuctures}

Starting with the AC properties of ta-C reference samples on metallic n-type silicon substrates, the corresponding impedance characteristics $|Z(V, \omega)|$ and $\theta(V, \omega)$ (black circles) of a typical ta-C/metallic Si heterostucture are shown in figure 8.3 . Similar to electrical measurements in DC-mode, the characteristics are symmetric at all frequencies and show only slightly increased conductivity at $20 \mathrm{~Hz}$ compared to the DC measurements in chapter 6. At low frequencies, the characteristics are dominated by the DC resistors of the system (see figure 6.3) as the phase is very close to zero for mid-level and high electric fields. However, due to the highly resistive leakage currents of the system around zero-bias, the corresponding complex impedance characteristic exhibits a considerable contribution from a capacitive element in this region even at $20 \mathrm{~Hz}$ leading to the formation of a region with a higher phase shift referred to as "valley" in the following sections.

Furthermore, at higher frequencies exceeding $10 \mathrm{kHz}$ and low electric fields, the phase of the system decreases significantly, accompanied by a frequency dependent increase of conductivity indicating a shift towards a present constant phase element (CPE) of the ta-C thin-film bypassing the DC conductivity of the system due to considerably lower resistivity of the capacitive element.

Based on the low resistivity of the highly doped n-type silicon $(0.005-0.01 \Omega \mathrm{cm})$, the substrate impedance is negligible similar to DC conduction. In addition, capacitive elements originating from the substrate are also highly unlikely in the absence of any Schottky barriers.

Therefore, the recorded characteristics can primarily be linked to the AC properties of the ta-C thin-film, and the DC Frenkel-Poole model of this work (see figure 6.3) can be extended by an additional constant phase element as shown in figure 8.4. Moreover, the FP-model has also been extended by two additional ohmic resistors $R_{\mathrm{S}}$ and $R_{\mathrm{C}}$. The serial resistor $R_{\mathrm{S}}$ was added in order to cover possible contact resistances interfering with the complex impedance characteristics of the thin-films at high frequencies. However, it is negligible regarding the corresponding resistances of the reference samples in this thesis. The ohmic resistor $R_{\mathrm{C}}$, on the other hand, covers the theoretical finite saturation resistance of the grown thin-films at very high electric fields.

The resulting numerically simulations (red circles) are also illustrated in figure 8.3 and are basically in good agreement with measured datasets (black circles). In this context, the simulation is in almost perfect agreement with the recorded datasets at high electric fields indicating a successful implementation of the $\mathrm{AC}$ properties related to the Frenkel-Poole like conduction of the grown ta-C thin-film dominating at higher bias voltages according to chapter 6. Therefore, the basic parameters of the constant phase element can be extracted leading to estimates of universal power $\nu \approx 0.84$ and capacitance of the amorphous layer $\widetilde{C} \approx 16.5 \mathrm{nF}$.

However, the simulated phase differs significantly from the recorded data around 

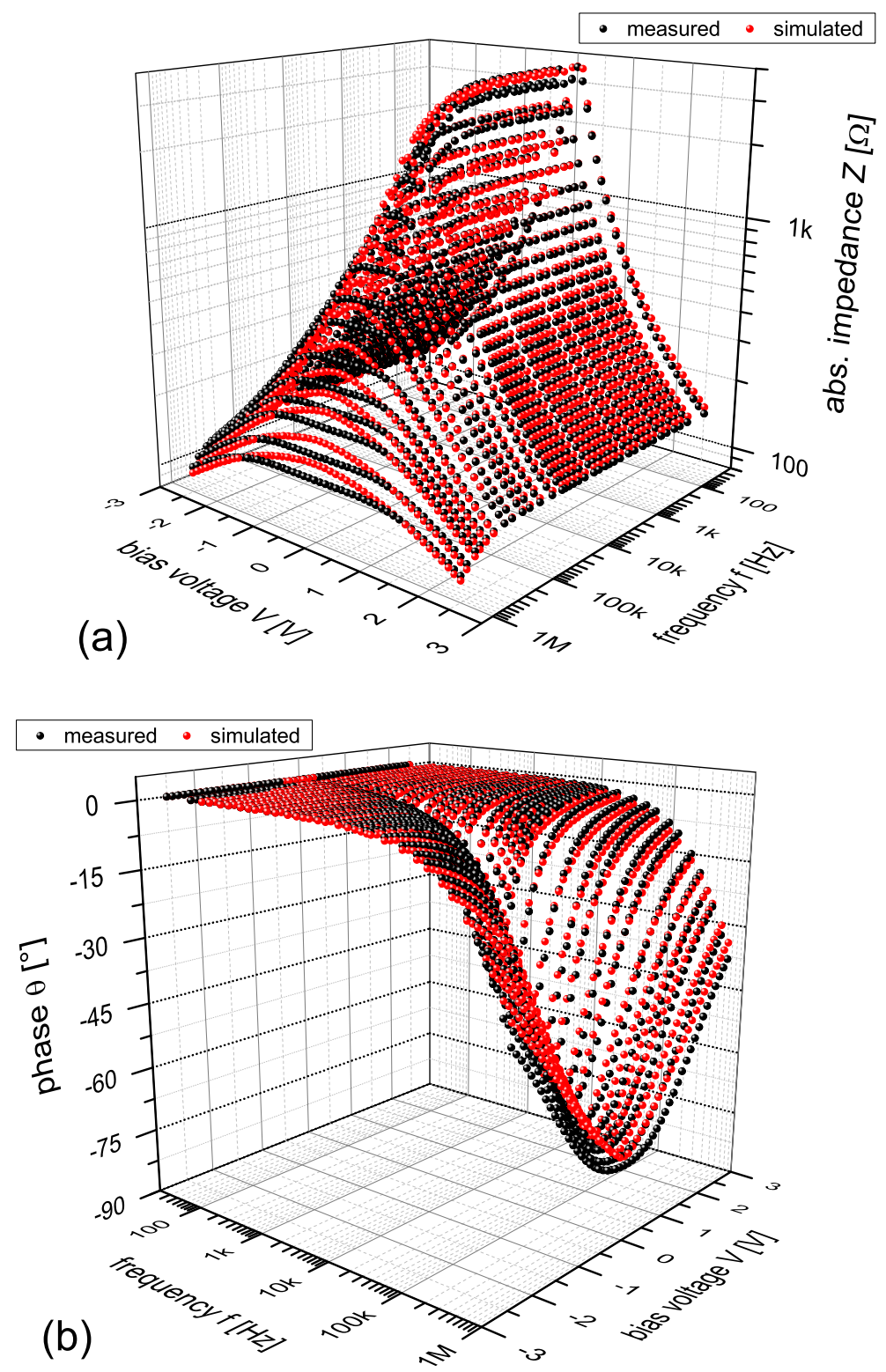

Figure 8.3: Typical complex impedance measurement of a ta-C reference sample (black circles) including separate characteristics of the absolute impedance $|Z(V, \omega)|$ (a) and the corresponding phase $\theta(V, \omega)(\mathrm{b})$. The simulated datasets of the AC FP-model (red circles) according to figure 8.4 are basically in good agreement with the recorded characteristics at high voltages but differ around zero-bias at higher frequencies.

zero-bias indicating the presence of another capacitive element related to the leakage current of the thin-film.

This is also illustrated using cross-sections of the complex impedance characteristics at zero-bias as well as at maximum applied bias voltage. The corresponding characteristics of absolute impedance $|Z(\omega)|$ (black) and corresponding phase $\theta(\omega)$ (blue) 


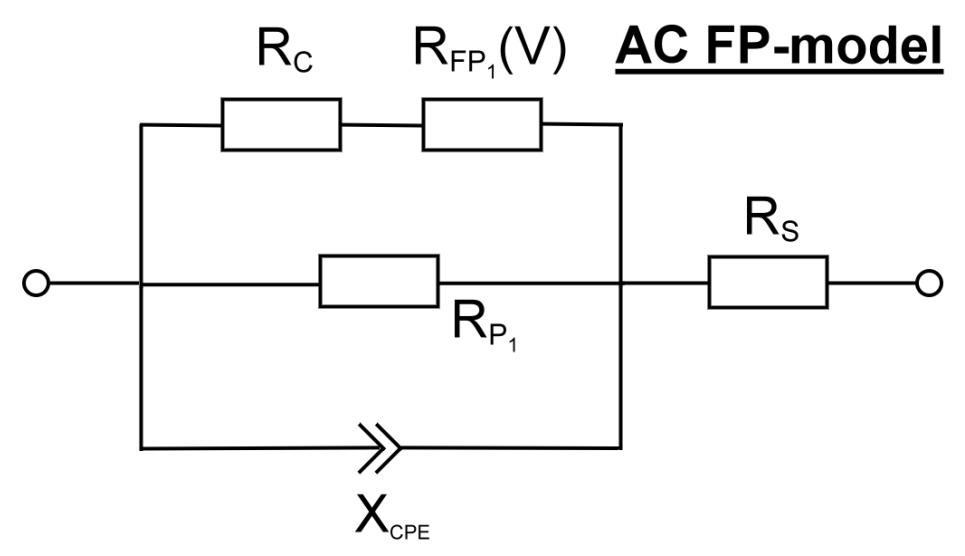

Figure 8.4: Equivalent circuit diagram for AC impedance characteristics of amorphous thin-film reference samples. Compared to the DC model in figure 6.3 an additional constant phase element (CPE) has been added parallel to the FP-resistor representing the capacitance of the amorphous thin-film.

as a function of frequency at these constant voltages are shown in figure 8.5. As the leakage resistor of the grown-films is likely based on hopping and/or tunneling conduction mechanisms, the corresponding capacitor might also differ significantly from the original properties of a voltage-independent CPE.

In this context, the absolute impedance characteristics in figure 8.3 additionally exhibit a progressively widening saturation region around zero-bias at higher frequencies exceeding $10 \mathrm{kHz}$ and correspond to the shift towards the CPE of the thin-film. Therefore, this phenomenon can be directly linked to the capacitive components of the system. As a result, the model might be improved due to implementation of an additional leakage capacitance or a voltage dependent constant phase element of the grown thin-films related to the low-field leakage currents.

However, despite the absence of a temperature dependent AC-setup as well as detailed information about the origin of the DC leakage resistor, the AC FP-model in figure 8.4 is a good approximation regarding the $\mathrm{AC}$ properties of the grown ta-C thin-films. 


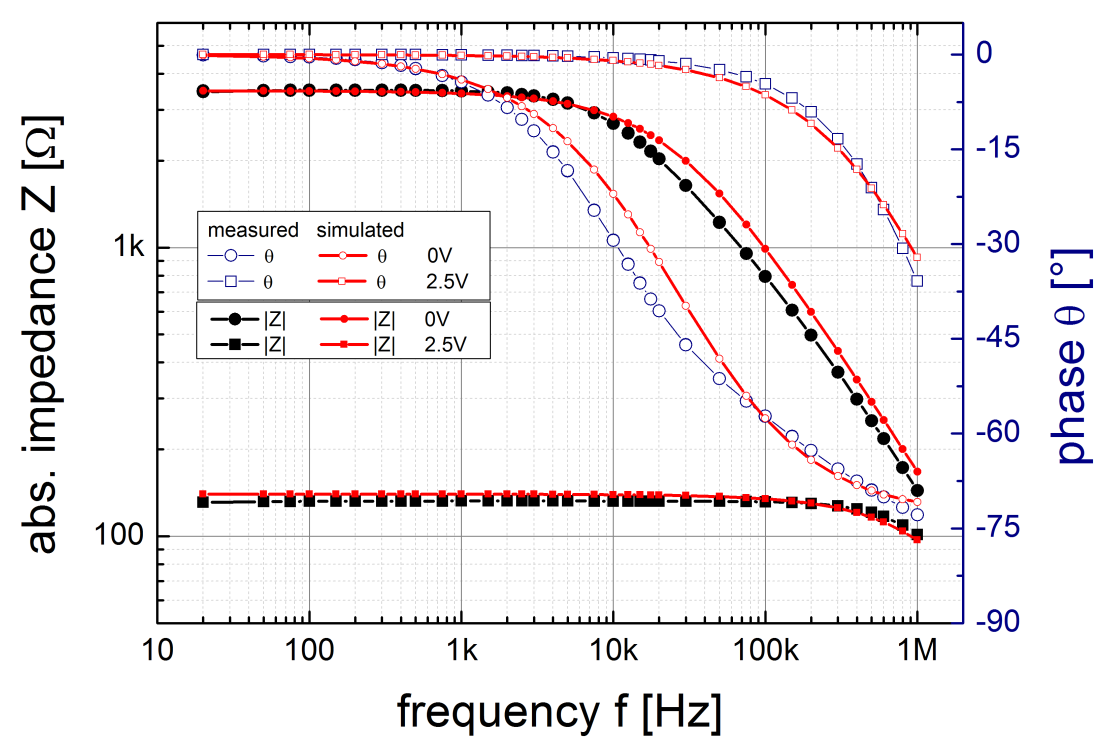

Figure 8.5: Absolute impedance and corresponding phase of a typical ta-C reference sample at constant voltages of zero-bias (circles) as well as of maximum applied bias voltage of $2.5 \mathrm{~V}$ (squares) extracted from figures $8.3 \mathrm{a}$ and $\mathrm{b}$.

\subsubsection{Metal/t-BN/metallic Si heterostuctures}

In a next step, the t-BN reference samples on metallic n-type silicon substrates were investigated with respect to their AC properties. The corresponding impedance characteristics $|Z(V, \omega)|$ and $\theta(V, \omega)$ (black circles) of a typical t-BN/metallic $\mathrm{Si}$ heterostucture are shown in figure 8.6 including corresponding numerical simulations (red circles) of the AC Frenkel-Poole model according to figure 8.4.

Again, the symmetric characteristics exhibit only slightly increased conductivity at $20 \mathrm{~Hz}$ compared to DC measurements in section 6.1.1. In comparison to ta-C reference samples, the cut-off frequency at high electric fields of about $1 \mathrm{kHz}$ is significantly lower. This is primarily caused by the much higher resistivity of the t-BN thin-films compared to the corresponding capacitive component of ta-C reference samples according to the characteristics shown in figure 8.3. At zero-bias, this effect is even more pronounced as the phase is already shifted away from a pure DC related behavior even at $20 \mathrm{~Hz}$. In addition, the voltage dependency of the characteristics vanishes around $2-3 \mathrm{kHz}$ indicating a complete bypass of the $\mathrm{DC}$ resistors of the system.

Nevertheless, at high electric fields, the numerical simulations of the absolute impedance as well as the corresponding phase characteristics are in very good agreement with the recorded data. Therefore, the basic parameters of the constant phase element can be extracted leading to estimates of universal power $\nu \approx 0.94$ and capacitance of the thin-film layer $\widetilde{C} \approx 1.8 \mathrm{nF}$. Compared to ta-C thin-films the 

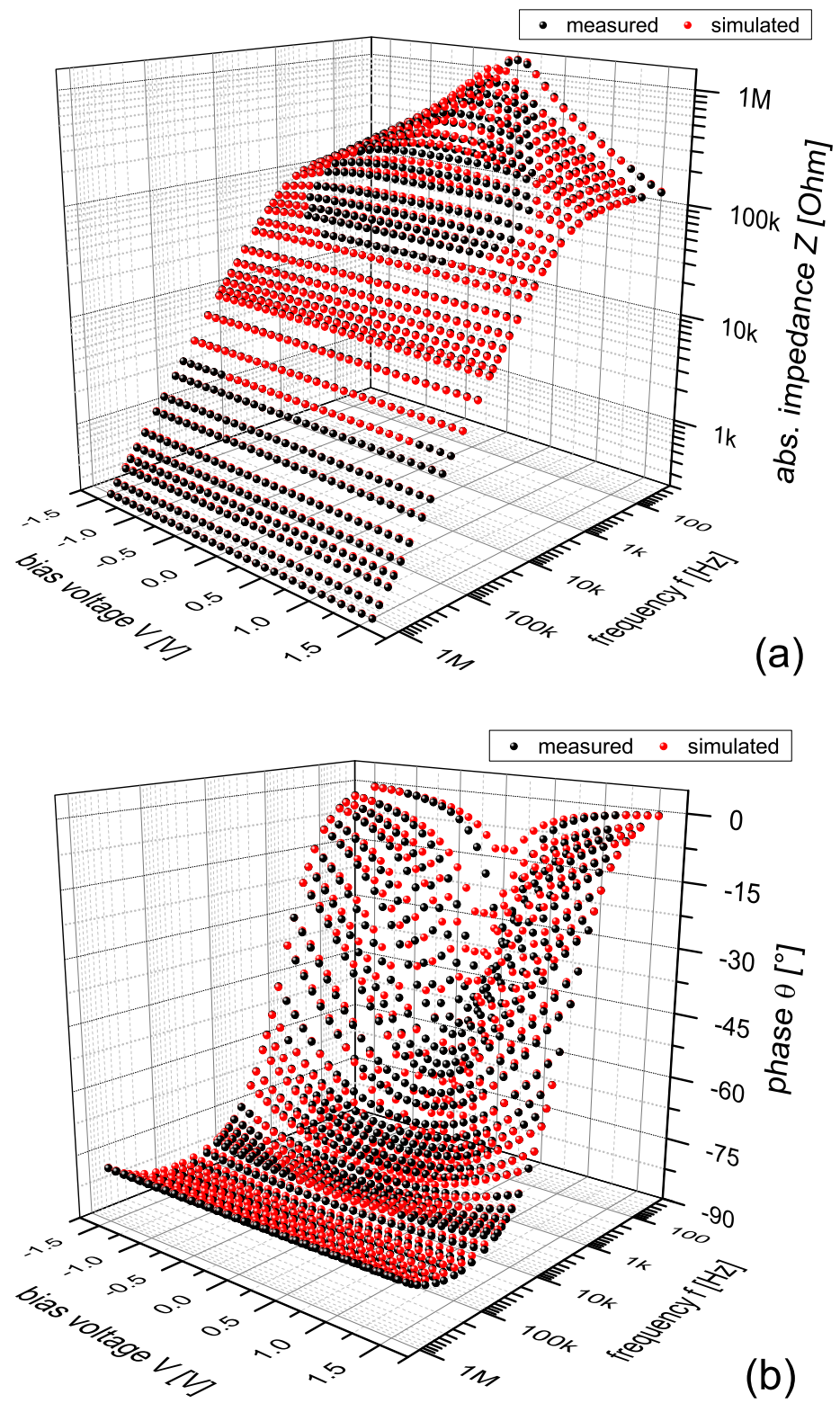

Figure 8.6: Typical complex impedance measurement of a t-BN reference sample (black circles) including separate characteristics of the absolute impedance $|Z(V, \omega)|$ (a) and the corresponding phase $\theta(V, \omega)(\mathrm{b})$. The simulated datasets of the AC FP-model (red circles) according to figure 8.4 are basically in good agreement with the recorded characteristics at high voltages.

capacitance is about one order of magnitude lower while the value of the universal power in t-BN indicates the presence of a more "ideal" capacitor likely caused by the high resistivity of the dielectric material.

Despite the successful application of the AC FP-model at high electric-fields, the simulated phase differs significantly from the actual values below $10 \mathrm{kHz}$ around 
zero-bias accompanied by an overestimation of absolute impedance below $1 \mathrm{kHz}$ similar to the effects observed for ta-C reference samples. Therefore, the presence of an additional capacitive component dominating around zero-bias is a reasonable assumption for t-BN reference samples as well.

These results are again emphasized by cross-sections of the characteristics in figure 8.6 and illustrated in figure 8.7 based on absolute impedance (black) and corresponding phase characteristics (blue) as a function of frequency at constant voltages of zero-bias (circles) as well as of maximum applied bias voltage of $1.5 \mathrm{~V}$ (squares). Nevertheless, the agreement of simulated and recorded data at higher frequencies around zero-bias implies that the observed discrepancies are merely restricted to the transition region between the dominating $\mathrm{DC}$ and $\mathrm{AC}$ conduction properties of the grown thin-films, at least for highly resistive thin-film materials such as t-BN.

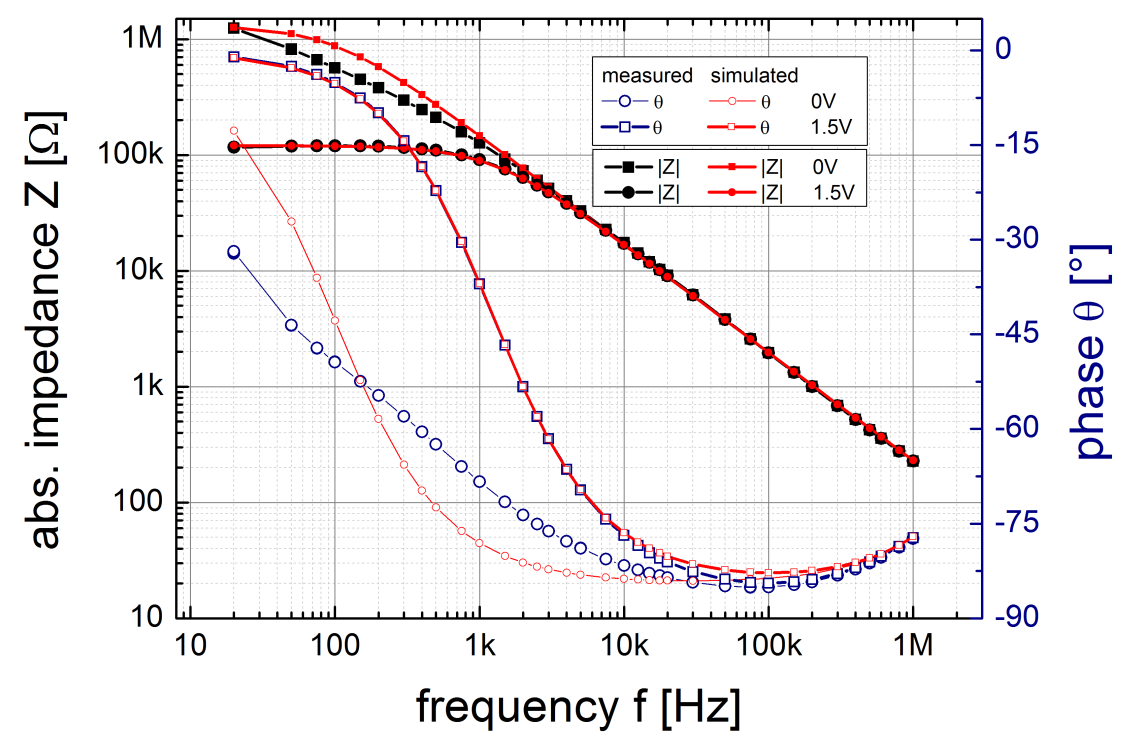

Figure 8.7: Absolute impedance (black) and corresponding phase (blue) of a typical t-BN reference sample as a function of frequency at constant voltages of zero-bias (circles) as well as of maximum applied bias voltage of $1.5 \mathrm{~V}$ (squares) extracted from figures $8.6 \mathrm{a}$ and $\mathrm{b}$. The corresponding numerical simulations are in almost perfect agreement at maximum applied bias voltage but differ considerably at zero-bias below frequencies of $10 \mathrm{kHz}$. 


\subsection{AC properties of MASS heterojunctions}

Based on the results of the thin-film reference samples, the constant phase element $X_{\mathrm{CPE}}$ related to the ta-C or t-BN layer has been included as an extension into the DC Frenkel-Poole and ideal diode models (FPID-models, shown in figures 6.10 and 6.27) including the slight adjustment of ohmic serial resistors of the system. Furthermore, an additional constant phase element $X_{\mathrm{CPE}, \mathrm{D}}$ related to the depletion
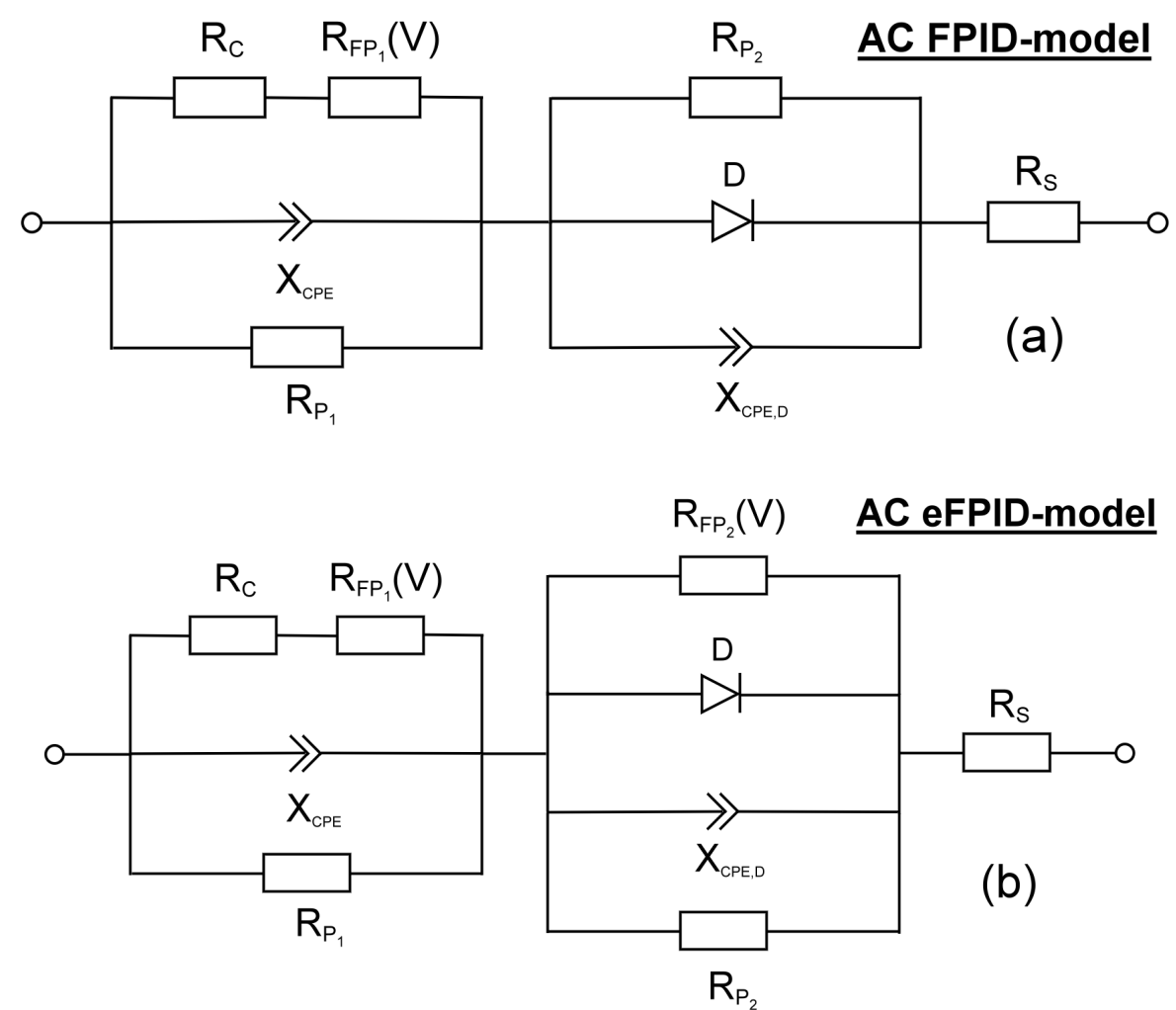

Figure 8.8: Equivalent circuit diagrams of the DC Frenkel-Poole and ideal diode models (FPIDmodel (a), eFPID-model (b)) extended with respect to the AC properties of the individual components. A constant phase element $X_{\mathrm{CPE}}$ related to the ta-C or t-BN layer has been included into both Frenkel-Poole and ideal diode models including the slight adjustment of ohmic serial resistors of the system. Furthermore, an additional constant phase element $X_{\mathrm{CPE}, \mathrm{D}}$ related to the depletion layer of the MASS diodes according to section 8.1 was also added to the equivalent circuits of the conduction models. The remaining components are identical to the original DC conduction models in figures 6.10 and 6.27 .

layer of the MASS diodes according to section 8.1 was also added to the equivalent circuits of the conduction models. The resulting equivalent circuits diagrams are shown in figures $8.8 \mathrm{a}$ (AC FPID-model) and 8.8b (AC eFPID-model).

Again, due to the lack of temperature dependent measurements, the characteristic parameters of each MASS diode system such as curvature, dielectric constants, barrier heights and parasitic resistances have been adapted from the different values obtained from numerical simulations of the DC conduction models. 


\subsection{1 ta-C/p-Si heterostructures}

Starting with the AC properties of $\mathrm{Au} / \mathrm{Cr} / \mathrm{ta}-\mathrm{C} / \mathrm{p}-\mathrm{Si}$ heterojunctions, the corresponding impedance characteristics $|Z(V, \omega)|$ and $\theta(V, \omega)$ (black circles) are illustrated in figure 8.9 including numerical simulations (red circles) of the AC FPIDmodel according to figure 8.8 a . All absolute impedance characteristics show a pronounced rectifying behavior in the whole frequency spectrum accompanied by distinct differences regarding cut-off frequencies of forward- $(1 \mathrm{MHz})$ and reverse-bias direction $(1 \mathrm{kHz})$ of the MASS diode due to different character of the corresponding regions inside the heterostructure regarding the structural properties as well as related resistivity and capacitance.

Focusing on the numerical simulations of the AC FPID-model, they are basically in good agreement with the recorded datasets as long as the characteristics are dominated by the DC resistors of the system, more precisely at low frequencies of $f \leq 20 \mathrm{kHz}$ at forward-bias and $f \leq 2 \mathrm{kHz}$ at reverse-bias.

At higher frequencies, on the other hand, the simulated datasets deviate progressively from the recorded datasets around zero-bias as well as at low- and mid-level electric fields in the rectifying region of the characteristics. With respect to the arrangement of components in terms of the equivalent circuit, these discrepancies are mainly caused by an insufficient simulation regarding the voltage dependencies of the capacitive elements besides the consistent DC resistor arrangement.

However, at high electric fields, the numerical simulations are again in accordance with the recorded measurements which can be emphasized by additional crosssections of the characteristics at maximum applied bias voltages in forward- and reverse-bias direction. The corresponding characteristics of absolute impedance $|Z(\omega)|$ (black) and corresponding phase $\theta(\omega)$ (blue) as a function of frequency at these constant voltages are illustrated in figure 8.10 .

The slight discrepancy at maximum reverse-bias exceeding a frequency of $50 \mathrm{kHz}$ is mainly caused by interference with the constant phase element of the ta-C thin-film. In addition, roughly estimated values of basic parameters related to the constant phase elements can be extracted from these numerical simulations leading to values of 0.86 (ta-C) and 0.92 (diode) for the universal power $\nu$ and capacitances of $\widetilde{C}_{\mathrm{FP}} \approx 3.5 \mathrm{nF}$ (ta-C) and $\widetilde{C}_{\mathrm{D}} \approx 90 \mathrm{pF}$ (diode). Compared to ta-C reference samples in section 8.2.1, the universal power is almost identical while the capacitance of the ta-C thin-film is considerably lowered by a factor of about 5 .

Finally, the absolute impedance characteristics exhibit some kind of abrupt changes in frequency dependence at low electric fields in forward-bias in form of a small step around a frequency of $100 \mathrm{kHz}$ (blue arrows in figure 8.3). The small step in absolute impedance is accompanied by a pronounced valley in phase characteristics at low electric fields similar to the one observed in ta-C reference samples. However, this minimum progressively shifts from about $-0.5 \mathrm{~V}$ at $1 \mathrm{kHz}$ to around $-2 \mathrm{~V}$ at $1 \mathrm{MHz}$ while the valleys of the numerical simulated datasets of the AC FPID-model are fixed at a constant value of $-0.5 \mathrm{~V}$ and exhibit considerably larger phase shifts. In addition, the observed voltage dependency is very similar to the widening process of 

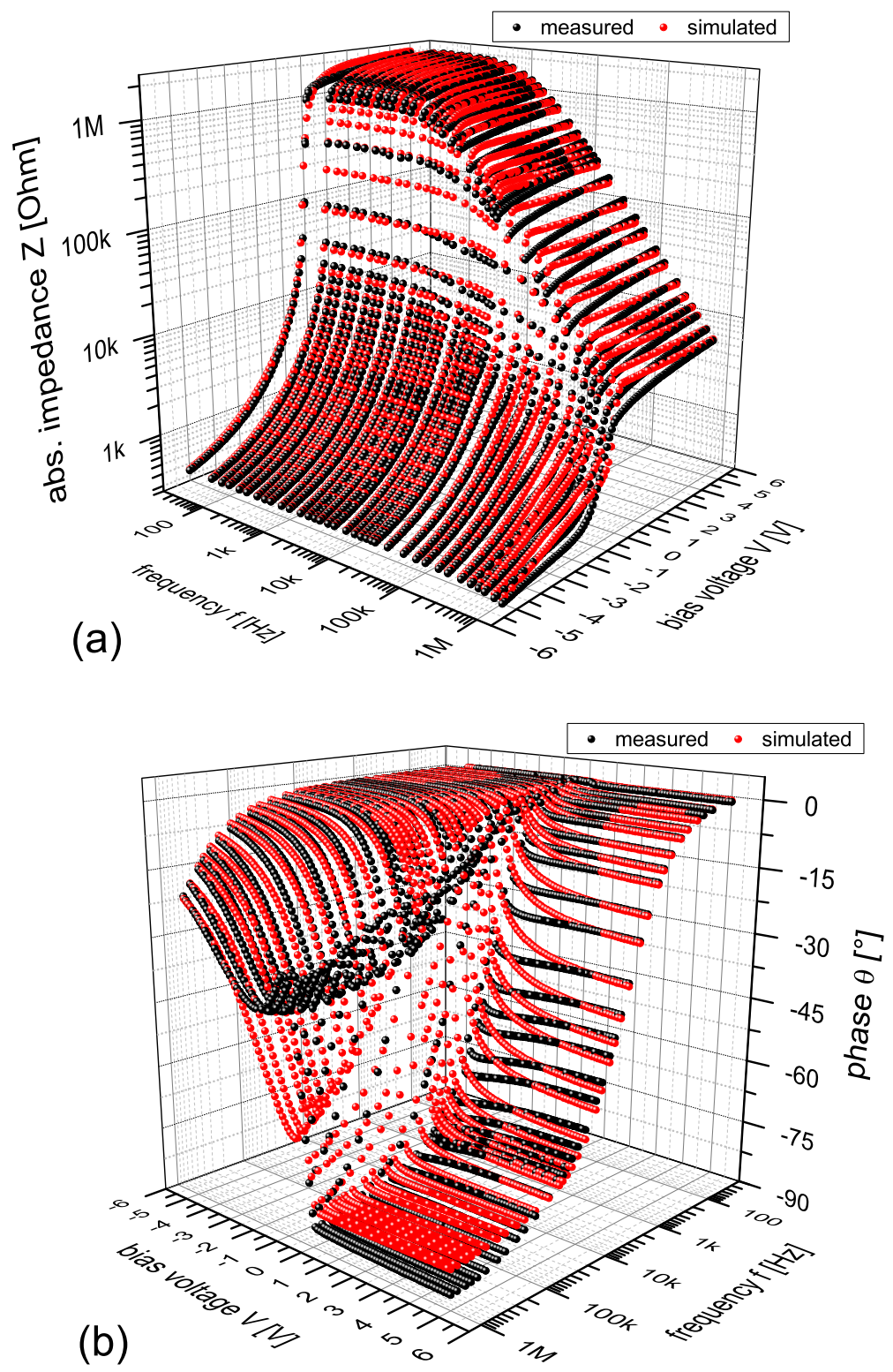

Figure 8.9: Typical complex impedance characteristic of a $\mathrm{Au} / \mathrm{Cr} / \mathrm{ta}-\mathrm{C} / \mathrm{p}-\mathrm{Si}$ heterojunction (black circles) including separate characteristics of the absolute impedance $|Z(V, \omega)|$ (a) and the corresponding phase $\theta(V, \omega)(\mathrm{b})$. The simulated datasets of the AC FP-model (red circles) according to figure $8.8 \mathrm{a}$ are basically in good agreement with the recorded characteristics at high voltages.

the saturation limit of ta-C reference samples with increasing frequency in figure 8.3 of section 8.2.1. Although this context might be just coincidental, the shift of the phase minimum accompanied by a deviation of the applied conduction model still indicates some kind of voltage dependence regarding the corresponding capacitive element dominating this frequency and bias voltage domains. 


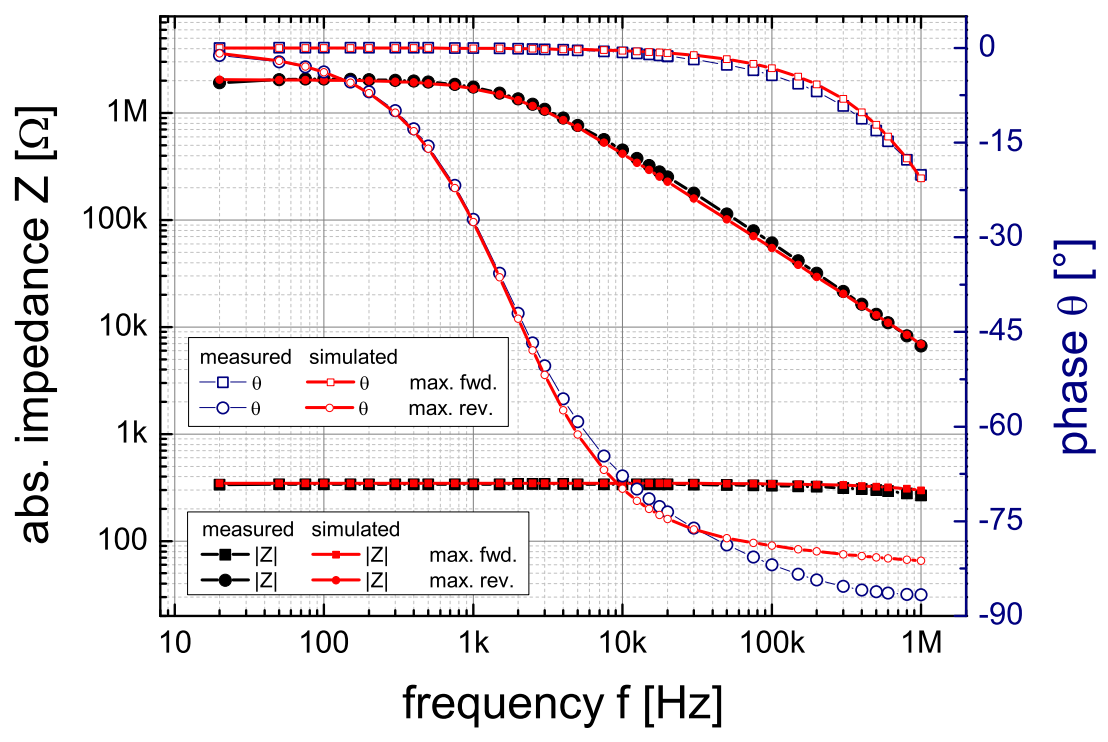

Figure 8.10: Absolute impedance $|Z(\omega)|$ (black) and corresponding phase $\theta(\omega)$ (blue) of a $\mathrm{Au} / \mathrm{Cr} / \mathrm{ta}-\mathrm{C} / \mathrm{p}-\mathrm{Si}$ heterojunction as a function of frequency at maximum applied bias voltages of $\pm 5 \mathrm{~V}$ in forward- (squares) and reverse-bias direction (circles) extracted from figures $8.9 \mathrm{a}$ and b. 


\subsection{2 ta-C/SiC heterostructures}

In a next step, the $\mathrm{AC}$ properties of $6 \mathrm{H}-\mathrm{SiC}$ based heterojunctions were investigated. As illustrated in figure $8.8 \mathrm{~b}$, the predicted AC eFPID-model is rather complex leading to an interference of seven different resistors and capacitive elements around zero-bias, at least at low frequencies. Therefore, the system of equations is mas-
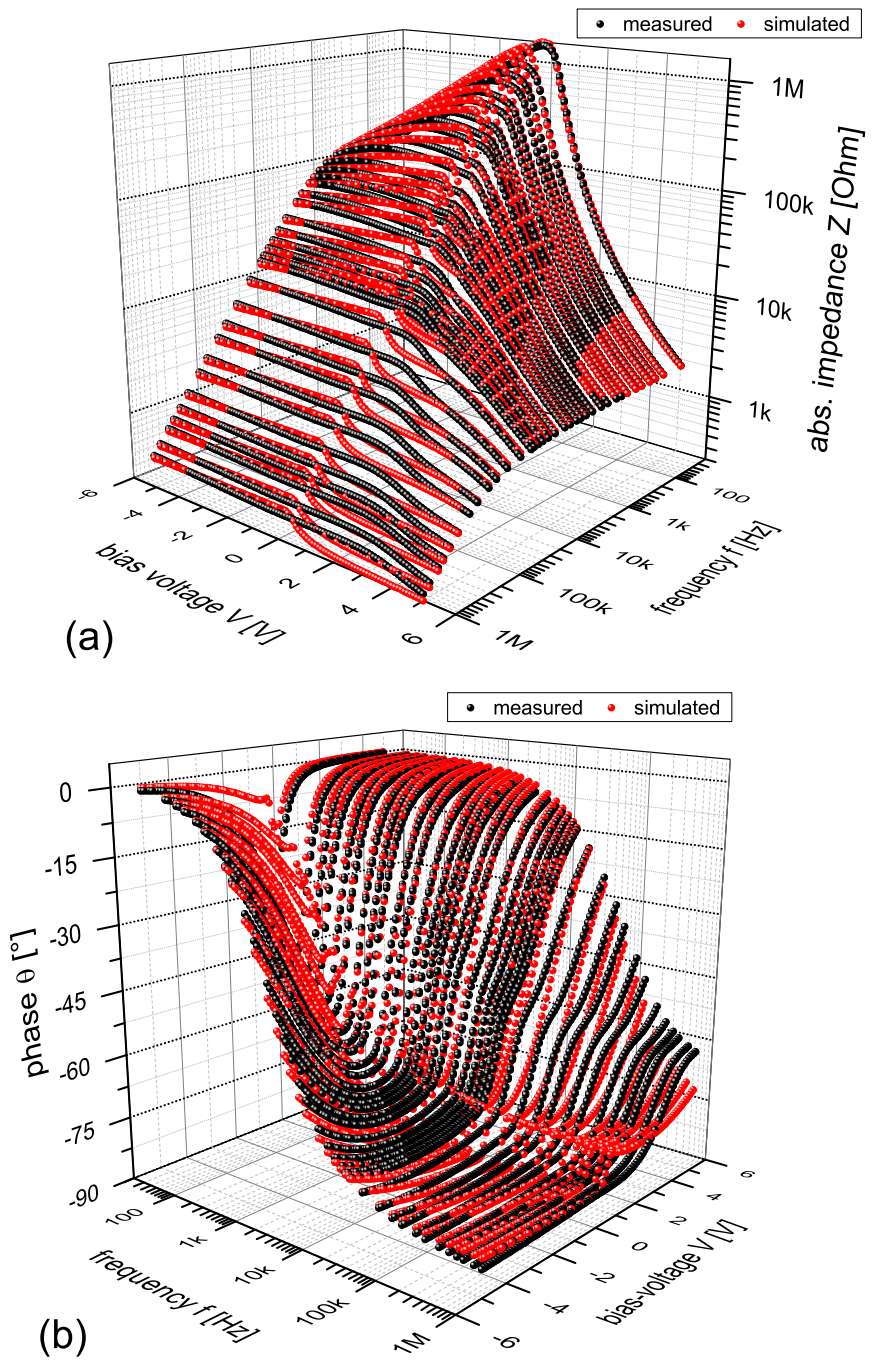

Figure 8.11: Typical complex impedance characteristic of a $\mathrm{Au} / \mathrm{Cr} / \mathrm{ta}-\mathrm{C} / \mathrm{SiC}$ heterojunction (black circles) including separate characteristics of the absolute impedance $|Z(V, \omega)|$ (a) and the corresponding phase $\theta(V, \omega)(\mathrm{b})$. The simulated datasets of the AC FP-model (red circles) according to figure $8.8 \mathrm{~b}$ are basically in accordance with the recorded characteristics at high voltages.

sively over-determined in terms of free-parameters and reasonable results of the numerical calculations depend almost entirely on the previous DC-measurements in section 6.4 as well as on suitable initial values of universal power and capacitances. Nevertheless, starting with $\mathrm{Au} / \mathrm{Cr} / \mathrm{ta}-\mathrm{C} / \mathrm{SiC}$ heterojunctions, the corresponding 
impedance characteristics $|Z(V, \omega)|$ and $\theta(V, \omega)$ (black circles), illustrated in figure 8.11, can basically be matched by the numerical simulations (red circles) of the $\mathrm{AC}$ eFPID-model according to figure 8.8 $\mathrm{b}$, especially again at high electric fields in forward- as well as reverse-bias direction of the diode.

On the other hand, there are also considerable deviations between the numerical simulations and the recorded data at low electric fields all over the investigated frequency spectrum especially around zero-bias. The discrepancies at forward-bias direction are primarily based on an progressively arising saturation effect of the absolute impedance around zero-bias and frequencies above approximately $5-10 \mathrm{kHz}$. In this context, the shape of the absolute impedance characteristics as well as the frequency range are almost identical compared to the effects observed for ta-C reference samples in section 8.2.1 and also slightly for t-BN reference samples in section 8.2.2 indicating a point of origin inside the grown thin-films or at their interfaces. Furthermore, the effect is also accompanied by the formation of "plateau"-region inside the phase characteristics which cannot be successfully fitted by the existing conduction model.

In addition, despite a suitable modulation of the corresponding phase at reverse-bias matching the recorded datasets with a low margin of error (figure $8.11 \mathrm{~b}$ ), the discrepancies between the numerical simulation data and the actual absolute impedance characteristics at reverse-bias (figure 8.11a) are likely caused by a failure of the AC eFPID-model in terms of combined frequency and voltage dependencies regarding the capacitive diode components.

Eventually, the capacitive properties of the depletion layer inside these heterojunctions will essentially be affected by the voltage dependent diode leakage resistor exhibiting Frenkel-Poole like conduction in corresponding DC-measurements (see section 6.4.2). Therefore, the electrical properties of the amorphous interlayer in SiC-based heterojunctions have to be analyzed in future experiments in terms of possibly related capacitive properties.

However, based on the overall basic agreement regarding the numerical simulations of the absolute impedance characteristics as well as a quite good agreement of the corresponding phase characteristics in most parts of the spectrum, the basic parameters related to the capacitive elements of the system can be estimated by additional cross-sections of the characteristics at maximum applied bias voltages in forwardand reverse-bias direction of the diode. The resulting characteristics of absolute impedance $|Z(\omega)|$ (black) and corresponding phase $\theta(\omega)$ (blue) as a function of frequency at these constant voltages are illustrated in figure 8.12 .

All of them are in good agreement with the corresponding numerical simulations (red). However, considering the discrepancies at lower electric fields all over the frequency spectrum, the values of basic parameters related to the constant phase elements can only be roughly estimated based on these numerical simulations. Anyhow, the resulting values of 0.865 (ta-C) and 0.96 (diode) for the universal power $\nu$ and capacitances of $\widetilde{C}_{\mathrm{FP}} \approx 6 \mathrm{nF}(\mathrm{ta}-\mathrm{C})$ and $\widetilde{C}_{\mathrm{D}} \approx 1.3 \mathrm{nF}$ (diode) are comparable to ta-C/p-Si heterojunctions in section 8.2.1. 


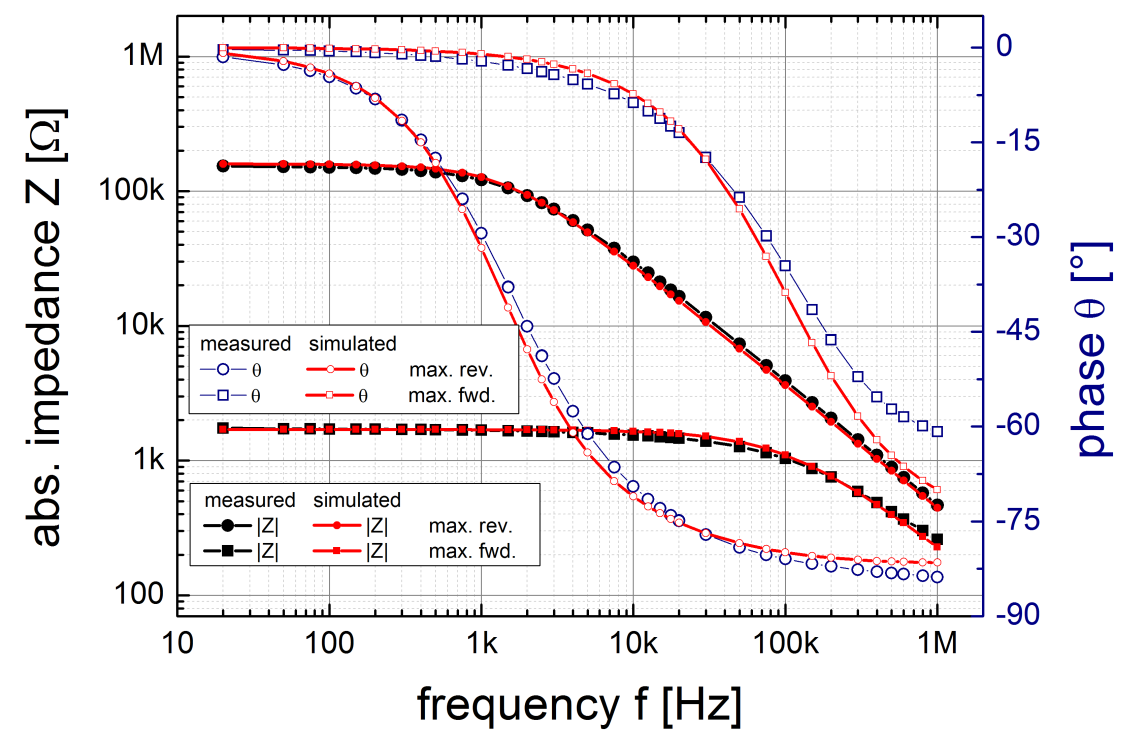

Figure 8.12: Absolute impedance $|Z(\omega)|$ (black) and corresponding phase $\theta(\omega)$ (blue) of a $\mathrm{Au} / \mathrm{Cr} / \mathrm{ta}-\mathrm{C} / \mathrm{SiC}$ heterojunction as a function of frequency at maximum applied bias voltages of $\pm 5 \mathrm{~V}$ in forward- (squares) and reverse-bias direction (circles) extracted from figures $8.11 \mathrm{~h}$ and b. 


\subsection{3 t-BN/SiC heterostructures}

Finally, the $\mathrm{AC}$ properties of $\mathrm{Au} / \mathrm{Cr} / \mathrm{t}-\mathrm{BN} / \mathrm{SiC}$ heterojunctions were investigated. The corresponding impedance characteristics $(|Z(V, \omega)|$ and $\theta(V, \omega)$, black circles) are shown in figure 8.13 including corresponding numerical simulations of the AC eFPID-model (red circles). The actual characteristics of the sample as well as the deviations of the corresponding numerical simulations are qualitatively very similar to ta-C based samples on $6 \mathrm{H}-\mathrm{SiC}$.

Therefore, despite a basic agreement of the numerical simulations with the actual absolute impedance characteristics as well as accordance of the corresponding phase characteristics, the AC eFPID-model still has to be revised in terms of voltage and frequency dependencies of the capacitive components regarding SiC-based heterojunction systems.

In addition, similar to ta-C/ $\mathrm{SiC}$ heterojunctions, these heterojunction also exhibit progressively increasing saturation processes regarding the forward-bias region at higher frequencies starting at above $2-3 \mathrm{kHz}$ and likely originated from the same, but yet unknown processes inside the grown thin-film structure.

Nevertheless, the basic parameters related to the capacitive elements of the system can again be estimated by additional cross-sections of the characteristics at maximum applied bias voltages in forward- and reverse-bias direction of the diode. The resulting characteristics of absolute impedance $|Z|$ (black) and corresponding phase $\theta$ (blue) as a function of frequency at these constant voltages are illustrated in figure 8.14. All of them are in good agreement with the corresponding numerical simulations (red). However, considering the discrepancies at lower electric fields all over the frequency spectrum, the values of basic parameters related to the constant phase elements can only be roughly estimated based on these numerical simulations. The resulting values of 0.94 (t-BN) and 0.96 (diode) for the universal power $\nu$ and capacitances of $\widetilde{C}_{\mathrm{FP}} \approx 1.2 \mathrm{nF}(\mathrm{t}-\mathrm{BN})$ and $\widetilde{C}_{\mathrm{D}} \approx 100 \mathrm{pF}$ (diode) are comparable to t-BN reference samples in section 8.2 .2 as well as ta-C/SiC based heterojunctions in section 8.3.2. 

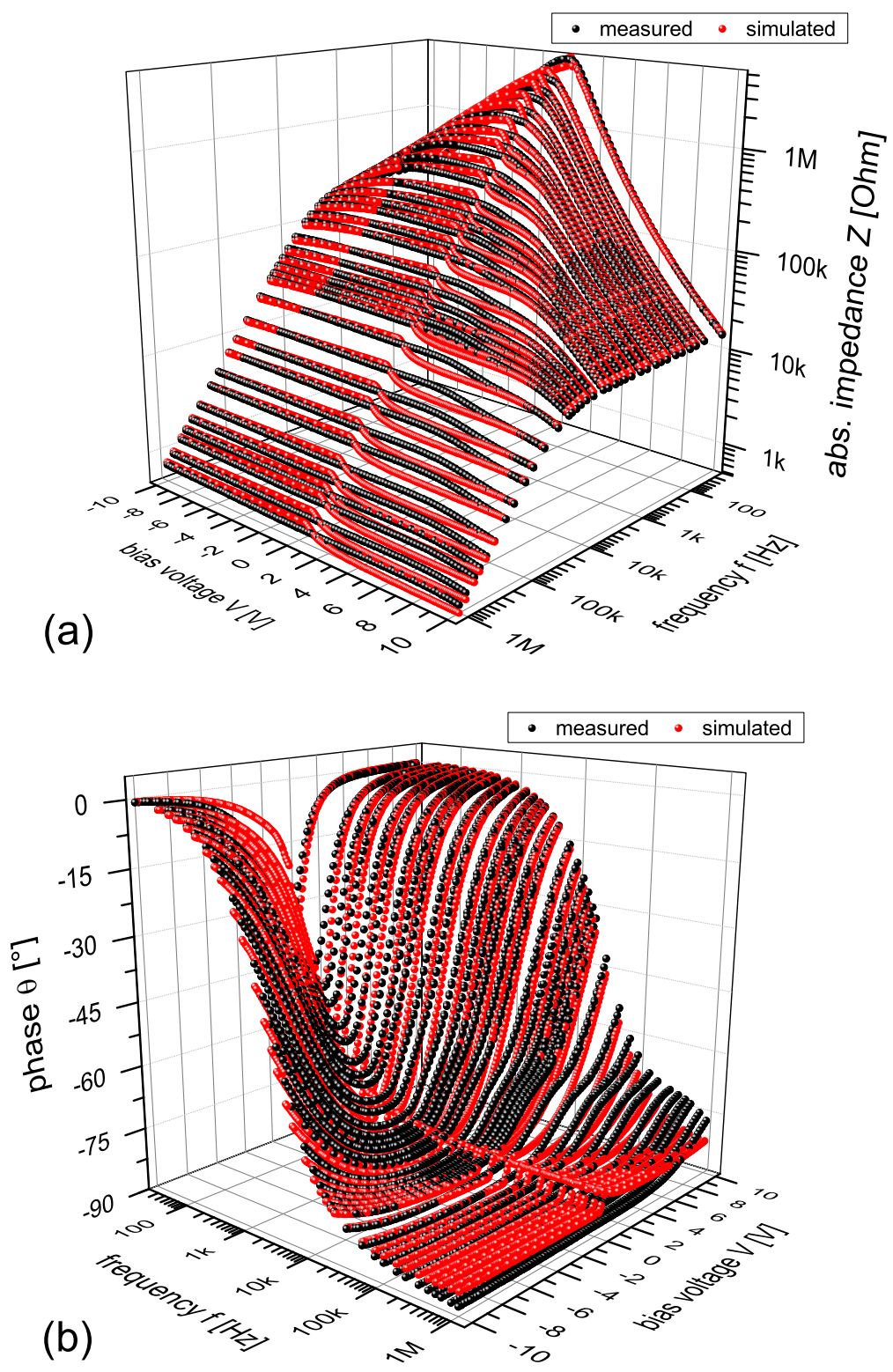

Figure 8.13: Typical complex impedance characteristic of a $\mathrm{Au} / \mathrm{Cr} / \mathrm{t}-\mathrm{BN} / \mathrm{SiC}$ heterojunction (black circles) including separate characteristics of the absolute impedance $|Z(V, \omega)|$ (a) and the corresponding phase $\theta(V, \omega)(\mathrm{b})$. The simulated datasets of the AC FP-model (red circles) according to figure $8.8 \mathrm{~b}$ are basically in good agreement with the recorded characteristics at high voltages but exhibit discrepancies at low- and mid-level bias voltages. 


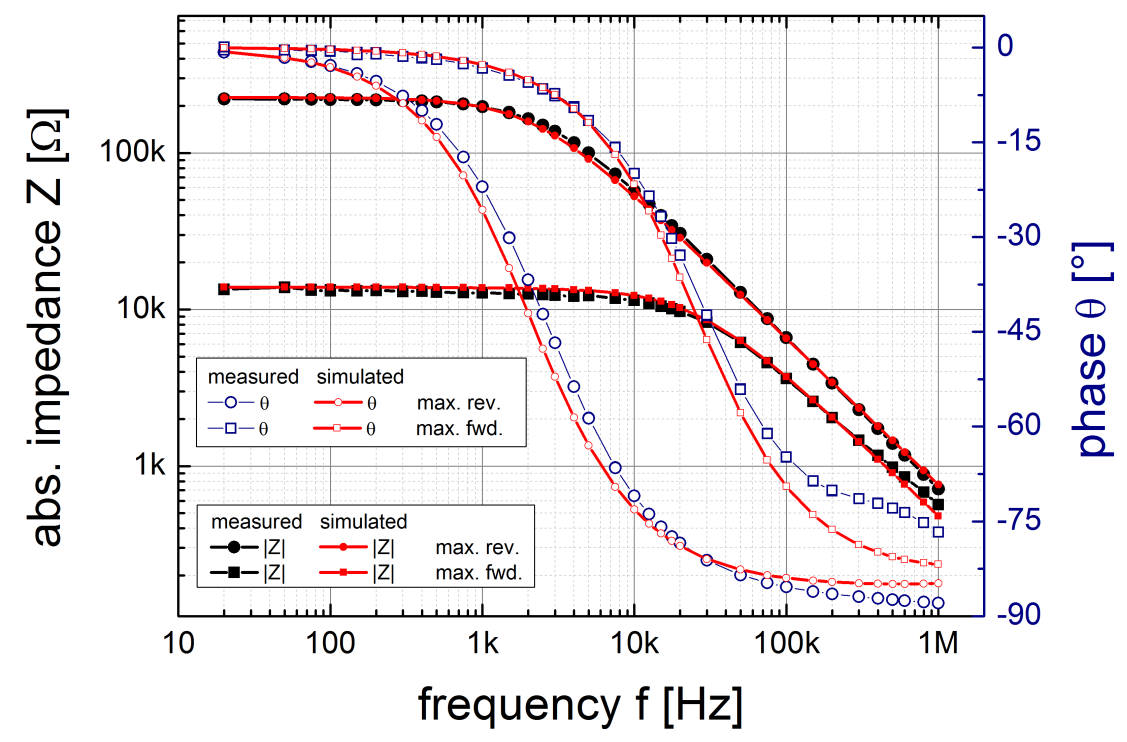

Figure 8.14: Absolute impedance $|Z(\omega)|$ (black) and corresponding phase $\theta(\omega)$ (blue) of a $\mathrm{Au} / \mathrm{Cr} / \mathrm{t}-\mathrm{BN} / \mathrm{SiC}$ heterojunction as a function of frequency at maximum applied bias voltages of $\pm 10 \mathrm{~V}$ in forward- (squares) and reverse-bias direction (circles) extracted from figures 8.13 and b. 


\subsection{Summary}

The general DC conduction models have been successfully extended with respect to the AC properties of the particular MASS diode systems resulting in basic overall agreement of the numerical simulations with the recorded impedance characteristics of the different MASS heterostructures.

The corresponding parameters of the resistors were solely adapted from the previous temperature dependent DC I-V measurement results in chapter 6] in order to guarantee consistent interpretation results of the recorded characteristics as no temperature dependent impedance measurement setup was available, yet. The parameters related to capacitive elements of the particular conduction models were primarily determined by plotting the absolute impedance as well as the phase as a function of frequency at several constant bias voltages. In this context, the voltages were selected on the basis of the corresponding DC fractional bias voltage characteristics of each heterojunction in chapter 6. Similar to the analysis of DC I-V characteristics, the basic parameters of the capacitive components related to the grown thin-films have been derived from impedance measurements of the corresponding reference samples.

While the numerical simulations are in good agreement with the recorded datasets at high electric fields, the absolute impedance is consistently overestimated around zero-bias in all investigated systems and accompanied by, to some extent, huge discrepancies regarding the corresponding phase characteristics.

In this context, corresponding voltage and frequency dependent saturation effects of the absolute impedance characteristics in several systems of this thesis indicate some kind of voltage dependencies of the related capacitive elements and are likely caused by the grown amorphous thin-films. Furthermore, the consistency of simulated and recorded datasets at higher frequencies based on t-BN reference samples implies that the observed discrepancies are merely restricted to the transition region between the dominating $\mathrm{DC}$ and $\mathrm{AC}$ conduction properties of the grown thin-films, at least for highly resistive thin-film materials such as t-BN.

Therefore, the extensions of the general DC conduction models based on the implemented constant phase elements just represent a raw approximation of the actual AC properties of the different MASS heterostructures and need to be analyzed in more detail in future experiments, especially with respect to the related voltage dependencies of the capacitive components and the corresponding structural properties of the particular heterostructure. 


\section{Chapter 9}

\section{Conclusion and Outlook}

In conclusion, a generalized temperature dependent conduction model has been successfully developed for all grown Metal-Amorphous Semiconductor-Semiconductor (MASS) heterojunctions diodes of this thesis.

The model is mainly based on the structural properties derived from the results of performed TEM-analyses of each particular heterostructure as well as the related electrical properties and corresponding temperature dependencies of the individual components forming the heterojunction. The corresponding equivalent circuit diagram of the extended Frenkel-Poole and ideal diode model (eFPID-model) is once again illustrated in figure 9.1.

The recorded datasets of all heterojunctions in this thesis can basically be well described by a serial arrangement of an ideal Schottky diode $\mathrm{D}(n=1)$, a voltage dependent resistor exhibiting Frenkel-Poole like conduction $R_{\mathrm{FP} 1}$ and an additional ohmic resistor $R_{\mathrm{S}}$ covering present contact and substrate resistances. Based on the structural properties of the interface region between crystalline semiconductor surface and the grown thin-films obtained from TEM-analyses, the leakage of the diode is approximated by a parasitic ohmic resistor $R_{\mathrm{P} 1}$ in order to represent the ohmic leakage currents inside the depletion region of the crystalline substrate and an additional Frenkel-Poole like resistor $R_{\mathrm{FP} 2}$ in case of a present amorphous interlayer interfering with the original heterojunction. As long as the interface does not exhibit any distinct amorphous interlayers, the two leakage resistors can be combined resulting in the simpler FPID-model. The resulting numerical simulations of this general conduction model are in very good agreement with the I-V characteristics of all investigated heterojunctions and were successfully applied to a total of eight different MASS-diode heterostructures.

Furthermore, the validity of the model as well as of the individual components within have been verified by additional temperature dependent measurements and corresponding numerical simulations. Deviations from the predicted characteristics below temperatures in the range of $150 \mathrm{~K}-200 \mathrm{~K}$, depending on the particular heterostructure compositions, are mainly caused by imperfections of the ohmic approximations regarding the I-V characteristics of leakage and serial resistors as well as the residual conductivity of the grown t-BN and ta-C thin-films. 


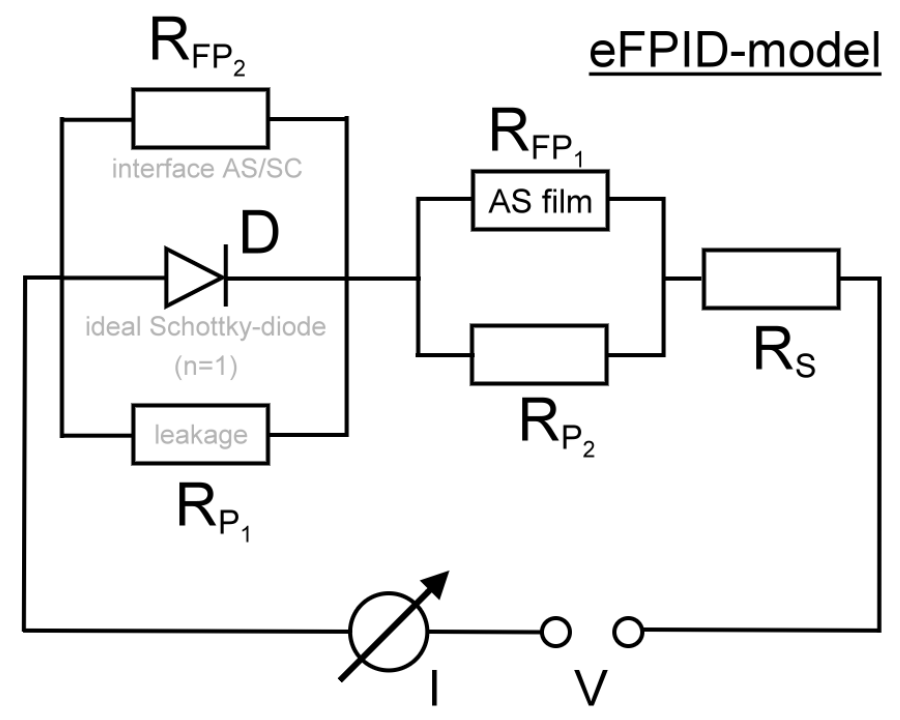

Figure 9.1: Equivalent circuit diagram of the extended Frenkel-Poole and ideal diode model (eFPID-model): A serial arrangement of an ideal Schottky diode $\mathrm{D}(n=1)$, a voltage dependent resistor exhibiting Frenkel-Poole like conduction $R_{\mathrm{FP} 1}$ and an additional ohmic resistor $R_{\mathrm{S}}$ covering present contact and substrate resistances. Based on the structural properties of the interface region between crystalline semiconductor surface and the grown thin-films obtained from TEM-analyses, the leakage of the diode is approximated by a parasitic ohmic resistor $R_{\mathrm{P} 1}$ in order to represent the ohmic leakage currents inside the depletion region of the crystalline substrate and an additional Frenkel-Poole like resistor $R_{\mathrm{FP} 2}$ in case of a present amorphous interlayer interfering with the original heterojunction. The resulting numerical simulations of this general conduction model are in very good agreement with the I-V characteristics of all investigated heterojunctions and were successfully applied to a total of eight different MASS-diode heterostructures.

Therefore, the shift towards a more temperature independent conduction mechanism should be investigated in more detail in future experiments of these heterostructures. Nevertheless, the results of Ronning et al. [57, 60] and Hofsäss et al. [151] regarding the electrical properties of ta-C and t-BN reference samples have additionally been verified on the basis of a more uniform Frenkel-Poole model (FP-model), developed in this work, down to temperatures of $200 \mathrm{~K}$ (ta-C) and $240 \mathrm{~K}$ (t-BN). In this context, additional parasitic currents at lower electric fields and temperatures as well as a temperature dependence of the thin-film conductivity proportional to $\exp (1 / \mathrm{T})$ were considered.

Furthermore, all MASS-herojunctions exhibit unusually high turn-on voltages and apparently high ideality factors of 30-200 in terms of the Shockley theory similar to the heterojunction diodes described by other groups (see chapter 1). Therefore, these structures might also possess an interfacial disordered or amorphous layer with Frenkel-Poole conduction properties dominating at forward-bias of the corresponding diode systems.

In terms of the structural properties, all grown t-BN as well as ta-C thin-films of this thesis each exhibit almost identical, uniform structural properties regardless 
of the underlying substrate. Therefore, the growth mechanism of the thin-films does not depend on the orientation or composition of the substrate. On the other hand, $\mathrm{Si}$ and $\mathrm{SiC}$ based heterostructures also exhibit amorphous interlayers between the grown thin-films and the substrate surface which is formed during the sputtercleaning process prior to the deposition of the thin-films. The formation of such an amorphous layer can exclusively be linked to damage cascades caused by implanted ions during the sputter-cleaning process based on corresponding TRIM-calculations. In this context, the particular ion ranges as well as recoil effects are primarily based on the material properties of the single crystalline semiconducting substrates and the resulting TRIM-calculations are in good agreement with the TEM-analyses of the resulting depths of the damaged surface of the substrate forming the amorphous interlayer.

As a result, the effects of damage cascades prior to the actual deposition processes should be minimized in upcoming experiments by reducing the ion-energies during the sputter-cleaning process. However, the results have to be frequently examined using cross-section TEM-analyses as well as DC I-V measurements in order to avoid delamination of the grown thin-films due to contaminants on top of the surface of the semiconducting substrates or an insufficient smoothening resulting in mechanical and electric breakthroughs.

Furthermore, in the beginning of the deposition process, an intermixing layer is formed in each system due to implantation of deposited ions into the surface layer of the semiconducting material.

Due to Sputtering and recoil effects on a small scale $(\AA-n m)$, the mixing process might lead to an incorporation of corresponding substrate- or dopant-atoms into the grown thin-films. The distribution of such kind of atoms inside the grown thin-films would depend on the solubility inside the particular thin-film matrices and on the corresponding sputter-yields and recoil distributions of these atoms. Hence, either a gradual distribution or a segregation process towards the surface would be the most likely results due to the dynamic sputtering process during deposition of the thin-films.

As the electrical properties of the grown thin-films are likely affected by these kind of processes, further experiments should be focused on a detailed analysis of intermixing-layer as well as amorphous interlayer compositions. Based on the observation of this thesis, the concentration of dopant atoms inside the grown thin-films as well as the resulting conductivity will likely correlate with the doping concentration inside the original substrates. Therefore, a detailed analysis of these effects is important for future extension of the developed conduction models. Additional measurement techniques such as Electron microprobe might provide more accurate information about the composition of these layers.

In this context, the effects of unintentional $\mathrm{Cu}$-contaminants on the conduction mechanism of the grown thin-films was also briefly covered in this thesis. As $\mathrm{Cu}$ contaminants only lead to an increase in conductivity in the range of 30-40 in ta-C as well as in t-BN thin-films while the average barrier height is unaffected, the con- 
duction models of this work are still valid for small amount of $\mathrm{Cu}$-contaminants. In addition, all MASS-heterojunctions exhibit a distinct photo-sensitivity in reversebias while the ta-C and t-BN reference samples do not show any signs of photoresponse effects. The fast rise times of photo currents in MASS-diode heterojunctions in the low nanosecond range indicate an immediate drain of the generated charge carries in each system, especially by applied reverse-bias voltages. However, the photoresponse decreases significantly at lower voltages reaching a minimum at zerobias.

Based on the results of I-V measurements of this work (chapter 6), this effect is accompanied by an increase of fractional bias voltage percentage across the grown thinfilms at the expense of the diode components in all investigated MASS-heterojunctions, especially around zero-bias. In addition, based on the results of the $\mathrm{SiC}$ based heterojunctions, the photoresponse of the heterojunctions is almost unaffected by the composition of the grown thin-films. Therefore, the distinct voltage dependence of photo-currents in all investigated MASS-diode systems indicates an absorption of light inside the depletion-layer region of the particular heterojunctions and therefore the presence of a one-sided p-n junction diode.

Moreover, the obtained decay time constants depend almost entirely on the substrate of the particular heterostructures and do not correspond to the properties of the grown thin-films. In fact, all performed measurements of this thesis do not indicate any interaction of light with the grown thin-films.

As the photoresponse of MASS-diode was only investigated on the basis of just two constant wavelengths of $266 \mathrm{~nm}$ and $532 \mathrm{~nm}$, wavelength dependent photoresponse measurements in the range of $200 \mathrm{~nm}-1200 \mathrm{~nm}$ might provide additional information about the wavelength dependent photoresponse of the substrates as well as wavelength dependent photoresponse characteristics of the grown thin-films based on the sensitivity of the measurement setup.

Finally, the general DC conduction models have been successfully extended with respect to the AC properties of the particular MASS-diode systems resulting in basic overall agreement of the numerical simulations with the recorded impedance characteristics of the different MASS heterostructures.

While the numerical simulations are in good agreement with the recorded datasets at high electric fields, the absolute impedance is consistently overestimated around zero-bias in all investigated systems and accompanied by, to some extent, huge discrepancies regarding the corresponding phase characteristics.

In this context, corresponding voltage and frequency dependent saturation effects of the absolute impedance characteristics in several systems of this thesis indicate voltage dependencies of the related capacitive elements and are likely caused by the grown amorphous thin-films. Furthermore, the consistency of simulated and recorded datasets at higher frequencies based on t-BN reference samples implies that the observed discrepancies are merely restricted to the transition region between the dominating $\mathrm{DC}$ and $\mathrm{AC}$ conduction properties of the grown thin-films, at least for highly resistive thin-film materials such as t-BN. 
Therefore, the extensions of the general DC conduction models based on the implemented constant phase elements just represent a raw approximation of the actual $\mathrm{AC}$ properties of the different MASS-heterostructures and need to be analyzed in more detail in future experiments, especially with respect to the related voltage dependencies of the capacitive components and the corresponding structural properties of the particular heterostructure.

In addition, based on the complexity of the investigated heterojunction diode systems of up to seven interfering components around zero-bias, the construction of a temperature dependent measurement setup is mandatory in order to separate the different resistors and reactances as well as the related temperature dependent parameters of the components. Moreover, such a setup might also provide valuable inside into the effect of residual conductivity of the grown thin-films due to potentially conclusive changes with respect to the capacitive behavior.

Finally, an upgrade of the auto-balance bridge, in terms of applicable frequencies, might improve the interpretation of the MASS-diode systems as the cut-off frequency of ta-C reference samples is close to the current frequency threshold. 


\section{List of Figures}

2.1 Schematic of a MASS heterostructure . . . . . . . . . 5

2.2 Cubic diamond structure of the silicon lattice . . . . . . . . . 6

2.3 Crystalline structures of $3 \mathrm{C}-, 4 \mathrm{H}-$ and $6 \mathrm{H}-\mathrm{SiC}$ polytypes . . . . . 8

2.4 Wurtzite structure of $\mathrm{ZnO} \ldots \ldots \ldots \ldots$

2.5 Typical 2" ZnO single crystal grown by a hydrothermal method . . . 11

2.6 Current-voltage characteristics of Al/p-type Si contacts . . . . . . . 13

2.7 Shadow mask of Si-substrates . . . . . . . . . . . . . . . 14

2.8 Shadow mask of SiC-substrates . . . . . . . . . . . . . . . . 15

2.9 Ternary phase-diagram of bonding in amorphous carbon - hydrogen

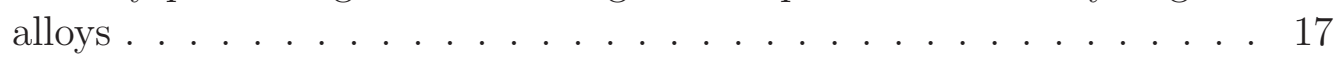

2.10 Microstructure of tetrahedral-amorphous carbon (ta-C) . . . . . . . 18

2.11 Crystalline structures of boron nitride . . . . . . . . . . . . . . 19

2.12 Properties of the t-BN structure . . . . . . . . . . . . . 20

2.13 In plan HRTEM image of a t-BN thin-film . . . . . . . . . . . . . 20

2.14 Setup of a Sidenius ion source . . . . . . . . . . . . . . . . . . . 21

2.15 Ion-accelerator ADONIS . . . . . . . . . . . . . . . . . 23

2.16 Typical shadow mask used for the evaporation of $\mathrm{Cr} / \mathrm{Au}$ top-contacts of the MASS structures . . . . . . . . . . . . . . . . . 25

3.1 Energy diagram of an ohmic contact at a metal-semiconductor or metal-insulator interface . . . . . . . . . . . . . 28 28

3.2 Schematic comparison of the electronic band structure of crystalline and amorphous dielectrics . . . . . . . . . . . . . . . . . . . . . . . . . .

3.3 The Frenkel-Poole emission process . . . . . . . . . . . . . . . 31

3.4 Simulated current-voltage characteristic of a Frenkel-Poole resistor . . 32

3.5 Schottky effect at an interfacial barrier . . . . . . . . . . . . 33

3.6 Simulated current-voltage characteristic of a Schottky resistor . . . . 33

3.7 Band-scheme of a pn-diode based on a semiconductor homojunction in a theoretical, decoupled state . . . . . . . . . . . . . 34

3.8 Band-scheme of a pn-diode based on a semiconductor homojunction in a coupled state . . . . . . . . . . . . . . . . 34

3.9 Formation of a space-charge region at thermal equilibrium conditions 35

3.10 Band-scheme of a biased pn-diode based on a semiconductor homojunction 
3.11 Band-scheme of a Schottky diode based on a metal/n-type semiconductor junction

3.12 Tunneling principle in a Schottky diode

3.13 Principle of photoconductivity

4.1 Principle of X-ray photoemission spectroscopy (XPS) . . . . . . . . 44

4.2 Typical SEM-images of cross-section TEM-lamella . . . . . . . . . . . 45

4.3 Probe geometries . . . . . . . . . . . . . . . . . . . 48

4.4 Sample holders . . . . . . . . . . . . . . . . . . . . . . 49

4.5 Electrical characterization setup (DC) . . . . . . . . . . . 50

4.6 Low-temperature setup . . . . . . . . . . . . . . . . . . 51

4.7 High-temperature setup . . . . . . . . . . . . . . 52

4.8 Basic layout of the data-processing setup . . . . . . . . . . . . . . . . . . . . . . . . 53

4.9 Impedance spectroscopy setup . . . . . . . . . . . . . . . 56

4.10 Photoconductivity setup of case study measurements . . . . . . . . 5 57

4.11 Cathodo-luminescence spectrum of a white LED used for case study photo-response measurements . . . . . . . . . . . . . 58

4.12 Time-resolved photoresponse setup . . . . . . . . . . . . . 59

5.1 Typical XPS-Spectrum of an Au-reference sample. . . . . . . . . . . 64

5.2 Typical XPS-spectrum of a typical ta-C thin-film . . . . . . . . . 66

5.3 Typical XPS-spectrum of a typical t-BN thin-film . . . . . . . . . 67

5.4 Determination of stoichiometry of a typical t-BN thin-film . . . . . 68

$5.5 \mathrm{Cu}$ contaminant analysis . . . . . . . . . . . . . . 69

5.6 SEM-image of a typical cross-section TEM-lamellas . . . . . . . . . . 71

5.7 TEM-images of a t-BN/ZnO heterostructure . . . . . . . . . 76

5.8 FFTs of TEM-images related to a t-BN/ZnO heterostructure . . . . . 77

5.9 TEM-images of a t-BN/n-Si heterostructure . . . . . . . . . . . 80

5.10 FFTs of TEM-images related to a t-BN/n-Si heterostructure . . . . . 81

5.11 TEM-images of a ta-C/n-Si heterostructure . . . . . . . . . . 83

5.12 FFTs of TEM-images related to a ta-C/n-Si heterostructure . . . . . 84

5.13 TEM-images of a t-BN/p-Si heterostructure . . . . . . . . . . 87

5.14 FFTs of TEM-images related to a t-BN/p-Si heterostructure . . . . . 88

5.15 TEM-images of a ta-C/p-Si heterostructure . . . . . . . . . . . . 90

5.16 FFTs of TEM-images related to a ta-C/p-Si heterostructure . . . . . 91

5.17 FFTs of crystalline clusters inside the interface region of a ta-C/p-Si heterostructure . . . . . . . . . . . . . . 92

5.18 Investigation of different interface structures in the ta-C/p-Si system 93

5.19 EDX-line-scans of a ta-C/p-Si heterostructure . . . . . . . . . . 94

5.20 EDX-line-scans of a $\mathrm{VO}_{2} / \mathrm{Si}$ heterostructure containing a $\mathrm{SiO}_{2}$-layer . 95

5.21 HRTEM-measurements of t-BN/SiC heterostructures . . . . . . . . . 98

5.22 FFTs of TEM-images related to a t-BN/SiC heterostructure . . . . . 99

$5.23 \mathrm{FFT}$ of TEM-images related to a t-BN/SiC heterostructure . . . . . 100

5.24 HRTEM-measurements of a ta-C/SiC heterostructure . . . . . . . . 102 
5.25 FFTs of TEM-images related to a ta-C/SiC heterostructure . . . . 103

5.26 Typical overall EDX-spectrum of a ta-C/SiC heterostructure . . . . . 104

5.27 EDX-line-scan of a ta-C/SiC heterostructure . . . . . . . . . . . . . . . . . . . 105

5.28 SEM-images of different SiC-surfaces . . . . . . . . . . . . . . . 108

5.29 Low magnification cross-section TEM-measurement of a typical heterostructure of the ta-C/SiC system . . . . . . . . . . . . . . 109

$6.1 \mathrm{I}-\mathrm{V}$ characteristic of an $\mathrm{Au} / \mathrm{Cr} / \mathrm{n}-\mathrm{Si} / \mathrm{Ag}$ interface structure . . . . . . 117

6.2 Temperature dependent I-V measurements of a typical t-BN reference sample . . . . . . . . . . . . . . . . . . . . . 118

6.3 Equivalent circuit diagram of the Frenkel-Poole model (FP-model) . . 119

6.4 Arrhenius-plots of a typical t-BN reference sample . . . . . . . . . . 122

6.5 Temperature dependent Schottky-plot of a typical ta-C reference sample 123

6.6 Arrhenius-plots of a typical ta-C reference sample. . . . . . . . . . . . 126

6.7 Comparison of I-V characteristics (Schottky plots) of typical t-BN and ta-C thin-films on metallic silicon with contaminated samples at room temperature . . . . . . . . . . . . . . . . . 127

6.8 Arrhenius-plot of a ta-C reference sample damaged by a partial electrical breakthrough . . . . . . . . . . . . . . . . . . 129

$6.9 \mathrm{I}-\mathrm{V}$ characteristic of a typical $\mathrm{Au} / \mathrm{Cr} / \mathrm{t}-\mathrm{BN} / \mathrm{ZnO}$ heterojunction at room temperature . . . . . . . . . . . . . . . . . 130

6.10 Frenkel-Poole and ideal diode model (FPID-model) . . . . . . . . . 131

$6.11 \mathrm{I}-\mathrm{V}$ characteristics of different back-contact materials on zinc oxide substrates . . . . . . . . . . . . . . . . . 133

6.12 Fractional bias voltages of a typical $\mathrm{Au} / \mathrm{Cr} / \mathrm{t}-\mathrm{BN} / \mathrm{ZnO}$ heterojunction at room temperature . . . . . . . . . . . . . . . . . 134

6.13 Non-ideal diode model (NID-model) . . . . . . . . . . . . . . . . 135

6.14 Temperature dependent I-V measurements of a typical $\mathrm{Au} / \mathrm{Cr} / \mathrm{t}-\mathrm{BN} / \mathrm{ZnO}$ heterojunction . . . . . . . . . . . . . . . . . 137

6.15 Fractional bias voltages of a typical $\mathrm{Au} / \mathrm{Cr} / \mathrm{t}-\mathrm{BN} / \mathrm{ZnO}$ heterojunction at different temperatures . . . . . . . . . . . . . . . . . . 138

6.16 Arrhenius-plot regarding the conductivity $\sigma_{0}$ of the t-BN thin-films inside $\mathrm{t}-\mathrm{BN} / \mathrm{ZnO}$ heterostructures . . . . . . . . . . . . . . . . 139

6.17 I-V characteristic of a typical $\mathrm{Au} / \mathrm{Cr} / \mathrm{ta}-\mathrm{C} / \mathrm{p}-\mathrm{Si}$ heterostructure at $300 \mathrm{~K} \ldots \ldots \ldots \ldots \ldots 14 \ldots \ldots \ldots \ldots$

$6.18 \mathrm{I}-\mathrm{V}$ characteristics of different back-contact materials on p-type silicon substrates . . . . . . . . . . . . . . . . . 143

$6.19 \mathrm{I}-\mathrm{V}$ measurement of a typical $\mathrm{Au} / \mathrm{Cr} / \mathrm{ta}-\mathrm{C} / \mathrm{p}-\mathrm{Si}$ heterojunction at high electric fields . . . . . . . . . . . . . . . . . . 145

6.20 Temperature dependent I-V measurements of a typical $\mathrm{Au} / \mathrm{Cr} / \mathrm{ta}-$ $\mathrm{C} / \mathrm{p}$-Si heterojunction . . . . . . . . . . . . . . . . 147

6.21 Arrhenius-plot regarding the conductivity $\sigma_{0}$ of the ta- $\mathrm{C}$ thin-films inside ta-C/p-Si heterostructures 
$6.22 \mathrm{I}-\mathrm{V}$ characteristics of $\mathrm{Al}$ and $\mathrm{Au} / \mathrm{Cr}$ top-contacts on ta-C/p-Si heterostructures

6.23 I-V measurement of a typical $\mathrm{Al} / \mathrm{ta}-\mathrm{C} / \mathrm{n}-\mathrm{Si}$ heterojunction at room temperature

6.24 Fractional bias voltages of an $\mathrm{Al} / \mathrm{ta}-\mathrm{C} / \mathrm{n}-\mathrm{Si}$ heterojunction at room temperature . . . . . . . . . . . . . . . . . . . . 152

6.25 I-V characteristics of different back-contact materials on $6 \mathrm{H}$ silicon carbide substrates . . . . . . . . . . . . . . . . . . 153

$6.26 \mathrm{I}-\mathrm{V}$ characteristic of a typical $\mathrm{Au} / \mathrm{Cr} / \mathrm{t}-\mathrm{BN} / \mathrm{SiC}$ heterojunction at $300 \mathrm{~K} 155$

6.27 Extended Frenkel-Poole and ideal diode model (eFPID-model) . . . . 156

6.28 Temperature dependent I-V measurement of a typical $\mathrm{Au} / \mathrm{Cr} / \mathrm{t}-\mathrm{BN} / \mathrm{SiC}$ heterojunction . . . . . . . . . . . . . . . . . . 158

6.29 Arrhenius-plot regarding the conductivity $\sigma_{0}$ of the t-BN thin-films inside $\mathrm{t}-\mathrm{BN} / \mathrm{SiC}$ heterostructures . . . . . . . . . . . . . . 159

$6.30 \mathrm{I}-\mathrm{V}$ characteristic of a typical $\mathrm{Au} / \mathrm{Cr} / \mathrm{ta}-\mathrm{C} / \mathrm{SiC}$ heterojunction at room temperature . . . . . . . . . . . . . . . . . . . 161

6.31 Temperature dependent I-V measurements of a typical $\mathrm{Au} / \mathrm{Cr} / \mathrm{ta}-$ $\mathrm{C} / \mathrm{SiC}$ heterojunction . . . . . . . . . . . . . . . . . . 164

6.32 Arrhenius-plot regarding the conductivity $\sigma_{0}$ of the ta- $\mathrm{C}$ thin-films inside ta-C/SiC heterostructures . . . . . . . . . . . . . . 165

$6.33 \mathrm{I}-\mathrm{V}$ characteristic of a typical $\mathrm{Au} / \mathrm{Cr} / \mathrm{t}-\mathrm{BN} / \mathrm{SiC}$ heterojunction containing Cu-contaminants . . . . . . . . . . . . . 167

6.34 I-V measurement of a typical $\mathrm{Au} / \mathrm{Cr} / \mathrm{t}-\mathrm{BN} / \mathrm{p}-\mathrm{Si}$ heterostructure at room temperature . . . . . . . . . . . . . . . . . . . 169

$6.35 \mathrm{I}-\mathrm{V}$ characteristic of a typical $\mathrm{Au} / \mathrm{Cr} / \mathrm{ta}-\mathrm{C} / \mathrm{ZnO}$ heterostructure at room temperature

6.36 Extended Frenkel-Poole and ideal diode model (eFPID-model) . . . . 174

7.1 Case study photoconductivity measurements of typical t-BN and ta-C reference samples . . . . . . . . . . . . . . . . . 178

7.2 Case study photoconductivity measurements of typical MASS diodes exemplarily shown on the basis of the t-BN/ZnO as well as the ta$\mathrm{C} / \mathrm{SiC}$ system . . . . . . . . . . . . . . . . . . . 181

7.3 Photoconductivity measurements of a ta-C/p-Si heterojunction. . . . 182

7.4 Time resolved photoresponse measurements of a typical $\mathrm{Au} / \mathrm{Cr} / \mathrm{ta}-$ $\mathrm{C} / \mathrm{p}$-Si heterojunction . . . . . . . . . . . . . . . . 185

7.5 Time resolved photoresponse measurements of a typical $\mathrm{Au} / \mathrm{Cr} / \mathrm{t}-$ $\mathrm{BN} / \mathrm{SiC}$ heterojunction . . . . . . . . . . . . . . . . . 187

7.6 Time resolved photoresponse measurements of a typical $\mathrm{Au} / \mathrm{Cr} / \mathrm{ta}-$ C/SiC heterojunction. . . . . . . . . . . . . . . . . . . . . . 189

7.7 Time resolved photoresponse measurements of a typical $\mathrm{Au} / \mathrm{Cr} / \mathrm{t}-$ $\mathrm{BN} / \mathrm{ZnO}$ heterojunction . . . . . . . . . . . . . . . . . . 191

8.1 Exemplary impedance circuit . . . . . . . . . . . . . . 195 
8.2 Absolute impedance and phase spectra of an exemplary impedance circuit . . . . . . . . . . . . . . . . . . 195

8.3 Typical complex impedance measurement of a ta-C reference sample . 199

8.4 Equivalent circuit diagram for AC impedance characteristics of amorphous thin-film reference samples . . . . . . . . . . . . . . . 200

8.5 Absolute impedance $|Z(\omega)|$ (black) and corresponding phase $\theta(\omega)$ (blue) of a typical ta-C reference sample at constant voltages . . . . . 201

8.6 Typical complex impedance measurement of a t-BN reference sample 202

8.7 Absolute impedance and corresponding phase of a typical t-BN reference sample at constant voltages . . . . . . . . . . . . . . . 203

8.8 Equivalent circuit diagrams of the AC-FPID- as well as the ACeFPID-model . . . . . . . . . . . . . . . . . . . 204

8.9 Typical complex impedance characteristic of a $\mathrm{Au} / \mathrm{Cr} / \mathrm{ta}-\mathrm{C} / \mathrm{p}-\mathrm{Si}$ heterojunction . . . . . . . . . . . . . . . . . . . . 206

8.10 Absolute impedance and corresponding phase of a $\mathrm{Au} / \mathrm{Cr} / \mathrm{ta}-\mathrm{C} / \mathrm{p}-\mathrm{Si}$ heterojunction at maximum applied bias voltages . . . . . . . . . 207

8.11 Typical complex impedance characteristic of a $\mathrm{Au} / \mathrm{Cr} / \mathrm{ta}-\mathrm{C} / \mathrm{SiC}$ heterojunction . . . . . . . . . . . . . . . . . . . . 208

8.12 Absolute impedance and corresponding phase of a $\mathrm{Au} / \mathrm{Cr} / \mathrm{ta}-\mathrm{C} / \mathrm{SiC}$ heterojunction at maximum applied bias voltages . . . . . . . . . . . 210

8.13 Typical complex impedance characteristic of a $\mathrm{Au} / \mathrm{Cr} / \mathrm{t}-\mathrm{BN} / \mathrm{SiC}$ heterojunction . . . . . . . . . . . . . . . . . . . . 212

8.14 Absolute impedance and corresponding phase of a $\mathrm{Au} / \mathrm{Cr} / \mathrm{t}-\mathrm{BN} / \mathrm{SiC}$ heterojunction at maximum applied bias voltages . . . . . . . . . 213

9.1 Extended Frenkel-Poole and ideal diode model (eFPID-model) . . . . 216 


\section{Bibliography}

[1] W. Shockley. The theory of pn junctions in semiconductors and pn junction transistors. Bell Syst. Tech. J, pages 435-489, 1949.

[2] J. Bardeen and W. H. Brattain. The Transistor, a Semiconductor Triode. Phys. Rev., 71:230, 1948.

[3] W. Shockley. Electrons and holes in semiconductors with applications to transistor electronics. Van Nostrand, 1950.

[4] J.S. Kilby. Invention of the integrated circuit. IEEE Transactions on Electron Devices, 23(7):648-654, July 1976.

[5] S. M. Sze and M. K. Lee. Semiconductor Devices - Physics and Technology. Wiley, 2012.

[6] Yoshitaka Taniyasu, Makoto Kasu, and Toshiki Makimoto. An aluminium nitride light-emitting diode with a wavelength of 210 nanometres. Nature, 441(7091):325-328, May 2006.

[7] C. R. Miskys, J. A. Garrido, M. Hermann, M. Eickhoff, C. E. Nebel, M. Stutzmann, and G. Vogg. Structural and interface properties of an AlN diamond ultraviolet light emitting diode. Appl. Phys. Lett., 85(17):3699-3701, October 2004.

[8] Cheng-Xin Wang, Guo-Wei Yang, Hong-Wu Liu, Yong-Hao Han, Ji-Feng Luo, Chun-Xiao Gao, and Guang-Tian Zou. Experimental analysis and theoretical model for anomalously high ideality factors in $\mathrm{ZnO} /$ diamond $\mathrm{p}-\mathrm{n}$ junction diode. Appl. Phys. Lett., 84(13):2427-2429, March 2004.

[9] D. M. Chapin, C. S. Fuller, and G. L. Pearson. A New Silicon p-n Junction Photocell for Converting Solar Radiation into Electrical Power. J. Appl. Phys, 25:676, 1954.

[10] H. Morkoc, S. Strite, G. B. Gao, M. E. Lin, B. Sverdlov, and M. Burns. Large-band-gap SiC, III-V nitride, and II-VI ZnSe-based semiconductor device technologies. J. Appl. Phys., 76(3):1363-1398, August 1994. 
[11] S. M. Sze and K. Ng. Kwok. Physics of Semiconductor Devices. Wiley, 3rd edition, 2006.

[12] M. Bhatnagar and B.J. Baliga. Comparison of 6H-SiC, 3C-SiC, and Si for power devices. Electron Devices, IEEE Transactions on, 40(3):645-655, 1993.

[13] A. Itoh and H. Matsunami. Analysis of Schottky Barrier Heights of Metal/SiC Contacts and Its Possible Application to High-Voltage Rectifying Devices. phys. stat. sol. (a), 162(1):389-408, 1997.

[14] Liang-Yu Chen, Gary W. Hunter, Philip G. Neudeck, Gaurav Bansal, Jeremy B. Petit, and Dak Knight. Comparison of interfacial and electronic properties of annealed $\mathrm{Pd} / \mathrm{SiC}$ and $\mathrm{Pd} / \mathrm{SiO}_{2} / \mathrm{SiC}$ Schottky diode sensors. In $J$. Vac. Sci. Technol. A, volume 15, pages 1228-1234, Philadelphia, Pennsylvania (USA), May 1997. AVS.

[15] K.P. Schoen, J.M. Woodall, J.A. Cooper, and M.R. Melloch. Design considerations and experimental analysis of high-voltage SiC Schottky barrier rectifiers. Electron Devices, IEEE Transactions on DOI - 10.1109/16.701494, 45(7):1595-1604, 1998.

[16] F. La Via, F. Roccaforte, A. Makhtari, V. Raineri, P. Musumeci, and L. Calcagno. Structural and electrical characterisation of titanium and nickel silicide contacts on silicon carbide. Microelectronic Engineering, 60(1-2):269282, January 2002.

[17] G. de Cesare, F. Irrera, F. Lemmi, and F. Palma. Tunable photodetectors based on amorphous $\mathrm{Si} / \mathrm{SiC}$ heterostructures. Electron Devices, IEEE Transactions on DOI - 10.1109/16.3819'r7, 42(5):835-840, 1995.

[18] P.G. Neudeck and J.A. Powell. Performance limiting micropipe defects in silicon carbide wafers. Electron Device Letters, IEEE, 15(2):63-65, 1994.

[19] J.B. Casady and R.W. Johnson. Status of silicon carbide (SiC) as a widebandgap semiconductor for high-temperature applications: A review. SolidState Electronics, 39(10):1409-1422, October 1996.

[20] D. Hofmann, R. Eckstein, M. Kölbl, Y. Makarov, St.G. Müller, E. Schmitt, A. Winnacker, R. Rupp, R. Stein, and J. Völkl. SiC-bulk growth by physicalvapor transport and its global modelling. Journal of Crystal Growth, 174(14):669-674, April 1997.

[21] S.G. Müller, R. Eckstein, D. Hofmann, E. Schmitt, W. Schoierer, A. Winnacker, W. Dorsch, and H.P. Strunk. Micropipes and polytypism as a source of lateral inhomogeneities in $\mathrm{SiC}$ substrates. Materials Science and Engineering: B, 44(1-3):392-394, February 1997. 
[22] Jennifer Giocondi, Gregory S. Rohrer, Marek Skowronski, V. Balakrishna, G. Augustine, H.M. Hobgood, and R.H. Hopkins. An atomic force microscopy study of super-dislocation/micropipe complexes on the 6H-SiC (0001) growth surface. Journal of Crystal Growth, 181(4):351-362, November 1997.

[23] J. Heindl, W. Dorsch, H. P. Strunk, St. G. Müller, R. Eckstein, D. Hofmann, and A. Winnacker. Dislocation Content of Micropipes in SiC. Phys. Rev. Lett., 80(4):740-741, January 1998.

[24] St.G. Müller, R.C. Glass, H.M. Hobgood, V.F. Tsvetkov, M. Brady, D. Henshall, J.R Jenny, D. Malta, and C.H. Carter Jr. The status of SiC bulk growth from an industrial point of view. Journal of Crystal Growth, 211(1-4):325-332, April 2000.

[25] St.G. Müller, R.C. Glass, H.M. Hobgood, V.F. Tsvetkov, M. Brady, D. Henshall, D. Malta, R. Singh, J. Palmour, and C.H. Carter Jr. Progress in the industrial production of $\mathrm{SiC}$ substrates for semiconductor devices. Materials Science and Engineering: B, 80(1-3):327-331, March 2001.

[26] St. G. Müller, J. J. Sumakeris, M. F. Brady, R. C. Glass, H. McD. Hobgood, J. R. Jenny, R. Leonard, D. P. Malta, M. J. Paisley, A. R. Powell, V. F. Tsvetkov, S. T. Allen, M. K. Das, J. W. Palmour, and C. H. Carter. Defects in $\mathrm{SiC}$ substrates and epitaxial layers affecting semiconductor device performance. The European Physical Journal - Applied Physics, 27(1-3):29-35, 2004.

[27] M. Tajima, E. Higashi, T. Hayashi, H. Kinoshita, and H. Shiomi. Nondestructive characterization of dislocations and micropipes in high-resistivity 6H-SiC wafers by deep-level photoluminescence mapping. Appl. Phys. Lett., 86(6):061914-3, February 2005.

[28] C.T. Sah, R.N. Noyce, and W. Shockley. Carrier generation and recombination in pn junctions and pn junction characteristics. Proceedings of the IRE, page $1228,1957$.

[29] S. K. Cheung and N. W. Cheung. Extraction of Schottky diode parameters from forward current-voltage characteristics. Appl. Phys. Lett., 49(2):85-87, July 1986.

[30] O. Pakma, N. Serin, T. Serin, and S. Altindal. The double Gaussian distribution of barrier heights in $\mathrm{Al} / \mathrm{TiO}_{2} / \mathrm{p}$-Si (metal-insulator-semiconductor) structures at low temperatures. J. Appl. Phys., 104(1):014501-6, July 2008.

[31] E. H. Rhoderick and R.H. Williams. Metal-Semiconductor Contacts. Clarendon, Oxford, 2nd edition, 1988.

[32] S.M. Sze. Physics of Semiconductor Devices. Wiley, 1981. 
[33] D. K. Schroeder. Semiconductor Material and Device Characterization. Wiley, New York, 1990.

[34] S. Mridha, R. Ghosh, and D. Basak. Bias-Dependent Ultraviolet Photodetection by $\mathrm{Au}-\mathrm{Mg}_{0.1} \mathrm{Zn}_{0.9} \mathrm{O} / \mathrm{ZnO}-\mathrm{Ag}$ Structure. Journal of Electronic Materials, 36(4):524-528, April 2007.

[35] Cheng-Xin Wang, Guo-Wei Yang, Tie-Chen Zhang, Hong-Wu Liu, Yong-Hao Han, Ji-Feng Luo, Chun-Xiao Gao, and Guang-Tian Zou. High-quality heterojunction between p-type diamond single-crystal film and n-type cubic boron nitride bulk single crystal. Appl. Phys. Lett., 83(23):4854-4856, December 2003.

[36] Hiromichi Ohta, Masahiro Hirano, Ken Nakahara, Hideaki Maruta, Tetsuhiro Tanabe, Masao Kamiya, Toshio Kamiya, and Hideo Hosono. Fabrication and photoresponse of a pn-heterojunction diode composed of transparent oxide semiconductors, p-NiO and n-ZnO. Appl. Phys. Lett., 83(5):1029-1031, August 2003.

[37] O. Breitenstein, P. Altermatt, K. Ramspeck, and A. Schenk. The origin if ideality factors $n>2$ of shunts and surfaces in the dark I-V curves of Si solar cells. 2006.

[38] Jay M. Shah, Y.-L. Li, Th. Gessmann, and E. F. Schubert. Experimental analysis and theoretical model for anomalously high ideality factors $(n \gg 2.0)$ in AlGaN/GaN p-n junction diodes. J. Appl. Phys., 94(4):2627-2630, August 2003.

[39] Abhishek Motayed, Ashok Sharma, Kenneth A. Jones, Michael A. Derenge, Agis A. Iliadis, and S. Noor Mohammad. Electrical characteristics of $\mathrm{Al}_{x} \mathrm{Ga}_{1-x} \mathrm{~N}$ Schottky diodes prepared by a two-step surface treatment. $J$. Appl. Phys., 96(6):3286-3295, September 2004.

[40] Abhishek Motayed, Albert V. Davydov, Mark D. Vaudin, Igor Levin, John Melngailis, and S. N. Mohammad. Fabrication of GaN-based nanoscale device structures utilizing focused ion beam induced Pt deposition. J. Appl. Phys., 100(2):024306-8, July 2006.

[41] A. Yoshida, H. Tamura, K. Gotoh, H. Takauchi, and S. Hasuo. Electrical properties of $\mathrm{Au} /$ and $\mathrm{YBa}_{2} \mathrm{Cu}_{3} \mathrm{O}_{7-x} / \mathrm{SrTi}_{1-y} \mathrm{Nb}_{y} \mathrm{O}_{3}$ diodes. J. Appl. Phys., 70:4976, 1991.

[42] Dae-Kue Hwang, Min-Suk Oh, Jae-Hong Lim, Yong-Seok Choi, and Seong-Ju Park. ZnO-based light-emitting metal-insulator-semiconductor diodes. Appl. Phys. Lett., 91(12):121113-3, September 2007. 
[43] Dae-Kue Hwang, Min-Suk Oh, Jae-Hong Lim, and Seong-Ju Park. ZnO thin films and light-emitting diodes. Journal of Physics D: Applied Physics, 40(22):R387-, 2007.

[44] P. Chen, X. Ma, and D. Yang. Appl. Phys. Lett., 89:111112, 2006.

[45] Andreas Schenk and Ulrich Krumbein. Coupled defect-level recombination: Theory and application to anomalous diode characteristics. J. Appl. Phys., 78(5):3185-3192, September 1995.

[46] H.C. Card. Photovoltaic properties of MIS-Schottky barriers. Solid-State Electronics, 20(12):971-976, December 1977.

[47] S. Ashok, J.M. Borrego, and R.J. Gutmann. Electrical characteristics of GaAs MIS Schottky diodes. Solid-State Electronics, 22(7):621-631, July 1979.

[48] S. Chand and J. Kumar. Semicond. Sci. Technol., 10:1680, 1995.

[49] Subhash Chand and Jitendra Kumar. Effects of barrier height distribution on the behavior of a Schottky diode. J. Appl. Phys., 82(10):5005-5010, November 1997.

[50] Ö. F. Yüksel, A. B. Selcuk, and S. B. Ocak. Physica B, 403:2690, 2008.

[51] H. C. Card and E. H. Rhoderick. J. Phys. D: Appl. Phys., 4:1589, 1971.

[52] J. H. Werner and H. H. Güttler. Barrier inhomogeneities at Schottky contacts. J. Appl. Phys., 69:1522, 1991.

[53] M. Brötzmann, U. Vetter, and H. Hofsäss. BN/ZnO heterojunction diodes with apparently giant ideality factors. J. Appl. Phys., 106(6):063704-9, September 2009.

[54] V. S. Veerasamy, G. A. J. Amaratunga, J. S. Park, W. I. Milne, H. S. MacKenzie, and D. R. McKenzie. Photoresponse characteristics of n-type tetrahedral amorphous carbon/p-type Si heterojunction diodes. Appl. Phys. Lett., 64(17):2297-2299, April 1994.

[55] Th. Mandel, M. Frischholz, R. Helbig, S. Birkle, and A. Hammerschmidt. Electrical and optical properties of heterostructures made from diamond-like carbon layers on crystalline silicon. Applied Surface Science, 65-66:795-799, March 1993.

[56] N. Konofaos and C. B. Thomas. Characterization of heterojunction devices constructed by amorphous diamondlike films on silicon. J. Appl. Phys., 81(9):6238-6245, May 1997. 
[57] C. Ronning, U. Griesmeier, M. Gross, H.C. Hofsäss, R.G. Downing, and G.P. Lamaze. Conduction processes in boron- and nitrogen-doped diamond-like carbon films prepared by mass-separated ion beam deposition. Diamond and Related Materials, 4(5-6):666-672, May 1995.

[58] H. Hofsäss. Electronic properties of undoped and doped tetrahedral amorphous carbon. page 296. World Scientific, Singapore, 1998.

[59] J. Frenkel. On Pre-Breakdown Phenomena in Insulators and Electronic SemiConductors. Phys. Rev., 54(8):647, October 1938.

[60] C. Ronning, E. Dreher, H. Feldermann, M. Gross, M. Sebastian, and H. Hofsäss. Electrical properties and thermal stability of ion beam deposited BN thin films. Diamond and Related Materials, 6(9):1129-1134, July 1997.

[61] K. Nose, H.S. Yang, and T. Yoshida. Electrical characterization of p-type cubic boron nitride/n-type silicon heterojunction diodes. Diamond and Related Materials, 14(8):1297-1301, August 2005.

[62] K. Nose, H.S. Yang, H. Oba, and T. Yoshida. Defect-induced electronic conduction of tBN thin films. Diamond and Related Materials, 14(11-12):19601963, 2005.

[63] K. Nose, H. Oba, and T. Yoshida. Electric conductivity of boron nitride thin films enhanced by in situ doping of zinc. Appl. Phys. Lett., 89(11):112124-3, September 2006.

[64] C. Kittel. Einführung in die Festkörperphysik. Oldenbourg, 2006.

[65] J. K. Rath. Low temperature polycrystalline silicon: a review on deposition, physical properties and solar cell applications. Solar energy materials and solar cells, 76(4):431, 2003.

[66] David J. Lockwood and Lorenzo Pavesi. Silicon Fundamentals for Photonic Application, volume 94 of Topics in Applied Physics. Springer, 2004.

[67] B. G. Gribov and K. V. Zinov'ev. Preparation of High-Purity Silicon for Solar Cells. Inorganic Materials, 39(7):653-662, 2003.

[68] Landolt-Börnstein. Vol.41: Semiconductors Group III Condensed Matter, Group IV Elements, IV-IV and III-V Compounds. Part A - Lattice Properties. Springer, 2001.

[69] Martin A. Green. Intrinsic concentration, effective densities of states, and effective mass in silicon. J. Appl. Phys., 67(6):2944-2954, March 1990.

[70] H.D. Barber. Effective mass and intrinsic concentration in silicon. Solid-State Electronics, 10(11):1039-1051, November 1967. 
[71] J. Czochralski. Ein neues Verfahren zur Messung der Kristallisationsgeschwindigheit der Metalle Zeitschrift für physikalische Chemie. Zeitschrift für physikalische Chemie, 92:219-221, 1918.

[72] G. L. Harris. Properties of Silicon Carbide. INSPEC, The Institution of Electrical Engineers, 1995.

[73] Yu. Goldberg, M.E. Levinshtein, and S.L. Rumyantsev. Properties of Advanced SemiconductorMaterials GaN, AlN, SiC, BN, SiC, SiGe. John Wiley \& Sons, Inc., New York, 2001.

[74] Ulrich Vetter. Lanthanide Doped Wide Band Gap Semiconductors: Intra-4f Luminescence and Lattice Location Studies. PhD thesis, University of Göttingen, 2003.

[75] Yu.M. Tairov and V.F. Tsvetkov. General principles of growing large-size single crystals of various silicon carbide polytypes. Journal of Crystal Growth, 52, Part 1(0):146-150, April 1981.

[76] M. Ikeda, H. Matsunami, and T. Tanaka. Site effect on the impurity levels in 4H, 6H, and 15R SiC. Phys. Rev. B, 22(6):2842-2854, September 1980.

[77] A. Lebedev. Deep level centers in silicon carbide: A review. Semiconductors, 33(2):107-130, 1999.

[78] A.A. Kalnin, V.V. Pasynkov, Y.M. Tairoy, and D.A. Yaskov. Investigation of P-junctions Produced on the Basis of Silicon Carbide Alloyed with Beryllium. DTIC Document, 1967.

[79] C. H. Park, Byoung-Ho Cheong, Keun-Ho Lee, and K. J. Chang. Structural and electronic properties of cubic, $2 \mathrm{H}, 4 \mathrm{H}$, and $6 \mathrm{H} \mathrm{SiC.} \mathrm{Phys.} \mathrm{Rev.} \mathrm{B,}$ 49(7):4485-4493, February 1994.

[80] N. T. Son, O. Kordina, A. O. Konstantinov, W. M. Chen, E. Sorman, B. Monemar, and E. Janzen. Electron effective masses and mobilities in high-purity 6H-SiC chemical vapor deposition layers. Appl. Phys. Lett., 65(25):3209-3211, December 1994.

[81] U. Ozgur, Ya. I. Alivov, C. Liu, A. Teke, M. A. Reshchikov, S. Dogan, V. Avrutin, S.-J. Cho, and H. Morkoc. A comprehensive review of $\mathrm{ZnO}$ materials and devices. J. Appl. Phys., 98(4):041301-103, August 2005.

[82] S.J. Pearton, D.P. Norton, K. Ip, Y.W. Heo, and T. Steiner. Recent progress in processing and properties of ZnO. Progress in Materials Science, 50(3):293340, March 2005.

[83] J. E. Jaffe and A. C. Hess. Hartree-Fock study of phase changes in ZnO at high pressure. Phys. Rev. B, 48(11):7903-7909, September 1993. 
[84] Hiroyuki Kato, Michihiro Sano, Kazuhiro Miyamoto, and Takafumi Yao. Growth and characterization of Ga-doped ZnO layers on a-plane sapphire substrates grown by molecular beam epitaxy. Journal of Crystal Growth, 237-239, Part 1(0):538-543, April 2002.

[85] Akihiko Sandhu Adarsh Takahashi, Kiyoshi; Yoshikawa. Wide bandgap semiconductors: fundamental properties and modern photonic and electronic devices. Springer, 2007.

[86] H.C. Card. Aluminum-Silicon Schottky barriers and ohmic contacts in integrated circuits. Electron Devices, IEEE Transactions on, 23(6):538-544, 1976.

[87] J. Basterfield, J.M. Shannon, and A. Gill. The nature of barrier height variations in alloyed Al-Si Schottky barrier diodes. Solid-State Electronics, 18(3):290-, March 1975.

[88] J. Crofton, P. G. McMullin, J. R. Williams, and M. J. Bozack. Hightemperature ohmic contact to n-type 6H-SiC using nickel. J. Appl. Phys., 77(3):1317-1319, February 1995.

[89] J. Crofton, L. M. Porter, and J. R. Williams. The Physics of Ohmic Contacts to $\mathrm{SiC}$. phys. stat. sol. (b), 202(1):581-603, 1997.

[90] Han-Ki Kim, Sang-Heon Han, Tae-Yeon Seong, and Won-Kook Choi. Lowresistance $\mathrm{Ti} / \mathrm{Au}$ ohmic contacts to Al-doped ZnO layers. Appl. Phys. Lett., 77(11):1647-1649, September 2000.

[91] K. Ip, G.T. Thaler, Hyucksoo Yang, Sang Youn Han, Yuanjie Li, D.P. Norton, S.J. Pearton, Soowhan Jang, and F. Ren. Contacts to ZnO. Journal of Crystal Growth, 287(1):149-156, January 2006.

[92] M. Backhaus-Ricoult. Metal-Ceramic Interfaces. Acta-Scripta Metall. Proc. Pergamon Press, New York, 1990.

[93] T. Uemoto. Reduction of Ohmic Contact Resistance on n-Type 6H-SiC by Heavy Doping. Japanese Journal of Applied Physics, 34, 1995.

[94] A. K. Chaddha, J. D. Parsons, and G. B. Kruaval. Thermally stable, low specific resistance $\left(1.30 \times 10^{-5} \Omega \mathrm{cm}^{2}\right)$ TiC Ohmic contacts to n-type $6 \mathrm{H} \alpha$ SiC. Appl. Phys. Lett., 66(6):760-762, February 1995.

[95] H. Yang, T.H. Peng, W.J. Wang, D.F. Zhang, and X.L. Chen. Ta/Ni/Ta multilayered ohmic contacts on n-type SiC. Applied Surface Science, 254(2):527531, November 2007.

[96] B. Pecz. Contact formation in SiC devices. Applied Surface Science, 184(14):287-294, December 2001. 
[97] S. Liu, K. Reinhardt, C. Severt, and J. Scofield, 1996.

[98] G. Kelner, S. Binari, M. Shur, and J. Palmour. High temperature operation of $\alpha$-silicon carbide buried-gate junction field-effect transistors. Electronics Letters, 27(12):1038 - 1040, 1991.

[99] Ts. Marinova, A. Kakanakova-Georgieva, V. Krastev, R. Kakanakov, M. Neshev, L. Kassamakova, O. Noblanc, C. Arnodo, S. Cassette, C. Brylinski, B. Pecz, G. Radnoczi, and Gy. Vincze. Nickel based ohmic contacts on SiC. Materials Science and Engineering B, 46(1-3):223-226, April 1997.

[100] H. Sheng, N. Emanetoglu, S. Muthukumar, B. Yakshinskiy, S. Feng, and Y. Lu. Ta/Au ohmic contacts to n-type ZnO. Journal of Electronic Materials, 32(9):935-938, 2003-09-01.

[101] A Inumpudi, A.A Iliadis, S Krishnamoorthy, S Choopun, R.D Vispute, and T Venkatesan. P-Ga Ohmic contacts to n-ZnO using focused ion beams. SolidState Electronics, 46(10):1665-1668, October 2002.

[102] J. Robertson. Diamond-like amorphous carbon. Materials Science and Engineering: R: Reports, 37(4-6):129-281, May 2002.

[103] P.B. Mirkarimi, K.F. McCarty, and D.L. Medlin. Review of advances in cubic boron nitride film synthesis. Materials Science and Engineering: R: Reports, 21(2):47-100, December 1997.

[104] W. Niesser. PhD thesis, Universität Giessen, 2000.

[105] D. G. Mc Culloch, R. Mc Kenzie, and S. Prawer. Compressive stress induced formation of preferred orientation in glassy carbon following high-dose $\mathrm{C}^{+}$ implantation. Philosophical Magazine A, 72(4):1031-1041, October 1995.

[106] H. Hofsäss, C. Ronning, U. Griesmeier, M. Gross, S. Reinke, and M. Kuhr. Cubic boron nitride films grown by low energy $\mathrm{B}^{+}$and $\mathrm{N}^{+}$ion beam deposition. Appl. Phys. Lett., 67(1):46-48, July 1995.

[107] Sören Eyhusen. Phase formation processes in the synthesis of boron nitride thin films. PhD thesis, Universität Göttingen, 2005.

[108] K.F. McCarty, P.B. Mirkarimi, D.L. Medlin, T.A. Friedmann, and J.C. Barbour. On the low-temperature threshold for cubic boron nitride formation in energetic film deposition. Diamond and Related Materials, 5(12):1519-1526, December 1996.

[109] G. Sidenius. The high-temperature hollow cathode ion source. Nucl. Instr. and Meth .Phys. Res. B 38, page 19, 1965. 
[110] G. Sidenius. Ion sources for low energy accelerators. Nuclear Instruments and Methods, 151(3):349-362, May 1978.

[111] Inga Hannstein. Self-Organized Formation of Metal-Carbon Nanostructures by Hyperthermal Ion Deposition. PhD thesis, Universität Göttingen, 2006.

[112] H. Hofsäss, H. Feldermann, R. Merk, M. Sebastian, and C. Ronning. Cylindrical spike model for the formation of diamondlike thin films by ion deposition. Applied Physics A: Materials Science 63 Processing, 66(2):153-181, February 1998.

[113] A.K. Jonscher. Electronic properties of amorphous dielectric films. Thin Solid Films, 1(3):213-234, November 1967.

[114] J. G. Simmons. Conduction in thin dielectric films. Journal of Physics D: Applied Physics, 4(5):613-657, 1971.

[115] Paul Drude. Zur Elektronentheorie der Metalle. Annalen der Physik, 306(3):566-613, 1900.

[116] Robert A. Millikan and E. S. Bishop. Elements of Electricity. American Technical Society, 1917.

[117] N. Mott. Conduction in glasses containing transition metal ions. Journal of Non Crystalline Solids, 1:1-17, December 1968.

[118] E. A Mott, N. F. ; Davis. Electronic processes in non-crystalline materials. Clarendon Press Oxford, 1979.

[119] M Pollak and I Riess. A percolation treatment of high-field hopping transport. Journal of Physics C: Solid State Physics, 9:2339, 1976.

[120] Harald Ibach and Hans Lüth. Festkörperphysik - Einführung in die Grundlagen. Springer, 2002.

[121] http://wikipedia.org/.

[122] Franz Schwabl. Quantenmechanik. Springer-Verlag Berlin, 6 edition, 2005.

[123] J. J. O'Dwyer. The Theory of Electrical Conduction and Breakdown in Solid Dieelectrics. Clarendon Press Oxford, 1973.

[124] S. M. Sze. Current Transport and Maximum Dielectric Strength of Silicon Nitride Films. J. Appl. Phys., 38(7):2951-2956, June 1967.

[125] L. Sullivan and H. C. Card. Conduction in amorphous thin films of silicon nitride under non-uniform electric fields. Journal of Applied Physics, 7:1531, 1974. 
[126] J. J. O'Dwyer. Current-Voltage Characteristics of Dielectric Films. Journal of Applied Physics, 37(2):599, Feb 1966.

[127] L. A. DeWerd and P. R. Moran. Solid-state electrophotography with $\mathrm{Al}_{2} \mathrm{O}_{3}$. Med. Phys., 5(1):23-26, January 1978.

[128] M. Freitag, Y. Martin, J. A. Misewich, R. Martel, and Ph. Avouris. Photoconductivity of Single Carbon Nanotubes. Nano Letters, 3(8):1067-1071, August 2003.

[129] S. Bhushan and A. Oudhia. Photoconductivity and photoluminescence in chemically deposited films of $\mathrm{CdSSe}: \mathrm{CdCl}_{2}, \mathrm{Ho}$. Opto-Electronics Review, 17(1):30-39, March 2009.

[130] A. Einstein. Annalen der Physik, 19:132, 1905.

[131] H.R. Verma. Atomic and Nuclear Analytical Methods. Springer, Berlin Heidelberg, 2007.

[132] Hayo Zutz. Growth and Characterization of Carbon-Metal-NanocompositeThin-Films and Self-Organized Layer Growth. PhD thesis, University of Göttingen, 2009.

[133] http://www.python.org/download/releases/2.7.2/.

[134] http://wxpython.org/.

[135] J. A. Amani. Voltage-dependent impedance analysis of the metal/ta-C/Si heterostructure. Master's thesis, Georg-August Universität Göttingen, 2012.

[136] John C. Fuggle and Nils Må rtensson. Core-level binding energies in metals Journal of Electron Spectroscopy and Related Phenomena, 1980. Journal of Electron Spectroscopy and Related Phenomena, 21(3):275-281, 1980.

[137] P Merel, M Tabbal, M Chaker, S Moisa, and J Margot. Direct evaluation of the $\mathrm{sp}^{3}$ content in diamond-like-carbon films by XPS. Applied Surface Science, 136(1-2):105-110, October 1998.

[138] S. D. Berger, D. R. McKenzie, and P. J. Martin. EELS analysis of vacuum arcdeposited diamond-like films. Philosophical Magazine Letters, 57(6):285-290, June 1988.

[139] P. Widmayer, H.-G. Boyen, P. Ziemann, P. Reinke, and P. Oelhafen. Electron spectroscopy on boron nitride thin films: Comparison of near-surface to bulk electronic properties. Phys. Rev. B, 59(7):5233, February 1999.

[140] J. J. Yeh and I. Lindau. Atomic subshell photoionization cross sections and asymmetry parameters: $1 \leq Z \leq 103$. Atomic Data and Nuclear Data Tables, 32(1):1-155, January 1985. 
[141] Dominika Lyzwa. Synthese und Charakterisierung von selbstorganisierten aC:Cu-Multilagen. Master's thesis, Universität Göttingen, Februar 2008.

[142] David B. Williams and C. Barry Carter. Transmission Electron Microscopy A Textbook for Materials Science. Springer, 2009.

[143] J.F. Ziegler. SRIM2008. http://www.srim.org.

[144] M. Brötzmann. Wachstum und Charakterisierung von BN/ZnO- sowie BN/SiHeterostrukturen. Master's thesis, University of Göttingen, 2008.

[145] Amir H. Al-Bayati, Kevin G. Orrman-Rossiter, Ranjan Badheka, and D.G. Armour. Radiation damage in silicon (001) due to low energy (60-510 eV) argon ion bombardment. Surface Science, 237(1-3):213-231, November 1990.

[146] Paul Ehrhardt. Lokale Modifikation der elektrischen Eigenschaften von Vanadiumdioxid durch Spuren hochenergetischer Schwerionen. Master's thesis, Universität Göttingen, 2013.

[147] R Yakimova, M Syväjärvi, T Iakimov, H Jacobsson, R Råback, A Vehanen, and E Janzén. Polytype stability in seeded sublimation growth of $4 \mathrm{H}-\mathrm{SiC}$ boules. Journal of Crystal Growth, 217(3):255-262, August 2000.

[148] N.G. van der Berg, Johan B. Malherbe, A.J. Botha, and E. Friedland. Thermal etching of SiC. Applied Surface Science, 258(15):5561-5566, May 2012.

[149] Marc Brötzmann, Ulrich Vetter, and Hans Hofsäss. ta-C/Si heterojunction diodes with apparently giant ideality factors. physica status solidi (c), $7(2): 256-259,2010$.

[150] Marc Brötzmann, Hans-Gregor Gehrke, Ulrich Vetter, and Hans Hofsäss. Modeling the diode characteristics of boron nitride/silicon carbide heterojunctions. Appl. Phys. Lett., 97(10):103505-3, September 2010.

[151] H.C. Hofsäss, J. Biegel, C. Ronning, R.G. Downing, and G.P. Lamaze. Characterization of Doped Diamondlike Carbon Films and Multilayers. MRS Proceedings, 316:881, 1994.

[152] W. Fukarek. In situ characterization of thin film growth: Boron nitride on silicon. In J. Vac. Sci. Technol. A, volume 19, pages 2017-2024, Boston, Massachusetts (USA), July 2001. AVS.

[153] S.R.P. Silva, R.U.A. Khan, A.P. Burden, J.V. Anguita, J.M. Shannon, B.J. Sealy, A.J. Papworth, C.J. Kiely, and G.A.J. Amaratunga. The microstructural dependence of the opto-electronic properties of nitrogenated hydrogenated amorphous carbon thin films. Thin Solid Films, 332(1-2):118-123, November 1998. 
[154] A. A. Evtukh. Electronic conductivity of nitrogen-doped diamond-like carbon films: Poole-Frenkel and Fowler-Nordheim mechanisms. Ukr. J. Phys., 53(10):983-990, 2008.

[155] H.-G. Gehrke. Electrical characterization of conductive ion tracks in amorphous tetrahedral carbon with copper impurities. $\mathrm{PhD}$ thesis, University of Göttingen, 2013.

[156] C. R. Wronski, D. E. Carlson, and R. E. Daniel. Schottky-barrier characteristics of metal-amorphous-silicon diodes. Appl. Phys. Lett., 29(9):602-605, November 1976.

[157] A.K. Jonscher. Frequency-dependence of conductivity in hopping systems. Journal of Non-Crystalline Solids, 8-10(0):293-315, June 1972.

[158] A. K. Jonscher. The 'universal' dielectric response. Nature, 267(5613):673-679, June 1977.

[159] A.K. Jonscher. Dielectric relaxation in solids. Chelsea Dielectric, 1983.

[160] J. C. Dyre and T. B. Schroeder. Universality of ac conduction in disordered solids. Rev. Mod. Phys., 72:873-892, July 2000.

[161] H. Scher, Michael F. Shlesinger, and J. T. Bendler. Time-scale invariance in transport and relaxation. Physics Today, 44(1):26-34, 1991.

[162] M. P. J. van Staveren, H. B. Brom, and L. J. de Jongh. Metal-cluster compounds and universal features of the hopping conductivity of solids. Physics Reports, 208((1)):1-96, 1991.

[163] M. Abjowitz et al. Handbook of Electronic and Photonic Materials. Springer Science+Business Media, Inc., 2006.

[164] A. G. Hunt. AC hopping conduction: Perspective from percolation theory. Philosophical Magazine Part B, 81(9):875-913, 2001.

[165] E. H. Nicollian and J. R. Brews. MOS (Metal Oxide Semiconductor) Physics and Technology. John Wiley \& Sons, Dezember 2002. 


\section{Curriculum vitae}

Persönliche Daten:

$\begin{array}{ll}\text { Name: } & \text { Marc Brötzmann } \\ \text { Geburtstag: } & 18.01 .1983 \\ \text { Geburtsort: } & \text { Braunschweig } \\ \text { Nationalität: } & \text { deutsch }\end{array}$

Bildungsweg:

2002 Abitur an der Eckener Oberschule in Berlin

2002-2003 Zivildienst in Berlin

2003 Beginn Physik-Studium an der

Georg-August Universität Göttingen

$2006 \quad$ Vordiplom Physik

2007-2009 Diplomarbeit am 2. Physikalischen Institut, Universität Göttingen, bei Prof. Dr. Hofsäss

2009-2012 Doktorarbeit am 2. Physikalischen Institut

Universität Göttingen, bei Prof. Dr. Hofsäss 



\section{List of Publications}

\section{Publications contributing to this thesis}

- M. Brötzmann, U. Vetter, and H. Hofsäss: "BN/ZnO heterojunction diodes with apparently giant ideality factors", J. Appl. Phys., 106(6):063704-9, September 2009.

- M. Brötzmann, U. Vetter, and H. Hofsäss: "ta-C/Si heterojunction diodes with apparently giant ideality factors", physica status solidi (c), 7(2):256-259, 2010.

- M. Brötzmann, H.-G. Gehrke, U. Vetter, and H. Hofsäss: "Modeling the diode characteristics of boron nitride/silicon carbide heterojunctions", Appl. Phys. Lett., 97(10):103505-3, September 2010.

\section{Other Publications}

- U. Vetter, S. Müller, M. Brötzmann, H. Hofsäss, and John B. Gruber: "Effective reduction of AlN defect luminescence by fluorine implantation", Diamond and Related Materials 20 (2011) 782

- K. Zhang, M. Brötzmann, H.Hofsäss: "Surfactant-driven self-organized surface patterns by ion beam erosion", New Journal of Physics 13, 013033, 2011.

- H. Hofsäss, P. Ehrhardt, H.-G. Gehrke, M. Brötzmann, U. Vetter, K. Zhang, J. Krauser, C. Trautmann, C. Ko, S. Ramanathan: "Tuning the conductivity of vanadium dioxide films on silicon by swift heavy ion irradiation", AIP Advances 1, 032168, 2011.

- K. Zhang, M. Brötzmann, H. Hofsäss: "Sharp transition from ripple patterns to a flat surface for ion beam erosion of Si with simultaneous co-deposition of iron", AIP Advances 2, 032123, 2012.

- H. Hofsäss, K. Zhang, A. Pape, O. Bobes, and M. Brötzmann: "The role of phase separation for self-organized surface pattern formation by ion beam erosion and metal atom co-deposition", Applied Physics A, 1-12, 2012. 



\section{Danksagung}

Zunächst möchte ich mich bei Herrn Prof. Dr. Hofsäß dafür bedanken, dass er mir die Möglichkeit gegeben hat, meine Doktorarbeit in seiner Arbeitsgruppe anzufertigen. Zudem möchte ich mich an dieser Stelle auch noch einmal für die zahlreichen Dienstreisen nach Weimar, Prag und natürlich Puerto Rico bedanken.

Herrn Prof. Dr. Michael Seibt danke ich sehr für die Bereitschaft, diese Arbeit zu korreferieren sowie für die zahlreichen kleinen Hilfestellungen und die stets sehr angenehme Atmosphäre bei unseren Unterhaltungen über die letzten Jahre.

Darüber hinaus auch ein großes Dankeschön an Prof. Dr. Angela Rizzi, Prof. Dr Cynthia A. Volkert, Prof. Dr. Hans-Ulrich Krebs und PD Dr. Jörn Grosse-Knetter, die sich allesamt sehr spontan bereit erklärt haben, für meine Disputation als Prüfer zur Verfügung zu stehen.

Vielen Dank auch an Herrn Dr. Ulrich Vetter, der mir in den letzten Jahren stets mit Rat und Tat zur Seite gestanden hat und auch einen großen Anteil daran hat, dass ich die letzten Wochen und Monate durchgestanden habe.

Ein weiterer großer Dank an Henning Schuhmann für das spontane Korrekturlesen der Arbeit und die zahlreichen kleinen Hilfestellungen, wenn es am TEM mal nicht weiter ging.

Weiterhin möchte ich Tristan Koppe und Christoph Brüsewitz für die vielen kleinen Hilfestellungen im Labor sowie die regelmäßigen Kaffeerunden danken. Zudem ein Dank an Julian Amani, der durch den Aufbau der Kapazitätsmessung und die Entwicklung der Auswertungssoftware einen wichtigen Beitrag zu diesem Teil meiner Arbeit geliefert hat. Ein besonderer Dank ist zudem an die Werkstätten des 2.

Physikalischen Instituts für die zahlreiche Hilfe insbesondere bei den nicht enden wollenden Reparaturen von ADONIS. Stellvertretend für die Feinmechanik sei hier Herr Klaus Arndt genannt sowie Volker Ebrecht aus der Elektronikwerkstatt für die vielen Extraschichten nach Feierabend.

Besonders hervorheben möchte ich außerdem Peter Arnsberger und Andreas König: Es hat unglaublich viel Spaß gemacht, mit Euch zu arbeiten und vielen Dank fürs Zuhören.

Danke auch an Herrn Herwig Schultz und unsere Sekretärinnen Frau Herbold und 
Frau Tyson, die mit so mancher kleinen Hilfestellungen zum Gelingen dieser Arbeit beigetragen haben.

Einen großen Dank auch an Paul Ehrhardt für die vielen tollen Gespräche and auch für die Hilfe an langen Wochenenden im Labor-Schichtdienst sowie Andre Pape für die vielen lustigen Momente und auch die vielen Gespräche.

Zudem ein großer Dank an Daniel Jürgens, der mit seiner Hilfsbereitschaft und Freundschaft einen großen Anteil der Fertigstellung dieser Arbeit hat.

Ein ganz großer Dank an Hans-Gregor Gehrke, für sein Verständnis, seine Anteilnahme und für die Freundschaft, die uns verbindet. Danke für Alles!

Schließlich möchte ich mich von ganzem Herzen bei meinen Eltern und Jürgen bedanken, dass wir dieses schwierige Jahr zusammen durchgestanden haben und kann nur sagen, dass diese Arbeit ohne Euch und Eure fortwährende Unterstützung nicht zustande gekommen wäre. Zu guter letzt noch ein großer Dank an meinen Großvater, meine Großmutter, Bini, Wilfried und Inge für die tolle Unterstützung und Anteilnahme sowie an alle anderen, die mir stets die Daumen gedrückt und mich unterstützt haben. 